Departamento de Inteligencia Artificial

Escuela Técnica Superior de Ingenieros Informáticos

$\mathrm{PhD}$ Thesis

\title{
Semantically-enabled Browsing of Large Multilingual Document Collections
}

\author{
Author: $\quad$ Carlos Badenes-Olmedo \\ Supervisors: $\quad$ Prof. Dr. Oscar Corcho
}


Tribunal nombrado por el Sr. Rector Magfco. de la Universidad Politécnica de Madrid, el día 9 de Junio de 2021.
Presidente: Dr. Horacio Saggion
Vocal: $\quad$ Dr. Raphaël Troncy
Vocal: $\quad$ Dr. Jerónimo Arenas García
Vocal: $\quad$ Dr. Jose Manuel Gómez Pérez
Secretario: Dr. Victor Rodriguez Doncel
Suplente: Dra. Elena Montiel Ponsoda
Suplente: Dr. Mariano Fernandez López

Realizado el acto de defensa y lectura de la Tesis el día 22 de Junio de 2021 en la Escuela Técnica Superior de Ingenieros Informáticos

Calificación:

EL PRESIDENTE

VOCAL 1

VOCAL 2

VOCAL 3

EL SECRETARIO 
A mis padres.

A Beatriz.

A Martín y Alonso. 


\section{Acknowledgements}

Although I didn't know it yet, this journey began almost 10 years ago in Boston, with Susana Muñoz. A study about the Ivory Coast, a presentation at M.I.T. and a strong determination to continue learning. New plans and a new future. Oscar Corcho then appeared, and thanks to him everything started to take shape. I discovered science in the Ontology Engineering Group, with great researchers who are also great colleagues. Asunción Gómez, Jorge Gracia, Victor Fernandez, Raul Garcia, Elena Montiel, Mariano Fernandez, Jose Angel Ramos and Mariano Rico. Particularly impressive were the talks with María Poveda, Dani Vila, Julia Bosque and Daniel Garijo. New ideas and unforeseen approaches came up in every talk. I admire you. Especially grateful to your accurate comments, Daniel. Research means reading, understanding, testing and also collaborating. I am very grateful for the interactions with Filip Radulovic and Idafen Santana, with Pablo Calleja and Rafael Gonzalez, with Nandana Mihindukulasooriya and Freddy Priyatna, with Esteban Gonzalez and with Fernando Serena, and the dedicated support of Raul Alcazar and Miguel Angel, because you forced me to be more pragmatic. And I cannot forget the DiversiTeam, where all the extra skills are put into practice. I don't want to forget anyone, you are all part of this work: David Chaves, Paola Espinoza, Francisco Yedro, Alba Fernandez, Maria Navas, Patricia Martin, Elvira Amador, Edna Ruckhaus, Alvaro Brandon, Serge Chavez, Andres Prados, Ana Iglesias, and Julian Arenas.

Special mention for my supervisor, Oscar Corcho, and my mentor, Jose Luis Redondo. Both of you have the ability to choose the right words and simplify any task. You have complemented each other perfectly, even from the distance, and I have enjoyed every year of this thesis. Oscar knows 
how to touch the right keys at the right time, and Jose Luis reduces each problem to the challenge that motivates it, no matter how complex it was. I am extremely lucky to have you by my side on this journey, and and your influence will guide my future steps.

Thank you so much Beatriz for offering me so much help this time. These have been very intense years, with many changes and challenges, but we have been able to keep smiling and move forward. I hope Martin and Alonso feel proud of us for having achieved our objectives. I will miss our nights working with their crying in the background.

Federico and Loli, I had imagined it otherwise, but I know you will be proud of me. Not for the thesis, but for the decisions that led me to do it. You are in everything I do, and thanks to your effort this achievement has become a reality. I also have to thank the patience of my sisters, Ana and Lucia, who have always been able to push me forward. 


\begin{abstract}
Searching for similar documents and exploring the major themes are common activities when browsing document collections. With the ongoing growth in the number of digital documents in multiple languages, we need better tools to browse large multilingual corpora. Manual document annotation has been traditionally used to facilitate such document browsing. However, manual annotation is knowledge-intensive and tedious task and can be alleviated by using automatic document annotation algorithms. Most algorithms represent documents in a common feature space that abstracts them away from the specific sequence of words used in them. Probabilistic Topic Models reduce that feature space by annotating documents with thematic information. Over this low-dimensional latent space some algorithms have been proposed to perform document similarity search, including collections of texts in multiple languages. However, dictionaries or comparable corpora are required to create multilingual topics and thematic information is usually hidden behind specific representations that limits the explanatory capability of topics to justify content-based similarities. In this thesis we address the challenge of automatically relating documents from large multilingual corpora based on the knowledge offered by the topics covered in the collection, and without the need for theme-aligned data. In order to do so, we have created a framework where probabilistic topic models can be created and reused, a hierarchical model for describing documents with thematic annotations and an unsupervised algorithm that relates multilingual documents from their most relevant themes. Evaluations on classifying and sorting documents by similar content reveal good results on multiple domains.
\end{abstract}




\section{Resumen}

La búsqueda de documentos similares y la exploración de los principales temas tratados son actividades comunes cuando se examinan colecciones de documentos. Con el continuo crecimiento del número de documentos digitales en múltiples idiomas, se necesitan mejores herramientas que permitan la navegación de corpus multilingües de gran tamaño. Tradicionalmente se ha utilizado anotaciones manuales para facilitar esa exploración. Sin embargo, es una tarea tediosa que requiere conocimiento del dominio, y puede aliviarse mediante algoritmos automáticos de anotación de documentos. La mayoría de los algoritmos representan documentos en un espacio de características comunes que los abstraen de la secuencia específica de palabras utilizadas en ellos. Los modelos probabilísticos de tópicos reducen ese espacio de características anotando los documentos con información temática. Sobre este espacio latente de reducidas dimensiones se han propuesto algoritmos que realizan búsquedas de documentos semejantes, incluso en colecciones de textos en múltiples idiomas. Sin embargo, para crear temas multilingües se necesitan datos o diccionarios que permitan alinear los temas y la información temática queda oculta tras representaciones que limitan su capacidad explicativa para justificar las relaciones basadas en el contenido. En esta tesis abordamos el desafío de relacionar automáticamente documentos multilingües a gran escala sin perder el conocimiento que ofrecen los temas para explicar las relaciones y sin necesitar corpus paralelos o comparables. Para ello, hemos creado un marco de trabajo donde se pueden crear y reutilizar modelos probabilísticos de tópicos, un modelo jerárquico para describir documentos con anotaciones temáticas y un algoritmo no supervisado que relaciona documentos multilingües a partir de sus principales temas. Las evaluaciones exhaustivas en múltiples dominios han mostrado buenos resultados en tareas de clasificación y recuperación de documentos por contenido similar. 


\section{Contents}

List of Figures

List of Tables

$\begin{array}{lll}\text { Acronyms } & \text { Xxvii }\end{array}$

1 Introduction 1

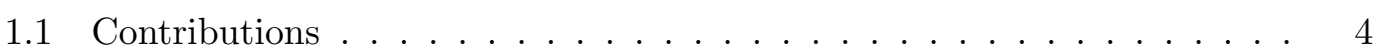

1.2 Thesis Structure . . . . . . . . . . . . . . . . 5

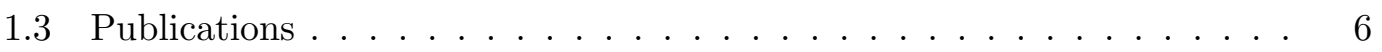

2 State of the Art 11

2.1 Information Retrieval . . . . . . . . . . . . . . . . 11

2.2 Techniques for Document Retrieval . . . . . . . . . . . . . . . 13

2.3 Techniques for Probabilistic Topic Models . . . . . . . . . . . . . . 20

2.3.1 Document Similarity Calculation based on LDA . . . . . . . 22

2.4 Research Areas in Topic Modelling Techniques . . . . . . . . . . . 2 26

2.4 .1 Topic Creation and Reuse . . . . . . . . . . . 27

2.4 .2 Topic Explainability . . . . . . . . . . . . . . . . . 2 29

2.4.3 Document Similarity . . . . . . . . . . . . . . . . . 30

2.4.4 Multilingual Topic Alignment . . . . . . . . . . . . . . . . 34

3 Methodology 39

3.1 Research Hypotheses . . . . . . . . . . . . . . . . . . . . . . 40

3.2 Research Challenges . . . . . . . . . . . . . . . . . 42 42

3.2.1 Scalable Creation and Inference of Topics . . . . . . . . . . . 42

3.2.2 Explainable Topic-based Relations . . . . . . . . . . . . . 43 
3.2.3 Large-scale Comparisons of Topic Distributions . . . . . . . . . 4 43

3.2.4 Unsupervised Cross-lingual Topic Alignment . . . . . . . . . . . 44

3.3 Research Methodology . . . . . . . . . . . . . . . . 44

4 Creation and Distribution of Probabilistic Topic Models 51

4.1 Topic Modeling Framework . . . . . . . . . . . . . . . . . . . . 51

4.1.1 Corpora Representation for Topic Modeling . . . . . . . . . . 53

4.1.1.1 Domain . . . . . . . . . . . . . . 5 55

4.1.1.2 Document ............... . . 55

4.1.1.3 Snippet .................. . . 57

4.1.1.4 Annotation . . . . . . . . . . . . . 57

4.1.2 Event-oriented Processing Workflow . . . . . . . . . . 58

4.1.3 Module-based Model Training . . . . . . . . . . . . . . . . . . 60 60

4.2 Topic Modeling Services . . . . . . . . . . . . . . . . . . . . 62

4.2.1 Topic Model Publication . . . . . . . . . . . . . . . . . 63

4.2 .2 Topic Model Exploitation . . . . . . . . . . . . . . . 63

4.2.2.1 Reproducibility Tasks . . . . . . . . . . . . 66 66

4.2.2.2 Exploration Tasks . . . . . . . . . . . . . . 67

4.2.2.3 Inference Tasks . . . . . . . . . . . . . . . . . . 68

4.3 Summary . . . . . . . . . . . . . . . . . . 68 68

5 Explainable Topic-based Associations 7

5.1 Topic-based Relations . . . . . . . . . . . . . . . . . . 72

$5.1 .1 \quad$ Research Articles Summaries . . . . . . . . . . . . . . 77

5.1 .2 Feature Vectors . . . . . . . . . . . . . . . . . 74

5.1 .3 Similarity Measure . . . . . . . . . . . . . . . . . 75

5.1 .4 Evaluation . . . . . . . . . . . . . . 75

5.1.4.1 Internal Representativeness . . . . . . . . . . . 76

5.1.4.2 External-Representativeness . . . . . . . . . 77

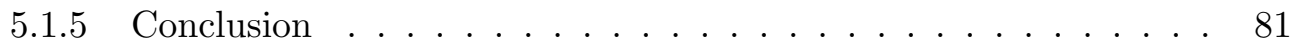

5.2 Topic-based Clustering . . . . . . . . . . . . . . . . . . 82

5.2 .1 Most Relevant Topics . . . . . . . . . . . . . . . . 82

5.2.1.1 Trends-based Clustering Method . . . . . . . . . . 84

5.2.1.2 Ranking-based Clustering Method . . . . . . . . . 85 
5.2.1.3 Cumulative Ranking-based Clustering Method . . . . . 85

5.2 .2 Evaluation . . . . . . . . . . . . . . 86

5.2 .2 .1 Datasets .................. 87

5.2 .2 .2 Settings . . . . . . . . . . . . . 87

5.2 .2 .3 Baselines . . . . . . . . . . . . . . 88

5.2 .2 .4 Measures . . . . . . . . . . . . . 89

5.2 .2 .5 Results . . . . . . . . . . . . . 90

5.2 .3 Conclusion . . . . . . . . . . . . . . . 97

5.3 Summary . . . . . . . . . . . . . . . . . . 99

6 Large-scale Monolingual Document Similarity 101

6.1 Hashing Topic Distributions . . . . . . . . . . . . . . . . . . . 101

6.1 .1 Hierarchical Data . . . . . . . . . . . . . . . . . . . . . 103

6.1 .2 Distance Metric . . . . . . . . . . . . . . . . . . . . . . 104

6.1 .3 Hash Function . . . . . . . . . . . . . . . . . 105

6.1.3.1 Threshold-based Hierarchical Hashing Method . . . . . 105

6.1.3.2 Centroid-based Hierarchical Hashing Method . . . . . . 105

6.1.3.3 Density-based Hierarchical Hashing Method . . . . . . 106

6.1.4 Online-mode Hashing ． . . . . . . . . . . . . . . . . . 107

6.2 Evaluation . . . . . . . . . . . . . . . . . . 107

6.2.1 Datasets and Evaluation Metrics . . . . . . . . . . . . . 108

6.2.2 Retrieving Related Documents . . . . . . . . . . . . . . . . 110

6.2 .3 Exploration . . . . . . . . . . . . . . . . . . . . 118

6.3 Summary . . . . . . . . . . . . . . . . . . . 119

7 Cross-lingual Document Similarity

7.1 Synset-based Representational Space . . . . . . . . . . . . . . . . 125

7.1 .1 Document representation . . . . . . . . . . . . . 126

7.1 .2 Similarity metric . . . . . . . . . . . . . . . . 126

7.1.3 Cross-lingual Models . . . . . . . . . . . . . . . . . . . 127

7.2 Evaluation . . . . . . . . . . . . . . . . . . . . 129

7.2.1 Cross-lingual Document Classification . . . . . . . . . . . 132

7.2.2 Cross-lingual Information Retrieval . . . . . . . . . . . . . . 133

7.2.3 Text Length on Cross-lingual Representations . . . . . . . . . . . 134 
7.3 Summary . . . . . . . . . . . . . . . . . 142

8 Real-World Use Cases

8.1 Scientific Creativity and Innovation . . . . . . . . . . . . . . 145

8.2 Innovation and Research in the ICT sector . . . . . . . . . . . . . . . 148

8.3 Polypharmacy and Drug-drug Interactions . . . . . . . . . . . . . . 149

8.4 Public Procurement Data . . . . . . . . . . . . . . . . . . 152

8.5 COVID-19 and Coronavirus-related Research . . . . . . . . . . 153

9 Conclusions and Future Work

9.1 Contributions . . . . . . . . . . . . . . . . . . . . . . 158

9.1.1 Efficient Creation and Use of Probabilistic Topic Models . . . . . 158

9.1.2 Explainability of Topic-based Relations among Documents . . . 160

9.1.3 Complexity Reduction in Comparisons among Topic Distributions at Large-Scale . . . . . . . . . . . . . . . . . 161

9.1.4 Multilinguality through Monolingual Topic Models . . . . . . . . 163

9.2 Impact . . . . . . . . . . . . . . . . . . . . . . . . . . 164

9.3 Future Directions . . . . . . . . . . . . . . . . . . . . . . . . . 168

9.3.1 Efficiency in Learning Models . . . . . . . . . . . . . . . . . . 168

9.3.2 Explainability of Text Similarity . . . . . . . . . . . . . 169

9.3.3 Complexity of Large-scale Comparisons . . . . . . . . . . . . . 171

9.3.4 Multilingual Representations . . . . . . . . . . . . . . . . . 172 


\section{List of Figures}

2.1 Documents listed in Section 1.3 described by word embeddings and projected in a two-dimensional space by PCA . . . . . . . . . . . . . . 18

2.2 Documents listed in Section 1.3 described by probabilistic topics (Table 2.2 and projected in a two-dimensional space by PCA. . . . . . . . 20

2.3 Evolution of the distances based on KL (a) and JS (b) metrics between a set of document pairs when increasing the number of topics in the models (Badenes-Olmedo et al. 2020b) . . . . . . . . . . . . . . . 31

2.4 Evolution of the distances based on He (a) and S2JSD (b) metrics between a set of document pairs when increasing the number of topics in the models (Badenes-Olmedo et al. 2020b) . . . . . . . . . . . . .

3.1 Research dimensions of the thesis. The first ones must be overcome before reaching higher dimensions. . . . . . . . . . . . . . . . .

4.1 Stages in the creation and reuse of topic models. Texts are first processed to retrieve tokens and create bags-of-words (BoW). These structures are used to train a model that identifies word distributions called topics. The model is enabled to make topic inferences in unseen texts. It is published as a web service in an online repository. The service can then be (re)used as web resource, for example to categorize documents. . . .

4.2 Representation of two scientific papers published at the International Conference on Knowledge Capture (K-CAP, 2019) that mention the same entity, Wordnet, in different sections. . . . . . . . . . . . . 554

4.3 Relation between domain and document. . . . . . . . . . . . . 55

4.4 Relation between document and snippet. . . . . . . . . . . . . 57 
4.5 Relation between annotations and other resources. . . . . . . . . 58

4.6 Resource states. . . . . . . . . . . . . . . . . . . . . 559

4.7 Modules (in gray) publishing or receiving events from the messenger service (in purple) . . . . . . . . . . . . . . . . . . . 60

4.8 Sequence of events exchanged between modules to create a topic model from the documents added to a domain. . . . . . . . . . . . . . 62

4.9 OpenAPI-based web interface of a probabilistic topic model service created with librAIry. . . . . . . . . . . . . . . . . . 65

5.1 Internal and External Representativeness. . . . . . . . . . . . 73

5.2 Experiment to analyze the ability of topic-based representations to create relations from summaries vs full-texts. . . . . . . . . . . . . . . 74

5.3 length of summaries. . . . . . . . . . . . . . . 76

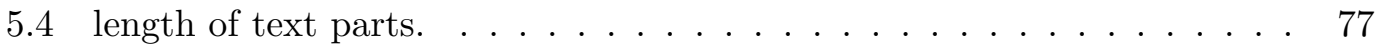

5.5 number of pairwises by similarity score (rounded up to two decimals). . 78

5.6 topics per article with value above $0.5 \ldots \ldots \ldots$. . . . . . . . . . .

5.7 Precision at different similarity thresholds. . . . . . . . . . . . . . . 80

5.8 Recall at different similarity thresholds. . . . . . . . . . . . . . . 80

5.9 f-measure performance. . . . . . . . . . . . . . . . 81

5.10 f-measure deviation. . . . . . . . . . . . . . . . . 81

5.11 Probabilistic Topic Models, and in particular Latent Dirichlet Allocation (LDA), can efficiently divide the search space and speed up the process of finding relations among documents inside big collections. . . . . . . . 83

5.12 TDC considers variations across consecutive topics inside a document's topic distribution. . . . . . . . . . . . . . . . 84

$5.13 \mathrm{RDC}$ only considers the top $\mathrm{n}$ topics from the ranked list of probability distributions. . . . . . . . . . . . . . . 85

5.14 CRDC only considers the top n topics until the sum of the weights of the highest topics exceeded a given threshold. . . . . . . . . . 86

5.15 Similarity values grouped by frequency in AIES . . . . . . . . . . 88

5.16 Effectiveness (JS-based) in AIES . . . . . . . . . . . . . . . . . 91

5.17 Effectiveness (He-based) in AIES . . . . . . . . . . . . . . . 91

5.18 Clusters in AIES . . . . . . . . . . . . . . . . . . . . 92 
5.19 Cost (JS-based) in AIES . . . . . . . . . . . . . . . . . 94

5.20 Cost (He-based) in AIES . . . . . . . . . . . . . . . 95

5.21 Efficiency $($ JS-based) in AIES . . . . . . . . . . . . . . 959

5.22 Efficiency (He-based) in AIES . . . . . . . . . . . . . . 96

5.23 Similarity values grouped by frequency in DRM . . . . . . . . . . . 96

5.24 Effectiveness (JS based) in DRM . . . . . . . . . . . . . 97

5.25 Effectiveness (JS based) in DRM2 . . . . . . . . . . . . . . 98

6.1 Hash method based on hierarchical set of topics from a given topic distribution . . . . . . . . . . . . . . . . . . . 104

6.2 Threshold-based Hierarchical Hash $(\mathrm{L}=3) \ldots \ldots \ldots$

6.3 Centroid-based Hierarchical Hash $(\mathrm{L}=3) \ldots \ldots \ldots \ldots$

6.4 Density-based Hierarchical Hash $(\mathrm{L}=3) \quad \ldots \ldots \ldots$

6.5 Topic Distribution of two documents with similarity score, based on JS,

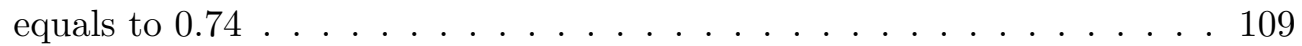

6.6 Topic Distribution of two documents with similarity score, based on JS, equals to $0.71 \ldots \ldots \ldots \ldots \ldots \ldots$

6.7 Precision at 5 (mean) of threshold-based hashing method when number of topics varies in CORDIS dataset. . . . . . . . . . . . . . 118

6.8 Precision at 5 (mean) of centroid-based hashing method when number of topics varies in CORDIS dataset. . . . . . . . . . . . . 118

6.9 Precision at 5 (mean) of density-based hashing method when number of topics varies in CORDIS dataset. . . . . . . . . . . . . . . . . . 119

6.10 Most relevant topics in related documents from using a document as query $(\mathrm{Q} 1)$ and setting topic t10 as mandatory $(\mathrm{Q} 2) \ldots \ldots \ldots \ldots$

7.1 Cross-lingual hash-expression $(\mathrm{H})$ of a document based on WordNetsynset annotations created from the top words of each topic distribution. The most relevant topics are grouped according to their importance in three levels $(h 0, h 1$ and $h 2) \ldots \ldots \ldots \ldots$ 
7.2 Graphical representation of the model that relies on the latent layer of cross-lingual topics obtained by LDA and hash functions through hierarchies of synsets. Mono-lingual approaches force to translate the documents to the same language to represent them in a unique feature space. Multi-language approaches require previously aligned topics from different languages so that documents can be represented in an equivalent feature space. Cross-lingual Synset-based approach creates a new space by combining the feature spaces of each language (i.e synsets from topn topic words). Documents are then represented in this unique space. . . . 128

7.3 topic distributions from the same document in English $\left(h_{E N}=\{(t 3062),(t 335),(t 8278)\}\right)$ and Spanish $\left(h_{E S}=\{(t 335),(t 4060),(t 5769)\}\right) \ldots \ldots \ldots \ldots \ldots$

7.4 Preparation of experiments by creating topic models for each subset of the original corpus and cross-validated with EuroVoc thesaurus . . . . . 136

7.5 Before pre-processing . . . . . . . . . . . . . . 137

7.6 After pre-processing . . . . . . . . . . . . . . . . 137

7.7 Distribution of articles by number of tokens in corpora . . . . . . . . . 137

7.8 Time needed (in milliseconds) to perform the document similarity task using similarity metrics in a corpus of $10^{4}$ synthetic documents . . . . . 142

8.1 Overview of Resources in DRInventor Platform . . . . . . . . . . . 146

8.2 The Corpus Viewer platform provides tools for the selection of evaluators or the retrieval of relevant documents (patents, scientific publications, grants and $\mathrm{R}+\mathrm{D}$ proposals for innovation evaluation). In addition, it is used for plagiarism detection, identification of double funding cases and fraud in aid grants and proposals submitted for national funding. . . . . 148

8.3 Representation of patients through topic hierarchies based on the interactions between primary-drugs and co-drugs . . . . . . . . . . . 150

8.4 Distribution of polypharmacy among people living with and without HIV according to age $($ López-Centeno et al. 2019) . . . . . . . . . . . . 151

8.5 High-level architecture of the TheyBuyForYou platform (tbfy.eu) . . . . 153

8.6 High-level workflow of a search engine and a knowledge graph through annotations created from the CORD-19 dataset . . . . . . . . . . . . 154

9.1 librAIry API usage statistics from September 2020 to February 2021 . 165 
9.2 use of librAIry resources from different operating systems from Septem-

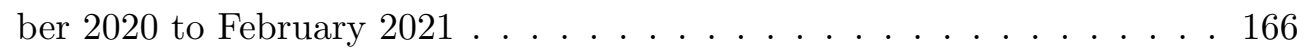

9.3 MUSE: Multilingual Unsupervised and Supervised Embeddings (Lample et al. 2018) . . . . . . . . . . . . . . . . . . . . . . . 172 


\section{List of Tables}

2.1 Number of words and tokens of publications listed in Section 1.3 when preprocessed. . . . . . . . . . . . . . . . 15

2.2 Probabilistic topics created from the collection of articles listed in Section 1.3. For each topic the five most representative words are shown together with their normalized relevance $(0-1000)$. . . . . . . . . . . . 19

2.3 Topic distributions based on the LDA model described in table 2.2 . . . 22

2.4 Kullback-Liebler divergences between the topic distributions from Table 2.3 . Note that it is not symmetric. . . . . . . . . . . . . 23

2.5 Jensen-Shannon divergences between the topic distributions from Table

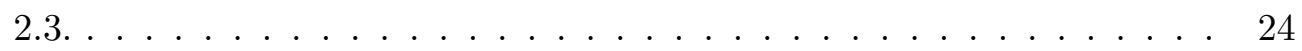

2.6 Hellinger distances between the topic distributions from Table 2.3. . . 25

2.7 S2JSD distances between the topic distributions from Table 2.3. . . . . 26

2.8 Research areas and limitations. . . . . . . . . . . . . . . . 28

2.9 Research areas and proposals. . . . . . . . . . . . . 36

3.1 Hypotheses and research dimensions. . . . . . . . . . . . . . . . 41

3.2 Open Research Challenges and Hypotheses. . . . . . . . . . . . . . 45

3.3 Research and technical objectives and their related challenges. . . . . . 49

4.1 Potential uses of a topic model. . . . . . . . . . . . . . . . 64 64

4.2 Operations offered by a topic model-as-a-service to cover potential tasks. 66

5.1 Accuracy results when comparing the most related articles using a summary (e.g. abstract, approach, background, challenge, future work or outcome), with those obtained using the full-text of the article (internalrepresentativeness $\ldots \ldots \ldots \ldots \ldots \ldots \ldots$................ 78 
5.2 Precision (JS-based) in AIES . . . . . . . . . . . . . 92

5.3 Precision (He-based) in AIES . . . . . . . . . . . . . . . 93

5.4 Recall (JS-based) in AIES . . . . . . . . . . . . . . . . . . . 93

5.5 Recall (He-based) in AIES . . . . . . . . . . . . . . . . 94

6.1 Precision at 5 (mean and median) of threshold-based (THHM), centroidbased (CHHM) and density-based (DHHM) hierarchical hashing methods on Open Research dataset using a model with 100 topics. LEVEL column indicates the number of hierarchies used. . . . . . . . . . . 111

6.2 Precision at 5 (mean and median) of threshold-based (THHM), centroidbased (CHHM) and density-based (DHHM) hierarchical hashing methods on Open Research dataset using a model with 500 topics. LEVEL column indicates the number of hierarchies used. . . . . . . . . . . . . 111

6.3 Precision at 5 (mean and median) of threshold-based (THHM), centroidbased (CHHM) and density-based (DHHM) hierarchical hashing methods on CORDIS dataset using a model with 70 topics. LEVEL column indicates the number of hierarchies used. . . . . . . . . . . . . . 112

6.4 Precision at 5 (mean and median) of threshold-based (THHM), centroidbased (CHHM) and density-based (DHHM) hierarchical hashing methods on CORDIS dataset using a model with 150 topics. LEVEL column indicates the number of hierarchies used. . . . . . . . . . . . . . . . 112

6.5 Precision at 5 (mean and median) of threshold-based (THHM), centroidbased (CHHM) and density-based (DHHM) hierarchical hashing methods on Patents dataset using a model with 250 topics. LEVEL column indicates the number of hierarchies used. . . . . . . . . . . . . . 114

6.6 Precision at 5 (mean and median) of threshold-based (THHM), centroidbased (CHHM) and density-based (DHHM) hierarchical hashing methods on Patents dataset using a model with 750 topics. LEVEL column indicates the number of hierarchies used. . . . . . . . . . . . . . . . . 114

6.7 Data size ratio used (mean and median) of threshold-based (THHM), centroid-based (CHHM) and density-based (DHHM) hierarchical hashing methods on Open Research dataset and 100 topics. . . . . . . . . . . 115 
6.8 Data size ratio used (mean and median) of threshold-based (THHM), centroid-based (CHHM) and density-based (DHHM) hierarchical hashing methods on Open Research dataset and 500 topics. . . . . . . . . . . 115

6.9 Data size ratio used (mean and median) of threshold-based (THHM), centroid-based (CHHM) and density-based (DHHM) hierarchical hashing methods on CORDIS dataset and 70 topics. . . . . . . . . . . . 116

6.10 Data size ratio used (mean and median) of threshold-based (THHM), centroid-based (CHHM) and density-based (DHHM) hierarchical hashing methods on CORDIS dataset and 150 topics. . . . . . . . . . . . 116

6.11 Data size ratio used (mean and median) of threshold-based (THHM), centroid-based (CHHM) and density-based (DHHM) hierarchical hashing methods on Patents dataset and 250 topics. . . . . . . . . . . 117

6.12 Data size ratio used (mean and median) of threshold-based (THHM), centroid-based (CHHM) and density-based (DHHM) hierarchical hashing methods on Patents dataset and 750 topics. . . . . . . . . . . 117

6.13 Number of documents related to a given one (q1) and also in a specific domain (q2) for threshold-based (thhm), centroid-based (chhm) and density-based $(\mathrm{dhhm})$ hierarchical hashing methods. . . . . . . . . . 120

7.1 Randonly selected theme-aligned topics described by top 10 words based on EUROVOC annotations from JRC-Acquis dataset . . . . . . . . . 130

7.2 Document classification performance (precision-'prec', recall-'rec' and fMeasure-'f1') of the categories-based (cat) and synset-based (syn) topic alignment algorithms in monolingual document collections . . . . . . . 133

7.3 Document classification performance (precision-'prec', recall-'rec' and fMeasure-'f1') of the categories-based (cat) and synset-based (syn) topic alignment algorithms in multi-lingual document collections . . . . . . 134

7.4 Information retrieval performance (precision@3, precision@5 and precision@10) of the categories-based (cat) and synset-based (syn) topic alignment algorithms in monolingual document collections . . . . . . 135

7.5 Information retrieval performance (precision@3, precision@5 and precision@10) of the categories-based (cat) and synset-based (syn) topic alignment algorithms in multi-lingual document collections . . . . . . 135 
7.6 Number of documents and tokens by dataset . . . . . . . . . . 136

7.7 Aggregated MAP results by metric and model . . . . . . . . . . . . . 138

7.8 MAP results by dividing the corpus into three equal subsets to train and evaluate the models in $\operatorname{English}(e n)$ and $\operatorname{Spanish}(e s) \ldots \ldots$. . . . 139

7.9 MAP results by dividing the corpus into six equal subsets to train and evaluate the models in $\operatorname{English}(e n)$ and $\operatorname{Spanish}(e s) \quad \ldots . . . . . .140$

7.10 MAP results by dividing the corpus into nine equal subsets to train and evaluate the models in $\operatorname{English}(e n)$ and $\operatorname{Spanish}(e s) \ldots \ldots$. . . . 141 


\section{Acronyms}

AI: Artificial Intelligence

AIES: Advances In Engineering Software journal dataset

ANN: Approximate Nearest Neighbour

API: Application Programming Interface

BoW: Bag-of-Words

CHHM: Centroid-based Hierarchical Hashing

CRDC: Cumulative Ranking on Dirichlet distribution- based Clustering

DHHM: Density-based Hierarchical Hashing

DR: Document Retrieval

DRM: Dirichlet Random Mixture dataset

HE: Hellinger Distance

IR: Information Retrieval

JS: Jensen Shannon divergence

KL: Kullback-Liebler divergence

LDA: Latent Dirichlet Allocation

LSA: Latent Semantic Analysis

LSH: Locality-Sensitive Hashing

MAP: Mean Average Precision

MuPTM: Multilingual Probabilistic Topic Model

NLP: Natural Language Processing 
NM: Neural Models

PLSA: Probabilistic Latent Semantic Analysis

PTM: Probabilistic Topic Model

RDC: Ranking on Dirichlet distribution-based Clustering

TDC: Trends on Dirichlet distribution-based Clustering

TF: Term Frequency

TF-IDF: Term Frequency-Inverse Document Frequency

THHM: Threshold-based Hierarchical Hashing

URI: Uniform Resource Identifier

URL: Uniform Resource Locator

VSM: Vector Space Models

WUI: Web User Interface 


\section{Chapter 1}

\section{Introduction}

Large amounts of textual documents are daily produced in digital format. More than $2 \mathrm{~K}$ blog entries have been published, $9 \mathrm{~K}$ tweets have been written, and more than $2 \mathrm{M}$ emails have been sent on the Internet every second in 2021 so far. The number of scientific publications per year increased by $8-9 \%$ in the last decade (Johnson et al. 2018). More than $1 \mathrm{M}$ papers, about two per minute, were submitted to the PubMed database, the leading database of references and abstracts on life sciences and biomedical research, during 2018. The behavior is similar in other domains. More than $168 \mathrm{~K}$ procedural documents and $3 \mathrm{~K}$ judicial notices were published in the Official Journal of the European Union $2^{2}$ in 2019. Furthermore, unlike the academic domain where articles are mostly published in English, legal documents are usually available in multiple languages. The Court of Justice of the European Union $]^{3}$ had to translate in 2019 over 1 million texts into its 24 official languages in just one year. These numbers make it difficult for an expert in the academic or legal domains to stay updated by only reading a few articles. Navigating the growing amount of textual data and exploring their content is not only necessary, but has become a crucial activity that experts must add to their daily tasks.

Document retrieval techniques are being used nowadays to facilitate text review in such big collections. Major digital publishers specialized in scientifi( 4 , technica $5^{5}$, and

${ }^{1}$ https://www.internetlivestats.com/one-second

2 https://eur-lex.europa.eu/oj/direct-access.html

3 https://curia.europa.eu

${ }^{4}$ https://www.nature.com

5 https://www.elsevier.com 
medica $\sqrt{6}$ content provide search engines to browse their collections of digital articles. Given a few keywords, a list of relevant papers is retrieved and offered for reading. Legal documents are also exploited through similar solutions. The Spanish 7 , American 8 and European $9^{9}$ intellectual property registration offices, for example, allow exploring their patent collections with search engines guided by keywords and/or categories. These categories are based on the International Patent Classification (IPC) system and are available thanks to the manual annotation of their authors and of patent officers. This classification contains approximately 70,000 codes for different technical areas. This label-based browsing has been also adopted by other academic search engines ${ }^{10}$ to organize papers by research areas, or even by evaluation tasks to cover the state-of-the-art methods 11 . A domain such as natural language processing, for example, is organized into 256 categories including 'knowledge representation', 'question-answering', 'machine translation', etc. However, the use of tags to categorize scientific papers is still insufficient for an efficient exploration and some additional text processing tasks are required to homogenize the format and criteria of these article labels and keywords. One of the main reasons that limits a widespread use of these techniques is the difficulty to identify labels that describe a work in sufficient detail.

Furthermore, not all approaches can assume that new categories will emerge. Searches guided by keywords or tags are useful when the domain is known, but when new categories appear these mechanisms may be insufficient. In scientific domains, for example, a way to identify new research areas is through periodic reviews. There are annual reports that identify the hottest and emerging specialty areas in scientific research from the last years ${ }^{12}$. The methodology used in these reports assumes that cumulatively, and over time, citations in research leave a trail that highlights progress and advancements across a range of fields. By regularly tracking these citations and unpacking the patterns and groupings of how papers are cited, in particular clusters of papers that are frequently cited together, new research areas take shape. Such reports also exist

\footnotetext{
6 https://pubmed.ncbi.nlm.nih.gov

'https://www.oepm.es

$\varepsilon$ https://www.uspto.gov/

https://www.epo.org

10 https://academic.microsoft.com

${ }^{11}$ https://paperswithcode.com

12 https://discover.clarivate.com/ResearchFronts2019_EN
} 
on patent collections to discover technology trends $\$ 3$.

In this context, identifying relationships between documents is key to know the information that they contain and facilitate their exploration. A documentary exploration does not stop when a relevant article is found, but starts from its content shaping the area of interest through its relations. Most academic ${ }^{14}$ and lega ${ }^{15}$ search engines provide a list of related documents for each text and offer navigating through them. The relationship between two documents can be based on references, when documents are cited by other: ${ }^{16}$ or are authored by an overlapping set of authors, or content, when documents share a thematic area. The articles derived from that related content can lead to more complex structures when cross-relations are considered. A document can be related to another that, in turn, is related to a third one that can be also related to the first article. This content-guided exploration helps browsing document collections by areas of interest not necessarily aligned with a list of predefined categories. A visual overview of an academic field, for example, can be provided by showing graphs of articles with related content 17 .

While these initiatives are valuable efforts to facilitate the access to large amounts of documents, they are still insufficient to make the most out of the knowledge available within the textual collection. Two types of inferences are needed: bottom-up, from documents to collections, and top-down, from document relationships to texts. The knowledge derived from a text comes from the concepts evoked by its words Griffiths et al., 2007), and the knowledge derived from a document collection emerges from the relationships between its texts (Kenter and Rijke, 2015). Both require understanding the meaning, the semantics, of texts at different levels. Semantic awareness is desirable to interpret the relationships and guide the exploration. The focus is on why some texts are related and what concepts are key to those relationships. But analyzing and comparing texts on a large scale also requires addressing some challenges imposed by external conditions that have appeared in recent years:

- Efficiency: The algorithms, besides being accurate enough, must be also efficient in order to be applied on a large scale. Brute-force techniques cannot be applied

\footnotetext{
13 https://www.wipo.int/tech_trends

14 https://www.semanticscholar.org

15 https://patents.google.com

16 http://citationgecko.com

17 https : //www . connectedpapers . com
} 
to compare all items in large corpus.

- Explainability: The relations among documents must be explained in such a way that they provide knowledge about the content of the texts. It is not enough to determine that one text is related to another, it is necessary to explain why it is so.

- Complexity: The increasing variety of themes, in ever growing collections, has forced a reconsideration of the way to compare their documents. The operations required to compute each comparison should be simplified as much as possible.

- Multilinguality: The increasing availability of texts written in different natural languages also makes it necessary to perform comparisons in multilingual collections. External translation systems cannot been considered as the only applicable solution, since they increase processing costs and potentially introduce bias in the relationships that are obtained. Solutions that relate texts without necessarily translate them are desirable.

In our work we aim at facilitating the exploration of large collections of documents written in multiple languages. We address the problem of comparing them on a large scale while enabling a semantic-aware exploration through their content. Our proposal automatically finds thematic associations between texts using probabilistic models to describe their content through topics, and organizes document collections so that they can be efficiently and transparently browsed through the related content regardless of their language.

\subsection{Contributions}

The following contributions are presented in this thesis:

- A Scalable Framework for Probabilistic Topic Modeling: We design and implement a text processing platform that supports the creation of probabilistic topic models by distributing operations among functional units. Based on this abstraction, we implement a framework that becomes the foundation of this thesis research, which is used as a tool for supporting performance analysis and algorithm design. 
- A Publishing Model for Probabilistic Topic Models: We propose a unified mechanism to publish and (re)use topic models as Web services that are available from online repositories, so as to facilitate the exploitation of probabilistic topic models.

- Hierarchical Thematic Annotations for High-dimensional Topic Models: To study the problem of representativeness in high dimensional topic models, we exploit the relationships between texts derived from their topic distributions. We show how the distances vary between the same texts when the dimensions of the model change, and how less representative topics can influence their calculation. Our analytical and experimental results show that the more topics are available in the model, the less representative the distance measurements based on densities are. We identify hierarchies in the topic distributions that maintain their representativeness regardless of the dimensionality of the model, and without losing the ability to measure distances. We propose a method to annotate texts using topic hierarchies, and a distance metric based on these hierarchical representations.

- Support for Large-scale Document Similarity Comparisons: We present an efficient mechanism to index and retrieve related documents using hierarchical annotations. It facilitates the exploration of a collection by the themes inferred from its texts.

- Identify Cross-lingual Document Relations: We introduce a technique to transform probabilistic topics from different languages into a single representation space based on shared concepts where texts can be thematically related regardless of the language used.

\subsection{Thesis Structure}

The thesis is structured as follows:

Chapter 2 describes the main concepts handled throughout the thesis, analyses the state of the art and identifies the main limitations. Chapter 3 presents the research problems and hypotheses that guide our work, as well as assumptions and restrictions 
and details the methodology that has been followed in this research. Chapter 4 describes the software architecture proposed to analyze large document collections and the format suggested to distribute and reuse the topic models built in this thesis. Chapter 5 details our proposed text annotation algorithm to organize probabilistic topics into hierarchical levels according to their relevance. Chapter 6 shows how to store and search documents efficiently from large collections when they are annotated with topic hierarchies. Chapter 7 explains the method to relate multilingual texts from their main topics without requiring any prior knowledge between the languages. Chapter 8 describes real-world projects where the results from this thesis have been used. Finally, Chapter 9 presents the conclusions and introduce the future lines of work. Where relevant, evaluations have been included in the corresponding chapters where the specific contributions are presented.

\subsection{Publications}

The following publications support the research work presented in this thesis:

\section{- Chapter 4: Creation and Publication of Probabilistic Topic Models:}

- Carlos Badenes-Olmedo, José Luis Redondo-Garcia, and Oscar Corcho. Distributing Text Mining tasks with librAIry. Proceedings of the 17th ACM Symposium on Document Engineering (DocEng). Valletta, Malta. $201 \%$. This paper describes the architecture and technological features of librAIry, our framework for processing texts and creating probabilistic topic models.

- Victoria Kosa, Alyona Chugunenko, Eugene Yuschenko, Carlos BadenesOlmedo, Vadim Ermolayev, and Aliaksandr Birukou. Semantic saturation in retrospective text document collections. Information and Communication Technologies in Education, Research, and Industrial Applications (ICTERI) PhD Symposium, vol. 1851, pages 1-8. CEUR-WS. 2017. The work presented in this paper makes use of the text-processing module proposed by librAIry to retrieve multi-word terms from a document collection.

- Victoria Kosa, David Chaves-Fraga, Dmitriy Naumenko, Eugene Yuschenko, Carlos Badenes-Olmedo, Vadim Ermolayev, Aliaksandr Birukou, Nick Bassiliades, Hans-Georg Fill, Vitaliy Yakovyna, Heinrich C. Mayr, Mykola 
Nikitchenko, Grygoriy Zholtkevych, and Aleksander Spivakovsky. CrossEvaluation of Automated Term Extraction Tools by Measuring Terminological Saturation. Information and Communication Technologies in Education, Research, and Industrial Applications, pages 135-163. Springer International Publishing. 2018. The distributed document processing and representation model used by librAIry is improved during this work to support the extraction of the most relevant terms from a collection of scientific articles.

- Chapter 5 Explainable Topic-based Associations:

- Carlos Badenes-Olmedo, José Luis Redondo-García, and Oscar Corcho. Efficient Clustering from Distributions over Topics. Proceedings of the 9th International Conference on Knowledge Capture (K-CAP), Article 17, 1-8. Association for Computing Machinery, Austin, TX, USA. 2017. This article provides the basis for transforming probabilistic topic distributions into expressions that group related content.

- Carlos Badenes-Olmedo, José Luis Redondo-Garcia, and Oscar Corcho. An initial Analysis of Topic-based Similarity among Scientific Documents based on their Rhetorical Discourse Parts. Proceedings of the 1st Workshop on Enabling Open Semantic Science (SemSci) co-located with 16th International Semantic Web Conference (ISWC 2017), 15-22. Vienna, Austria. 2017. This work demonstrates the need to use full texts to relate content from their topic distributions, since the use of abstracts of scientific texts is shown to be less accurate than other longer sections.

- Chapter 6: Large-scale Comparisons of Topic Distributions:

- Carlos Badenes-Olmedo, José Luis Redondo-García, and Oscar Corcho. Large-scale Semantic Exploration of Scientific Literature Using Topic-based Hashing Algorithms. Semantic Web journal, vol. 11, no. 5, pp. 735-750. 2020. This paper describes the key algorithm of this thesis to reduce probabilistic topic distributions into hierarchical expressions that relate content without losing topic information. 


\section{- Chapter 7: Cross-lingual Document Similarity:}

- Carlos Badenes-Olmedo, José Luis Redondo-García, and Oscar Corcho. Scalable Cross-lingual Document Similarity through Language-specific Concept Hierarchies. Proceedings of the 10th International Conference on Knowledge Capture (K-CAP). Association for Computing Machinery, 147-153. Marina Del Rey, CA, USA. 2019. In this paper we describe probabilistic topics using concept-based representations instead of words. Multilingual topics are automatically aligned to create a single representation space across languages.

- Carlos Badenes-Olmedo, José Luis Redondo-García, and Oscar Corcho. Legal document retrieval across languages: topic hierarchies based on synsets. Proceedings of the 1st Workshop on Iberlegal co-located with 32nd International Conference on Legal Knowledge and Information Systems organized by the Foundation for Legal Knowledge Based Systems (JURIX). Madrid, Spain. 2019. The work presented in this paper evaluates the conceptual representation of multilingual topics to relate legal documents in several languages: English, Spanish, French and Portuguese.

\section{- Chapter 8: Experiments:}

- Beatriz López-Centeno, Carlos Badenes-Olmedo, Ángel Mataix-Sanjuan, Katie McAllister, José M Bellón, Sara Gibbons, Pascual Balsalobre, Leire Pérez-Latorre, Juana Benedí, Catia Marzolini, Ainhoa Aranguren-Oyarzábal, Saye Khoo, María J Calvo-Alcántara, Juan Berenguer. Polypharmacy and Drug-Drug Interactions in People Living With Human Immunodeficiency Virus in the Region of Madrid, Spain: A Population-Based Study. Clinical Infectious Diseases. vol. 71,2, 353-362. 2020. In this work we exploit the ability of librAIry to relate content described by hierarchical representations. In a medical domain, patients are grouped according to the drugs used in their treatments and their potential interactions.

- Ahmet Soylu, Oscar Corcho, Brian Elvesaeter, Carlos Badenes-Olmedo, Francisco Yedro, Matej Kovacic, Matej Posinkovic, Ian Makgill, Chris Taggart, Elena Simperl, Till C. Lech, and Dumitru Roman. Enhancing Public 
Procurement in the European Union through Constructing and Exploiting an Integrated Knowledge Graph. Proceedings of the 19th International Semantic Web Conference (ISWC). 2020. This paper presents the work done to relate multilingual European regulations to public contracts through their hierarchical topic-based representations. A document search engine that leverages this facility is built.

- Ahmet Soylu, Oscar Corcho, Brian Elvesaeter, Carlos Badenes-Olmedo, Francisco Yedro, Matej Kovacic, Matej Posinkovic, Ian Makgill, Chris Taggart, Elena Simperl, Till C. Lech, and Dumitru Roman. TheyBuyForYou Platform and Knowledge Graph: Expanding Horizons in Public Procurement with Open Linked Data. Semantic Web journal, (under minor revision). 2021. This article details the construction of an open linked data platform that uses our algorithms to automatically link textual data across several European languages.

- Carlos Badenes-Olmedo, David Chaves-Fraga, Maria Poveda-Villalon, Ana Iglesias-Molina, Pablo Calleja, Socorro Bernardos, Patricia MartínChozas, Alba Fernández-Izquierdo, Elvira Amador-Dominguez, Paola EspinozaArias, Luis Pozo, Edna Ruckhaus, Esteban Gonzalez-Guardia, Raquel Cedazo, Beatriz Lopez-Centeno, and Oscar Corcho. Drugs4Covid: Drug-driven Knowledge Exploitation based on Scientific Publications. arXiv e-prints:2012.01953. 2020. In the context of creating a knowledge graph describing the drugs used to treat COVID-19, this article shows the work to adapt librAIry to infer relationships between drugs from their mentions in scientific articles. 


\section{Chapter 2}

\section{State of the Art}

In this chapter the main concepts that will be used throughout the rest of the thesis are introduced. We analyze the current state of the art and limitations when exploring large multilingual document collections. First, the tasks related to processing the texts and enabling a semantic-aware exploration of the corpus are described. Then, an overview of the existing methods and techniques that allow performing these tasks is provided. And finally, for each research area involved, the limitations that must be addressed to achieve the ultimate goal of facilitating documentary exploration are presented.

\subsection{Information Retrieval}

The analysis of human-readable documents is a well-known problem in Artificial Intelligence (AI) in general, and in the Information Retrieval (IR) and Natural Language Processing (NLP) fields in particular. As an academic field of study, information retrieval might be defined as finding documents of an unstructured nature, usually text, that satisfy an information need from within large collections (Manning et al., 2008). Hundreds of millions of people perform some information retrieval task everyday when they use a web search engine or search for an email in their mailbox. Information retrieval is fast becoming the dominant form of information access, surpassing traditional database searching where identifiers are needed to obtain results.

There are some key concepts in this area. The records that IR addresses are called documents, and they are retrieved from an organized repository, most commonly called collection or corpus. It is not restricted to static collections. The collection may 
be a stream of texts, e.g., scientific publications, patents, news dispatches, that are created periodically. IR focuses on retrieving documents from a collection based on their content. A request, typically called a query, may specify desired characteristics of the documents to be retrieved, e.g., The documents should be about 'Text Similarity' and their author must be 'Badenes'. In this example, the query asks for documents whose content (the unstructured part) is about a certain topic and whose author (a structured part) has a specified value.

Unstructured Content means that there is no well-defined syntax for a given document, let alone the arrangement of words and phrases to create well-formed sentences in a language that all documents share. To the extent that documents share a syntax, there is no well-defined semantics associated with each syntactic component (Greengrass, 2000). Now, think about how data is structured in a database. All records of a given type have the same syntax, e.g., all rows in a table of a relational database will have the same columns (Salton and McGill, 1983). Moreover, each component of a record will have a definite meaning (i.e. semantics) and a given component of a given record type will have the same semantics in every record of that type. The practical effect is that given the name of a component, a search engine can use the syntax to find the component in a given record and retrieve its contents (i.e. its value). Similarly, given a component and a value, the search engine can find records such that the given component contains the given value. For example, a relational database can be asked to retrieve the contents of the year column of a Thesis table in an Academic database. The search engine knows how to find the Thesis table within the Academic database, and how to find the year column within each record of the Thesis table. And every year column within the Thesis table will have the same semantics, i.e., the year of publication of a thesis.

But the column name year may not be sufficient to identify the column. A Course table in the same or a different database may also have a year column. Hence in general, it may be necessary to specify a path, e.g., database name, table name,column name, to uniquely identify the syntactic component to the search engine. However, the syntax of a well-structured database is such that it is always possible to specify a given syntactic component uniquely and hence it is always possible for the search engine to find all occurrences of a given component. If the given component has a definite semantics, 
then it is always possible for the search engine to find data with that semantics, e.g., to find the years of all the theses.

By contrast, in a collection of unstructured natural language documents, there is no well-defined syntactic position where a search engine could find data with a given semantics, e.g., the years of theses. In a random collection of documents, there is no guarantee that they are all about the same topic, e.g., theses. Even if it is known that the documents are all about theses, there is no simple well-defined way of knowing where the year occurs in a given document, e.g., in what sentence or even in what paragraph. This is exactly what is meant by unstructured texts.

An information retrieval engine may use the query to classify the documents in a collection, returning to the user a subset of documents that satisfy some classification criterion. Documents that satisfy the query are said to be relevant. The higher the proportion of documents returned to the user that judges as relevant, the better the classification criterion. Alternatively, the search engine may rank the documents in a given collection. Document $D_{1}$ ranks higher with respect to a given query $Q$ than document $D_{2}$, what means that $D_{1}$ is more likely to satisfy $Q$ than $D_{2}$. Two measures of IR success, both based on the concept of relevance, are widely used: precision and recall. Precision is defined as the ratio of relevant items retrieved to all items retrieved, or the probability given that an item is retrieved that it will be relevant. Recall is defined as, the ratio of relevant items retrieved to all relevant items in a collection, or the probability given that an item is relevant that it will be retrieved (Saracevic, 1995).

\subsection{Techniques for Document Retrieval}

There are two major categories of IR technology and research: semantic and statistical. Semantic approaches attempt to implement some degree of syntactic and semantic analysis. They try to reproduce to some degree the understanding of the natural language text that a human user would provide. In statistical approaches, the documents that are retrieved or that are highly ranked are those that match the query most closely in terms of some statistical measure. The work presented in this thesis follows this second approach.

Statistical approaches break documents and queries into tokens. These tokens are the population that is counted and measured statistically. Most commonly, the to- 
kens are words (or combination of adjacent words or characters) that occur in a given query or collection of documents and often require pre-processing. Words are reduced to a common base form by using a heuristic process that removes affixes, stemming, or by returning its dictionary form, lemma (Porter, 1997). The objective is to eliminate the variation that arises from the occurrence of different grammatical forms of the same word, e.g., "program", "programming", "programs", and "programmed" should all be recognized as forms of the same word, "program". Another common form of pre-processing is the elimination of common words that have little power to discriminate relevant from non-relevant documents,e.g., "the", "a", "it". Hence, IR engines are usually provided with a stop-list of such noise words. Note that both stemming/lemma and stopwords are language-dependent. Once all terms have been pre-processed, numerical weights are assigned to each them. The same term may have a different weight in each distinct document in which it occurs. The weight is usually a measure of how effective the given term is likely to be in distinguishing a document from other documents in the collection, and is often normalized to be a fraction between zero and one. Statistical approaches fall into the following categories: boolean, vector space and probabilistic.

An illustrative example ${ }^{18}$ may help to better understand these techniques. The publications listed in Section 1.3 are used as a sample collection for applying information retrieval techniques. Documents are first pre-processed as described above to transform their texts into terms. Typically these terms are referred to as tokens. Let's see the process by analyzing the following sentence taken from one of the documents: "Probabilistic Topic Models reduce that feature space by annotating documents with thematic information". Each word, identified using whitespace characters, is normalized using its dictionary form (stemming could also have been performed in this step). The sentence is then transformed into a list of lemmatized words: "['Probabilistic', 'Topic', 'Models', 'reduce', 'that', 'feature', 'space', 'by', 'annotate', 'document', 'with', 'thematic', 'information']". Some words have changed and others have remained unchanged. For example, 'annotating' is reduced to 'annotate' and 'documents' to 'document'. However, 'Models' remains unchanged. The reason is that it is considered a proper noun since it starts with a capital letter. Once the words have been normalized, the words with less informative capacity are eliminated (e.g. 'that', 'by' and 'with'). They usually

\footnotetext{
${ }^{18}$ https://github.com/cbadenes/phd-thesis/blob/master/notebooks/soa.ipynb
} 
belong to a stop-word list associated with a given language, but can also be domainspecific. The final list of terms are the tokens used to describe each of the documents. Table 2.1 shows the number of words and tokens of each document when preprocessed.

\begin{tabular}{|c|c|c|c|}
\hline Id & Title & Words & Tokens \\
\hline$D_{0}$ & $\begin{array}{l}\text { Cross-Evaluation of Term Extraction Tools by Measuring } \\
\text { Terminological Saturation }\end{array}$ & 12,954 & 6,342 \\
\hline$D_{1}$ & $\begin{array}{l}\text { Enhancing Public Procurement in the European Union } \\
\text { through Constructing and Exploiting an Integrated } \\
\text { Knowledge Graph }\end{array}$ & 5,827 & 3,406 \\
\hline$D_{2}$ & $\begin{array}{l}\text { Drugs4Covid: Making drug information available from } \\
\text { scientific publications }\end{array}$ & 5,417 & 3,260 \\
\hline$D_{3}$ & Distributing Text Mining tasks with librAIry & 2,448 & 1,477 \\
\hline$D_{4}$ & $\begin{array}{l}\text { Large-Scale Semantic Exploration of Scientific Literature } \\
\text { using Topic-based Hashing Algorithms }\end{array}$ & 9,041 & 5,604 \\
\hline$D_{5}$ & $\begin{array}{l}\text { An initial Analysis of Topic-based Similarity among } \\
\text { Scientific Documents based on their Rhetorical Discourse } \\
\text { Parts }\end{array}$ & 2,641 & 1,425 \\
\hline$D_{6}$ & Efficient Clustering from Distributions over Topics & 5,346 & 2,986 \\
\hline$D_{7}$ & $\begin{array}{l}\text { Legal Documents Retrieval Across Languages: Topic } \\
\text { Hierarchies based on synsets }\end{array}$ & 1,445 & 787 \\
\hline$D_{8}$ & $\begin{array}{l}\text { Scalable Cross-lingual Document Similarity through } \\
\text { Language-specific Concept Hierarchies }\end{array}$ & 4,602 & 2,963 \\
\hline$D_{9}$ & $\begin{array}{l}\text { Potentially inappropriate medications in older adults living } \\
\text { with HIV }\end{array}$ & 3,087 & 2,018 \\
\hline$D_{10}$ & $\begin{array}{l}\text { Semantic Saturation in Retrospective Text Document } \\
\text { Collections }\end{array}$ & 7,700 & 1,753 \\
\hline
\end{tabular}

Table 2.1: Number of words and tokens of publications listed in Section 1.3 when preprocessed.

In a boolean approach, the query is formulated as a boolean combination of terms. Documents are reduced to true or false representations for each word depending 
on whether they contain it or not. A conventional boolean query uses the classical operators AND, OR, and NOT. The query " $t_{1}$ AND $t_{2}$ " is satisfied by a given document $D_{1}$ if and only if $D_{1}$ contains both terms $t_{1}$ and $t_{2}$. Similarly, the query " $t_{1}$ OR $t_{2}$ " is satisfied by $D_{1}$ if and only if it contains $t_{1}$ or $t_{2}$ or both. The query " $t_{1}$ AND NOT $t_{2}$ " satisfies $D_{1}$ if and only if it contains $t_{1}$ and does not contain $t_{2}$. In the above example, the query needed to find the articles dealing with topic hierarchies or multilingualism would be: "multilingual OR (topic AND hierarchy)" (note the normalization of terms). More complex boolean queries can be built from these operators and evaluated according to the classical rules of boolean algebra. Such a boolean query is either true or false. Correspondingly, a document either satisfies such a query, i.e. is relevant, or does not satisfy it, i.e. is non-relevant. For the above query, documents $D_{1}, D_{4}, D_{6}, D_{7}$ and $D_{8}$ are found relevant. However no ranking is possible using this representation, which is a significant limitation for this approach (Harmon, 1996).

Vector space models (VSM) (Salton and McGill, 1983) were proposed to represent texts as vectors where each entry corresponds to a different term and the number at that entry corresponds to how many times that term is present in the text. The objective was twofold: on the one hand, making document collections manageable since we move from having lots of terms for each text to only one vector per document with a defined dimension; on the other hand, having representations based on metric spaces where calculations can be made, for example comparisons by measuring vector distances. The definition and number of dimensions for each vector are key aspects in a VSM. Based on the use of this type of model, traditional document retrieval tasks over collections of textual documents highly rely on individual features like term frequencies (TF) (Hearst and Hall, 1999). A representational space is created where each term in the vocabulary is projected by a separate and orthogonal dimension. The relevance of the documents according to a given query can be calculated by adding the frequencies of the query terms for each document. Thus, the search for articles dealing with topic hierarchies or multilingualism return the sorted list of relevant documents: (1) $D_{4},(2) D_{8},(3) D_{6},(4) D_{7}$ and (5) $D_{1}$. But in this approach all terms in a document are treated as equally descriptive. To overcome this limitation, Term-Frequency Inverse-Document Frequency (TF-IDF) (Lee, 1995) relativizes the relevance of each term with respect to the entire corpus. TF-IDF calculates the importance of a term for a document, based on the number of times the term appears in the document itself 
(term frequency - TF) and the number of documents in the corpus, which contain the term (document frequency - DF). The above query, taking into account this representation, slightly varies the order of relevance of the documents: (1) $D_{8},(2) D_{7},(3) D_{4}$, (4) $D_{6}$ and (5) $D_{1}$. $D_{8}$ and $D_{7}$ are now the most relevant documents because, although the query terms are less frequent than in $D_{4}$, their relative frequency (considering the length of the articles) is higher. However the absence of semantic information and the high-number of dimensions are the main drawbacks of these approaches that lead to the emergence of other techniques. In the above example, with a collection of only 11 documents, the dimension of the vectors is equal to 6,182 (i.e. the number of unique tokens in the whole corpus).

New ways of characterizing documents appeared more recently based on models describing the main themes covered in the corpus. Supervised approaches rely on external knowledge, e.g. taxonomies, thesaurus or ontologies, to identify these subjects (Garcia-Silva and Gómez-Pérez, 2021; Kandimalla et al., 2021; Salatino et al., 2019), while unsupervised approaches use only the content of the documents to describe them (Litschko et al. 2021; Wei and Guo, 2019). Among them, text embedding proposes transforming texts into low-dimensional vectors by prediction methods based on (i) word sequences or (ii) bag-of-words. The first approach assumes words with similar meanings tend to occur in similar contexts. It considers that word order is relevant, and is based on Neural Models (NM) that learn word vectors from pairs of target and context words. Context words are taken as words observed to surround a target word. Document vectors are usually created by taking the word vectors they contain or by considering them as target and context items. Skip-gram with negative sampling (Word2Vec) (Mikolov et al., 2013) and Global Vectors (GloVe) (Pennington et al., 2014 ) are indeed the most popular methods to learn word embeddings due to its training efficiency and robustness (Levy et al., 2015). Figure 2.1 shows the two-dimensional projection of the example articles described by word embeddings. In particular, document representations are calculated via distributed memory using the Paragraph Vector (Le and Mikolov, 2014) algorithm. The original dimensions of the vector space were reduced by Principal Component Analysis (PCA) to the dimensions of the graph. This representation brings together documents that share not only the same vocabulary, but also the way of using it, since it takes into account sequences of words, and separates documents that use different relevant terms or in different ways. For this reason, 
document $D_{0}$ appears clearly distanced from the rest, since it is mainly focused on saturation measures from term collections. And the same happens with document $D_{7}$, which presents a specific case in the legal domain.

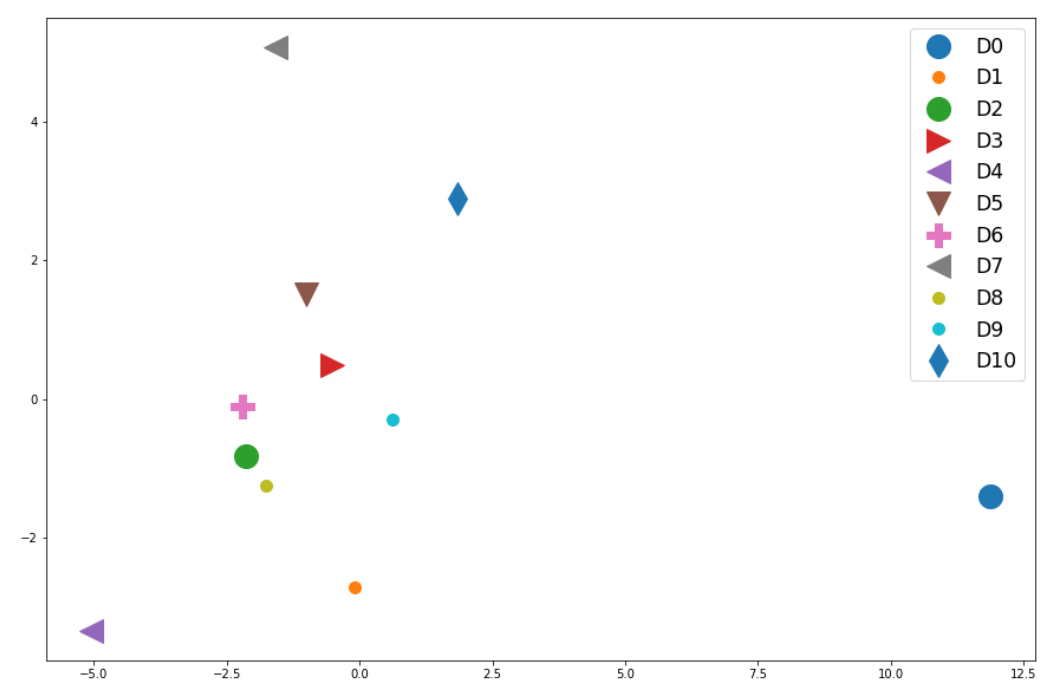

Figure 2.1: Documents listed in Section 1.3 described by word embeddings and projected in a two-dimensional space by PCA.

The second approach does not consider the order of the words to be relevant, but their frequency. It assumes words with similar meanings will occur in similar documents, and avoids considering the same terms or the same word sequences to relate documents. Topic models (Deerwester et al. 1990) are the main method that are based on this approach. Probabilistic Topic Models (PTM) (Blei et al., 2003; Hofmann, 2001) are statistical methods based on bag-of-words that analyze the words of the original texts to discover the themes that run through them, how those themes are connected to each other, or how they change over time. PTM do not require any prior annotations or labeling of the documents. The topics emerge, as hidden structures, from the analysis of the original texts. These structures are topic distributions, per-document topic distributions or per-document per-word topic assignments. In turn, a topic is a distribution over terms that is biased around those words associated to a single theme. To better understand what topic means in this context, table 2.2 shows the topics that have emerged when creating a topic model from the collection of example publications. Each topic is described by its five most representative words, i.e., those words most present in the documents that mainly contain each topic. 


\begin{tabular}{|c|c|c|c|c|}
\hline Topic 0 & Topic 1 & Topic 2 & Topic 3 & Topic 4 \\
\hline topic (27) & drug (15) & term $(21)$ & synset (9) & document (1) \\
\hline document (20) & disease (9) & collection (11) & topic $(8)$ & topic $(1)$ \\
\hline base $(17)$ & old $(6)$ & document (10) & document (8) & term $(1)$ \\
\hline $\begin{array}{l}\text { distribution } \\
(8)\end{array}$ & PLWH (5) & value (9) & lingual (7) & base (1) \\
\hline model (8) & medication (5) & saturation (9) & category (6) & collection (1) \\
\hline
\end{tabular}

Table 2.2: Probabilistic topics created from the collection of articles listed in Section 1.3 For each topic the five most representative words are shown together with their normalized relevance (0-1000).

This interpretable hidden structure annotates each document in the collection and these annotations can be used to perform deeper analysis about relationships between documents. Topic-based representations bring a lot of potential when applied over different IR tasks, as evidenced by works in different domains such as scholarly (Gatti et al., 2015), health (Hsin-Min et al., 2016; Nzali et al., 2017), legal (Greene and Cross, 2016, O'Neill et al., 2017), news (He et al., 2017) and social networks (Cheng et al., 2014). Moreover, some hybrid proposals have been recently used to model topics from word embeddings (Dieng et al. 2020). Following the previous example, Figure 2.2 shows the articles projected in a two-dimensional space from their probabilistic topicbased representations. Unlike the distribution shown in Figure 2.1, several documents now appear at the same position (e.g. $D_{2}-D_{9}$ and $D_{3}-D_{4}-D_{6}-D_{8}$ ). There are topics that are strongly present in some documents to differentiate them from the others. Thus, topic 1 groups documents $D_{2}$ and $D_{9}$ since it is related to the health domain, and topic 0 groups documents $D_{3}, D_{4}, D_{6}$ and $D_{8}$ that are clearly focused on the efficient clustering of documents by probabilistic topics. The rest of papers have a more balanced distribution of topics, indicating that they are less specific in a given domain.

Since the bag-of-words approach does not consider word sequences to relate documents, but only word frequencies, similarity relationships are stronger. Documents are related because they use the same words, regardless of their order. When topic models 


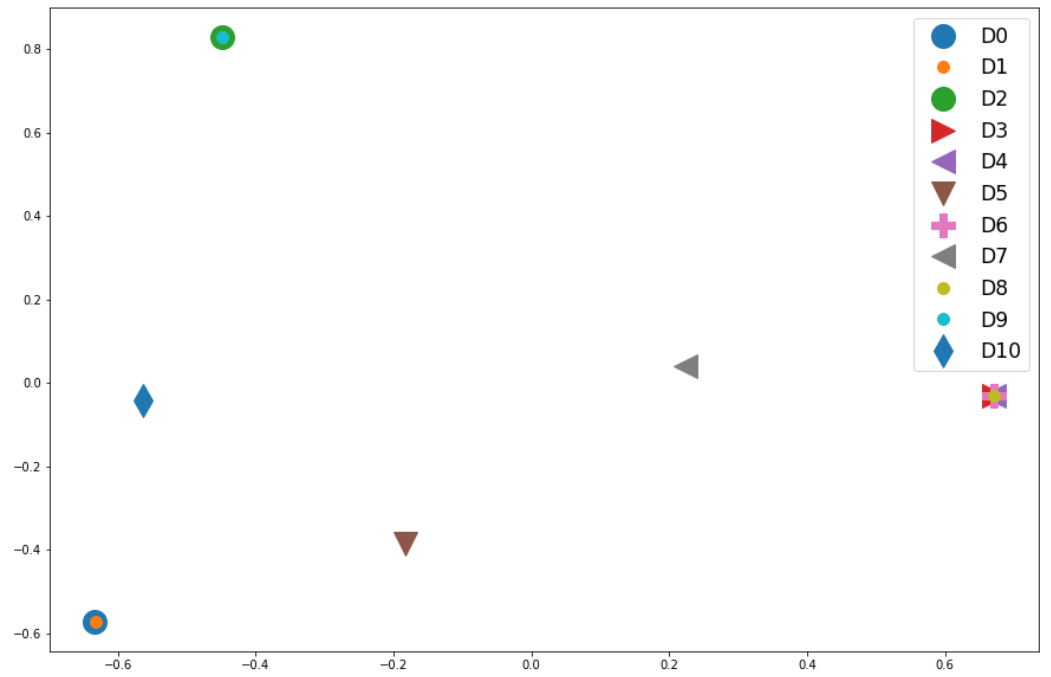

Figure 2.2: Documents listed in Section 1.3 described by probabilistic topics (Table 2.2) and projected in a two-dimensional space by PCA.

are also used (i.e. words are grouped by relevance according to thematic information), relationships are based on topic presence, not on the use of the same words. Documents are related if they use words that are relevant from the same topics, even if the words are different. Taking this assumption into account, topic modeling provides an algorithmic solution to organize and annotate large collections of textual documents according to set of words. Our work is based on this approach since we are not only interested in representing and relating words and texts, but we also look for structures that allow considering knowledge about a document collection.

\subsection{Techniques for Probabilistic Topic Models}

The simplest generative topic model proposed in the state of the art is Latent Dirichlet Allocation (LDA) (Blei et al., 2003). Along with Latent Semantic Analysis (LSA) (Deerwester et al., 1990) and Probabilistic Latent Semantic Analysis (pLSA) (Hofmann, 2001) they are part of the field known as topic modeling. They are well-known latent variable models for high dimensional data, such as the bag-of-words representation for textual data or any other count-based data representation. They try to capture the intuition that documents can exhibit multiple themes. All the documents in a collection share the same set of topics, but each document exhibits these topics in a different 
proportion. Each word in each document is drawn from one of the topics, where the selected topic is chosen from the per-document distribution over topics. Texts are described as a vector of counts with $W$ components, where $W$ is the number of words in the vocabulary. Each document in the corpus is modeled as a mixture over $K$ topics, and each topic $k$ is a distribution over the vocabulary of $W$ words. Formally, a topic is a multinomial distribution over words of a fixed vocabulary representing some concept. Depending on the function used to describe that distribution there are different algorithms to create topic models. LSA and pLSA propose a singular value decomposition. However, they have over-fitting issues. Influenced by the generative Bayesian framework LDA suggests the use of a Dirichlet function as a continuous multivariate probability distribution parameterized by a vector of positive reals whose elements sum to 1 . It is continuous because the relative likelihood for a random variable to take on a given value is described by a probability density function, and is multivariate because it has a list of variables with unknown values. In fact, the Dirichlet distribution is the conjugate prior of the categorical distribution and multinomial distribution and allows LDA to infer topic distributions in texts that have not been used during training, unlike LSA and pLSA.

In order to train a LDA model it is necessary to provide a fixed number of topics across the corpus. Each topic is drawn from a Dirichlet distribution with parameter $\beta$, while each document's mixture is sampled from a Dirichlet distribution with parameter $\alpha$. These two priors, $\alpha$ and $\beta$, set the probability that a document or a word, respectively, contain more than one topic. Along with the number of topics they are also known as hyper-parameters of an LDA model and they have to be tuned when training a model. There are other parameters common to learning models, such as the number of iterations through the corpus when inferring the topic distribution, or related to the online learning mode of LDA (Hoffman et al., 2010), for example the number of documents to be iterated through for each update, that can also be fine-tuned. However $\alpha$, $\beta$ and the number of topics are the ones that mainly define the behavior of the model for distributing the topics and therefore crucial when creating the model. Table 2.3 shows the topic distributions of the example corpus. An LDA model was trained with 5 topics to cover not only the 4 research areas of this thesis, but also experimentation; $\alpha$ equals to 1.0 (higher than usual) because the documents are likely to contain more than one topic; and $\beta$ equals to 0.01 to force to distribute the words among topics in 
a non-homogeneous way. Given the topics in Table 2.2 , it can be seen how documents present strongly unbalanced distributions with respect to some of the topics. The small size of the corpus and the specialization of the articles causes this behavior.

\begin{tabular}{cccccc}
\hline Document & Topic 0 & Topic 1 & Topic 2 & Topic 3 & Topic 4 \\
\hline \hline$D_{0}$ & 0.00006 & 0.00004 & $\mathbf{0 . 9 9 9 8 6}$ & 0.00002 & 0.00002 \\
\hline$D_{1}$ & 0.00012 & 0.00007 & $\mathbf{0 . 9 9 9 7 3}$ & 0.00004 & 0.00004 \\
\hline$D_{2}$ & 0.00227 & $\mathbf{0 . 9 9 7 6 0}$ & 0.00005 & 0.00004 & 0.00004 \\
\hline$D_{3}$ & $\mathbf{0 . 9 9 9 5 4}$ & 0.00016 & 0.00012 & 0.00010 & 0.00008 \\
\hline$D_{4}$ & $\mathbf{0 . 9 9 9 8 8}$ & 0.00004 & 0.00003 & 0.00003 & 0.00002 \\
\hline$D_{5}$ & 0.34434 & 0.00017 & $\mathbf{0 . 6 5 5 3 1}$ & 0.00010 & 0.00008 \\
\hline$D_{6}$ & $\mathbf{0 . 9 9 9 7 7}$ & 0.00008 & 0.00006 & 0.00005 & 0.00004 \\
\hline$D_{7}$ & 0.40842 & 0.00030 & 0.00023 & $\mathbf{0 . 5 9 0 9 0}$ & 0.00015 \\
\hline$D_{8}$ & $\mathbf{0 . 9 9 9 7 7}$ & 0.00008 & 0.00006 & 0.00005 & 0.00004 \\
\hline$D_{9}$ & 0.00017 & $\mathbf{0 . 9 9 9 6 1}$ & 0.00009 & 0.00007 & 0.00006 \\
\hline$D_{10}$ & 0.00021 & 0.37821 & $\mathbf{0 . 6 2 1 4 3}$ & 0.00008 & 0.00007 \\
\hline \hline
\end{tabular}

Table 2.3: Topic distributions based on the LDA model described in table 2.2 .

Topic models are not restrictive clustering models where each document is assigned to one cluster, but allow documents to exhibit multiple topics. The topics covered in a set of documents are discovered from the own corpus and feature vectors are topic distributions expressed as vector of probabilities.

\subsubsection{Document Similarity Calculation based on LDA}

Since documents are described by topic distributions, the semantic similarity between two texts is based on the distance of their vector representations of the topics, which can be also seen as two probability mass functions. A commonly used metric is the Kullback-Liebler (KL) divergence (Kullback, 1968, Kullback and Leibler, 1951):

$$
K L(P, Q)=\sum_{i=1}^{K} p\left(x_{i}\right) \log \frac{p\left(x_{i}\right)}{q\left(x_{i}\right)}
$$


where $K$ is the number of topics and $p, q$ are the topics distributions.

\begin{tabular}{cccccccccccc}
\hline & $D_{0}$ & $D_{1}$ & $D_{2}$ & $D_{3}$ & $D_{4}$ & $D_{5}$ & $D_{6}$ & $D_{7}$ & $D_{8}$ & $D_{9}$ & $D_{10}$ \\
\hline \hline$D_{0}$ & 0.0 & 0.0 & 9.81 & 9.02 & 10.36 & 0.42 & 9.73 & 8.4 & 9.72 & 9.34 & 0.48 \\
\hline$D_{1}$ & 0.0 & 0.0 & 9.81 & 9.02 & 10.36 & 0.42 & 9.73 & 8.39 & 9.72 & 9.34 & 0.47 \\
\hline$D_{2}$ & 10.19 & 9.55 & 0.0 & 8.69 & 10.04 & 8.66 & 9.41 & 8.08 & 9.41 & 0.0 & 0.97 \\
\hline$D_{3}$ & 9.76 & 9.02 & 6.07 & 0.0 & 0.0 & 1.06 & 0.0 & 0.89 & 0.0 & 8.66 & 8.47 \\
\hline$D_{4}$ & 9.77 & 9.03 & 6.07 & 0.0 & 0.0 & 1.07 & 0.0 & 0.89 & 0.0 & 8.67 & 8.48 \\
\hline$D_{5}$ & 2.72 & 2.47 & 7.88 & 5.27 & 6.15 & 0.0 & 5.73 & 5.17 & 5.73 & 8.46 & 2.59 \\
\hline$D_{6}$ & 9.77 & 9.03 & 6.07 & 0.0 & 0.0 & 1.06 & 0.0 & 0.89 & 0.0 & 8.67 & 8.47 \\
\hline$D_{7}$ & 9.64 & 8.97 & 7.73 & 4.79 & 5.58 & 5.2 & 5.21 & 0.0 & 5.2 & 8.51 & 8.35 \\
\hline$D_{8}$ & 9.77 & 9.03 & 6.07 & 0.0 & 0.0 & 1.06 & 0.0 & 0.89 & 0.0 & 8.67 & 8.47 \\
\hline$D_{9}$ & 10.2 & 9.57 & 0.0 & 8.72 & 10.08 & 8.69 & 9.45 & 8.11 & 9.44 & 0.0 & 0.97 \\
\hline$D_{10}$ & 3.2 & 2.96 & 5.44 & 8.24 & 9.59 & 2.89 & 8.96 & 7.62 & 8.95 & 5.14 & 0.0 \\
\hline \hline
\end{tabular}

Table 2.4: Kullback-Liebler divergences between the topic distributions from Table 2.3 Note that it is not symmetric.

Table 2.4 shows the KL divergences between the topic distributions of the example documents. As can be seen in the distances between some pairs of documents (e.g. D2D0, D5-D3, D6-D2), KL presents two major problems: (1) when a topic distribution is zero, KL divergence is not defined and (2) it is not symmetric, which does not fit well with semantic similarity measures that are usually expected to be symmetric $(\overline{R u s}$ et al., 2013). The lack of symmetry is especially remarkable between some document pairs (e.g. D10-D9, D8-D5) due to their unbalanced inter-topic distribution.

Jensen-Shannon (JS) divergence ( $\operatorname{Lin}, 1991$; Rao, 1982) solves the problems of KL considering the average of the distributions as below (Celikyilmaz et al., 2010):

$$
J S(P, Q)=\sum_{i=1}^{K} p_{i} * \log \frac{2 * p_{i}}{p_{i}+q_{i}}+\sum_{i=1}^{K} q_{i} * \log \frac{2 * q_{i}}{q_{i}+p_{i}}
$$

It can be transformed into a similarity measure as follows (Dagan et al., 1999) :

$$
\operatorname{sim}_{J S}\left(D_{i}, D_{j}\right)=10^{-J S(p, q)}
$$


where $D_{i}, D_{j}$ are the documents and $p, q$ the topic distributions of each of them.

Table 2.5 shows the JS divergences between the topic distributions of the example documents. Now the measurements are symmetrical.

\begin{tabular}{cccccccccccc}
\hline & $D_{0}$ & $D_{1}$ & $D_{2}$ & $D_{3}$ & $D_{4}$ & $D_{5}$ & $D_{6}$ & $D_{7}$ & $D_{8}$ & $D_{9}$ & $D_{1} 0$ \\
\hline \hline$D_{0}$ & 0.0 & 0.0 & 0.69 & 0.69 & 0.69 & 0.14 & 0.69 & 0.69 & 0.69 & 0.69 & 0.15 \\
\hline$D_{1}$ & 0.0 & 0.0 & 0.69 & 0.69 & 0.69 & 0.14 & 0.69 & 0.69 & 0.69 & 0.69 & 0.15 \\
\hline$D_{2}$ & 0.69 & 0.69 & 0.0 & 0.68 & 0.68 & 0.69 & 0.68 & 0.68 & 0.68 & 0.0 & 0.29 \\
\hline$D_{3}$ & 0.69 & 0.69 & 0.68 & 0.0 & 0.0 & 0.31 & 0.0 & 0.27 & 0.0 & 0.69 & 0.69 \\
\hline$D_{4}$ & 0.69 & 0.69 & 0.68 & 0.0 & 0.0 & 0.31 & 0.0 & 0.27 & 0.0 & 0.69 & 0.69 \\
\hline$D_{5}$ & 0.14 & 0.14 & 0.69 & 0.31 & 0.31 & 0.0 & 0.31 & 0.43 & 0.31 & 0.69 & 0.25 \\
\hline$D_{6}$ & 0.69 & 0.69 & 0.68 & 0.0 & 0.0 & 0.31 & 0.0 & 0.27 & 0.0 & 0.69 & 0.69 \\
\hline$D_{7}$ & 0.69 & 0.69 & 0.68 & 0.27 & 0.27 & 0.43 & 0.27 & 0.0 & 0.27 & 0.69 & 0.69 \\
\hline$D_{8}$ & 0.69 & 0.69 & 0.68 & 0.0 & 0.0 & 0.31 & 0.0 & 0.27 & 0.0 & 0.69 & 0.69 \\
\hline$D_{9}$ & 0.69 & 0.69 & 0.0 & 0.69 & 0.69 & 0.69 & 0.69 & 0.69 & 0.69 & 0.0 & 0.29 \\
\hline$D_{10}$ & 0.15 & 0.15 & 0.29 & 0.69 & 0.69 & 0.25 & 0.69 & 0.69 & 0.69 & 0.29 & 0.0 \\
\hline \hline
\end{tabular}

Table 2.5: Jensen-Shannon divergences between the topic distributions from Table 2.3

However, these metrics are not well-defined distance metrics, that is, they do not satisfy triangle inequality (Charikar, 2002). This inequality considers $d(x, z)<=$ $d(x, y)+d(y, z)$ for a metric $d$ (Griffiths et al., 2007) and places strong constraints on distance measures and on the locations of points in a space given a set of distances. As a metric axiom, the triangle inequality must be satisfied in order to take advantage of the inferences that can be deduced from it. Thus, if similarity is assumed to be a monotonically decreasing function of distance, this inequality avoids the calculation of all pairs of similarities by considering that if $x$ is similar to $y$ and $y$ is similar to $z$, then $x$ must be similar to $z$.

Let's see how the triangular inequality is present in our example. The distance between documents $D_{0}$ and $D_{6}$, for example, should be lower than or equal to the sum of the distances between $D_{0}$ and $D_{5}$, and between $D_{5}$ and $D_{6}$. KL considers $d\left(D_{0}, D_{6}\right)=9.73$ and $d\left(D_{0}, D_{5}\right)+d\left(D_{5}, D_{6}\right)=0.42+5.73=6.15$, so the triangle inequality is not satisfied since 9.73 is greater than 6.15 . The same applies to JS. 
In this case $d\left(D_{0}, D_{6}\right)=0.69$ is not lower than or equal to $d\left(D_{0}, D_{5}\right)+d\left(D_{5}, D_{6}\right)=$ $0.14+0.31=0.44$.

Hellinger (He) distance (Basseville, 1989, DasGupta, 2011) is a symmetric measure that satisfies the triangle inequality and, along with JS divergence, has been used in various fields where a comparison between two probability distributions is required (Blei and Lafferty, 2007, Boyd-Graber and Resnik, 2010, Hall et al., 2008):

$$
H e(P, Q)=\frac{1}{\sqrt{2}} \cdot \sqrt{\left.\sum_{i=1}^{K}\left(\sqrt{p_{i}}-\sqrt{q_{i}}\right)^{2}\right)}
$$

It can be transformed into a similarity measure by subtracting it from 1 (Rus et al., 2013) such that a zero distance means max. similarity score and vice versa:

$$
\operatorname{sim}_{H e}\left(D_{i}, D_{j}\right)=1-H e(p, q)
$$

\begin{tabular}{cccccccccccc}
\hline & $D_{0}$ & $D_{1}$ & $D_{2}$ & $D_{3}$ & $D_{4}$ & $D_{5}$ & $D_{6}$ & $D_{7}$ & $D_{8}$ & $D_{9}$ & $D_{1} 0$ \\
\hline \hline$D_{0}$ & 0.0 & 0.0 & 0.99 & 0.99 & 0.99 & 0.43 & 0.99 & 0.99 & 0.99 & 0.99 & 0.46 \\
\hline$D_{1}$ & 0.0 & 0.0 & 0.99 & 0.99 & 0.99 & 0.43 & 0.99 & 0.99 & 0.99 & 0.99 & 0.45 \\
\hline$D_{2}$ & 0.99 & 0.99 & 0.0 & 0.97 & 0.97 & 0.98 & 0.97 & 0.97 & 0.97 & 0.02 & 0.62 \\
\hline$D_{3}$ & 0.99 & 0.99 & 0.97 & 0.0 & 0.01 & 0.64 & 0.0 & 0.59 & 0.0 & 0.99 & 0.98 \\
\hline$D_{4}$ & 0.99 & 0.99 & 0.97 & 0.01 & 0.0 & 0.64 & 0.0 & 0.6 & 0.0 & 0.99 & 0.99 \\
\hline$D_{5}$ & 0.43 & 0.43 & 0.98 & 0.64 & 0.64 & 0.0 & 0.64 & 0.78 & 0.64 & 0.99 & 0.59 \\
\hline$D_{6}$ & 0.99 & 0.99 & 0.97 & 0.0 & 0.0 & 0.64 & 0.0 & 0.6 & 0.0 & 0.99 & 0.99 \\
\hline$D_{7}$ & 0.99 & 0.99 & 0.97 & 0.59 & 0.6 & 0.78 & 0.6 & 0.0 & 0.6 & 0.98 & 0.98 \\
\hline$D_{8}$ & 0.99 & 0.99 & 0.97 & 0.0 & 0.0 & 0.64 & 0.0 & 0.6 & 0.0 & 0.99 & 0.99 \\
\hline$D_{9}$ & 0.99 & 0.99 & 0.02 & 0.99 & 0.99 & 0.99 & 0.99 & 0.98 & 0.99 & 0.0 & 0.61 \\
\hline$D_{10}$ & 0.46 & 0.45 & 0.62 & 0.98 & 0.99 & 0.59 & 0.99 & 0.98 & 0.99 & 0.61 & 0.0 \\
\hline \hline
\end{tabular}

Table 2.6: Hellinger distances between the topic distributions from Table 2.3

Table 2.6 shows the Hellinger distances between the topic distributions of the example documents. Now the triangular inequality is satisfied and can be verified by seeing that $d\left(D_{0}, D_{6}\right)=0.99$ is lower than $d\left(D_{0}, D_{5}\right)+d\left(D_{5}, D_{6}\right)=0.43+0.64=1.07$. 
In order to make the $J S$ divergence satisfy the triangular inequality, (Endres and Schindelin, 2003) proposed S2JSD as a distance metric based on the square root of two times the $J S$ divergence:

$$
\operatorname{S2JSD}(P, Q)=\sqrt{2 * J S(P, Q)}
$$

Table 2.7 shows the S2JSD distances between the topic distributions of the example documents. As before, the triangular inequality is satisfied and can be verified by seeing that $d\left(D_{0}, D_{6}\right)=1.18$ is lower than $d\left(D_{0}, D_{5}\right)+d\left(D_{5}, D_{6}\right)=0.52+0.79=1.31$.

\begin{tabular}{cccccccccccc}
\hline & $D_{0}$ & $D_{1}$ & $D_{2}$ & $D_{3}$ & $D_{4}$ & $D_{5}$ & $D_{6}$ & $D_{7}$ & $D_{8}$ & $D_{9}$ & $D_{10}$ \\
\hline \hline$D_{0}$ & 0.0 & 0.0 & 1.18 & 1.18 & 1.18 & 0.52 & 1.18 & 1.18 & 1.18 & 1.18 & 0.55 \\
\hline$D_{1}$ & 0.0 & 0.0 & 1.18 & 1.18 & 1.18 & 0.52 & 1.18 & 1.18 & 1.18 & 1.18 & 0.55 \\
\hline$D_{2}$ & 1.18 & 1.18 & 0.0 & 1.17 & 1.17 & 1.17 & 1.17 & 1.17 & 1.17 & 0.03 & 0.76 \\
\hline$D_{3}$ & 1.18 & 1.18 & 1.17 & 0.0 & 0.01 & 0.79 & 0.01 & 0.73 & 0.01 & 1.18 & 1.18 \\
\hline$D_{4}$ & 1.18 & 1.18 & 1.17 & 0.01 & 0.0 & 0.79 & 0.0 & 0.73 & 0.0 & 1.18 & 1.18 \\
\hline$D_{5}$ & 0.52 & 0.52 & 1.17 & 0.79 & 0.79 & 0.0 & 0.79 & 0.93 & 0.79 & 1.18 & 0.71 \\
\hline$D_{6}$ & 1.18 & 1.18 & 1.17 & 0.01 & 0.0 & 0.79 & 0.0 & 0.73 & 0.0 & 1.18 & 1.18 \\
\hline$D_{7}$ & 1.18 & 1.18 & 1.17 & 0.73 & 0.73 & 0.93 & 0.73 & 0.0 & 0.73 & 1.18 & 1.17 \\
\hline$D_{8}$ & 1.18 & 1.18 & 1.17 & 0.01 & 0.0 & 0.79 & 0.0 & 0.73 & 0.0 & 1.18 & 1.18 \\
\hline$D_{9}$ & 1.18 & 1.18 & 0.03 & 1.18 & 1.18 & 1.18 & 1.18 & 1.18 & 1.18 & 0.0 & 0.76 \\
\hline$D_{10}$ & 0.55 & 0.55 & 0.76 & 1.18 & 1.18 & 0.71 & 1.18 & 1.17 & 1.18 & 0.76 & 0.0 \\
\hline \hline
\end{tabular}

Table 2.7: S2JSD distances between the topic distributions from Table 2.3 .

\subsection{Research Areas in Topic Modelling Techniques}

This thesis aims to enable a semantic-aware exploration of the knowledge arising from large and multilingual document collections by exploiting the capabilities of topic models and their metric spaces. There are several research areas of ongoing related work.

The first one is topic creation and reuse, key for understanding the steps needed to transform the unstructured data from a text into numerical values based on prob- 
abilistic topics. The way in which topic models are created and reused is crucial to addressing large-scale analysis.

The second area is topic explainability, which refers to the capacity of topics to capture and describe the content of a text. Topic explainability is important for making understandable the relationships that are derived from the inference of topic distributions.

The third area is document similarity, where the ability to measure the semantic difference between texts from the distance between their topic distributions is addressed.

Finally, the fourth area is multilingual topics, as we aim to explore collections of texts written in different languages through their topic-based relationships. A strategy to relate the topics of each language is proposed.

These four areas are closely related to each other. Having efficient thematic representations of texts, distance metrics based on shared themes, and mechanisms to abstract the particularities of a language to represent the themes, may help to organize large multilingual document collections.

Each area and its limitations are described below. A summary can be found in Table 2.8 .

\subsubsection{Topic Creation and Reuse}

Textual content usually includes non-relevant information. Keeping only what can bring value for the involved agents (general consumers, experts, companies, investors...) becomes a challenge. A necessary first step before leveraging documents for knowledgeintensive tasks is to preprocess them following different techniques. Recent studies (Westergaard et al., 2017) have shown, in the scientific domain, that mining full-text articles gives consistently better results than only using sections or summaries. Given the size limitations and concise nature of summaries, they often omit descriptions or results that are considered to be less relevant but still important for some IR tasks (Divoli et al., 2012). Since this behavior is present in multiple domains, our interest is focused on processing full texts, not only summaries or parts of texts, as we will show in the remainder of this thesis.

There is a broad set of algorithms able to analyze text for producing annotations at very different levels of granularity: from minimal units such as terms and entities, 


\begin{tabular}{|c|c|c|}
\hline Area & Scope & Limitation \\
\hline topic creation and reuse & $\begin{array}{l}\text { process texts, train topic } \\
\text { models and calculate topic } \\
\text { distributions from large } \\
\text { corpora }\end{array}$ & $\begin{array}{l}\text { horizontal scalability (i.e. } \\
\text { distributed computing) } \\
\text { generally ignored in favor } \\
\text { of vertical scalability } \\
\text { (i.e. more computational } \\
\text { power). No unified or } \\
\text { standardized models for } \\
\text { exchanging topic models }\end{array}$ \\
\hline topic explainability & $\begin{array}{l}\text { describe and relate docu- } \\
\text { ments by topics }\end{array}$ & $\begin{array}{l}\text { high dimensional models } \\
\text { makes them difficult to in- } \\
\text { terpret }\end{array}$ \\
\hline document similarity & $\begin{array}{l}\text { compare topic distribu- } \\
\text { tions by measuring their } \\
\text { distances }\end{array}$ & $\begin{array}{l}\text { unaffordable complexity in } \\
\text { huge collections }\end{array}$ \\
\hline multilingual topics & $\begin{array}{l}\text { topic distributions across } \\
\text { languages }\end{array}$ & $\begin{array}{l}\text { parallel or comparable } \\
\text { training data required }\end{array}$ \\
\hline
\end{tabular}

Table 2.8: Research areas and limitations. 
to descriptors at the level of the entire collection, such as summaries or topics. This includes methods and tools to perform Part-of-Speech (PoS) tagging, to recognize Named Entities (NER), or to create topic models following LDA or any other approach (e.g. LSA or pLSA). But their implementations have been designed to work in an isolated, non-collaborative way (Agerri et al., 2014; Manning et al., 2014). They have not paid special attention to facilitating their interoperability and they use closed data formats that increase the technological dependence and limits the reuse possibilities. For example, a topic model created by Mallet ${ }^{19}$ can only make inferences if it is used from Mallet itself or using its libraries, since there is no unified or standardized format for distributing topic models. In that example, the fact that Mallet is implemented in Java makes it difficult to reuse their models from other programming languages (e.g. Python).

Some recent approaches focus on the creation and exploitation of topic models through an API based on libraries ${ }^{20}$ or web services ${ }^{21}$ (Lisena et al., 2020). However they are focused on the operations that can be performed on the model rather than abstracting the topic model as a resource. Others provide loca $\mathbb{2 2}^{22}$ or remote $\notin^{23}$ ecosystems to create and reuse learning models (e.g. Hugging Face ${ }^{24}$ ), but their data format is not open and cannot be (re)used out of the environment. To the best of our knowledge, existing efforts registered in the literature do not propose a unified model to exchange topic models, understood as an already accepted standards-based format. In this thesis we propose reusable topic models and a scalable framework to create and use them.

\subsubsection{Topic Explainability}

Even though the distance metrics mentioned in Section 2.2 have been proposed and used in the SoA, making sense out of the similarity score based on comparing topic distributions is not easy. As shown in figures 2.3 and 2.4 , given a set of pairs of documents, their similarity scores vary according to the number of topics used to represent documents. So the distances between the same pairs fluctuate from being more to less

\footnotetext{
${ }^{19}$ http://mallet.cs.umass.edu

20 https://bab2min.github.io/tomotopy

21 https://github.com/D2KLab/ToModAPI

22 https://onnx.ai/

23 https://vespa.ai

${ }^{24}$ https : //huggingface.co/
} 
distant when changing the number of topics, and are hence difficult to use to relate documents semantically in an understandable and consistent manner. Understanding which of those distances is better for representing the underlying collection is a challenge.

Distances between documents based on topic distributions generally increase as the number of dimensions of the space increases, because it is a vector space. This is due to the fact that as the number of topics describing the model increases, the more specific the topics will be. Topics shared by a pair of documents can be broken down into more specific topics that are not shared by those documents. Document similarity is then dependent on the model used to represent documents when considering this type of metrics.

We know that absolute distances between documents vary when we tune $\alpha$ and $\beta$ hyper-parameters differently, but we also see that "relative distances" also change. Imagine that we have three documents, A, B and C, described by a topic model, M1. The distance from the topic distribution of A to B is less than from A to C. However, in a second topic model, M2, trained with the same documents than M1 but with different hyper-parameters, the distance from the topic distribution of $\mathrm{A}$ to $\mathrm{C}$ is less than to B (cross-lines in figures 2.3 and 2.4). This behaviour highlights the difficulty of establishing absolute similarity thresholds and the complexity to measure distances taking into account all dimensions of a topic model. A pair of documents may be similar when they are represented in low-dimensional topic models, and not similar when high-dimensional models are used to represent them. Distance thresholds should be model-dependent rather than general, and metrics flexible enough to handle dimensional changes. In this thesis we go beyond the thematic and lowdimensional feature space created by topic models and propose a hierarchical feature space suitable for big real-world data sets, where documents are only described by their most relevant topics.

\subsubsection{Document Similarity}

In addition, document similarity comparisons are too costly to be performed in huge collections of data and require more efficient approaches than having to calculate all pairwise similarities. Using a naive approach creating a similarity matrix with all document comparisons takes $O\left(n^{2}\right)$ time (where $n$ is the number of documents), so obtaining 


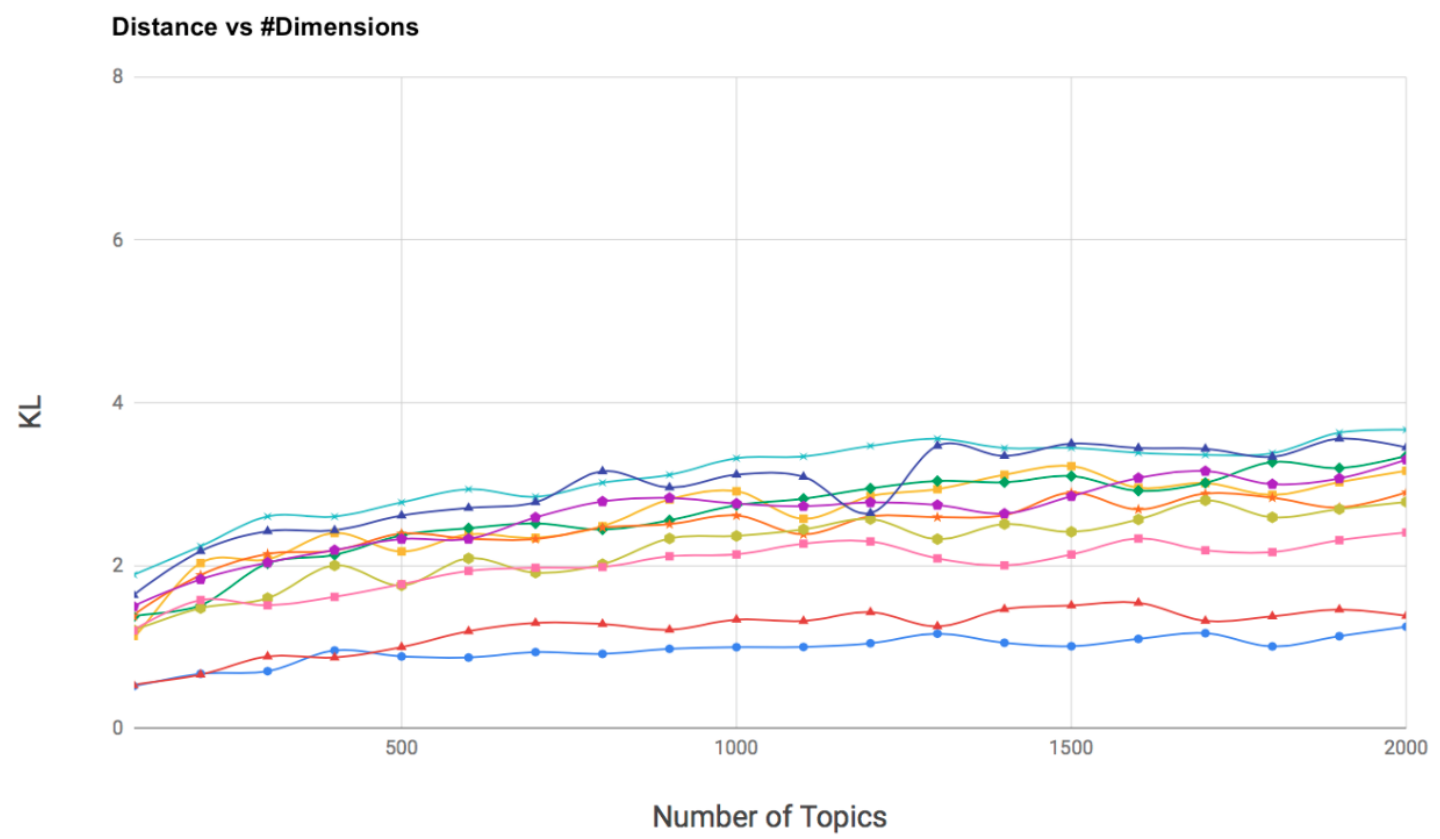

(a) Kullback-Liebler divergence

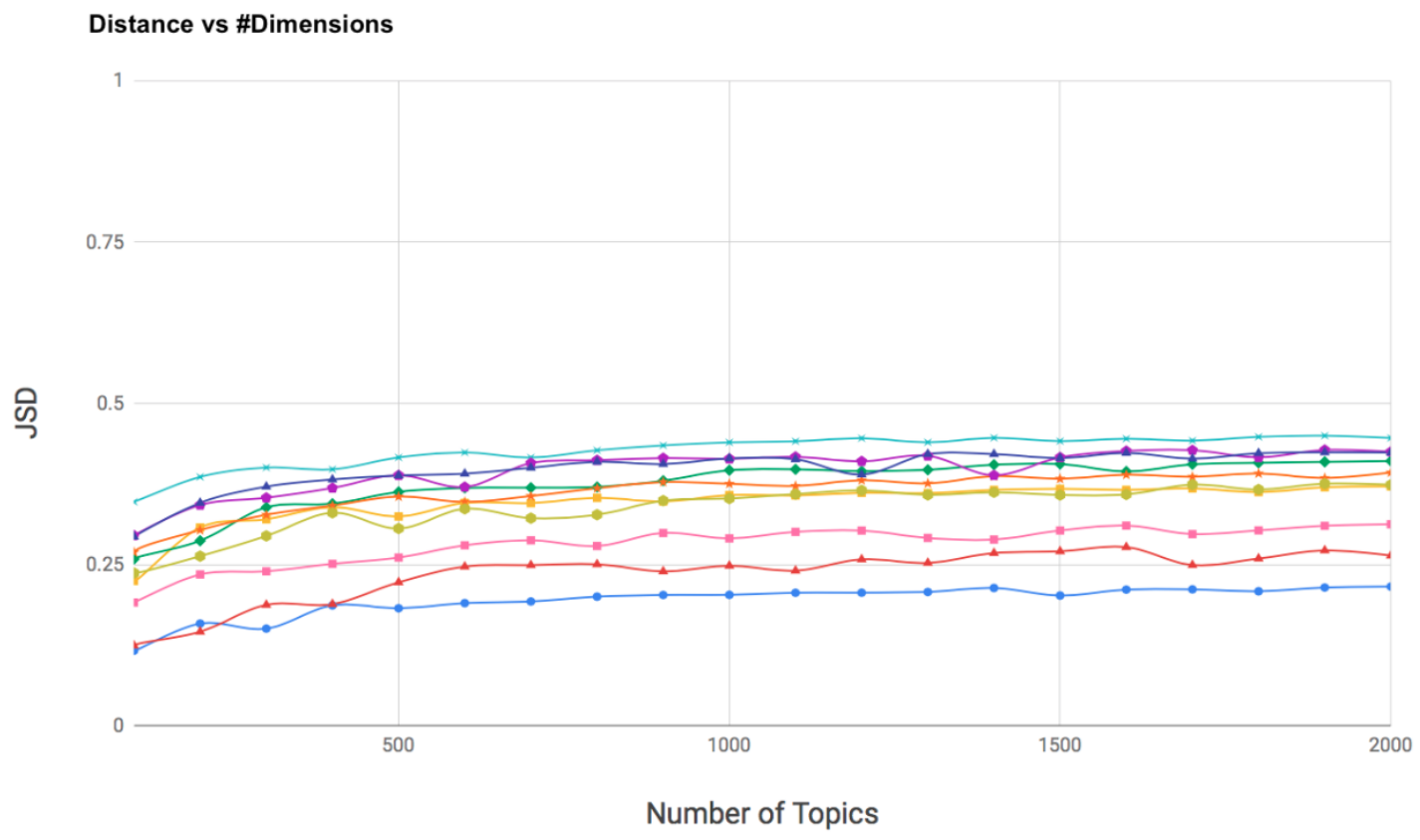

(b) Jensen-Shannon divergence

Figure 2.3: Evolution of the distances based on KL (a) and JS (b) metrics between a set of document pairs when increasing the number of topics in the models (Badenes-Olmedo et al., 2020b). 


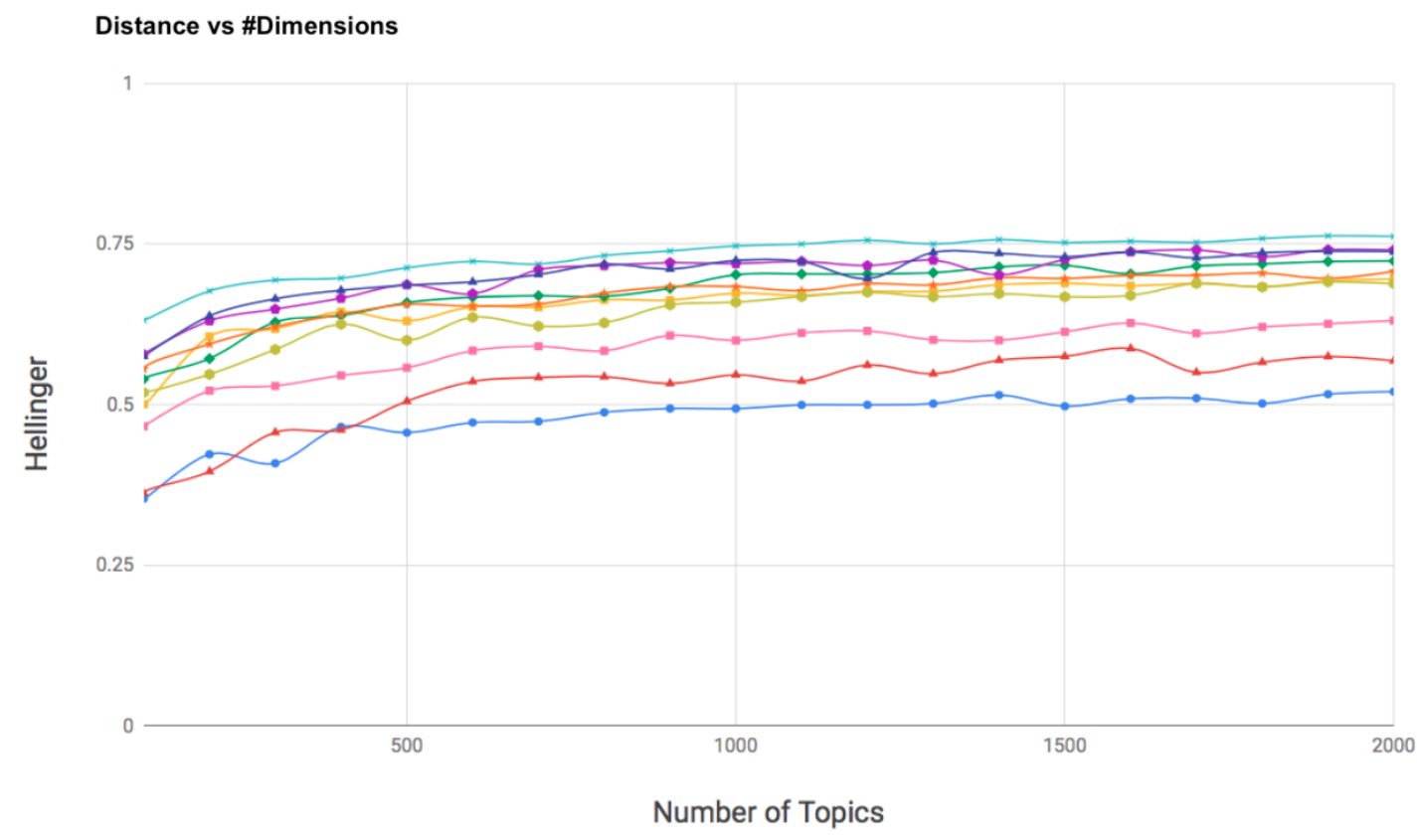

(a) Hellinger distance

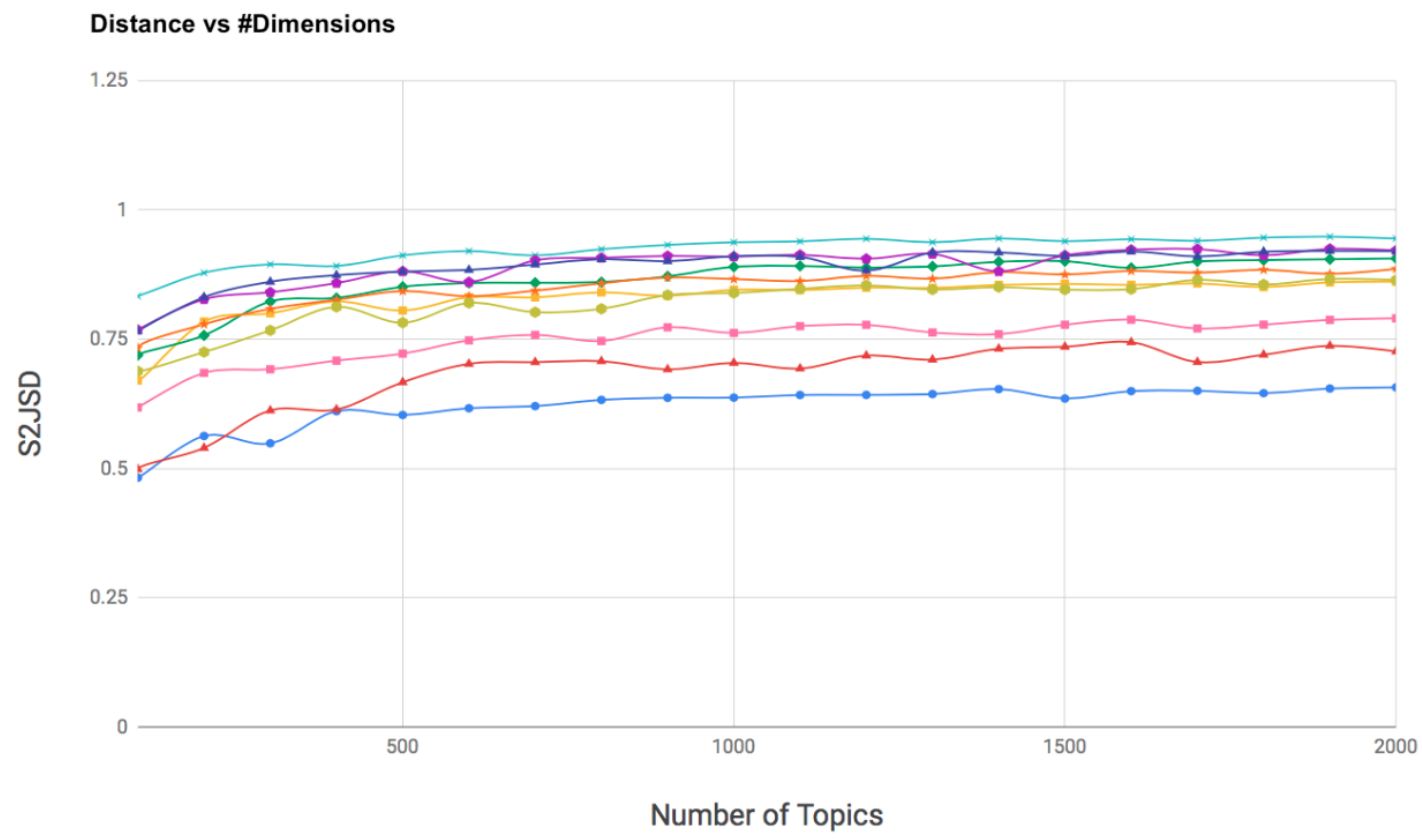

(b) S2JSD distance

Figure 2.4: Evolution of the distances based on He (a) and S2JSD (b) metrics between a set of document pairs when increasing the number of topics in the models (Badenes-Olmedo et al. 2020b). 
all possible pairs of similarities in a large collection of documents (e.g. a corpus of 32 million patents) can be unfeasible because of the quadratic cost of comparing every pair of elements. Many different approaches have been proposed to reduce this complexity. For instance, computation can be approximated by a nearest neighbors (ANN) search problem (Indyk and Motwani, 1998). ANN search is an optimization problem that finds nearest neighbors of a given query in a metric space of $n$ points.

Due to the low storage cost and fast retrieval speed, hashing is one of the most popular solutions for ANN search (Zhen et al., 2016). This technique transforms data points from the original feature space into a binary-code space, so that similar data points have larger probability of collision (i.e. having the same hash code). This type of formulation for the document similarity comparison problem has proven to yield good results in the metric space (Krstovski and Smith, 2011) due to the fact that ANN search has been designed to handle distance metrics (e.g. cosine, Euclidean, Manhattan). But distance metrics between topic distributions should be informationtheoretically motivated metrics (e.g. Hellinger, Kullback-Leibler divergence, JensenShannon divergence) since they compare density functions.

These challenges can be tackled by hashing methods based on clusters of topics to measure similarity, instead of directly using their weights. Hashing methods transform the data points from the original feature space into a binary-code Hamming space, where the similarities in the original space are preserved. They can learn hash functions (data-dependent) or use projections (data-independent) from the training data (Wang et al., 2016). Data-independent methods, unlike data-dependent ones do not need to be re-calculated when data changes, i.e. adding or removing documents to the collection. Taking large-scale scenarios into account (e.g. Document clustering, Content-based Recommendation, Duplicate Detection), data independency is a key feature along with the ability to infer hash codes individually (for each document) rather than on a set of documents. Data-independent hashing methods depend on two key elements: (1) data type and (2) distance metric. For vector-type data, as introduced in section 2.2 , based on $l_{p}$ distance with $p \epsilon[0,2)$ lots of hashing methods have been proposed, such as p-stable Locality-Sensitive Hashing (LSH) (Datar et al., 2004), Leech lattice LSH (Andoni and Indyk, 2006), Spherical LSH (Terasawa and Tanaka, 2007), and Beyond LSH (Andoni et al., 2014). Based on the $\theta$ distance many methods have been developed such as Kernel LSH (Kulis and Grauman, 2012) and Hyperplane hashing 
(Vijayanarasimhan et al., 2014). But only few methods handle density metrics in a simplex space, where topic distributions are projected. A first approach transformed the $H e$ divergence into an Euclidean distance so that existing ANN techniques, such as LSH and k-d tree, could be applied (Krstovski et al., 2013). But this solution does not consider the special attributions of probability distributions, such as Non-negative and Sum-equal-one. Recently, a hashing schema (Mao et al., 2017) has been proposed taking into account the symmetry, non-negativity and triangle inequality features of the S2JSD metric for probability distributions. For set-type data, Jaccard Coefficient is the main metric used. Some examples are K-min Sketch (Li et al., 2012), Min-max hash (Ji et al., 2013), B-bit minwise hashing (Li and König, 2010) and Sim-min-hash (Zhao et al., 2013).

All of these approaches have demonstrated efficiency in the search for similar documents, but none of them considers the search for documents (1) by thematic areas or (2) by similarity levels, nor they offer (3) an explanation about the similarity obtained beyond the vectors used to calculate it. In addition, binary-hash codes drop a very precious information: the topic relevance. This thesis proposes a hash function-based approach that allows efficiently searching for related documents while maintaining topic-based annotation, preserving the notion of why two documents are related.

\subsubsection{Multilingual Topic Alignment}

When the IR task is also cross-language, document retrieval must be independent of the language of the user's query. At execution time, the query in the source language is typically translated into a target language with the help of a dictionary or a machinetranslation system. But for many languages we may not have access to translation dictionaries or a full translation system, or they can be expensive to execute, or expensive to train (lot of data required) in an online search system. In such situations it is useful to rely on smaller annotation units derived from the text so the full content does not need to be translated, for instance by finding correspondences with regard to the topics that are present in both the query and the documents being searched.

Some methods use document-aligned corpora, where documents are grouped and constrained to the same topic distribution during training to align the different languages (Fukumasu et al., 2012; Mimno et al., 2009; Ni et al., 2009; Zhang et al., 2013), or 
theme-aligned corpora, where similar themes and ideas appear in all languages $\overline{\text { Boyd- }}$ Graber and Blei, 2009). Multilingual Probabilistic Topic Models (MuPTM) (Vulić et al., 2015) emerged in this area as a group of language-independent generative machine learning models that can be used on theme-aligned multilingual texts. They are based on LDA, adding supervised associations between languages by using parallel corpora, with sentence-aligned documents (e.g. Europar 25 corpus), or comparable corpora, with theme-aligned documents (e.g. Wikipedia ${ }^{26}$ articles), in multiple languages. Once a MuPTM has been generated, documents can be represented by data points in a single feature space based on topics to detect similarities among them exploiting inference results and using distance metrics. Due to its generic language-independent nature and the power of inference on unseen documents, MuPTM's have found many interesting applications in many different cross-lingual tasks. They have been used on cross-lingual event clustering (De Smet and Moens, 2009), document classification (De Smet et al., 2011; Ni et al., 2011), semantic similarity of words (Mimno et al., 2009; Vulić and Moens, 2012), information retrieval (Ganguly et al., 2012; Vulić and Moens, 2013), document matching (Platt et al., 2010; Zhu et al., 2013), and others.

There are also methods based on word alignments from bilingual dictionaries instead of aligned corpora. Topic models emerge as distributions over crosslingual equivalence classes of words (Hao and Paul, 2018; Jagarlamudi and Daumé, 2010; Shi et al., 2016; Zhang et al. 2010). Others propose to translate only the words used to characterize the topics across the languages, such as anchor words (Yuan et al., 2018) or top words (Yang et al. 2019). A recent approach is placed between word and document alignments since it proposes crosslingual topic models using the language-independent categories assigned to each Wikipedia article (Piccardi and West, 2020). Instead of using bags-ofwords to represent texts, which would be language dependent, it explores the references of each article and represents them through bags-of-links, using the categories of each reference to represent the texts.

However, the requirement of parallel/comparable corpora or dictionaries limits the usage of these models in many cross-lingual situations. There are not many document collections that can be used for training since large parallel corpora are rare in most of the use cases, especially for languages with fewer resources.

\footnotetext{
${ }^{25}$ https://ec.europa.eu/jrc/en/language-technologies/dcep

${ }^{26}$ https://www.wikipedia.org/
} 


\begin{tabular}{cl}
\hline \multicolumn{1}{c}{ Area } & \multicolumn{1}{c}{ Proposal } \\
\hline \hline topic creation and reuse & $\begin{array}{l}\text { distributed framework to create and (re)use } \\
\text { topic models }\end{array}$ \\
\hline topic explainability & hierarchical feature space suitable for big \\
& real-world data sets, where documents are \\
& only described by their most relevant topics \\
\hline document similarity & hash function-based algorithm that allows \\
& searching for related documents efficiently \\
& while maintaining topic-based annotation, \\
& preserving the notion of why two documents \\
& are related \\
\hline multilingual topics & cross-lingual topics based on hierarchical \\
& representations of concepts to browse \\
& multi-lingual document collections without \\
& the need for parallel or comparable corpora \\
\hline
\end{tabular}

Table 2.9: Research areas and proposals. 
Moreover, in order to incorporate new languages or update the existing associations, these models must be (re)trained with documents from all languages at the same time, making it difficult to scale to large corpora (Hao et al., 2018; Moritz and Büchler, 2017). Inspired by the work of (Boyd-Graber and Resnik, 2010), where the use of concepts to align topics from different languages is proposed, we take MuPTM a step further by making it cross-lingual through representations based on topic hierarchies. We do not only use conceptual representations of the top words in a topic, but we group the words hierarchically by relevance and compare their conceptual representations at those levels. Documents are represented by topics described with language-independent concepts and multilingual corpora can efficiently be browsed without the need for translation. In this thesis we propose to automatically infer cross-lingual topics to browse multilingual document collections without the need for parallel or comparable corpora.

A summary with the proposals of this thesis to overcome the limitations of each research area (described in table 2.8) can be found in table 2.9. 


\section{Chapter 3}

\section{Methodology}

The work presented in this thesis aims to facilitate the exploration of huge collections of multilingual documents through thematic associations inferred from their content. It assumes that, given a multilingual corpus, related documents will contain a similar distribution of topics. Each of the challenges arising from this objective defines a working dimension and guides the research carried out in this thesis.

The first dimension focuses on efficiency, in order to create the text processing flows that are required to create or apply probabilistic topic learning models. The workload required to process a corpus varies according to the number of documents, the length of texts and the kind of knowledge (annotations) that need to be inferred from the text. If the design of the workflow is scalable, there is no need to modify the processing logic when working with larger collections of documents, since adding a reasonable amount of computational resources is enough to perform it. These resources can be machines (i.e horizontal scaling) or processing units (e.g CPU, RAM) in an existing machine (i.e vertical scaling).

The second dimension covers the explainability of the text annotations when projected into spaces where they are manipulated for diverse tasks. The idea behind these spaces is to represent documents as points (or vectors in a vector space) that are close together when the texts are semantically related, and far apart when they are semantically distant. The ability of these spaces to create meaningful representations is also studied in this work.

In the third dimension, we explore the complexity of data structures to organize texts from their representations based on probabilistic topics. Divisions of space into 
semantically-related regions are convenient to allow browsing large document collections. The representativeness covered in the previous dimension enables the interpretation of the relations and regions obtained.

And finally, the fourth dimension handles the multilingualism of collections that contain documents in several languages. On a multilingual space, documents are described and related across languages.

This chapter introduces our main hypothesis (section 3.1), and the associated research challenges (section 3.2), and presents the research methodology (section 3.3).

\subsection{Research Hypotheses}

We define our main hypothesis as follows:

Hypothesis 1 Large multilingual document collections can be automatically analyzed to discover thematic representations that enable an exploration through related texts.

Our hypothesis can be divided into four different sub-parts, which are related to the aforementioned efficiency, explainability, complexity, and multilinguality dimensions. First, by distributing across different computation nodes both natural language processing tasks and topic models we can efficiently process huge collections of documents (H1.1).

Second, it is possible to semantically relate documents by comparing their most relevant topics (H1.2). Furthermore, for this purpose we hypothesize that the use of topic hierarchies (H1.2.1) and similarity metrics based on relevance levels (H1.2.2) help quantifying the semantic distance between texts. Third, by dividing the representational space into regions based on topics and relevance levels we can search for related documents without having to calculate all pairwise comparisons and without discarding the notion of topics for further processing $(\boldsymbol{H 1 . 3})$.

And finally, it is possible to relate documents in different languages without having to translate them, by using language agnostic concepts from their main topics (H1.4).

A summary of the hypotheses and how they tackle our research dimensions can be found in Table 3.1 . 


\begin{tabular}{|c|c|}
\hline Hypothesis & Research Dimension \\
\hline $\begin{array}{l}\text { H1: Large multilingual document collections } \\
\text { can be automatically analyzed to discover the- } \\
\text { matic representations that enable an explo- } \\
\text { ration through related texts }\end{array}$ & $\begin{array}{l}\text { D1: Efficiency, D2: Ex- } \\
\text { plainability, D3: Complex- } \\
\text { ity, D4: Multilinguality }\end{array}$ \\
\hline $\begin{array}{l}\text { H1.1: it is possible to efficiently annotate doc- } \\
\text { uments on a large scale by distributing across } \\
\text { different computation nodes natural language } \\
\text { processing tasks and topic models }\end{array}$ & D1: Efficiency \\
\hline $\begin{array}{l}\text { H1.2: it is possible to semantically relate texts } \\
\text { from their most relevant topics }\end{array}$ & D2: Explainability \\
\hline $\begin{array}{l}\text { H1.3: it is possible to find relevant documents } \\
\text { with similar topic distributions without cal- } \\
\text { culating all pairwise comparisons and without } \\
\text { discarding the notion of topics from their rep- } \\
\text { resentation }\end{array}$ & D3: Complexity \\
\hline $\begin{array}{l}\text { H1.4: it is possible to relate documents in } \\
\text { different languages without having to trans- } \\
\text { late them, by using language agnostic concepts } \\
\text { from their main topics }\end{array}$ & D4: Multilinguality \\
\hline
\end{tabular}

Table 3.1: Hypotheses and research dimensions. 


\subsection{Research Challenges}

Several research challenges emerge from these hypotheses. First, in order to facilitate reusing existing topic models by processing systems with different architectures and technological stacks, we need to define topic-model programming interfaces. Second, in order to describe and thematically relate documents, we must address how to produce explainable topic-based associations. Third, by working with huge collections of documents described by topics, we need to handle large-scale comparisons of topic distributions. Finally, in order to explore multilingual document collections from shared topic-based representational spaces, we have to provide automatic cross-lingual topic alignment. Each of these research challenges are described below and covered throughout this thesis.

\subsubsection{Scalable Creation and Inference of Topics}

Although some initiatives to facilitate the reuse of machine-learning models exist in the literature as discussed in section 2.4.1, there are still some restrictions that limit a wider use of topic models across programming languages and Tech infrastructures. Technical dependencies or closed data formats are the main reasons that prevent or make reproducibility of these models difficult by imposing relevant restrictions to work with them. Normally, only the inference capacity of the models is exploited, and some of their internal properties, such as the weights of the words in each topic or the distance between topics, are not enabled which prevents it from being used for a different purpose than when it was created. Reuse of topic models is limited by incompatibility problems (RCInterface1).

The properties and functions offered by a topic model vary according to the method used to build it. Some methods (e.g. Mallet) hide the weights of words in each topic and limits the topic representation to the first $n$ words. Others (e.g. Gensim) creates topic models that allow inferring topic distribution of texts and words, but does not measure the distance between topics. The ability to reproduce or to evaluate the work done in this area and to reuse topic models without losing information or knowledge is then limited. There is no standard to specify the attributes and operations that a topic model can provide (RCInterface2). Sometimes topics are described by the top ten or five most relevant words, and occasionally these word lists are not accompanied by weights, 
making a density-based analysis impossible. These differences in presenting the models can sometimes limit their reusability if they cannot infer new topic distributions even when the learning algorithm allows for it.

\subsubsection{Explainable Topic-based Relations}

In order to facilitate the exploration of document collections, vector space models are often used to semantically relate texts based on their word distributions. As described in Section 2.2, these models first create a dictionary with the words used in the collection, and then represent documents by vectors whose dimensions correspond to each word in the dictionary. In large collections, these models need to be adapted to make operations on vectors more manageable. As a result, topic models are a new abstraction method that reduces the dimensions of vectors. Topics are described by word distributions over the entire vocabulary and documents by vectors containing topic distributions. Despite the extensive use of these representation models, there is no common criteria for identifying the most representative topics in a document (RCExplainable1).

In addition, since similarity metrics over this representation space are based on accumulating the difference in topic densities, it is difficult to explain the distance between topic distributions (RCExplainable2). And, unless a minimum distance threshold can be defined or a set of n-top topics agreed, there is no common criterion for determining whether two documents are related (RCExplainable3).

\subsubsection{Large-scale Comparisons of Topic Distributions}

There are many scenarios where we need to find related documents (e.g. a researcher doing literature review, or an R\&D manager analyzing project proposals). Experts can benefit from discovering those connections to achieve these goals, but brute-force pairwise comparisons are not computationally adequate when the size of the corpus is too large. Some algorithms in the literature divide the search space into regions containing potentially related documents, which are later processed separately from the rest in order to reduce the number of pairs compared. However, there are no mechanisms that efficiently partition the topic-based search space without compromising the ability for thematic exploration (RCComparison1).

In addition, documents from the same region should be compared and there are no similarity metrics that compare partial distributions of topics (RCComparison2). 


\subsubsection{Unsupervised Cross-lingual Topic Alignment}

With the ongoing growth in the number of texts in different languages, we need annotation methods that enable browsing multilingual corpora. As discussed in section 2. multilingual probabilistic topic models have recently emerged as a group of semisupervised machine learning models that can be used to perform thematic explorations on collections of texts in multiple languages. However, there are no approaches that $a b$ stract the representation of probabilistic topics in language-independent spaces without translating texts or aligning documents (RCCrossLingual1). Existing approaches require parallel or comparable training data to create a language-independent space.

A summary of the challenges covered in this work and how they map to the hypotheses is presented in table 3.2 .

\subsection{Research Methodology}

The research presented in this thesis is based on four dimensions or research areas as discussed in section 3.2. Each one is motivated by different research problems that we need to solve in order to achieve our ultimate goal of making it easier to explore large multilingual document collections through their topics. Once a dimension is tackled, the next one is considered, and so on. This iterative and incremental methodology allows refining the research results by evaluating them with more experiments and addressing increasingly complex research problems.

Figure 3.1 shows the dimensions on which the research of this thesis has been built. The top of the pyramid is only reached once the lower dimensions are dealt with successfully. They are presented as a chain of four steps. The first step describes the motivation to perform a given task coming from real-world problems that we had to deal with, and is represented by a brown arrow. In the context of this task, the research problem arises and is framed by a pink arrow. For each of them a solution is proposed and evaluated according to a specific criterion. The proposed solution is represented by a green arrow and the evaluation with a blue arrow. Once a proposal has been validated, the next dimension of the pyramid is achievable and all the previous research problems are added to the new research problem as conditions to be taken into account. 


\begin{tabular}{|c|c|}
\hline Research Challenge & Hypotheses \\
\hline $\begin{array}{l}\text { RCInterface1: reuse of topic models is lim- } \\
\text { ited by incompatibility problems }\end{array}$ & $\begin{array}{l}\text { H1.1: documents can be efficiently annotated } \\
\text { on a large scale by distributing across dif- } \\
\text { ferent computation nodes both natural lan- } \\
\text { guage processing tasks and topic models }\end{array}$ \\
\hline $\begin{array}{l}\text { RCInterface2: there is no standard that uni- } \\
\text { fies the representation of topic models }\end{array}$ & $\begin{array}{l}\text { H1.1: documents can be efficiently annotated } \\
\text { on a large scale by distributing across dif- } \\
\text { ferent computation nodes both natural lan- } \\
\text { guage processing tasks and topic models }\end{array}$ \\
\hline $\begin{array}{l}\text { RCExplainable1: there is no common criteria } \\
\text { for identifying the most representative topics } \\
\text { in a document }\end{array}$ & $\begin{array}{l}\text { H1.2: texts can be semantically related from } \\
\text { their most relevant topics, H1.3: documents } \\
\text { with similar topic distributions can be found } \\
\text { without calculate all pairwise comparisons } \\
\text { and without losing the ability to explore } \\
\text { them through their topics }\end{array}$ \\
\hline $\begin{array}{l}\text { RCExplainable2: it is difficult to understand } \\
\text { the distance between topic distributions }\end{array}$ & $\begin{array}{l}\text { H1.2: texts can be semantically related from } \\
\text { their most relevant topics }\end{array}$ \\
\hline $\begin{array}{l}\text { RCExplainable3: there is no common crite- } \\
\text { rion for determining whether documents are } \\
\text { related }\end{array}$ & $\begin{array}{l}\text { H1.2: texts can be semantically related from } \\
\text { their most relevant topics }\end{array}$ \\
\hline $\begin{array}{l}\text { RCComparison1: there are no mechanisms } \\
\text { that efficiently partition the topic-based } \\
\text { search space without compromising the abil- } \\
\text { ity for thematic exploration }\end{array}$ & $\begin{array}{l}\text { H1.3: documents with similar topic distribu- } \\
\text { tions can be found without calculate all pair- } \\
\text { wise comparisons }\end{array}$ \\
\hline $\begin{array}{l}\text { RCComparison2: there are no similarity } \\
\text { metrics that compare partial distributions of } \\
\text { topics }\end{array}$ & $\begin{array}{l}\text { H1.3: documents with similar topic distribu- } \\
\text { tions can be found without calculate all pair- } \\
\text { wise comparisons }\end{array}$ \\
\hline $\begin{array}{l}\text { RCCrossLingual1: there are no approaches } \\
\text { to abstract probabilistic topics in language- } \\
\text { independent spaces without translating texts } \\
\text { or aligning documents }\end{array}$ & $\begin{array}{l}\text { H1.4: documents in different languages can } \\
\text { be related without having to translate them } \\
\text { using language agnostic concepts from their } \\
\text { main topics }\end{array}$ \\
\hline
\end{tabular}

Table 3.2: Open Research Challenges and Hypotheses. 


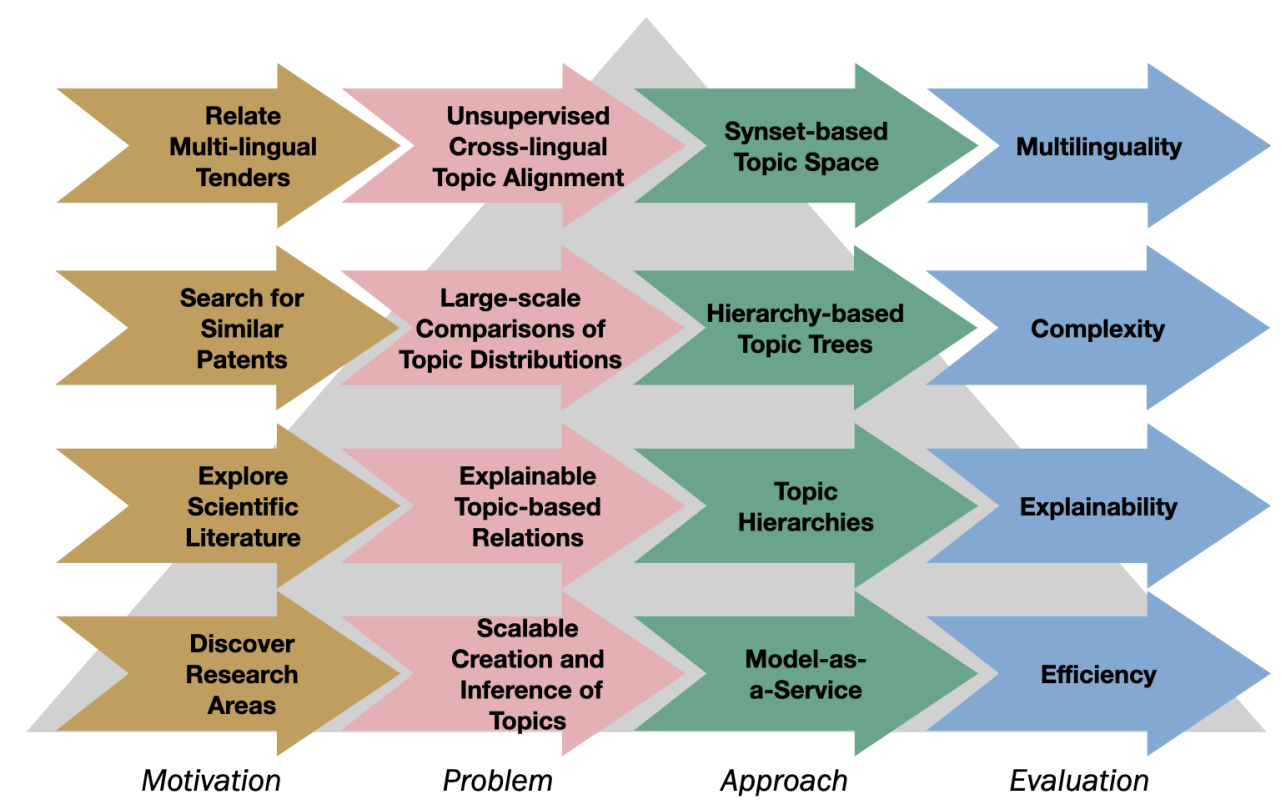

Figure 3.1: Research dimensions of the thesis. The first ones must be overcome before reaching higher dimensions.

Technical objectives (i.e., develop a new resource) or research objectives (i.e., discover the solution to a problem) guide the solution proposal before moving on to the next dimension. They are presented below, organized by the research problem associated with each dimension.

- Scalable Creation and Inference of Topics: This first dimension arose when we had to analyze a huge collection of documents describing research and innovation projects to discover which research areas are being addressed, measure their presence in the collection, and characterize them so their presence can be inferred in unseen documents. Such a high volume of data made difficult to process it manually, so we needed to automate the required processing to draw insights from it. Probabilistic topics allow describing research areas, so we defined a distributed text-processing model for creating large probabilistic topic models (RO1) and a Web service template to distribute them (RO2). In this way, the models themselves could be easily integrated into scalable text processing pipelines. As a result, we created a scalable platform for topic modeling (TO1), and produced a model-as-a-service repository with pre-trained topic models (TO2). The efficiency 
of this solution was validated by processing a corpus of 100,000 documents collected from the CORDIS dataset ${ }^{27}$, which contains descriptions of projects funded by the European Union under a framework programme since 1990 (BadenesOlmedo et al., 2017b).

The main contributions under this dimension are described in Chapter 4 as follows:

- a software architecture to process big volumes of textual documents in a distributed and decoupled manner;

- the definition of a model-as-a-service template for probabilistic topic models;

- an implementation of the architecture, librAIry, following those design principles.

- Explainable Topic-based Relations: In the second dimension we needed to browse scientific papers through their content-based relations. The problem of massively annotating documents with topic distributions came up. We had to create annotations based on topic models in a way that was computationally affordable and enabled a semantic-aware exploration of the knowledge inside them (RO3). Once documents were annotated, a metric that compares documents and facilitates their interpretation from topic annotations (RO4) was required. As a result, we integrated the annotation method into the topic model service (TO3) and implemented a text comparison metric based on partial representations of topics. These proposals were validated by classifying 500,000 scientific articles from the Open Research Corpu 28 in domains such as Computer Science, Neuroscience and Biomedicine (Badenes-Olmedo et al., 2017a, 2019a, 2017c).

The main contributions under this dimension are described in Chapter 5 as follows:

- a clustering algorithm based on probabilistic topic distributions;

- a hash function to transform topic distributions into topic hierarchies;

- a similarity metric based on topic sets.

${ }^{27}$ https://data.europa.eu/euodp/es/data/dataset/cordisH2020projects

${ }^{28}$ https://allenai.org/data/open-research-corpus 
- Large-scale Comparisons of Topic Distributions: This dimension covered the search for related documents based on their most relevant topics. Thanks to having dealt with the above two dimensions, large collections of documents could be annotated with topic hierarchies and text distances could be measured from their annotations. Now, the aim was to find related documents without losing the exploratory capacity offered by topics. Similarity comparisons were too costly to be performed in such huge collections of data and required more efficient approaches than having to calculate all pairwise similarities. We applied techniques based on approximate nearest-neighbors to organize documents in regions with similar topic hierarchies (RO5). As a result, we developed a system to automatically find related documents ( $\mathbf{T O} 4$ ). It was validated on a collection of one million texts retrieved from the United States patents corpus 29 . The relations between patents derived from their manual categorization were compared with those automatically obtained from their topic distributions (Badenes-Olmedo et al., 2019a, 2020b).

The main contributions under this dimension are described in Chapter 6 as follows:

- a data structure to partition the search space and organize documents described by topic hierarchies;

- a corpus browser that leverages these representations to automatically relate documents.

- Unsupervised Cross-lingual Topic Alignment: Finally, a new dimension on top of the previous ones emerged to relate texts coming from different languages. In particular, since document relations were based on their topics, this dimension was focused on aligning topics without supervision from models trained with texts in different languages. Since each language defined its own vocabulary, the topics were model-specific and could not be directly compared. We abstracted the topic representations to create a single space out of the particularities of the language (RO6). This approach was validated on the English, Spanish, French, Italian and Portuguese editions of the JCR-Acquis 30 corpora and revealed promising

\footnotetext{
${ }^{20}$ https://www.uspto.gov/ip-policy/economic-research/research-datasets

30 https://ec.europa.eu/jrc/en/language-technologies/jrc-acquis
} 


\begin{tabular}{|c|c|}
\hline Research Objective & Research Challenge \\
\hline $\begin{array}{l}\text { RO1: Define a distributed text-processing model } \\
\text { for creating large probabilistic topic models }\end{array}$ & RCInterface1 \\
\hline $\begin{array}{l}\text { RO2: Define a template to package probabilistic } \\
\text { topic models as web services }\end{array}$ & RCInterface2 \\
\hline $\begin{array}{l}\text { RO3: Define annotations based on topics that en- } \\
\text { able a semantic-aware exploration of the knowledge } \\
\text { inside a corpus }\end{array}$ & RCExplainable1 \\
\hline $\begin{array}{l}\text { RO4: Define a metric based on topic annotations } \\
\text { that compares documents and facilitates their in- } \\
\text { terpretation }\end{array}$ & $\begin{array}{l}\text { RCExplainable2, RCExplain- } \\
\text { able3 }\end{array}$ \\
\hline $\begin{array}{l}\text { RO5: Define nearest-neighbor techniques to orga- } \\
\text { nize documents in regions with similar topic hierar- } \\
\text { chies }\end{array}$ & $\begin{array}{l}\text { RCComparison1, RCCompari- } \\
\text { son2 }\end{array}$ \\
\hline $\begin{array}{l}\text { RO6: Define a transformation of the topic-based } \\
\text { annotations to create a unique representational } \\
\text { space out of the particularities from each language }\end{array}$ & RCCrossLingual1 \\
\hline TO1: Create a scalable platform for topic modeling & RCInterface1, RCInterface2 \\
\hline $\begin{array}{l}\text { TO2: Create a repository of Topic-based web ser- } \\
\text { vices }\end{array}$ & RCInterface2 \\
\hline $\begin{array}{l}\text { TO3: Integrate the annotation method based on } \\
\text { topic hierarchies into the topic model service }\end{array}$ & $\begin{array}{l}\text { RCExplainable2, RCCompari- } \\
\text { son2 }\end{array}$ \\
\hline $\begin{array}{l}\text { TO4: Create a system capable of finding related } \\
\text { documents automatically }\end{array}$ & $\begin{array}{l}\text { RCExplainable2, RCExplain- } \\
\text { able3, RCComparison1, RC- } \\
\text { CrossLingual1 }\end{array}$ \\
\hline
\end{tabular}

Table 3.3: Research and technical objectives and their related challenges. 
results on classifying and sorting documents by related content across languages (Badenes-Olmedo et al., 2019a b).

The main contributions under this dimension are described in Chapter 7, as follows:

- an algorithm to represent probabilistic topics using concept sets;

- a repository of aligned topic models from the English, Spanish, French, Italian and Portuguese editions of the JRC-Acquis corpus.

Table 3.3 summarizes the research objectives (ROs), technical objectives (TOs) and connects them with the research challenges (RCs) from Table 3.2 . 


\section{Chapter 4}

\section{Creation and Distribution of Probabilistic Topic Models}

This chapter addresses the research hypothesis H1.1 (documents can be efficiently annotated on a large scale by distributing across different computation nodes both natural language processing tasks and topic models), the research objectives RO1 (define a distributed text-processing model for creating large probabilistic topic models) and RO2 (define a template to package probabilistic topic model as web services), and the technical objectives TO1 (create a scalable platform for topic modeling) and TO2 (create a repository of topic-based services). It presents librAIry (Badenes-Olmedo et al., 2017b), our framework to exploit probabilistic topic models through a service-oriented approach. In doing so, we reuse existing techniques and web standards to create online services, which aim to make our results reusable and interoperable with other alternative approaches.

\subsection{Topic Modeling Framework}

As discussed in Section 2.4, topic models have been successfully used in multiple domains (Greene and Cross, 2016; He et al., 2017; Nzali et al., 2017; O'Neill et al., 2017). Each domain and use case has different characteristics that need to be considered when constructing, exposing and exploiting probabilistic topic models. Some with longer texts, others with shorter texts, some with millions of documents, others with only a few hundred or thousands, some have only one computing unit to process them, others 


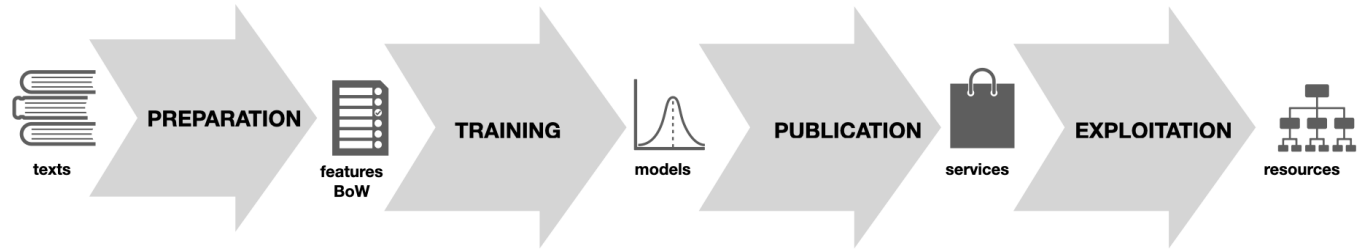

Figure 4.1: Stages in the creation and reuse of topic models. Texts are first processed to retrieve tokens and create bags-of-words (BoW). These structures are used to train a model that identifies word distributions called topics. The model is enabled to make topic inferences in unseen texts. It is published as a web service in an online repository. The service can then be (re)used as web resource, for example to categorize documents.

have multiple nodes distributed among several distributed machines. Adapting to such diversity, topic modeling algorithms have evolved since their inception in (Deerwester et al. 1990) to be more efficient in challenging situations (Liu et al., 2015b). However, the methods reported in papers are mostly focused on the learning process and a topic model life-cycle is broader than just the creation of the model (Fig. 4.1). It covers a first stage of document preparation, where texts are divided first into phrases and then into words that are normalized before being counted to create bags-of-words (BoW). The next stage (training stage), seeks patterns among word distributions and as a result a topic model is created. The model is then packaged for distribution (publication stage). And finally the model can be used and reused (exploitation stage). In order to have a framework that covers the entire process of creating, publishing, using and reusing probabilistic topic models in both large- and small-scale contexts, we have focused on adapting techniques and standards widely used in the software engineering domain. In this section we cover the first two stages of the life-cycle: text preparation and model training. In Section 4.2.1 we describe how the models are published, and in section 4.2 .2 how they can be exploited.

librAIry is a framework that covers the entire topic model life-cycle by combining learning algorithms with natural language processing methods and software distribution techniques. The main objective is to facilitate the creation of reusable probabilistic topic models by minimizing their technical dependencies. Methods and algorithms proposed in this thesis have been implemented and evaluated in this framework, which therefore serves as the technological basis for our research. 
Our design requirements, which have guided our development process, can be organized into three categories:

- Corpora representation requirements, which tackle the modeling of document collections and its metadata. This includes texts and their related annotations.

- Task distribution requirements, which refer to event management to notify changes in document collections. Coordination of this information is crucial for robust and reproducible results.

- Process execution requirements, which capture the operations involved in creating a topic model. The parallel task execution leads to the creation of models.

The rest of the section describes how we have adapted existing techniques and standards in librAIry to address each of the requirement categories described above. An open, distributed and scalable framework has been developed whose source code is publicly available for reuse ${ }^{31}$ and which is registered in the Intellectual Property Office of Comunidad de Madrid with reference M-7342-2016.

\subsubsection{Corpora Representation for Topic Modeling}

Inspired by a Staged Event-Driven Architecture (SEDA) that exchanges messages and handles status changes, our framework is based on resources and actions. A resource can be a document that represents raw texts (e.g. a full-text research paper), or a snippet of text with a logical part (e.g. sections, summaries or even phrases grouped by their rhetoric), or a domain that contains a dataset of texts (e.g. a conference proceedings) or even an annotation made on them (e.g. review comments, namedentities, topics). Actions can be executed on resources to change their status (e.g. create, update or delete).

To better illustrate this model, take a sample of the research articles published at the K-CAP 2019 conference ${ }^{32}$ (see Fig. 4.2). A document resource is created for each publication and contains the full text of the article. Each document is then associated

\footnotetext{
31 https://doi.org/10.5281/zenodo.4561156

32 http://www.k-cap.org/2019
} 


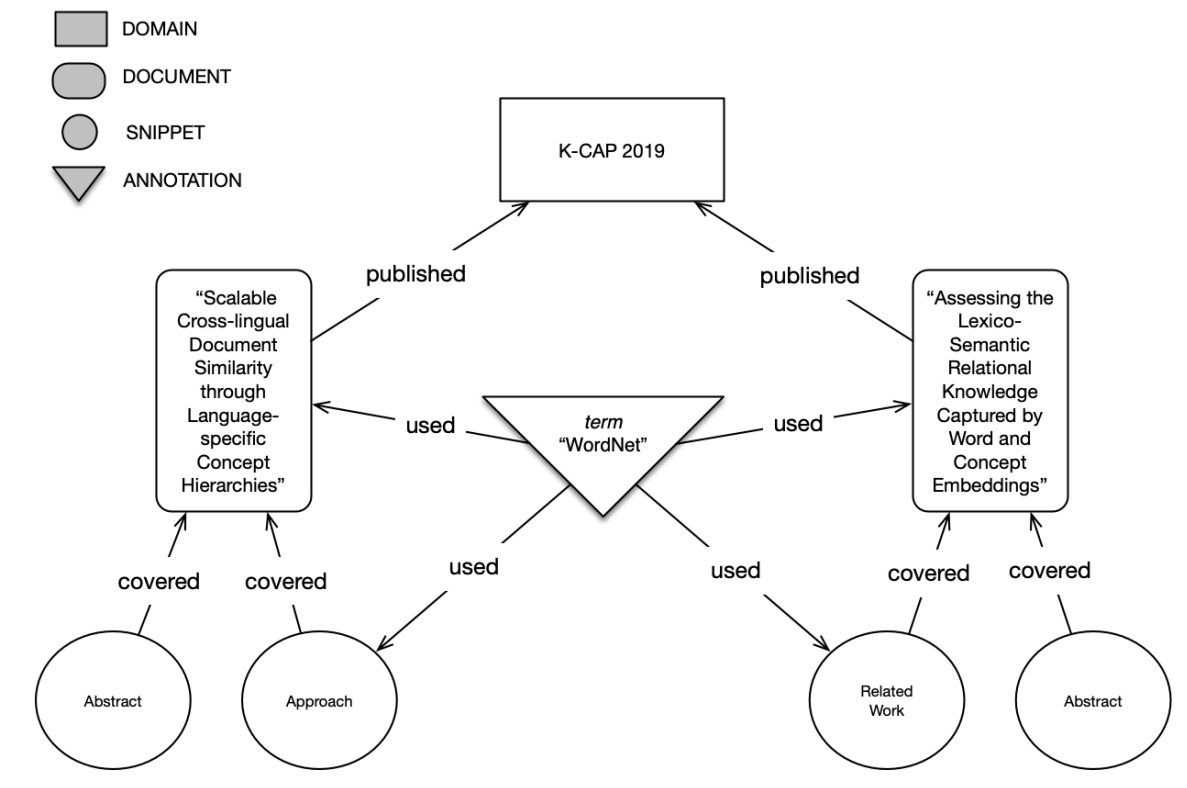

Figure 4.2: Representation of two scientific papers published at the International Conference on Knowledge Capture (K-CAP, 2019) that mention the same entity, Wordnet, in different sections.

with several snippets, one for each section of the article (e.g. abstract, introduction, conclusions, etc). Finally, a domain is created that groups all these documents under the same conference. This initial representation can be extended with annotations, that can provide more detailed information at different levels (e.g. named-entities, keywords or topics).

Resources and actions are individually addressable and linkable (Turchi et al., 2012) following Linked Data principles (Bizer et al., 2009). Each of them has: (1) a name, (2) a retrievable (or dereferenceable) HTTP URI so that it can be looked up, (3) a descriptive information provided by using standard notation (e.g. JavaScript Object Notation (JSON)) when it is looked up by URI, and (4) links to other URIs so that other resources can be discovered from it.

More details about each of them is shown below. 


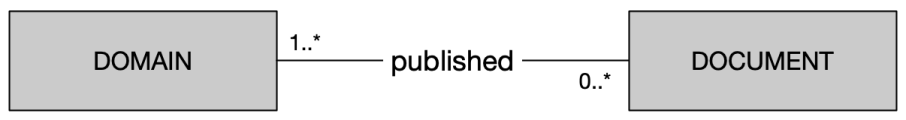

Figure 4.3: Relation between domain and document.

\subsubsection{Domain}

A domain is an aggregation of documents. It is described as a group with parts separately described. Every document that is processed belongs, at least, to one domain (Fig 4.3).

A domain contains the following information:

- uri: identifier created from the resource type (i.e domains) and a Universally Unique Identifer (UUID) (e.g domains/88b86fa6-11c8-11eb-adc1-0242ac120002)

- creation-time: date when resource was created. It follows the ISO-8601 133 ,

- name: label associated to the resource.

- description: additional information about it.

\subsubsection{Document}

A document is a resource consisting primarily of text. Examples include research papers, articles, books or patents. It follows the Open Archives Initiative for Object Reuse and Exchang $\AA^{34}$ (OAI-ORE) and the Dublin Core Metadata Initiativ $\AA^{35}$ (DCMI).

A document contains the following information:

- uri: identifier created from the resource type (i.e documents) and a UUID ( e.g documents/809af686-11c8-11eb-adc1-0242ac120002)

- creation-time: date when resource was created. It follows the ISO-8601.

- publishedOn: date when resource was published. It follows the ISO-8601.

\footnotetext{
$3_{3}^{3}$ https://www.iso.org/standard/40874.html

${ }^{34}$ http://www . openarchives.org

35 http://dublincore.org
} 
- publishedBy: an entity responsible for making the document available. It can be a person, an organization or a service. It may be different from the entity that conceptually formed the resource (e.g. wrote the document), which is recorded as authoredBy. This entity should be identified by a valid Uniform Resource Identificator (URI) such as WebId $\sqrt{36}$, orcid $\sqrt{37}$ or internal URI.

- authoredOn: the time the document was conceptually formed. The author time should be present if different from publishedOn. It must be a formatted timestamp following ISO-8601.

- authoredBy: an entity primarily responsible for making the content of the document. It may be a list to indicate multiple authors. Each of them identified by a valid URI such as WebId, orcid or internal URI.

- retrievedFrom: a URI identifying the repository or source from which the document was derived. This property should be accompanied with retrievedOn.

- retrievedOn: the time the document was retrieved on. If this property is present, the retrievedFrom must also be present. It must be a formatted timestamp following ISO-8601.

- format: the physical or digital manifestation of the resource. Typically, it includes the media-type (i.e the IANA code ${ }^{38}$ of the document.

- language: the language(s) in which the document was written. It is defined by RFC-1766 39 with a two-letter language code followed, optionally, by a two-letter country code.

- title: a name given to the document. It is a name by which the document is formally known.

- description: it may include but is not limited to an abstract, or a free-text account of the content.

- rights: information about rights helds in and over the document.

\footnotetext{
${ }^{36}$ http://www.w3.org/wiki/WebID

${ }^{37}$ http://orcid.org

${ }^{38}$ http://www.iana.org

39 http://www.ietf.org/rfc/rfc1766.txt
} 


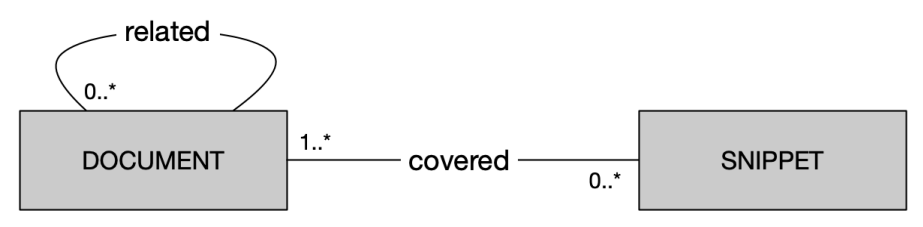

Figure 4.4: Relation between document and snippet.

- content: raw text from the document.

Furthermore, a document may contain zero or more snippets and a snippet may belong to one or more documents. Since librAIry can also discover relations among documents, a document may contain zero or more references to other documents (Fig. 4.4).

\subsubsection{Snippet}

A snippet is a resource that is included either physically or logically in a document. In a scientific document, for example, it may be the abstract section or a logical set of sentences sharing the same rhethorical class (e.g. approach, background, related-work, etc). As seen above (Fig. 4.4, a snippet can belong to one or more documents.

It contains the following information:

- uri: identifier created from the resource type (i.e snippets) and a UUID ( e.g snippets/7a5a46c8-11c8-11eb-adc1-0242ac120002)

- creation-time: date when resource was created. It follows the ISO-8601.

- sense: content-type. It refers to a section or any other criteria under which the following text makes sense (e.g. introduction, summary, notes, etc).

- content: partial text retrieved from the full-text of a document.

\subsubsection{Annotation}

Annotations are data retrieved from resources that can be used to relate them. They are basically key-value data structures associated to domains, documents or snippets. Examples are entities mentioned in a text, or topics covered in a collection. Any 


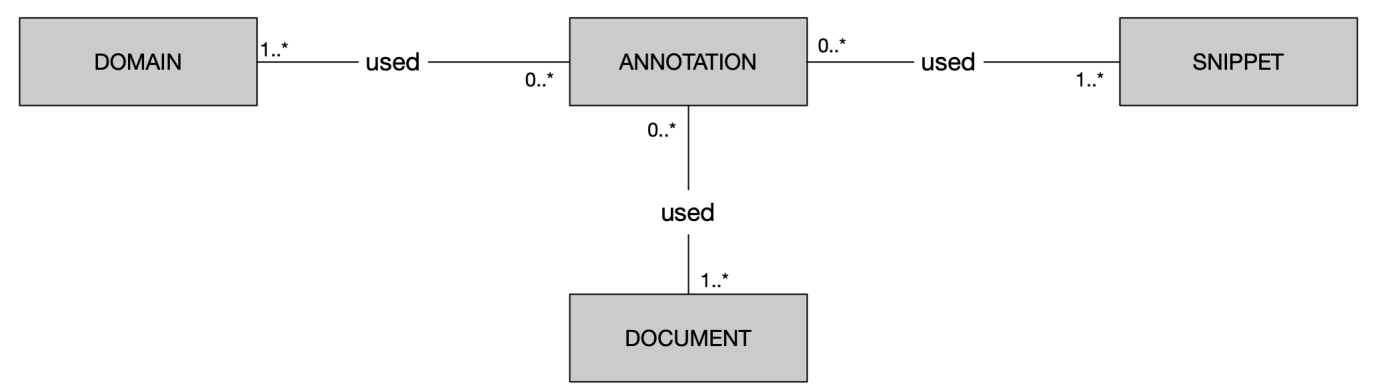

Figure 4.5: Relation between annotations and other resources.

resource can have zero or multiple annotations, which can be shared among several resources (Fig. 4.5)

It contains the following information:

- uri: identifier created from the resource type (i.e annotations) and a UUID ( e.g annotations/73671e68-11c8-11eb-adc1-0242ac120002)

- creation-time: date when resource was created. It follows the ISO-8601.

- key: a category or type associated with the information it contains (e.g. entity, comment, topic, keywords, etc). Recommended best practice is to use a controlled vocabulary.

- value: a note about the resource in the form of free text.

\subsubsection{Event-oriented Processing Workflow}

Along with the resources mentioned above, there are two additional elements that provide a crucial behavior to the framework: modules and events. An event is a nonpersistent time-based occurrence that describes a new action performed on a resource. Modules are responsible for carrying out operations on the resources (e.g. tokenize a document or create a topic model from a domain). Events are broadcasted so that any module is aware of the changes made to the resources and can perform actions on one or more resources in response to a new state reached by a given resource. These actions are paralleled since modules are replicated through distributed environments. 


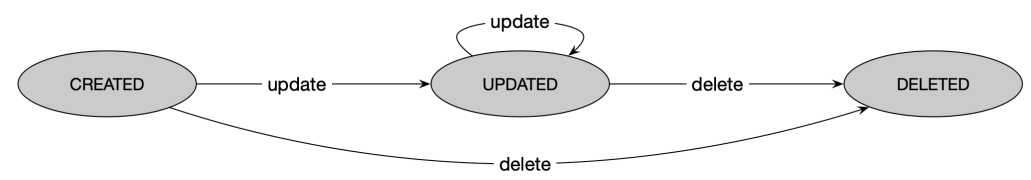

Figure 4.6: Resource states.

The framework follows a publisher/subscriber approach where modules can publish and read events to notify and to be notified about the state of a resource (Fig. 4.6). An event notifies a performed action (i.e. a resource and its new state), and follows the Representational State Transfer (REST) paradigm (Fielding and Taylor, 2002). It contains the resource type and the new state reached by a specific resource ( i.e created, deleted or updated). For example, when a new domain is created, an event message is published to the channel: domain.created. A channel is a space where events are published and modules can be subscribed to read only some events. The actions performed by a module depend on the events to which it is subscribed. Therefore, the workflow of the framework is neither static nor explicitly defined. A distributed dynamic workflow emerges according to the modules subscribed to the event channels.

From a technical point of view, librAIry uses the Advanced Message Queuing Protocol (AMQP) as the messaging standard to avoid any technical dependency to the message broker (i.e the server that sends and receives messages). This protocol defines: exchanges, queues, routing-keys and binding-keys to communicate publishers (i.e message senders) and consumers (i.e message readers). Exchanges are like message inboxes, and queues are subscribed to them by specifying the message types they are interested in with a binding-key. A message sent by a publisher to an exchange is routed with a routing-key and consumers matching that routing-key with their binding-key (used to connect the queue to that exchange), will receive the message. This mechanism allows sending and receiving messages between consumers and producers by means of shared keys (i.e. routing-keys and binding-keys). A key follows the structure: resource.status. Since a wildcard-based definition can be used to set the key, this paradigm allow modules both listening to individual type events (e.g. domains.created for new domains), or multiple type events (e.g. \#.created for all new resources). 


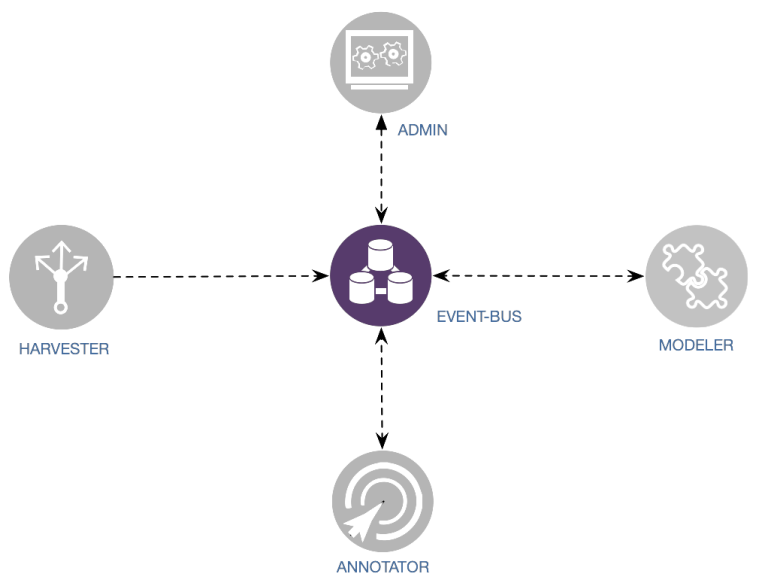

Figure 4.7: Modules (in gray) publishing or receiving events from the messenger service (in purple).

\subsubsection{Module-based Model Training}

A microservice-oriented style has been used to define the framework architecture. Using multiple services, the system analyzes texts, creates probabilistic topic models, publishes them as new services and uses them to annotate texts. A service is equivalent to a functionality, and each functionality is materialized by a module in the system. A module is then a cohesive and independent process (Dragoni et al., 2016) with a specific purpose (i.e functionality) based on the events to which it responds. These events correspond to the routing- and binding- keys attached to the module.

There are four types of modules (Fig. 4.7):

- Harvester: creates resources such as documents, snippets and domains, from local or remote repositories with textual files. We have developed harvesters to create scientific resources from Elsevier ${ }^{40}$ or any other digital repository 41 that follows the Open Archives Initiative Protocol for Metadata Harvesting (OAI$\mathrm{PMH})_{42}^{\sqrt{2}}$ as well as more general ones to retrieve public datasets from datos.gob.e: 43 or resources located on local folder 44 .

\footnotetext{
${ }^{40}$ https://github.com/librairy/harvester-elsevier

${ }^{41}$ https://github.com/librairy/harvester-research

42 https://github.com/cbadenes/camel-oaipmh

43 https://github.com/librairy/harvester-datosGobEs

44 https://github.com/librairy/harvester
} 
- binding-queue (i.e listening for): nothing

- routing-key (i.e publishing to): document.created, snippet.created, domain.(created;updated)

- Annotator: creates annotations (e.g named-entities, bag-of-words, topic distributions, etc) in documents, snippets or domains. We have developed an NLP Annotator 45 that creates bag-of-words from a given text by normalizing its content through natural language processing tasks such as named entity recognition, lemmatization or part-of-speech (PoS) tagging. The source code ${ }^{46}$ is publicly available for reuse. Topic models are also annotators in this framework, as will be seen in section 4.2.1,

- binding-key: document.(created;updated), snippet.(created;updated)

- routing-key: annotation.(created;deleted)

- Modeler: creates probabilistic topic models for domains from the bag-of-word annotations of each document. We have developed a LDA Modeler ${ }^{47}$ as well as a $\mathrm{W} 2 \mathrm{~V}$ Modeler ${ }^{48}$ (the latter for testing purposes).

- binding-key: domain.(created;updated), annotation.created

- routing-key: annotation.(created;deleted)

- Administrator: performs user-driven tasks such as reading/writing/updating resources or database queries. As with the other modules, the source code ${ }^{49}$ is publicly available for reuse. Together with the API, we have also developed a web interface 50 to visualize documents, their relationships and the topics associated with each domain.

- binding-key: \#.\#

\footnotetext{
45 http://librairy.linkeddata.es/nlp

${ }^{46}$ https://github.com/librairy/nlp

47 https://github.com/librairy/modelerTopics-service

48 https://github.com/librairy/modeler-w2v

49 https://github.com/librairy/api

${ }^{50}$ https://github.com/librairy/explorer
} 


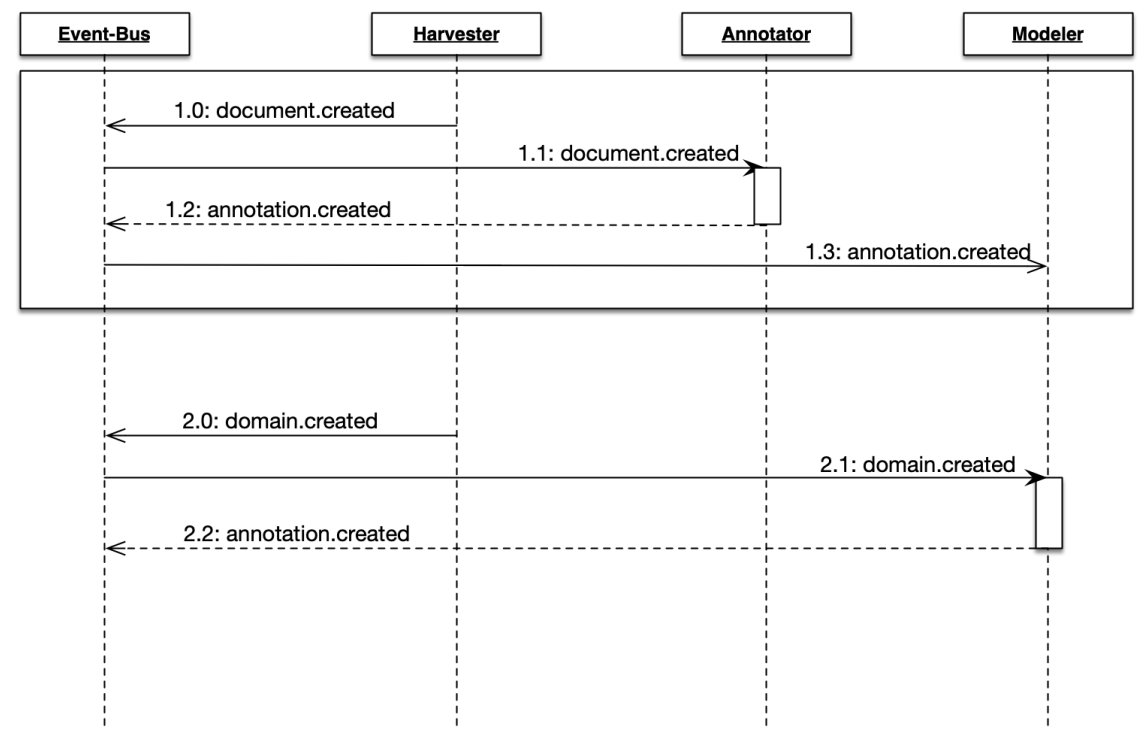

Figure 4.8: Sequence of events exchanged between modules to create a topic model from the documents added to a domain.

- routing-key: domain.(created;updated;deleted), document.(created;updated;deleted), snippet.(created;updated;deleted), annotation.(created;updated;deleted)

Figure 4.8 shows a sequence diagram that illustrates how modules work collaboratively to create a topic model from documents added to the framework. Each module provides an Application Program Interface (API) over HTTP, that follows the web standards for the RESTful API development, and a Avrd 51 based interface over TCP for efficiency reasons. Among them the communication is done over TCP.

\subsection{Topic Modeling Services}

In order to use an existing topic model in our framework, which is micro-servicesoriented, the model itself needs to be a service. This approach decouples the resources used to train a probabilistic topic model (e.g. data format or algorithm implementation), from the resources required to make inferences and thus avoids unexpected

$\sqrt[51]{\text { https://avro.apache.org }}$ 
incompatibilities. In this way, we simultaneously facilitate the reuse of topic models and also their scalable execution.

\subsubsection{Topic Model Publication}

We propose distributing topic models as services hosted in online repositories. Models are packaged as OS-level virtualization software that can run reliably applications from one computing environment to another. A model becomes a standalone and executable software package that includes everything needed to use it: code, data, runtime libraries, system tools and settings.

There are several technologies that can virtualize services. Among them, Docker ${ }^{52}$ stands out as a de facto standard due to its wide adoption. It is a platform as a service (PaaS) environment that use operating service-level virtualization to deliver software in packages called containers. Containers are isolated from one another and bundle their own software, libraries and configuration files. All containers are run by a single operating system kernel and therefore use fewer resources than virtual machines.

Topic Models in librAIry are packaged as Docker containers and published in online repositorie 53 so they can be easily downloaded and run on any machine or software solution. Containers do not only offer virtualization advantages, but also version and license control, since they handle some information that we use to characterize our models:

- repository: model name (e.g. dbpedia-model).

- author: model creator (e.g. librAIry)

- version: model version (e.g. 3.0)

- license: model license (e.g. Apache 2.0)

\subsubsection{Topic Model Exploitation}

Once a topic model has been packaged as a virtual service (i.e. Docker containers), either assembled as a annotator in librAIry or as an online service accessible via its

\footnotetext{
52 https://www.docker.com/

53 https://hub.docker.com/repositories
} 
HTTP (based on a OpenAPI interface, Fig. 4.9 or TCP (based on an Avrd 54 interface) API, it should support all tasks that can be performed on a topic model. Taking into account the reproducibility of a model, the conditions under which the model has been created, in relation to the corpus (i.e. number of documents, vocabulary size, language,.. ), about NLP tasks (i.e stopwords, PoS filtering) and about the model itself (i.e. hyperparameters) should be collected and provided by the service. Taking into account the exploration of a model, the topics and their word distributions must also be available. The word distribution is usually omitted when publishing topic models providing only the top10 or top 5 words per topic. This limits the capacity of the model to be exploited in tasks where that information is required, for example this thesis (as will be seen in chapter 4). And finally, taking into account the topic inference in new texts, which is the most extended ability of these models, the service must be able to calculate topic distributions from texts.

A summary of the tasks, and their purposes, provided by a topic model can be found in Table 4.1.

\begin{tabular}{|c|c|}
\hline Task & Purpose \\
\hline T1: Replication & create similar topics \\
\hline T2: Exploration & browse topics and words \\
\hline T3: Inference & calculate topic distributions \\
\hline
\end{tabular}

Table 4.1: Potential uses of a topic model.

In order to cover these tasks by topic services, the API must offer operations that, individually or partially, allow them to be achieved. A topic model is: reproducible (T1) when its hyperparameters and the configuration of the training set are known, explorable (T2) when the word distributions of each topic are known, and is interpretable (T3) when the presence of each topic can be measured in a text. Table 4.2 summarizes the operations offered by a topic model-as-a-service to support the potential uses.

The rest of the section describes how the model implements these operations through service methods. A topic model-as-a-service is online available for reviev 55 .

\footnotetext{
${ }^{54}$ https://github.com/librairy/modeler-service-facade/blob/master/src/main/avro/model. avpr

http://librairy.linkeddata.es/jrc-en-model
} 


\section{jrc-en-model ${ }^{\infty}$}

[ Base URL: librairy.linkeddata.es/jrc-en-model ]

http://librairy.linkeddata.es/jirc-en-model/v2/api-docs

Collection of legislative texts (EN) from the European Union generated between years 1958 and 2006

librAlry-Website

Send email to librAlry

Apache License Version 2.0

\section{/classes topic classification}

\section{/inferences topic distributions}

\section{/settings model details}

\section{/topics topic details}

\section{GET}

/topics list of topics

/topics/\{id $\}$ topic info

/topics/\{id $\} /$ neighbours topic neighbours

Figure 4.9: OpenAPI-based web interface of a probabilistic topic model service created with librAIry. 


\begin{tabular}{ll}
\hline \multicolumn{1}{c}{ Operations } & Tasks \\
\hline \hline $\begin{array}{l}\text { O1: reading of model hyperparameters (i.e } \\
\text { alpha, beta, and number of topics) }\end{array}$ & T1: Replication \\
\hline $\begin{array}{l}\text { O2: reading of pre-processing tasks (i.e stop- } \\
\text { words, normalization, PoS filtering) }\end{array}$ & \\
\hline $\begin{array}{l}\text { O3: reading of learning parameters (i.e iter- } \\
\text { ations, seed, likelihood) }\end{array}$ & \\
\hline $\begin{array}{l}\text { O4: reading of topics described by word dis- } \\
\text { tributions (i.e topic relevance) }\end{array}$ & T2: Exploration \\
\hline $\begin{array}{l}\text { O5: reading of words described by topic dis- } \\
\text { tributions (i.e word relevance) }\end{array}$ & T2: Exploration \\
\hline $\begin{array}{l}\text { O6: calculation of topic distributions in texts } \\
\text { (i.e vector of topic distributions) }\end{array}$ & T3: Inference \\
\hline
\end{tabular}

Table 4.2: Operations offered by a topic model-as-a-service to cover potential tasks.

\subsubsection{Reproducibility Tasks}

Through a single request to the model API, a list of parameters is provided to support the $\mathrm{O} 1, \mathrm{O} 2$, and $\mathrm{O} 3$ operations. The method is available by both a HTTP-GET request on /settings resource and by a TCP request on getSettings method. An online example is available here 56 .

A detailed list of the parameters provided by the topic model is shown below:

- algorithm: method used to train the model (e.g. LDA).

- date: when the model was created. It follows the ISO-8601.

- params: configuration of the learning process.

- seed: numerical value to ensure consistent results (e.g. 1066).

- lowercase: if true, text is converted to lowercase.

- topics: number of topics.

- language: language of texts.

- iterations: number of sampling iterations.

\footnotetext{
${ }^{56}$ http://librairy.linkeddata.es/jrc-en-model/settings
} 
- entities: if true, NER tasks are performed.

- max-doc-ratio: maximum word presence per document ratio (e.g. 0.9).

- min-freq: minimum word presence per number of document (e.g. 5).

- alpha: prior distribution over topic weights in each document (e.g. 0.1)

- beta: prior distribution over word weights in each topic (e.g. 0.01)

- part-of-speech: word classes used in the model (e.g. NOUN, VERB, ADJECTIVE)

- top-words: number of words used to describe a topic (e.g. 10)

- stop-words: list of words removed from the corpus (e.g. quantity, datum)

- stats: statistics after the learning process.

- loglikelihood: how much the model fits with the training set.

- vocabulary: number of unique words.

- topic-coherence: distance between topics from their top words (e.g min, max, mean, mode).

- topic-distance: distance between topics from their word distributions (e.g min, max, mean, mode).

- corpus: number of documents in the training set.

\subsubsection{Exploration Tasks}

The exploration task, with its respective operations of reading topics (O4) and words (O5), is broken down into four services:

- Topic List(O4). By means of the HTTP-GET / topics 57 resource and the TCP getTopics method, all the topics of the model are listed. Each topic is described with an increasing unique identifier (from 0 to the maximum number of topics), a label or name in case it has been established, and a description with the most relevant words (based on their density distributions).

\footnotetext{
${ }^{57}$ http://librairy.linkeddata.es/jrc-en-model/topics
} 
- Topic Detail(O4). By means of the HTTP-GET/topics/_id_[8 resource and the TCP getTopic method, a topic identified by $i d$ is described by providing its identifier, name, description and entropy (i.e how different is with respect the other topics).

- Topic Words(O5). By means of the HTTP-GET/topics/_id_/words $[9$ resource and the TCP getTopicWords method, a list of word distributions for the topic identified by $i d$ is provided. Every word has its weight (i.e relevance score) with respect to the topic.

- Topic Neighbours(O4). By means of the HTTP-GET/topics/_id_/neighbours 0 resource and the TCP getTopicNeighbours method, a list of topics related to the topic identified by $i d$ is provided. The distance between topics is measured from their word distributions.

\subsubsection{Inference Tasks}

A single request with the text to be analyzed returns a list with the presence of each topic (O5) in that text. The method is available by both a HTTP-POST request to Iinferences or a TCP request to the createInference method, with a JSON message containing the text to be analyzed.

\subsection{Summary}

In Section 4.1 we have described librAIry, the framework to cover the entire topic model life-cycle in a scalable way. Algorithms and tools coming from different technologies work collaboratively to process and analyze huge collections of textual resources creating and using probabilistic topic models.

We tested and validated librAIry by using the framework in some real world scenarios such as DrInventor 61 , where thousands of scientific publications were processed,

\footnotetext{
${ }^{58}$ http://librairy.linkeddata.es/jrc-en-model/topics $/ 0$

59 http://librairy.linkeddata.es/jrc-en-model/topics/0/words

60 http://librairy.linkeddata.es/jrc-en-model/topics/0/neighbours

${ }^{61}$ http://drinventor.eu
} 
TheyBuyForYou 62 , where hundreds of thousands of public procurement texts were analyzed, or CorpusViewer ${ }^{63}$, where millions of patents were automatically organized. Thus, librAIry has proven to be a valid and scalable text processing framework and addresses the first technical objective of this thesis ( T01, create a scalable platform for topic modeling).

librAIry has been designed to represent corpora by organizing the data in three levels of detail: snippets to reflect parts or pieces of texts, documents to represent full texts, and domains to group documents. Transversally there are annotations, which allow providing more details to any of them. Figure 4.2 shows how two scientific articles published in the same conference and that mention a same resource in their papers can be represented. On this representation model based on SEDA architectures, actions over resources and status change notification events are introduced, which enable to distribute the processing of resources. Figure 4.7 shows the four modules involved in processing the resources. Harvester modules create new documents, snippets and domains. Annotator modules react to each new resource and introduce annotations. Modeler modules create new topic models for each new domain. And Admin modules perform administrative tasks and allow users to read the data. As shown in figure 4.8, modules coordinate their actions by reacting to the notifications. The actions are then executed in parallel through a distributed workflow and the first research objective of this thesis is addressed (RO1, define a distributed text-processing model for creating large probabilistic topic models).

In Section 4.2 we propose the publication of topic models as web services that can be used from external solutions or integrated into the librAIry framework. Regardless of their API, since they work both over HTTP and over TCP, there are three types of tasks that guide the definition of the service: reproducibility, exploration, and inference. Tables 4.1 and 4.2 detail the operations supported by the service to cover these tasks. The definition of a topic model-as-a-service covers the second research objective of this thesis (R02, define a template to package probabilistic topic models as web services).

And finally, in order to facilitate the reuse of the topic models published as web services, section 4.2 .1 presents an online repository based on virtual services. This

\footnotetext{
62 https://theybuyforyou.eu

${ }^{63}$ https://www.plantl.gob.es/tecnologias-lenguaje/actividades/plataformas/Paginas/ corpus-viewer.aspx
} 
covers the second technical objective of the thesis (T02, create a repository of topicbased web services). 


\section{Chapter 5}

\section{Explainable Topic-based Associations}

As stated in Chapter 3, one of our hypotheses aims to determine whether it is possible to semantically relate texts taking into account their most relevant topics (H1.2). In particular, our goal is to determine whether two documents can be related by identifying their most representative topics.

However, as seen in Section 2.4.2, interpreting how documents are related from their topic distributions is hard when using density-based measures (e.g. distance equals to 0.74 or 0.76 ). The same pair of documents may vary their distance from each other when using topic models with different dimensions to represent them, as shown in figure 2.3. High dimensional models create more specific topics than models with fewer dimensions, and this topic specificity influences the way in which topic distributions are related, and consequently how documents can be related.

In order to better understand the relations derived from topic distributions, Section 5.1 compares scientific articles from their representations based on full-content, abstracts (i.e manual summaries), or summaries created automatically. Two types of metrics are considered: (i) internal-representativeness, focused on describing the content, and external-representativeness, focused on discovering relations (BadenesOlmedo et al., 2017a).

In Section 5.2, once we know how the topics are used to represent and relate texts, we propose a new topic-based annotation based only on the most representative topics, and a new distance measure that takes advantage of these representations (Badenes- 
Olmedo et al., 2017c). The main goal is not only to cluster texts through their most relevant topics, but also to facilitate the interpretation of their relations.

\subsection{Topic-based Relations}

This section studies the ability of topic distributions to capture the representativeness of a text through the relations that can be derived from it. In particular, it examines the performance offered by topic-based representations to describe scientific articles from their full-texts compared to representations based on summaries (e.g. abstract). The objective is twofold, to analyze comparisons based on topic distributions on the one hand, and to identify strengths and weaknesses when using abstracts to compare scientific articles on the other hand. Two novel measures are proposed based on the capability of the summary to substitute the original paper (Figure 5.1): (1) internalrepresentativeness, which evaluates how well the summary represents the original fulltext and (2) external-representativeness, which evaluates the summary according to how the summary is able to produce a set of related texts that are similar to what the original full-text has triggered.

Some studies (for Pharma and Sciences, 2016, Westergaard et al. 2017) have shown that text mining of full research articles give consistently better results than using only their corresponding abstracts. Given the size limitations and concise nature of abstracts, they often omit descriptions or results that are considered to be less relevant but still are important in certain Information Retrieval tasks. Thus, when other researchers cite a particular paper, $20 \%$ of the keywords that they mention are not present in the abstract (Divoli et al., 2012).

An analysis about the representativeness of research article summaries based on topic distributions is presented in this section, considering those based exclusively on abstracts and those based on their discursive structure (approach, challenge, background, outcomes and future work)(Simone T., 2010). The representativeness of a summary with respect to the original full-text is assumed as the degree of relation with the original one (internal-representativeness), along with the capacity of mimicking the full text when finding related items (external-representativeness). In order to quantify this notion of internal-external representativeness, a probabilistic topic model is trained to have a vectorial representation of each text retrieved from a paper: full content-based 


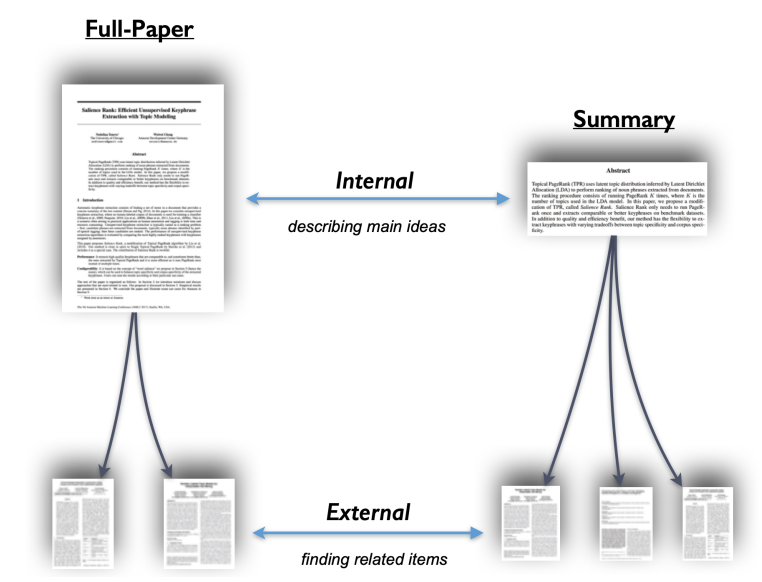

Figure 5.1: Internal and External Representativeness.

and summary-based (Figure 5.1). The vectorial representations of full-papers is used to measure the distance between them and those derived from abstract or summaries (internal-representativeness), and also to find similar documents (external- representativeness) based on the distance between their vectorial representations. An upper distance threshold is specified to filter less similar pairs and compose a set of related papers for each paper. Then, a comparison in terms of precision and recall is performed between sets obtained by only using the vectorial representation of full-papers, against sets produced by using other kind of summaries.

\subsubsection{Research Articles Summaries}

Some approaches have been proposed to summarize scientific articles (Cohan and Goharian, 2015) taking advantage of the citation context and the document discourse model. We have used the scientific discourse annotator proposed by (Ronzano and Saggion, 2015) to automatically create summaries from research papers by classifying each sentence as belonging to one of the following scientific discourse categories: approach, challenge, background, outcomes and future work. These categories were identified from the schemata proposed by (Teufel et al., 2009) with an original purpose of characterizing the content of Computer Graphics papers.

The annotator is based on a Support Vector Machine classifier that combines both lexical and syntactic features to model each sentence in a paper. The too 64 was in-

\footnotetext{
${ }^{64} \mathrm{http}: / /$ backingdata.org/dri/library/
} 
tegrated in our librAIry framework through the Rhetoric Module 65 to automatically annotate research papers with their rhetorical content.

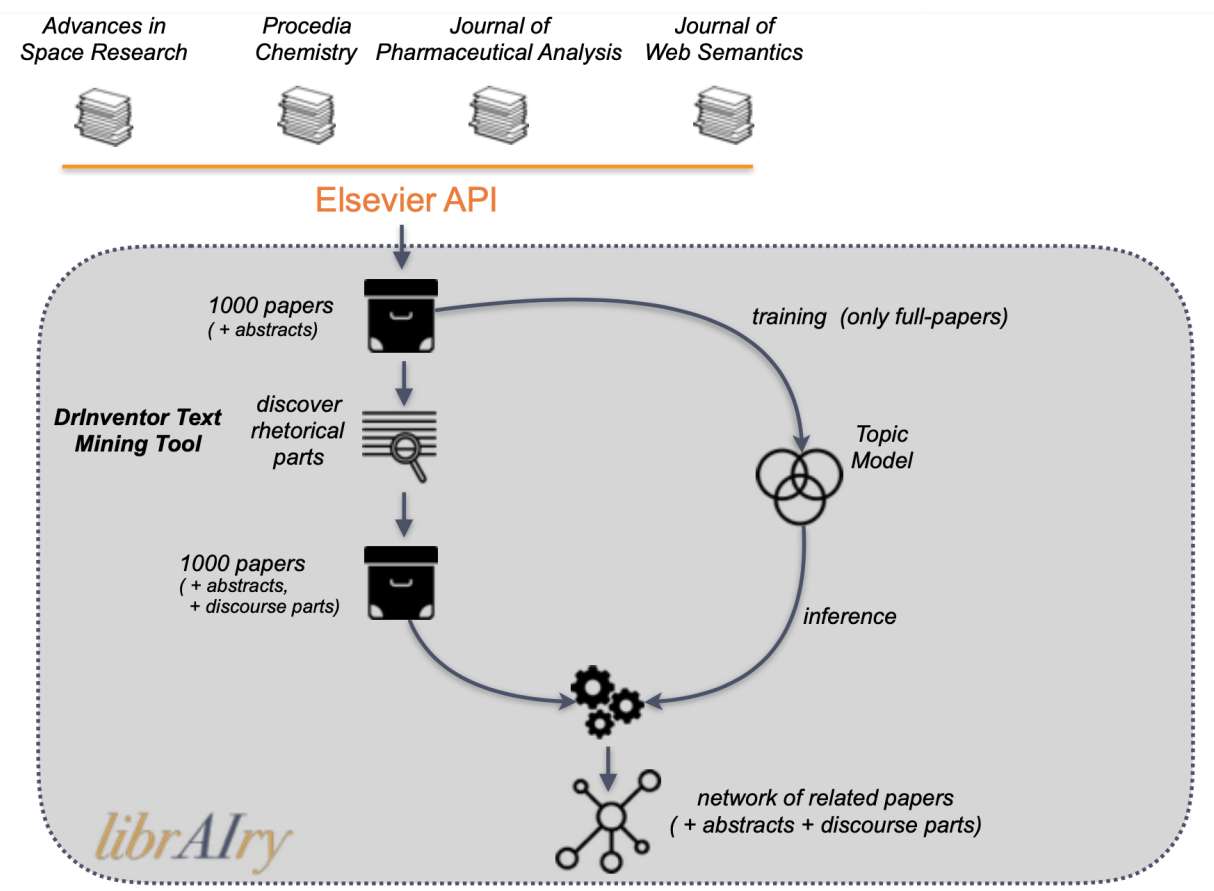

Figure 5.2: Experiment to analyze the ability of topic-based representations to create relations from summaries vs full-texts.

\subsubsection{Feature Vectors}

A representational model is required not only to measure distances between text fragments but, more importantly, to help to understand the differences in their content. As seen in Section 2.4.2, topic models are widely used to uncover the latent semantic structure from text corpora. In particular, Probabilistic Topic Models represent documents as a mixture of topics, where topics are probability distributions over words. Latent Dirichlet Allocation (LDA)(Blei et al., 2003) is the simplest generative topic model that makes it possible to characterize documents not previously used during the training task. This is a key feature for our evaluations because, although the model used for the experiments will be trained from the full-content of papers, it will be also used to describe the texts summaries.

\footnotetext{
${ }^{65}$ https://github.com/librairy/annotator-rhetoric
} 
Thus, we have used a LDA model to describe the inherent topic distribution of papers in the corpus. Some hyper-parameters need to be estimated: the number of topics $(k)$, the concentration parameter $(\alpha)$ for the prior placed on documents' distributions over topics and the concentration parameter $(\beta)$ for the prior placed on topics' distributions over terms. Since the target of this experiment is not to evaluate the quality of the representational model, but to compare their topic distributions, we accepted as valid values those widely used in the literature: $\alpha=0.1, \beta=0.1$, and $k=2 * \sqrt{n / 2}=44$ where $n$ is the size of the corpus.

\subsubsection{Similarity Measure}

Feature vectors in Topic Models are topic distributions expressed as vectors of probabilities. Hence we opt for Jensen-Shannon divergence (JS) (See Section 2.2) instead of the commonly used Kullback-Liebler divergence (KL). The reason for this is that KL is not defined when a topic distribution is zero and is not symmetric, what does not fit well with semantic similarity measures which in general are symmetric (Rus et al., 2013). JS considers the average of the distributions as follows :

$$
J S(p, q)=\sum_{i=1}^{T} p_{i} * \log \frac{2 * p_{i}}{p_{i}+q_{i}}+\sum_{i=1}^{T} q_{i} * \log \frac{2 * q_{i}}{q_{i}+p_{i}}
$$

where $T$ is the number of topics and $p, q$ are the topics distributions.

And the similarity measure used in our analysis is based on the JS transformed into a similarity measure as follows (Dagan et al. 1999) :

$$
\operatorname{similarity}\left(D_{i}, D_{j}\right)=10^{-J S(p, q)}
$$

where $D_{i}, D_{j}$ are the documents and $p, q$ the topics distributions of each of them.

\subsubsection{Evaluation}

The corpus used in the experiments was created by combining journals in different scientific domains such as Advances in Space Research, Procedia Chemistry, Journal of Pharmaceutical Analysis and Journal of Web Semantics (Figure 5.2). In total 1,000 papers were added, 250 from each journal. Both the abstract and the full-content of 
these documents were directly retrieved from the Elsevier API ${ }^{66}$ by using our Harvester module 67 The code used to perform the analysis along with the results obtained are publicly available 68

Since the annotation process to automatically discover the rhetorical parts of a research paper (Section 5.1.1) is sensitive to the structure of the phrases that are used when writing the text, only $20 \%$ of papers in the corpus could be fully annotated with all the fragments considered. In fact, these categories are not present in the same proportion in the corpus: approach (90\%), background (78\%), outcome (73\%), challenge (57\%) and future work (21\%)

\subsubsection{Internal Representativeness}

The internal-representativeness of a text measures the similarity of a summary against the original full-text. This measure is based on the JS between the topic distribution of each of them.

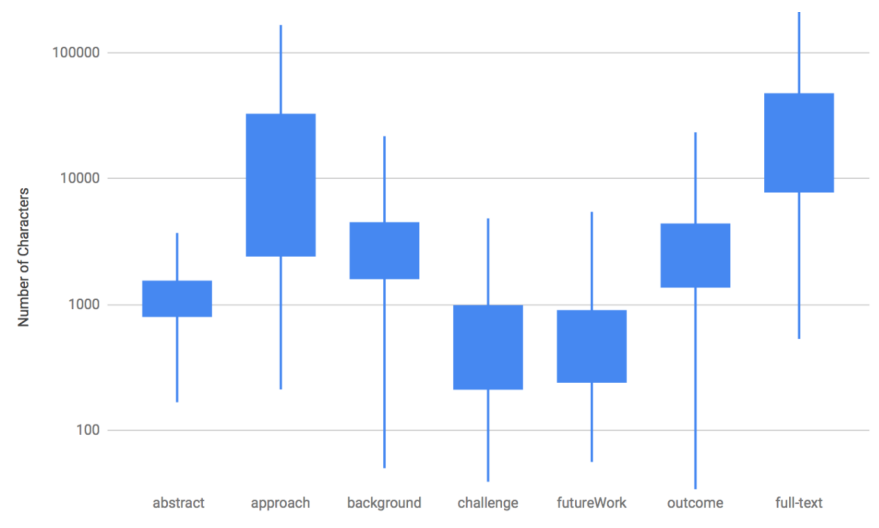

Figure 5.3: length of summaries.

Since LDA considers documents as bag-of-words, the text length (e.g. full-content or summaries) affects the accuracy of the topic distributions inferred by the topic model described in Section 5.1.2. The occurrences of words in short texts are less discriminative than in long texts where the model has more word counts to know how words are related (Hong and Davison, 2010). In view of the above, the approach, the background

\footnotetext{
${ }^{66}$ https://dev.elsevier.com

${ }^{67}$ https://github.com/librairy/harvester-elsevier

${ }^{68}$ https://github.com/librairy/study-semantic-similarity
} 


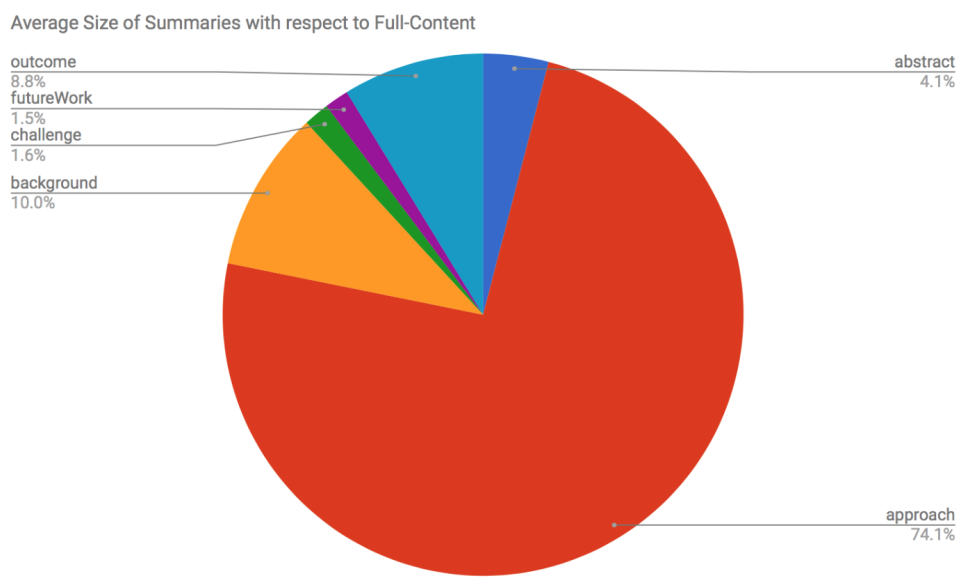

Figure 5.4: length of text parts.

and the outcome content of a paper generate more accurate topic distributions than those created from other approaches such as the abstract (Figure 5.3). Also, the relative presence of each of them in a paper (figure 5.4 shows an unexpected result when compared to the IMRaD format (Ramachandran and Vimala, 2014). This style proposes to distribute the content of an abstract, and by extension the full-paper, as follows: Introduction(25\%), Methods(25\%), Results(35\%) and Discussion(15\%). However, the results (figure 5.4 show that Method section (approach content) is more extensive than Results section (outcome content) in our corpus.

All pairwise similarities between full-papers, abstracts and rhetorical-based summaries are calculated to measure the internal-representativeness of a summary with respect to the original text, i.e. the topic-based similarity value (equation 5.2 between the probability distributions of the full-text and each of the summaries. Results (table 5.1) suggest that summaries created from the approach content are more representative than others, i.e. the distribution of topics describing the text created from the approach content is the most similar to the one corresponding to the full-content of the paper.

\subsubsection{External-Representativeness}

The external-representativeness metric tries to measure how different is the set of related documents obtained from summaries with respect to those derived from the 


\begin{tabular}{lcccccc}
\hline & Min & Lower Quartile & Upper Quartile & Max & Dev & Median \\
\hline abstract & 0.0489 & 0.9109 & 0.9840 & 1.0000 & 0.1443 & 0.9741 \\
approach & $\mathbf{0 . 0 4 9 9}$ & $\mathbf{0 . 9 9 6 9}$ & $\mathbf{1 . 0 0 0 0}$ & $\mathbf{1 . 0 0 0 0}$ & $\mathbf{0 . 0 8 7 2}$ & $\mathbf{0 . 9 9 9 8}$ \\
background & 0.0463 & 0.8967 & 0.9937 & 0.9988 & 0.2037 & 0.9822 \\
challenge & 0.0426 & 0.7503 & 0.9517 & 0.9940 & 0.2224 & 0.8829 \\
futureWork & 0.0000 & 0.6003 & 0.9435 & 0.9948 & 0.2842 & 0.8814 \\
outcome & 0.0485 & 0.9267 & 0.9925 & 0.9990 & 0.1721 & 0.9835
\end{tabular}

Table 5.1: Accuracy results when comparing the most related articles using a summary (e.g. abstract, approach, background, challenge, future work or outcome), with those obtained using the full-text of the article (internal-representativeness).

original full-text. In terms of precision, recall and f-measure, a comparison has been performed to analyze the behavior of the summaries when trying to discover related content compared to use the full-text of the article.

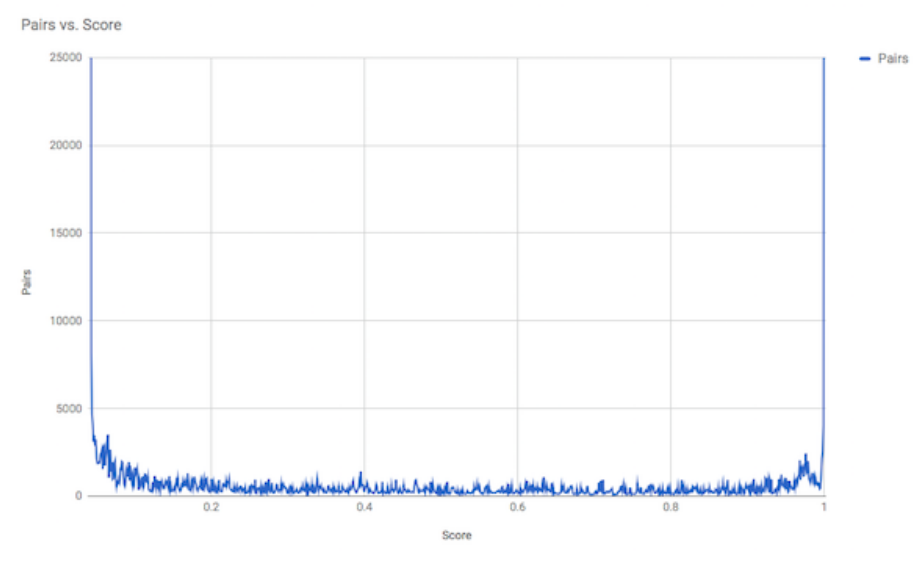

Figure 5.5: number of pairwises by similarity score (rounded up to two decimals).

By using the same topic model previously created, similarities among all pairs of documents were also calculated according to equation 5.2. Then, a minimum score or similarity threshold is required to define when a pair of papers are related. Each threshold is used to create a gold-standard which relates articles to others based on their similarity values. In order to discover that lower bound of similarity, a study about trends in the similarity scores (fig 5.5) as well as distributions of topics in the corpus (fig 5.6) was performed. We can see that topics are not equally balanced across papers. This fact generates separated groups of strongly related papers. We think 
this phenomena is due to our usage of a corpus created from journals where different domains are equally balanced. Then, we considered a similarity score equals to 0.99 (fig 5.5) as the threshold from which strong relations appear. However, to cover different interpretations of similarity, from those based on sharing general ideas or themes to those that imply to share a more specific content, the following list of thresholds was considered in the experiments: $0.5,0.6,0.7,0.8,0.9,0.95$ and 0.99 .

For each similarity threshold, a gold-standard was created based on considering as related those papers with a similarity value upper than the selected threshold. Results ( figure 5.7) comparing the related papers inferred from the full-content with those inferred from the partial-content representation (i.e. abstract or rhetorical parts) suggest that strongly related papers are mainly discovered by using the summary created from the approach section. The reason for this may be based on the average size of this type of summaries or the particular content included in this part of a paper. While other summaries include more general-domain words, the approach content includes more specific words that describe the method or the final objective of the paper. So, for higher similarity thresholds, i.e. for strongly related papers, the recommendations discovered by using the approach are more precise than those discovered by using the abstract.

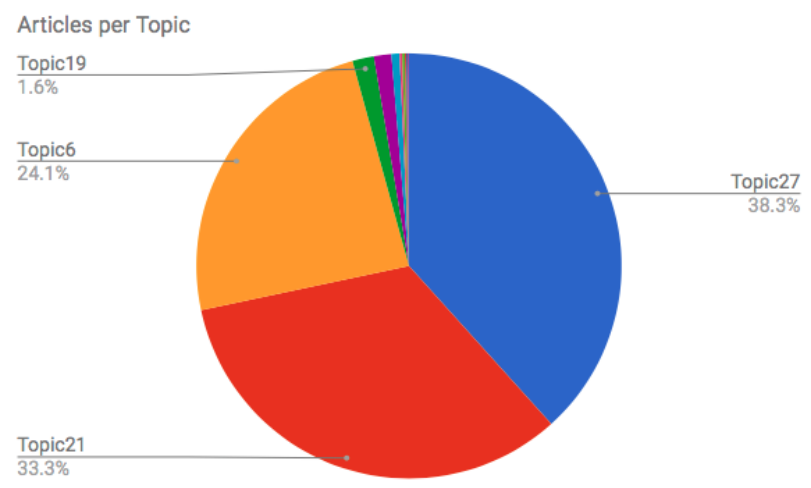

Figure 5.6: topics per article with value above 0.5 .

In terms of recall (figure 5.8), the upward trend followed by the approach, the outcome and the background content remarks the assumption of summaries containing key words allow to discover more similar papers than others. Moreover, since recall overlooks false-negatives classifications, it suggests that these parts of a research paper 
share more words than others with strongly related papers but they may also present commonalities with highly related papers, except in case of approach which still exhibits higher precision.

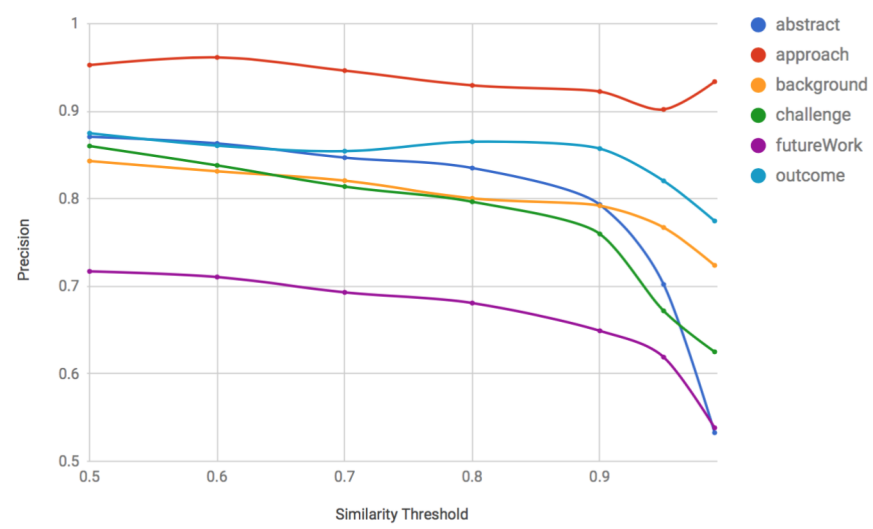

Figure 5.7: Precision at different similarity thresholds.

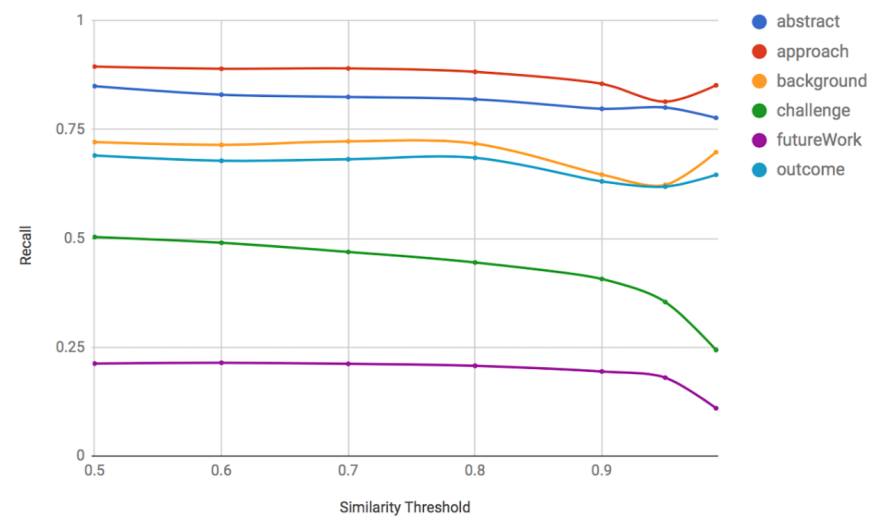

Figure 5.8: Recall at different similarity thresholds.

As expected, only summaries created from the approach, the outcome and the background content maintain high accuracy values (fig 5.9 ) even for high similarity thresholds. Along with the results showed in figure 5.10, where the same three rhetorical classes present the lowest standard deviation over the f-measure, they can be considered as the most robust summaries containing the ideas that better characterize the paper compared to others. 


\subsubsection{Conclusion}

Topic-based relations have been studied among scientific documents based on their abstract sections with respect to summaries corresponding to their scientific discourse categories. For this purpose, two novel measures have been proposed: (1) internalrepresentativeness and (2) external-representativeness.

Results show that summaries created from the approach, outcome or background content of a paper describe more accurately its full-content in terms of overall ideas and related documents than abstracts. Although those summaries are more extensive in number of characters than other with similar precision such as the abstract content, they have proven to be particularly helpful discovering strongly related papers, i.e. papers with a similarity value close to 1.0 .

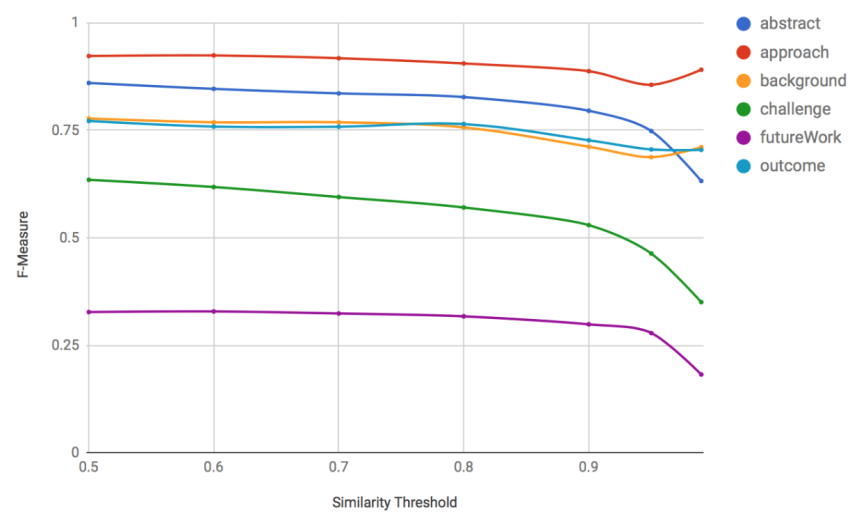

Figure 5.9: f-measure performance.

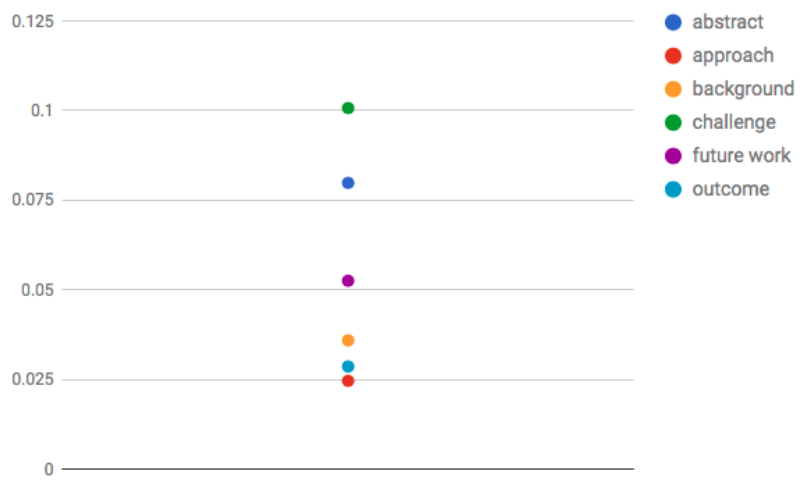

Figure 5.10: f-measure deviation. 


\section{$5.2 \quad$ Topic-based Clustering}

Once we have a better understanding of the behavior of topic models to represent texts, and the relationships that can be derived from them, we examine their usefulness in browsing document collections. A way to explore the knowledge inside document collections is by moving from one information element to another based on certain criteria that relates them. This approach requires to calculate a similarity matrix with all possible comparisons between elements, so we can later select the most pertinent ones. Since computing a $n \times n$ matrix takes $O\left(n^{2}\right)$ time, obtaining all possible pairs of similarities in a large collection of documents can be unfeasible because of the quadratic cost of comparing every pair of elements.

In order to reduce the complexity, some approaches have introduced mechanisms (mainly pre-election methods) to alleviate the problem of making this calculation over the whole set of pairs in the collection. However those methods are still quite costly.

In this section we propose a novel clustering technique based on topic model distributions that reduces the complexity to find relations between documents in a large corpus of textual documents, without compromising efficiency and providing additional information about relations. A detailed description of our algorithm is given in Section 5.2.1. The experiments to verify the efficiency and effectiveness of our clustering algorithms using real data, and demonstrate that our approach is competitive enough against both a centroid-based and a density-based clustering baselines are described in Section 5.2.2. The most relevant results and conclusions are finally presented in Section 5.2 .3 .

\subsubsection{Most Relevant Topics}

Our algorithm to identify the most relevant topics draw inspiration from other clustering techniques to divide the initial space of elements into smaller sub-groups where the complexity of calculating all possible distances is significantly reduced. Existing unsupervised approaches based on centroids or density measures require to make comparisons between elements to find groups of similar elements in the collection. They usually follow an iterative methodology to produce the final solution, based on calculating distances between the elements inside each intermediate state. A naïve approach would need to calculate all possible distances between elements, which takes $O\left(n^{2}\right)$ time 

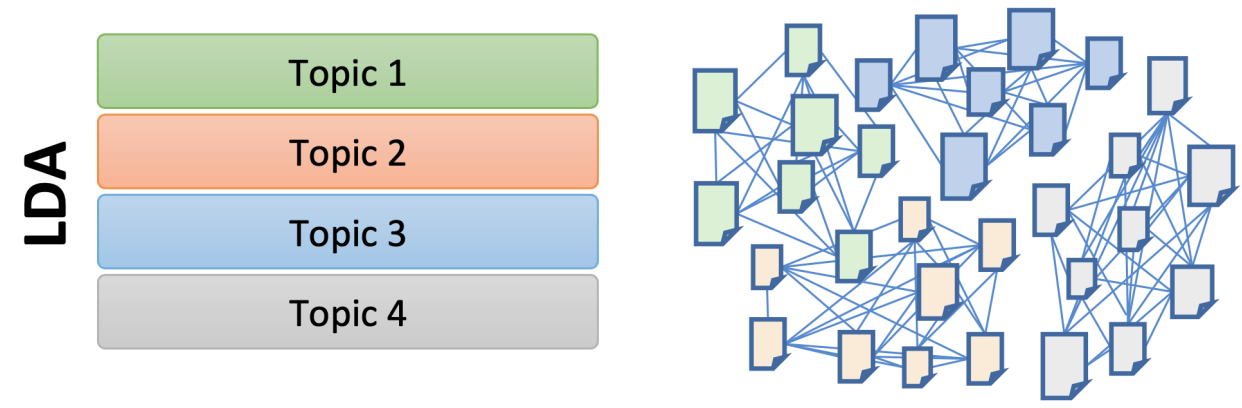

Figure 5.11: Probabilistic Topic Models, and in particular Latent Dirichlet Allocation (LDA), can efficiently divide the search space and speed up the process of finding relations among documents inside big collections.

for a $n \times n$ matrix. That makes it impossible to apply such techniques on large collections of documents, since the cost of comparing each element with the others escalates quickly. For those big volumes of data, a clustering task that only takes linear time to discover the clusters can significantly alleviate this problem. For example, a classification method that does not require any other data except the element information to assign the item to the corresponding cluster will take $O(n)$ time to compose those groups.

The classification method needs to take advantage of both the vectorial representations of the documents and the similarity measure used to relate them in a corpus. Since the representational model considered is based on Probabilistic Topic Models (and more specifically on LDA), the classification method leverages on the particular behavior of Dirichlet distributions, which describes each document by a density vector where the sum of all the probability values must be equal to 1.0. Thus, analyzing the relations between the topics that compose a topic distribution becomes more important than comparing their probability values with another topic distribution.

Our hypothesis is that, given a collection of topic distributions, an unsupervised classification with high precision and linear computing time can be performed by considering only the topic distribution of each document and without needing to further compare it with other document's distributions.

All algorithms have been compared in terms of cost, effectiveness and efficiency (Halkidi et al., 2001). Cost is based on the number of pairwise similarity checks. 
Effectiveness handles relevance measures such as precision and recall. And efficiency tries to measure the overall balance between cost and effectiveness. More details about those measures will be included in Section 5.2 .2 ,

\subsubsection{Trends-based Clustering Method}

Topic distributions are formalized as probability distributions following a Dirichlet distribution, so their probability values sum to 1 . In this way, the relevance of a topic is influenced and at the same time influences the relevance of the others items in the distribution. Our first approach named Trends on Dirichlet distribution-based Clustering (TDC) considers changes in the relevance, i.e. probability values of the topics instead of directly relying on the scores associated to a given topic distribution (Figure 5.12). It expresses the oscillations between topic weights considering a fixed order between them. The order can be any, as long as it remains constant in all distributions. Thus, a probability-vector composed by $n$ density values is translated to a trend-expression made out of $n-1$ trend-values such as (1) upward, (2) downward and (0) sustained. This trend-expression will identify the cluster the distribution falls into, and therefore the corresponding item belongs to. TDC is defined as:

$$
T D C(P)=T
$$

where: $T_{i}=1$, when $P_{i}<P_{i+1}$

$T_{i}=2$, when $P_{i}>P_{i+1}$

$T_{i}=0$, when $P_{i}=P_{i+1}$

For example, given the distribution $P_{1}=[0.23,0.18,0.33,0.13,0.13]$, the assigned cluster will be $T=2120$. The first value is 2 because 0.23 is greater than 0.18 (same for other values).

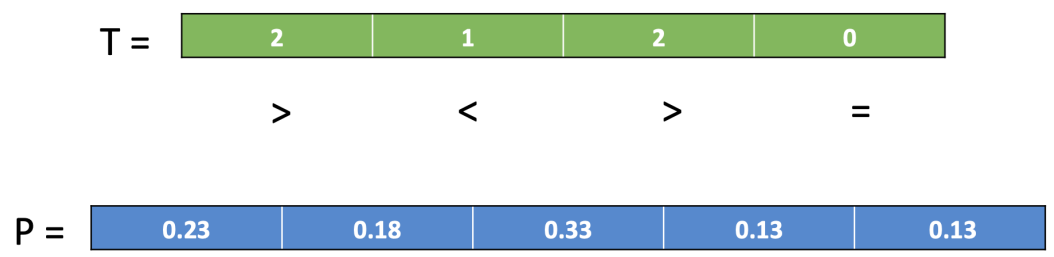

Figure 5.12: TDC considers variations across consecutive topics inside a document's topic distribution. 


\subsubsection{Ranking-based Clustering Method}

We propose a clustering technique named Ranking on Dirichlet distribution-based Clustering (RDC) that only considers the top $n$ topics from the ranked list of probability distributions to classify similar topic distributions (Figure 5.13). It is based on the focal document selection proposed by (Towne et al., 2016) to validate LDA-based similarity algorithms against human perception of similarity. RDC is defined as:

$$
R D C(P)=R
$$

where $\forall i \in R, R_{i}>=R_{i+1}$ and $\forall j \in P, R_{1}>=P_{j}$

This is based on the assumption that the highest weighted topics have a high influence in the rest of topics in terms of calculating distances, when comparing continuous multivariate probability distributions. Since similarity measures (Section 2.2 based on probability distributions are oriented to determine the uncertainty of the distribution, when a mixture of probability distributions is considered, as in the case of Topic Models, the top $n$ distributions (i.e. the most relevant topics) should be sufficient to allow us grouping similar distributions. Taking into account the above considerations, the RDC algorithm classifies a topic distribution according to only $n$ highest probability values. For instance, given the following topic distribution: $P_{2}=[0.23,0.18,0.33,0.13,0.13]$, the assigned cluster is 3 from RDC-1 because that is the topic with the highest weight.

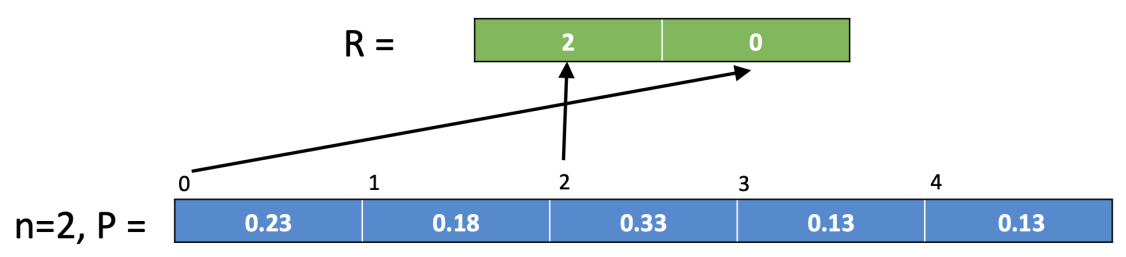

Figure 5.13: RDC only considers the top $\mathrm{n}$ topics from the ranked list of probability distributions.

\subsubsection{Cumulative Ranking-based Clustering Method}

A variant of the previous algorithm, named Cumulative Ranking on Dirichlet distributionbased Clustering (CRDC), also aims to discover the most representative topics that can help to group similar topic distributions. While RDC is based on a fixed number of 
topics, CRDC is based on the cumulative sum of the weights of the highest topics (Figure 5.14). The number of topics is now dynamically determined by a threshold, and once this threshold is reached no more topics are considered. CRDC is defined as:

$$
C R D C(P)=C
$$

where $\forall i \in C, C_{i}>=C_{i+1}$ and $\sum_{i=1}^{T} C_{i}>=w$ with $T$ size of $C$, and $w$ a cumulative weight threshold.

For instance, considering a CRDC algorithm with a cumulative weight threshold of 0.9 , and the following topic distribution: $P_{3}=[0.36,0.58,0.05,0.01]$. The assigned cluster will be 21. To come up with this cluster, a ranked list of topics based on their weights is first calculated, $R_{p}=2|1| 3 \mid 4$. Then, a sum of weights according to the order described by $R_{p}$ is performed. When the accumulated sum is greater than the threshold, the topics taking part of the sum will be selected to "label" the cluster. In this case, the cumulative weight threshold is 0.9 therefore using only the first two topics we exceed the threshold: $w=0.58+0.36=0.94$

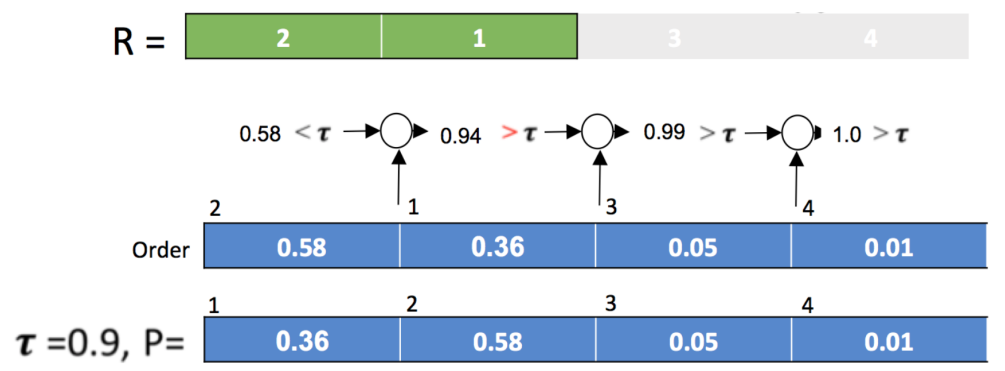

Figure 5.14: CRDC only considers the top $\mathrm{n}$ topics until the sum of the weights of the highest topics exceeded a given threshold.

\subsubsection{Evaluation}

In this section we present the experimental setup for evaluating our trends-based (TDC), ranking-based (RDC) and cumulative ranking-based (CRDC) clustering approaches, considering both JS divergence and He distance as similarity measures. We describe the datasets and baseline algorithms that will be used for comparison. 


\subsubsection{Datasets}

We used two datasets to evaluate the performance of the algorithms. The first dataset, DIRICHLET-RANDOM-MIXTURE (DRM), is synthetic69. To generate the dataset, we sampled $k$ probabilistic distributions from a randomly $k$-dimensional selector based on Dirichlet distributions. This implies that all probabilities must to sum to 1 for each sampled point. The number of sampled points from this mixture of Dirichlet distributions is $n=1000$.

The second dataset has been created from a collection of research papers published in the Advances in Engineering Software (AIES) journal. They were retrieved from the Springer API by using the librAIry (Badenes-Olmedo et al., 2017b) framework and a Topic Model based on LDA was created from them. The sample is also composed by $n=1000$ documents.

Topic models were trained from these datasets by using the criteria described by (Steyvers and Griffiths, 2006): $\alpha=50 / k, \beta=0.01$ and $k=2 *(\sqrt{(n / 2)})$, where $k$ is the number of topics and $n$ is the number of documents. Since both datasets contain 1000 documents $(n)$, the hyper-parameters $\alpha$ and $\beta$ are assigned as follow: $\alpha=1.136$, and $\beta=0.01$, and the number of topics is fixed to $k=44$. Further tuning of the settings is not crucial in this evaluation process, because we are not focusing on the quality of the model but on the efficiency when calculating similarities from their representational distributions.

\subsubsection{Settings}

Since there is no unified criteria to select a threshold inside the distance scores spectrum that allows us to determine when two documents are similar, we decided to study the distribution of similarity values calculated from all pairwise comparisons. In Figure 5.15, the result of grouping all similarities by the two most representative decimals, i.e. the first two decimals of the similarity value, is shown. Then, a polynomial function (red line) is approximated to describe the trend of these values. In this function, the similarity score 0.83 emerges as a global minimum and has been used for filtering out the non-similar document pairs.

\footnotetext{
${ }^{69}$ https://doi.org/10.5281/zenodo.931305
} 


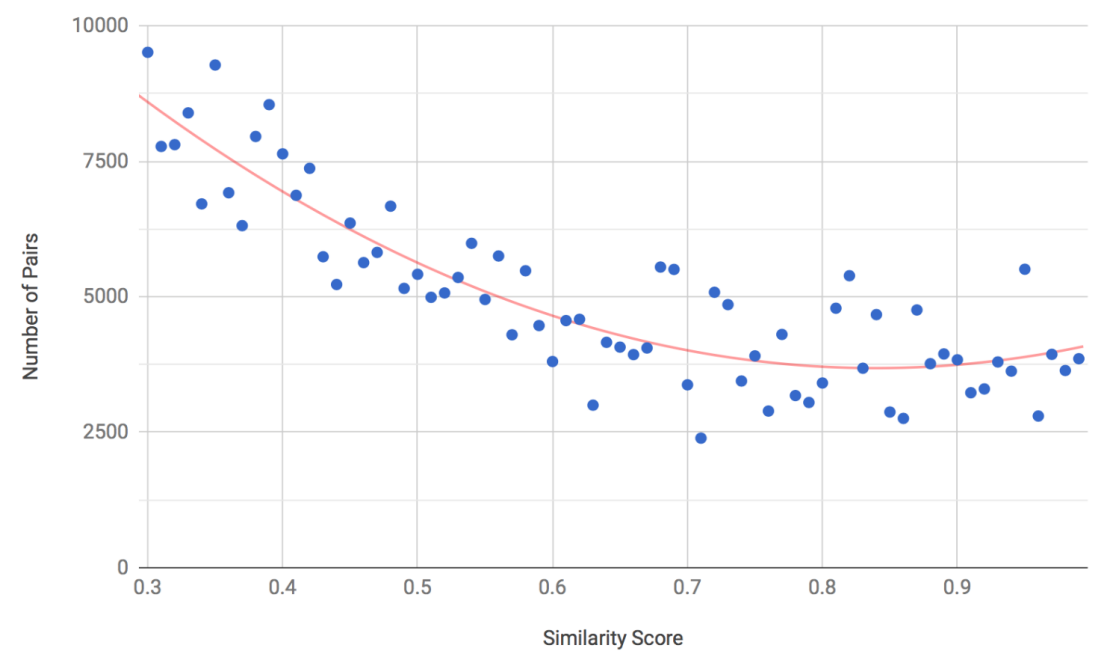

Figure 5.15: Similarity values grouped by frequency in AIES

\subsubsection{Baselines}

We compare the performance of TDC, RDC and CRDC algorithms against the following baselines:

- K-Means as a centroid-based clustering approach.

- $D B S C A N$ as a density-based clustering approach.

- Random, which randomly selects $R$ from the dataset

Initially, K-Means (Bahmani et al. 2012) randomly composes a set of centroids and assigns each point of the sample to its nearest cluster based on a distance measure. Then, a new set of centroids is calculated from the previous ones according to the assigned points. This process is repeated until the set of centroids does not change significantly between consecutive iterations or a maximum number of iterations is reached. The scalable K-Means approach used in our experiments is an improved version of $k$-means which obtains an initial set of centers ideally close to the optimum solution. The algorithm implemented at the Apache Commons Math library 70 was used in the experiments. Based on empirical results, the best configuration is: $k=$ number - of - topics $=44$ and maxIterations $=50$

\footnotetext{
${ }^{70}$ http://commons.apache.org/proper/commons-math/
} 
A widely known density-based algorithm is DBSCAN (Ester et al., 1996), which compose clusters from the neighborhood of each point considering at least a minimum number of points and a given radius. Thus, it requires to specify the radius of the point's neighborhood, Eps, and the minimum number of points in the neighborhood MinPts. Based on empirical results, the best results were obtained with the following configuration: $e p s=0.1$ and $\operatorname{minPts}=50$

The Random algorithm takes as input a parameter $m$ and randomly divides the dataset into $m$ equal-sized groups of similar documents. For the evaluation, $m$ was set to the number of topics, the dimension of the dataset.

With respect to the proposed algorithms and taking into account empirical results, the $\mathrm{RDC}$ algorithm is set to use the top 1 highest topics, and the cumulative weight threshold for the CRDC algorithm is set to 0.9 .

\subsubsection{Measures}

A gold-standard is created for each dataset and distance metric considered. They are created by calculating all pairwise similarities from their documents. Since the $n \times n$ similarity matrix requires $O\left(n^{2}\right)$ time to be calculated, the selected size of datasets has not been too large $n=1000$.

We considered three measures to evaluate our algorithms with respect to the baseline:

- cost: based on the number of similarity score calculations required by the algorithm:

$$
\text { cost }=(\text { reqSim }-\operatorname{minSim}) /(\text { totalSim }-\operatorname{minSim})
$$

The minSim corresponds to the number of similar documents obtained from using the threshold score previously mentioned in section 5.2.2.2. The totalSim corresponds to the Cartesian product of existing documents: totalSim $=n * n=$ 1,000,000. And the reqSim corresponds to the number of similarities calculated by the algorithm.

- effectiveness: based on precision and recall. It expresses the quality of the algorithm:

$$
\text { effectiveness }=\frac{\text { precision }^{2}+\text { recall }^{2}}{2}
$$


- efficiency: based on the previous ones, it express a compromise between quality and performance:

$$
\text { efficiency }=\text { effectiveness }- \text { cost }
$$

\subsubsection{Results}

The source code used to evaluate the algorithms along with the results obtained are publicy availablet1.

In terms of effectiveness (Figures 5.16 and 5.17), the results highlight that $K$ Means and $C R D C$ outperform the other algorithms. K-Means was expected to be a top performer because the algorithm itself performs comparisons to map clusters. The fact that $C R D C$ has such good performance encourages us to think that, in fact, the most relevant topics when they altogether exceed a certain high weight threshold, are those that best represent the document and allow to group together similar documents. However, as shown in tables 5.2, 5.3, 5.5 and 5.4, considering a fixed number of more relevant topics $(R D C)$ or considering the trend of their weights $(T D C)$ does not seem to perform so well on aggregating similar documents, since their precision and recall values are very low in both cases. It is surprising that the DBSCAN has such low value. Taking a look at its precision and recall values, and also seeing the number of groups that each algorithm has created (Figure 5.18), we believe that having a corpus containing a very cohesive set of documents (all papers in corpus belong to the same journal) affects the performance of this algorithm since it divides the corpus into a lower number of groups. This way, it obtains high values of recall because most of the pair-wise distances are computed, but very low precision.

The results also show that the behavior of the algorithms does not differ significantly when using different similarity measures, for example JS divergence (Figure 5.16) and He distance (Figure 5.17). This highlights the importance of the documents' topic distributions to successfully classify them into smaller groups of similar items, while other particular aspects such as the distance or similarity metric used to compare them are less influential.

In terms of cost (Figures 5.19 and 5.20), the best clustering algorithm, as expected, is based on random selection. This is due to the fact that the number of pairs compared

\footnotetext{
${ }^{71}$ https://doi.org/10.5281/zenodo.931305
} 


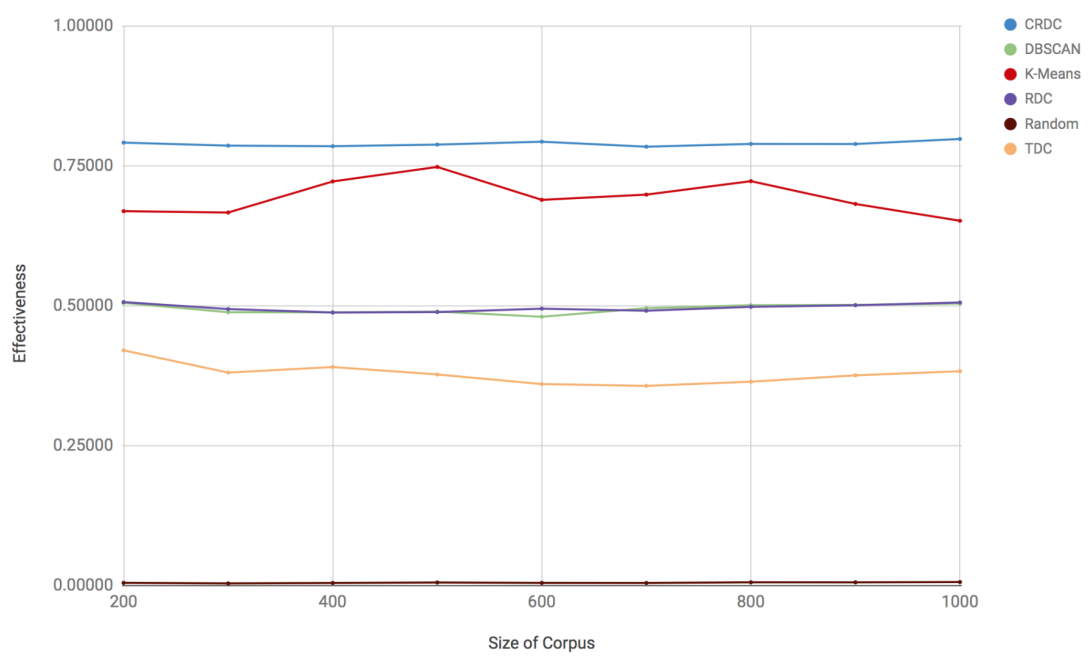

Figure 5.16: Effectiveness (JS-based) in AIES

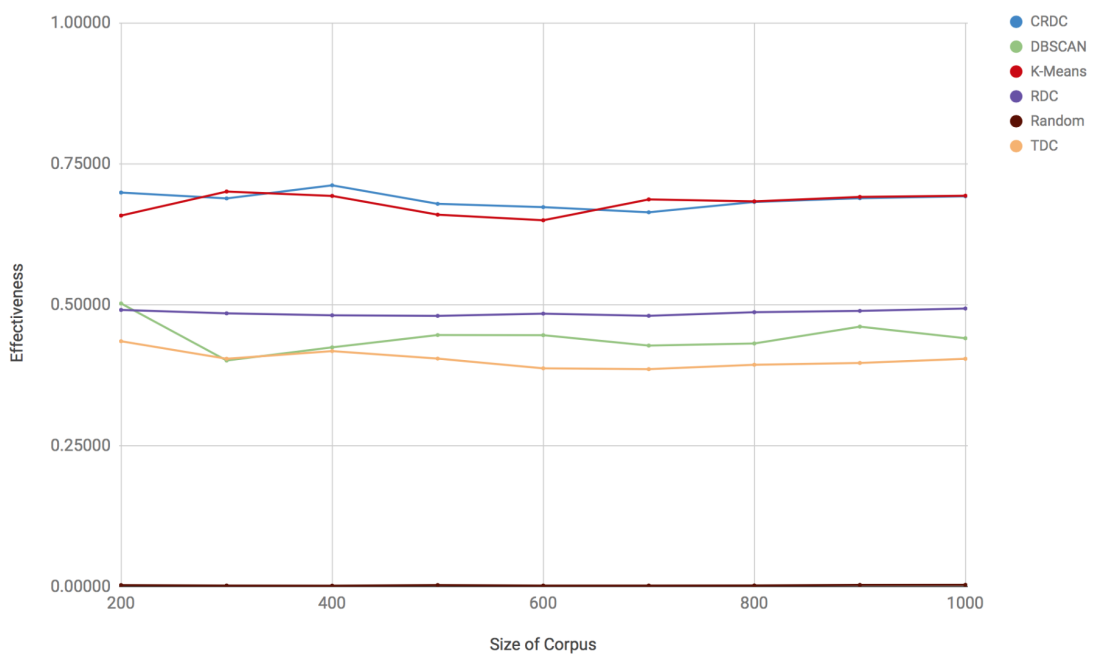

Figure 5.17: Effectiveness (He-based) in AIES

by this algorithm is always the minimum, given the dataset is simply randomly divided into $m$ equal-sized groups, where $m$ is equals to the number of topics, i.e. dimension of the dataset. Since K-Means and DBSCAN make comparisons between documents until their internal condition is satisfied, they are the most inefficient approaches. K-Means involves the highest cost because it compares all the documents with the 44 centroids in each iteration.

Among our proposals, the main reason for an algorithm to present a higher cost is 


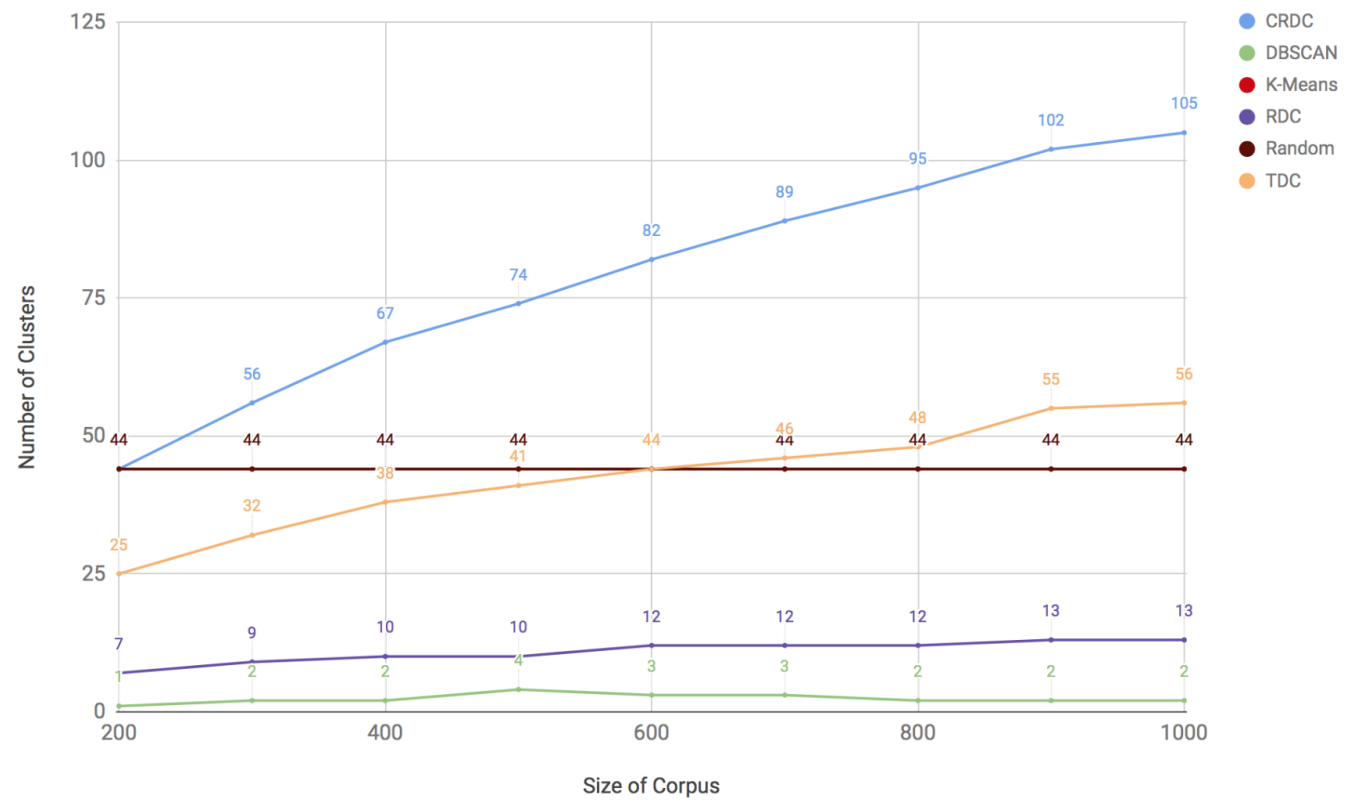

Figure 5.18: Clusters in AIES

\begin{tabular}{lcccccc}
\hline Size & CRDC & DBSCAN & K-Means & RDC & TDC & Random \\
\hline 200 & 0.94 & 0.10 & $\mathbf{0 . 9 6}$ & 0.31 & 0.42 & 0.12 \\
300 & 0.93 & 0.15 & $\mathbf{0 . 9 4}$ & 0.30 & 0.39 & 0.08 \\
400 & $\mathbf{0 . 9 3}$ & 0.15 & 0.89 & 0.29 & 0.39 & 0.09 \\
500 & $\mathbf{0 . 9 2}$ & 0.30 & 0.90 & 0.28 & 0.38 & 0.09 \\
600 & $\mathbf{0 . 9 2}$ & 0.19 & 0.88 & 0.28 & 0.38 & 0.08 \\
700 & $\mathbf{0 . 9 2}$ & 0.20 & 0.91 & 0.28 & 0.38 & 0.09 \\
800 & $\mathbf{0 . 9 2}$ & 0.12 & 0.89 & 0.30 & 0.39 & 0.10 \\
900 & $\mathbf{0 . 9 2}$ & 0.13 & 0.87 & 0.30 & 0.40 & 0.10 \\
1000 & $\mathbf{0 . 9 3}$ & 0.13 & 0.90 & 0.30 & 0.40 & 0.10
\end{tabular}

Table 5.2: Precision (JS-based) in AIES

due to the number of groups the corpus is divided into (see Figure 5.18). The greater the number of groups, the fewer the number of later comparisons that have to be made and, therefore, the lower the cost of the algorithm.

The behavior of the $D B S C A N$ algorithm depends remarkably on the similarity metric used. We think that this may be due to the way in which both measures satisfy the triangle inequality condition, since one is based on divergence (JS) and the 


\begin{tabular}{lcccccc}
\hline Size & CRDC & DBSCAN & K-Means & RDC & TDC & Random \\
\hline 200 & 0.75 & 0.07 & $\mathbf{0 . 8 4}$ & 0.23 & 0.08 & 0.33 \\
300 & 0.74 & 0.08 & $\mathbf{0 . 8 3}$ & 0.23 & 0.06 & 0.32 \\
400 & $\mathbf{0 . 7 6}$ & $\mathbf{0 . 0 9}$ & 0.76 & 0.22 & 0.06 & 0.32 \\
500 & 0.73 & 0.08 & $\mathbf{0 . 7 4}$ & 0.21 & 0.08 & 0.31 \\
600 & 0.72 & 0.08 & $\mathbf{0 . 7 3}$ & 0.21 & 0.06 & 0.30 \\
700 & 0.71 & 0.10 & $\mathbf{0 . 7 6}$ & 0.21 & 0.06 & 0.30 \\
800 & 0.73 & 0.11 & $\mathbf{0 . 7 8}$ & 0.22 & 0.07 & 0.31 \\
900 & 0.73 & 0.12 & $\mathbf{0 . 8 0}$ & 0.22 & 0.08 & 0.32 \\
1000 & 0.74 & 0.15 & $\mathbf{0 . 7 7}$ & 0.23 & 0.08 & 0.32
\end{tabular}

Table 5.3: Precision (He-based) in AIES

\begin{tabular}{lcccccc}
\hline Size & CRDC & DBSCAN & K-Means & RDC & TDC & Random \\
\hline 200 & 0.92 & $\mathbf{1 . 0 0}$ & 0.79 & 0.96 & 0.02 & 0.87 \\
300 & 0.91 & 0.89 & 0.84 & $\mathbf{0 . 9 6}$ & 0.02 & 0.84 \\
400 & 0.92 & 0.92 & 0.90 & $\mathbf{0 . 9 6}$ & 0.02 & 0.86 \\
500 & 0.91 & 0.94 & 0.88 & $\mathbf{0 . 9 6}$ & 0.03 & 0.85 \\
600 & 0.91 & 0.94 & 0.87 & $\mathbf{0 . 9 6}$ & 0.02 & 0.83 \\
700 & 0.91 & 0.92 & 0.90 & $\mathbf{0 . 9 6}$ & 0.02 & 0.83 \\
800 & 0.92 & 0.92 & 0.88 & $\mathbf{0 . 9 6}$ & 0.02 & 0.83 \\
900 & 0.92 & 0.95 & 0.86 & $\mathbf{0 . 9 6}$ & 0.02 & 0.83 \\
1000 & 0.92 & 0.93 & 0.89 & $\mathbf{0 . 9 7}$ & 0.02 & 0.84
\end{tabular}

Table 5.4: Recall (JS-based) in AIES

other on distance $(\mathrm{He})$. This property, which defines distance $(a, b) \leq \operatorname{distance}(a, c)+$ distance $(c, b)$, is very important in the calculations that DBSCAN makes to discover the groups, since it only calculates the distances between near points.

Finally, in terms of efficiency (Figures 5.21, 5.22), regardless of the similarity measure used, the algorithm that yields the best performance according to the results obtained is $C R D C$. Overall, $C R D C$ demonstrates a high accuracy classification and a lower cost by improving the performance offered by centroid-based or density-based approaches.

We have also created a synthetic dataset, DRM (Section 5.2.2.1), composed of 1000 Dirichlet distributions with the same dimensions than topics in AIES: $k=44$. 


\begin{tabular}{lcccccc}
\hline Size & CRDC & DBSCAN & K-Means & RDC & TDC & Random \\
\hline 200 & 0.84 & $\mathbf{1 . 0 0}$ & 0.65 & 0.96 & 0.02 & 0.82 \\
300 & 0.84 & $\mathbf{0 . 9 8}$ & 0.76 & 0.95 & 0.02 & 0.78 \\
400 & 0.84 & $\mathbf{0 . 9 8}$ & 0.79 & 0.94 & 0.02 & 0.79 \\
500 & 0.85 & 0.94 & 0.87 & $\mathbf{0 . 9 5}$ & 0.02 & 0.78 \\
600 & 0.86 & $\mathbf{0 . 9 6}$ & 0.80 & 0.95 & 0.02 & 0.76 \\
700 & 0.85 & $\mathbf{0 . 9 8}$ & 0.80 & 0.95 & 0.02 & 0.76 \\
800 & 0.85 & $\mathbf{0 . 9 9}$ & 0.81 & 0.95 & 0.02 & 0.76 \\
900 & 0.85 & $\mathbf{0 . 9 9}$ & 0.75 & 0.95 & 0.02 & 0.77 \\
1000 & 0.86 & $\mathbf{1 . 0 0}$ & 0.74 & 0.96 & 0.02 & 0.78
\end{tabular}

Table 5.5: Recall (He-based) in AIES

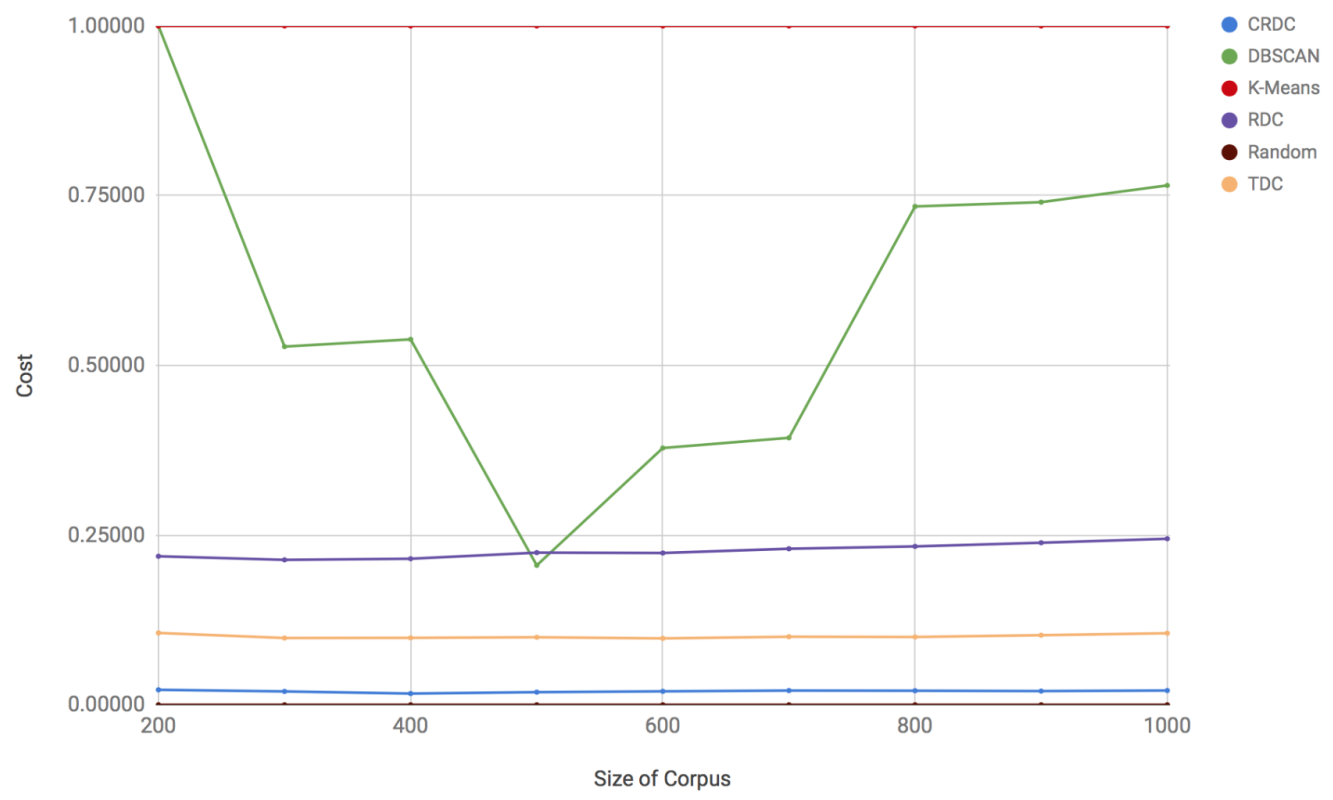

Figure 5.19: Cost (JS-based) in AIES

Unlike AIES, topic distributions have been randomly generated which imply that the similarity values are not so high: $\min =0.06$, mean $=0.18$ and $\max =0.61$. Following the same criteria than before (Section 5.2.2.2), the similarity threshold is now fixed to 0.34 (Figure 5.23). Results in terms of effectiveness (Figure 5.24) show a poor performance of the RDC and CRDC algorithms. The reason is that both are based on the fact that the highest weighted topics are shared between similar distributions. 


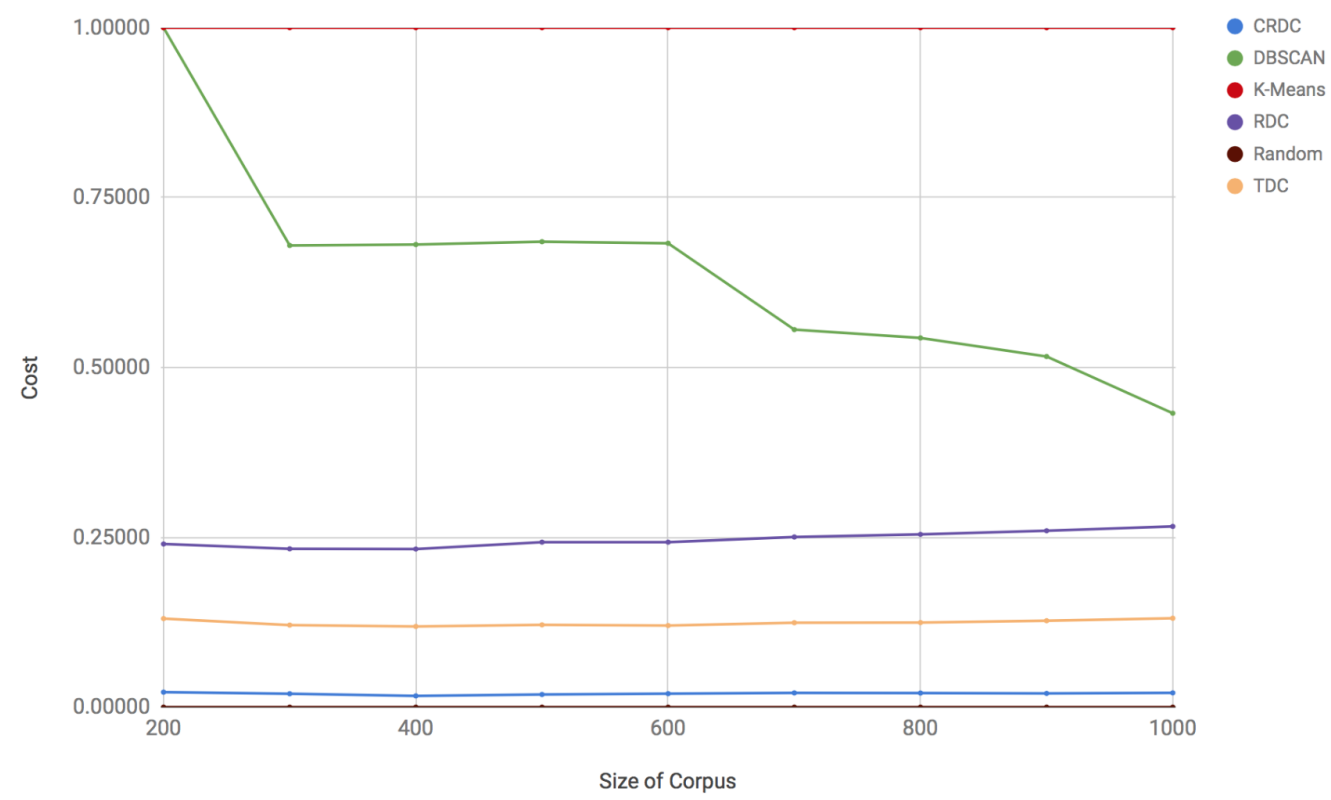

Figure 5.20: Cost (He-based) in AIES

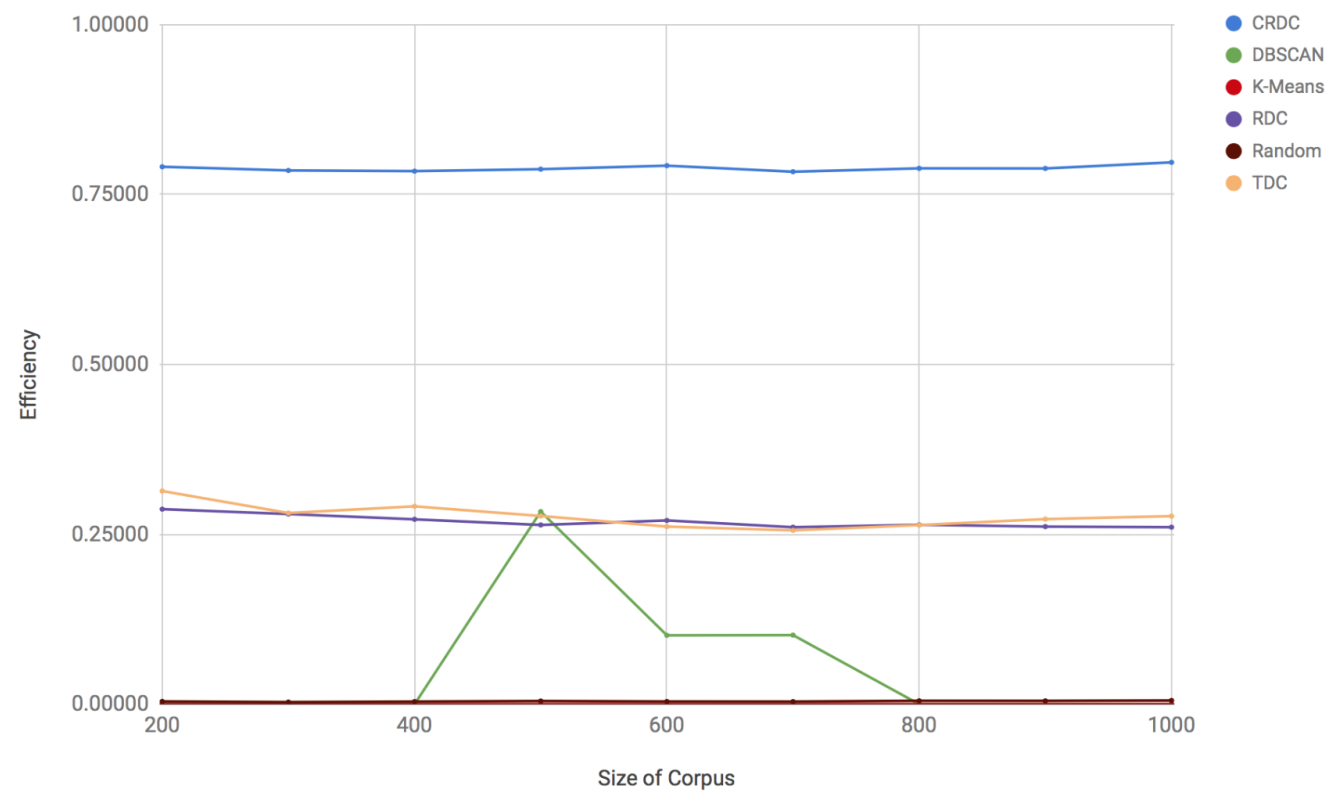

Figure 5.21: Efficiency (JS-based) in AIES

However, this condition is not satisfied when the similarity value between them is low.

To confirm this behavior, we created a third dataset (DRM2) with the same size 


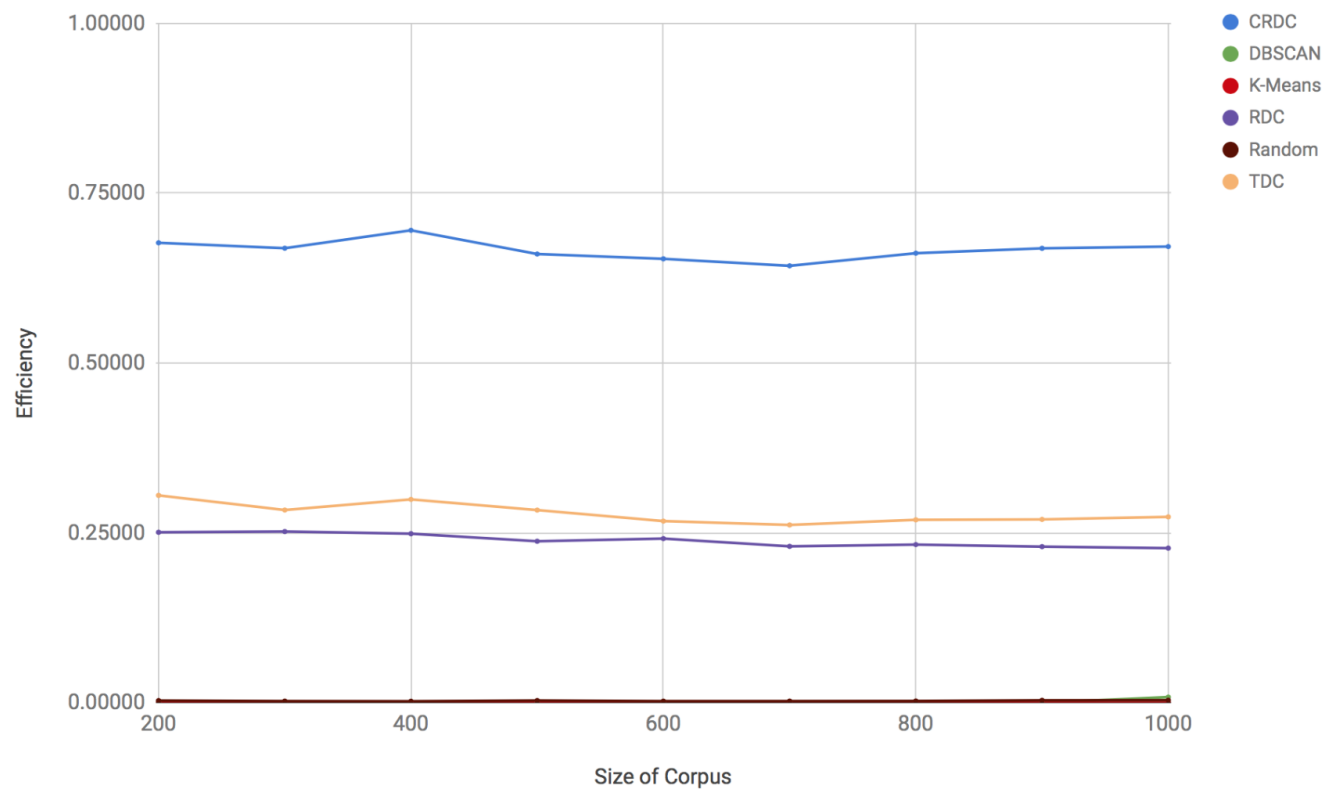

Figure 5.22: Efficiency (He-based) in AIES

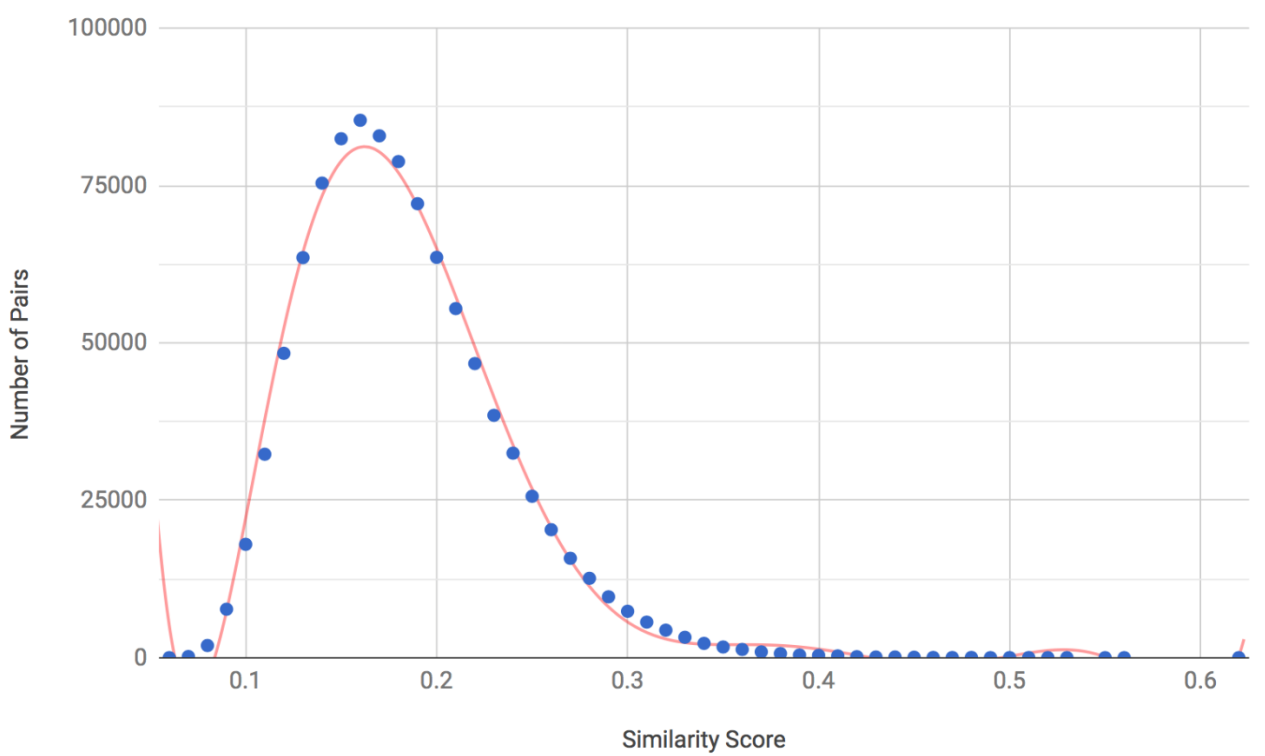

Figure 5.23: Similarity values grouped by frequency in DRM

but with only 4 dimensions ( 4 topics). The goal is to achieve more similar distributions than in DRM even though they are also randomly generated. Since the similarity values range from $\min =0.04$, mean $=0.34$ to $\max =0.99$, the similarity threshold 
is now fixed to 0.66 (more details in section 5.2.2.2). The results (Figure 5.25) show an improvement in the accuracy of both the RDC and CRDC algorithms. Although scores are still not as high as for the AIES dataset, the increase compared to the DRM dataset shows that their precision and recall improve when the similarity threshold is higher. On the other hand, both the DBSCAN and TDC algorithms show similar behavior in both datasets, which means that their performance is not affected by the similarity threshold.

\subsubsection{Conclusion}

Processing a continuously growing collection of human generated documents requires techniques that divide the space into smaller regions containing potentially similar documents. Some algorithms in the literature tackle this problem from an unsupervised point of view, but they incur in high temporal costs and may not be suited for the domain being studied.

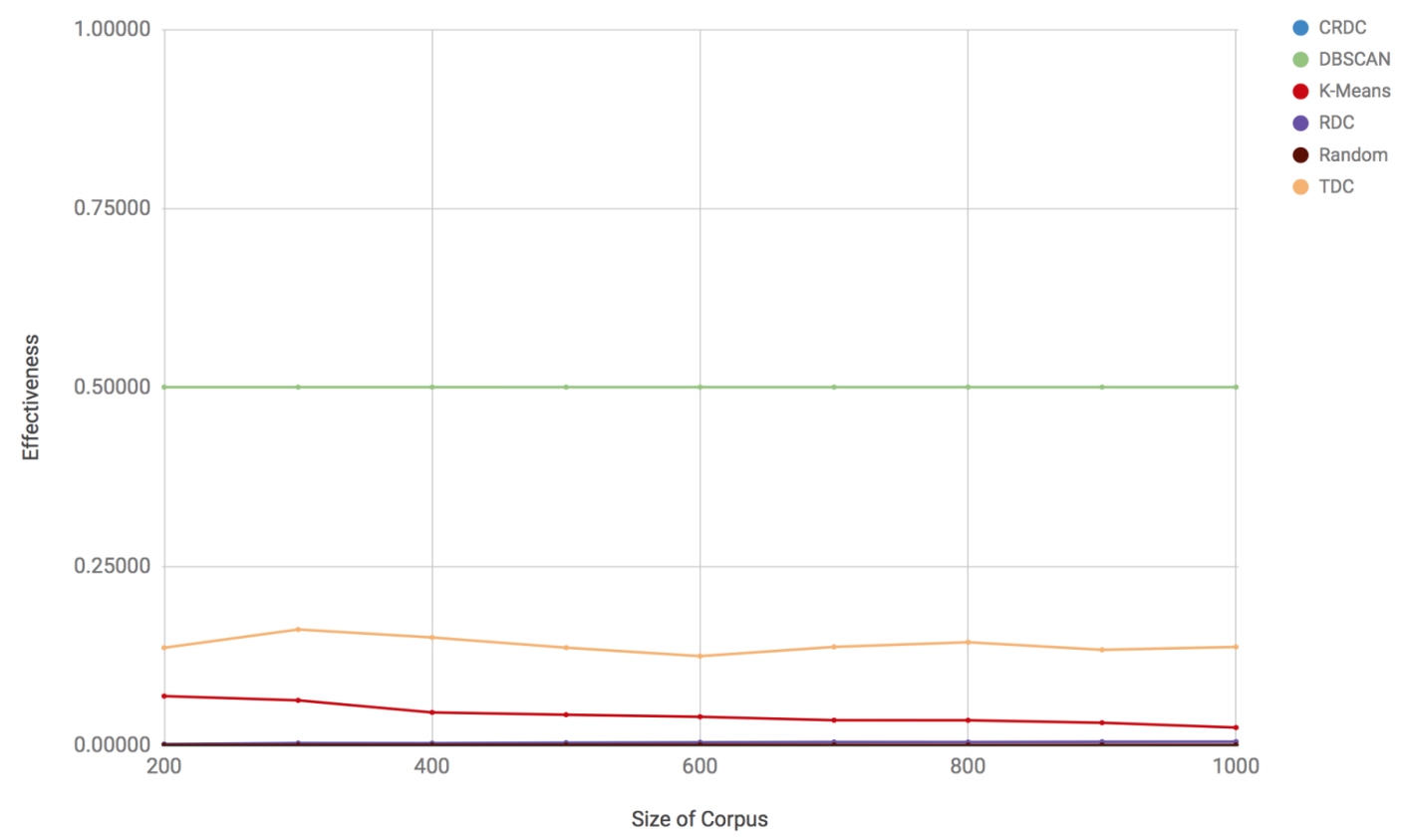

Figure 5.24: Effectiveness (JS based) in DRM

Three novel unsupervised clustering algorithms, TDC, RDC and $C R D C$, are described in this section relying on the distributions inferred from a topic modeling algorithm (LDA). They are presented as a means to identify a smaller set of documents 


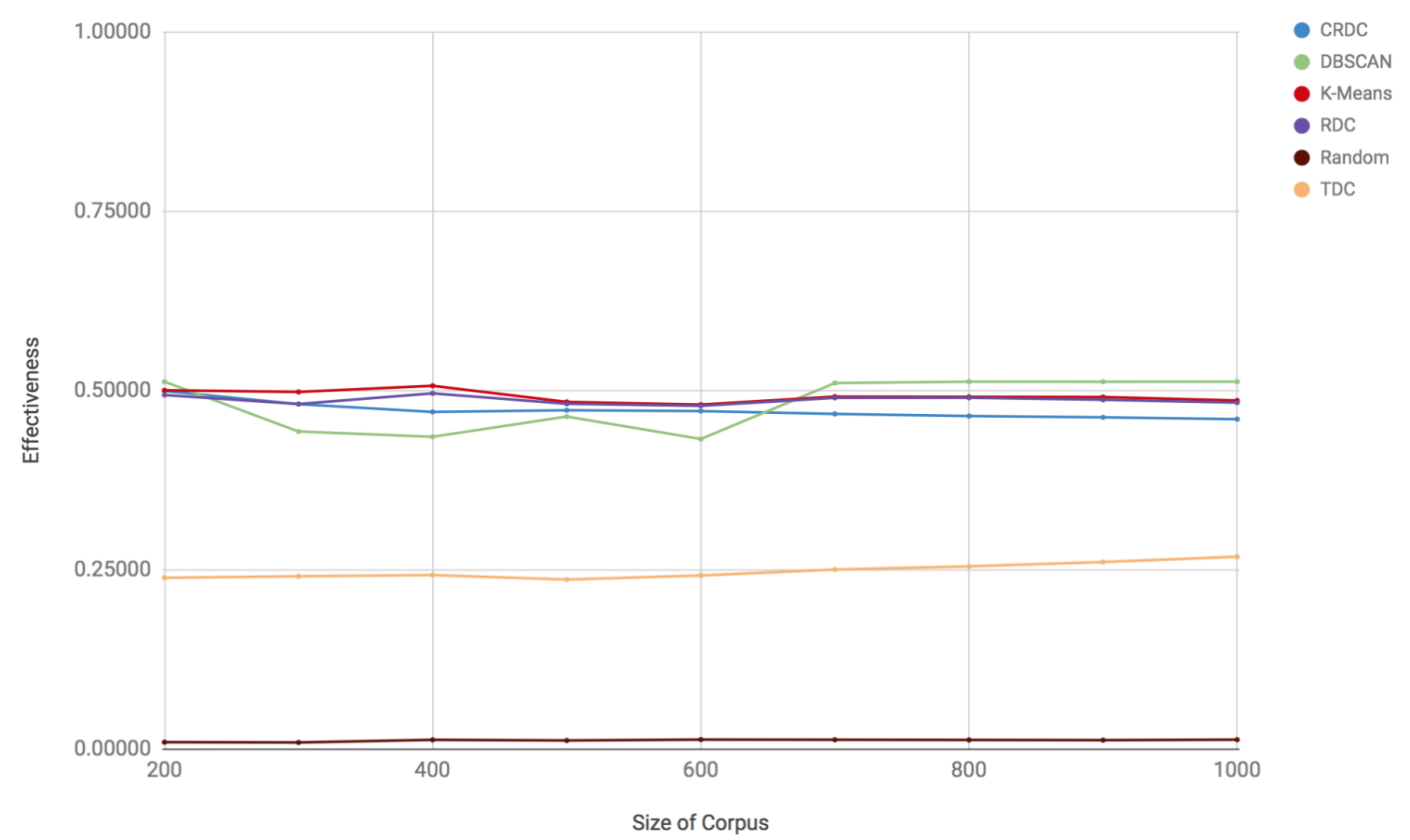

Figure 5.25: Effectiveness (JS based) in DRM2

where only the similarity function has to be computed. They leverage on the particular behavior of Dirichlet distributions describing topic distributions, where the highest weighted topics have a high influence on the rest of topics. This also means that given a topic distribution, the relations between their topic weights such as order or trends between them, are more important than the density values.

Although we initially thought that using only a fixed number of topics with higher weights of a topic distribution $(R D C)$, or taking into account only the trend changes between the weights of consecutive topics $(T D C)$, could be enough to classify similar topic distributions, the results obtained have shown that these properties are not sufficient. Results in terms of efficiency, effectiveness and cost have been shown comparing the proposed algorithms with existing centroid-based and density-based clustering techniques. They reveal that obtaining the most representative topics of a topic distribution by comparing the sum of their weights with respect to the rest $(C R D C)$ is a promising approach, which improves the efficiency obtained by other centroid-based and densitybased approaches. While $K$-Means takes $O\left(n^{k} * \log n\right)$ and $D B S C A N$ takes $O(n * \log n)$ time to classify $n$ documents in a collection, the proposed algorithms only take lin- 
ear time $(O(n))$ because they do not require any other data except their own topic distribution to assign it to a cluster.

\subsection{Summary}

In Section 5.1, we have analyzed the representativeness of topics to describe texts, using the particular case of scientific articles. Our experiment with a corpus of research papers published in the Advances in Engineering Software (AIES) journal shows that abstracts are not sufficiently representative to describe, by means of topics, the content of a paper. This behavior suggests that texts with greater vocabulary that emphasize key terms through repetition, favor topic-based representation. Therefore, if we want to construct a system that relates scientific articles, it would be better to use full texts rather than abstracts, as it is done by many more traditional techniques. This has a higher computational cost, for which systems like librAIry behave sufficiently well.

Taking into account the relevance of topics to describe texts, we analyze in Section 5.2 the behavior of topic distributions to calculate distances between documents using topic models with different dimensions. By using clustering techniques at the topic level, the most representative topics of a topic distribution are identified regardless of the number of dimensions that the model has. A topic-based representation is then proposed that covers the third research objective of this thesis (R03, define annotations based on topics that enable a semantic-aware exploration of the knowledge inside a corpus).

A new distance metric is also proposed that takes advantage of such representation to compare documents. Its performance is analyzed by automatically clustering the JRC-Acquis corpus according to EUROVOC categories. Tables 5.3, 5.2, 5.4 and 5.5 show results with high precision and recall in unsupervised classification tasks. This new way of relating documents from their most representative topics covers the fourth research objective of this thesis (R04, define a metric based on topic annotations that compares documents and facilitates their interpretation).

In order to perform the experiments, both the representation based on the most relevant topics and the distance metric based on these representations have been implemented in librAIry. This partially covers the third and fourth technical objectives 
(T03, integrate the annotation method base on topic hierarchies into the topic model service) (T04, create a system capable of finding similar document automatically). 


\section{Chapter 6}

\section{Large-scale Monolingual Document Similarity}

As we showed in Section 5.2, grouping topics by a cumulative ranking is a useful mechanism for simplifying representations based on topic distributions. Relevant topics emerge as those whose accumulated weight exceeds a threshold, after ordering all topics and starting from the top. This technique has shown a promising performance to cluster documents ( Section 5.2.2.5) and indicates that related documents share the most relevant topics. However, the approach still has limitations: it depends on the manual tuning of a parameter, the threshold that help us identifying relevant documents; and it does not measure degrees of similarity since it only establishes whether or not two documents are related. As shown in Chapter 3 , we hypothesize whether is possible to find relevant documents with similar topic distributions without calculating all pairwise comparisons and without discarding the notion of topics from their representation. In this chapter we introduce the notion of relevance levels and present our approach to compare documents from huge collections through hierarchical representations of their topic distributions (Badenes-Olmedo et al., 2019b).

\subsection{Hashing Topic Distributions}

One of the greatest advantages of using Probabilistic Topic Models (PTM) in large document collections is the ability to represent documents as probability distributions over a finite number of topics, thereby mapping documents into a low-dimensional 
latent space (the K-dimensional probability simplex, where $\mathrm{K}$ is the number of topics). A document, represented as a point in this simplex, is considered to have a particular topic distribution. As seen in Section 5.2, the low-dimensional feature space created by topic models could also be suitable for document similarity tasks, especially on big real-world data sets, since topic distributions are continuous and not as sparse as discrete-term feature vectors and can be explained in terms of relevance.

Hashing methods transform the data points from the original feature space into a binary-code Hamming space, where the similarities in the original space are preserved. They can learn hash functions (data-dependent) or use projections (data-independent) from the training data (Wang et al., 2016). Data-independent methods unlike datadependent ones do not need to be re-calculated when data changes, i.e. adding or removing documents to the collection. Taking large-scale scenarios into account (e.g. Document clustering, Content-based Recommendation, Duplicate Detection), this is a key feature along with the ability to infer hash codes individually (for each document) rather than on a set of documents.

Data-independent hashing methods depend on two key elements: (1) data type and (2) distance metric. For vector-type data, as introduced in Section 2.2 , based on $l_{p}$ distance with $p \epsilon[0,2)$ lots of hashing methods have been proposed, such as p-stable Locality-Sensitive Hashing (LSH) (Datar et al., 2004), Leech lattice LSH (Andoni and Indyk, 2006), Spherical LSH (Terasawa and Tanaka, 2007), and Beyond LSH (Andoni et al., 2014). Based on the $\theta$ distance many methods have been developed such as Kernel LSH (Kulis and Grauman, 2012) and Hyperplane hashing (Vijayanarasimhan et al. 2014). But only few methods handle density metrics in a simplex space. A first approach transformed the $H e$ divergence into an Euclidean distance so that existing ANN techniques, such as LSH and k-d tree, could be applied (Krstovski et al., 2013). But this solution does not consider the special attributions of probability distributions, such as Non-negative and Sum-equal-one. More recently, a hashing schema (Mao et al. 2017) taking into account the symmetry has been proposed, non-negativity and triangle inequality features of the S2JSD metric for probability distributions. For set-type data, Jaccard Coefficient is the main metric used. Some examples are K-min Sketch (Li et al. 2012), Min-max hash (Ji et al., 2013), B-bit minwise hashing (Li and König, 2010) and Sim-min-hash (Zhao et al., 2013). 
All of them have demonstrated efficiency in the search for related documents, but none of them allows the search for documents (1) by thematic areas or (2) by similarity levels, nor do they offer (3) an explanation about the similarity obtained beyond the vectors used to calculate it. Binary-hash codes drop a very precious information: the topic relevance.

A new hierarchical set-type data is proposed (Figure 6.1). Each level of the hierarchy indicates the importance of the topic according to its distribution. Level 0 contains the topics with the highest score. Level 1 contains the topics with highest score once the first ones have been eliminated, and so on. From a vector of components, where each of the components is the score of topic $t$, a vector containing set of topics is proposed, where each of the dimensions means a topic relevance. Thus, for the topic distribution $q=[0.02,0.14,0.02,0.16,0.04,0.09,0.19,0.12,0.04,0.17]$, a hierarchical set of topics may be $h=\{(t 6),(t 9, t 3),(t 1)\}$. It means that topic $t 6(0.19)$ is the most relevant, then topics $t 9(0.17)$ and $t 3(0.16)$ and, finally, topic $t 1(0.14)$. This is just an example about the data structure that will support the different hashing strategies. In Section 6.1.3 some approaches to create hash codes based on this data structure are described.

\subsubsection{Hierarchical Data}

As seen in Section 2.2, a traditional approach to text representation usually requires encoding of documents into numerical vectors. Words are extracted from a corpus as feature candidates and based on a certain criterion they are assigned values to describe the documents: term-frequency, TF-IDF, information gain, and chi-square are typical measures. But this causes two main problems: huge number of dimensions and sparse distribution. The use of topics as feature space has been extended to mapping documents into low-dimensional vectors. However, as shown in Figure 2.3, the distance metrics based on probability densities vary according to the dimensions of the model and reveal the difficulty of calculating the similarity values using the vectors with the topic distributions.

Domain-specific features such as vocabulary, writing style, or speech type, have a major influence on the topic models, but not in the hashing algorithms described in this section. The methods for creating hash codes are agnostic of these particularities since they are only based on the topic distributions generated by the models. 


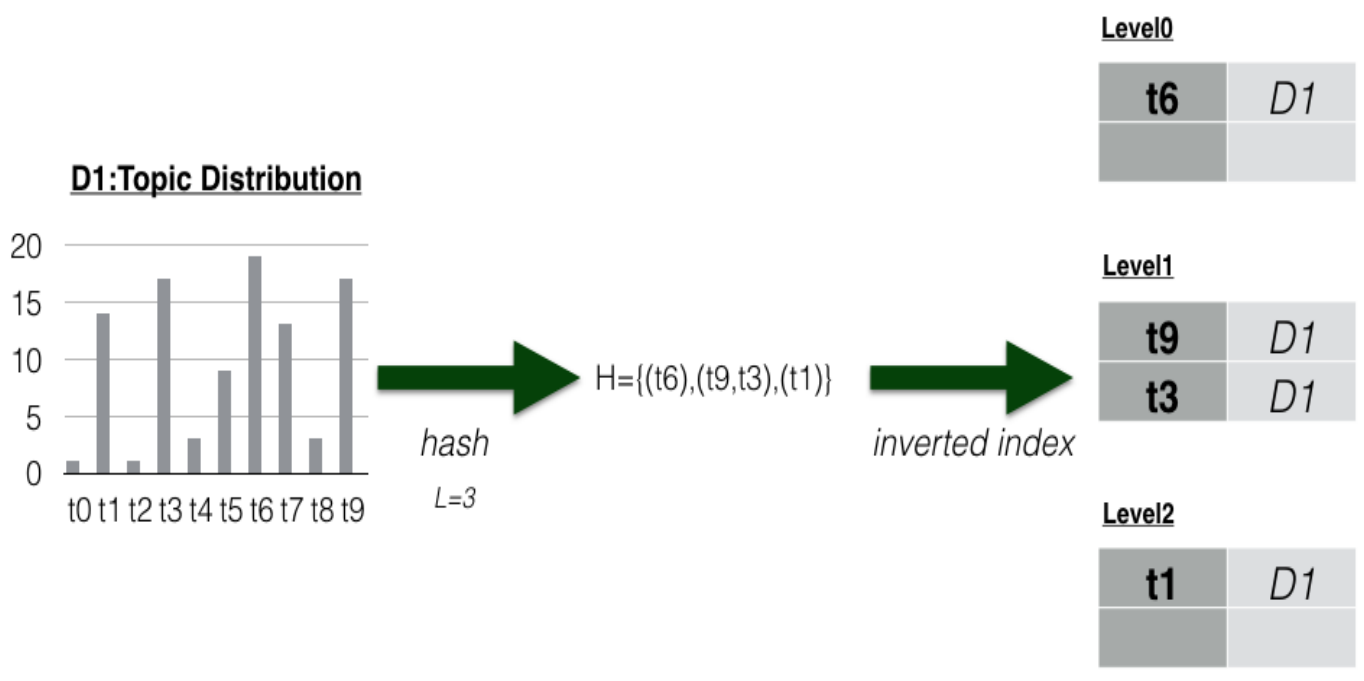

Figure 6.1: Hash method based on hierarchical set of topics from a given topic distribution

\subsubsection{Distance Metric}

Since documents are described by set-type data, the proposed distance metric is based on the Jaccard coefficient. This metric computes the similarity of sets by looking at the relative size of their intersection as follows:

$$
J(A, B)=\frac{|A \cap B|}{|A \cup B|}
$$

where $\mathrm{A}$ and $\mathrm{B}$ are set of topics.

More specifically, $d_{J}$ is based on the Jaccard distance, which is obtained by subtracting the Jaccard coefficient $J$ from 1 :

$$
d_{J}(A, B)=1-J(A, B)
$$

The proposed distance measure $d_{H}$ used to compare hash codes created from set of topics is the sum of the Jaccard distances $d_{j}$ for each hierarchy level, i.e. for each set 
of topics:

$$
d_{H}\left(H_{1}, H_{2}\right)=\sum_{l=1}^{L}\left(d_{J}\left(H_{1}\left(x_{l}\right), H_{2}\left(x_{l}\right)\right)\right)
$$

where $H_{1}$ and $H_{2}$ are hash codes, $H_{1}\left(x_{l}\right)$ and $H_{2}\left(x_{l}\right)$ are the set of topics up to level $l$ for each hash code $H$ and $L$ is the maximum hierarchy level. A corner case is $L=T$, where $T$ is the number of topics in the model.

\subsubsection{Hash Function}

The hash function clusters topics based on relevance levels. Three approaches are proposed depending on the criteria used to group topics: threshold-based, centroidbased and density-based.

\subsubsection{Threshold-based Hierarchical Hashing Method}

This approach is just an initial and naive way of grouping topics by threshold values into each relevance level. They can be manually defined or automatically generated by thresholds dividing the topic distributions as follows:

$$
t h_{i n c}=\frac{1}{(L+1) \cdot T}
$$

where $L$ is the number of hierarchy levels, and $T$ the number of topics.

If $L=3$ and $T=10$ for a topic distribution $t d$ defined as follows:

$$
\begin{gathered}
t d=[0.017,0.141,0.010,0.172,0.030, \\
0.090,0.199,0.133,0.031,0.171]
\end{gathered}
$$

Then, a threshold-based hierarchical hash $H_{T}$, with an automatically created threshold defined by equation 6.4 is equals to $H_{T}=\{(t 1, t 3, t 5, t 6, t 7, t 9),(),(t 4, t 8)\}$ with $t h_{\text {inc }}=0.025($ Fig 6.2) .

\subsubsection{Centroid-based Hierarchical Hashing Method}

This approach assumes topic distributions can be partitioned into $k$ clusters where each topic belongs to the cluster with the nearest mean score. It is based on the k-Means clustering algorithm, where $k$ is obtained by adding 1 to the number of hierarchy levels. Unlike the previous method, threshold values used to define the hierarchy levels may 


\section{Threshold-based Hashing}

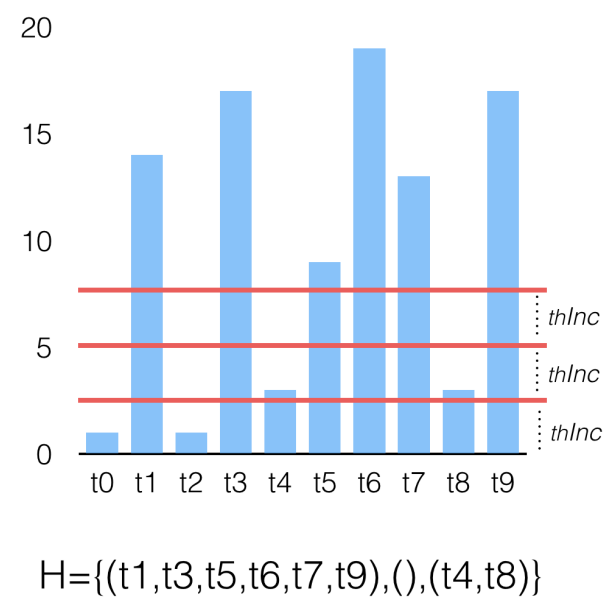

Figure 6.2: Threshold-based Hierarchical Hash $(\mathrm{L}=3)$

vary between documents, i.e. for each topic distribution, since they are calculated for each distribution separately.

Following the previous example, if $L=3$ and $T=10$ for a topic distribution $t d$ defined in equation 6.5, then a centroid-based hieararchical hash $H_{C}$ equals to $H_{C}=$ $\{(t 6),(t 9, t 7, t 3, t 1),(t 5)\}($ Fig 6.3) .

\subsubsection{Density-based Hierarchical Hashing Method}

This approach also considers relative hierarchical thresholds for each relevance level. Now, a topic distribution is described by points in a single dimension. In this space, topics closely packed together are grouped together. This approach does not require a fixed number of groups. It only requires a maximum distance (eps) to consider two points close and grouped together. This value can be estimated from the own distribution of topics (e.g. variance).

Following the above example, if $L=3$ and $t d$ is the topic distribution defined in equation 6.5. then a density-based hierarchical hash $H_{D}$ is equals to $H_{D}=\{(t 6),(t 9, t 3),(t 1)\}$ when eps equals to the variance of the topic distribution (Fig 6.4). 


\section{Centroid-based Hashing}

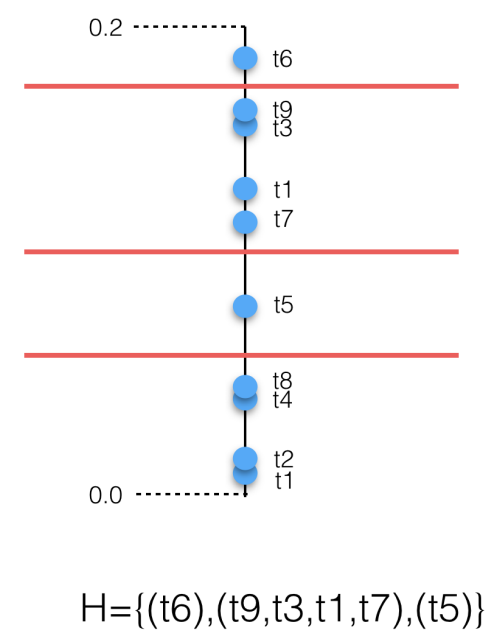

Figure 6.3: Centroid-based Hierarchical Hash $(\mathrm{L}=3)$

\subsubsection{Online-mode Hashing}

Hashing methods are batch-mode learning models that require huge data for learning an optimal model and cannot handle unseen data. Recent work address online mode by learning algorithms (Huang et al., 2018) that get hashing model accommodate to each new pair of data. But these approaches require the hashing model to be updated during each round based on the new pairs of data.

Our methods rely on topic models to build hash codes. These models do not require to be updated to make inferences about data not seen during training. In this way, the proposed hashing algorithms can work on large-scale and real-time data, as the size and the novelty of the collection does not influence the annotation process.

\subsection{Evaluation}

As mentioned in Section 2.4.2, it is difficult to interpret the similarity score calculated by metrics in a probability space. Since all of them are based on adding the distance between each dimension of the model (eq. 2.1, 2.2 and 2.4), distributions that share a fair amount of the less representative topics may still get higher similarity values than those that share the most representative ones specially if the model has a high number of dimensions. 


\section{Density-based Hashing}

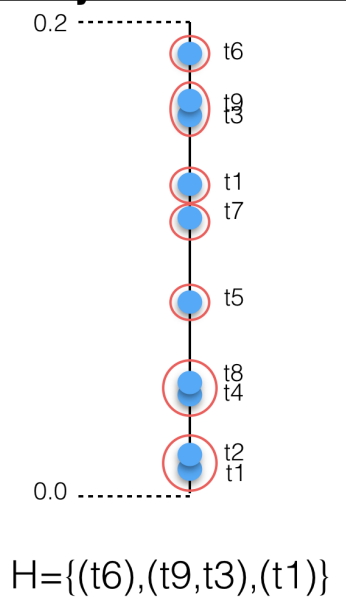

Figure 6.4: Density-based Hierarchical Hash $(\mathrm{L}=3)$

Figures 6.5 and 6.6 show overlapped topic distributions of two pairs of documents. In the first case (fig 6.5), none of the most representative topics of each document is shared between them. However, the similarity score calculated from divergence-based metrics (eq 2.2) is higher than in the second case (fig 6.6), where the most representative topic is shared (topic 26). This behavior is due to the sum of the distances between the less representative topics (i.e. topics with a low weight value) being greater than the sum of the distances between the most representative ones (i.e. topic with a high weight value). In high-dimensional models, that sum may be more representative than the one obtained with the most relevant topics, which are fewer in number than the less relevant ones.

The following experiments aim to validate that hash codes based on hierarchical set of topics not only make it possible to search for related documents with high accuracy, but also to extend queries with new restrictions and to offer information that helps explaining why two documents are related.

\subsubsection{Datasets and Evaluation Metrics}

Three dataset: ${ }^{72}$ are used to validate the proposed approach. The OPEN-RESEARCH ${ }^{73}$ dataset consist of 500k research papers in Computer Science, Neuroscience, and Biomed-

\footnotetext{
${ }^{72}$ https://doi.org/10.5281/zenodo.3465855

${ }^{73}$ https://labs.semanticscholar.org/corpus/
} 


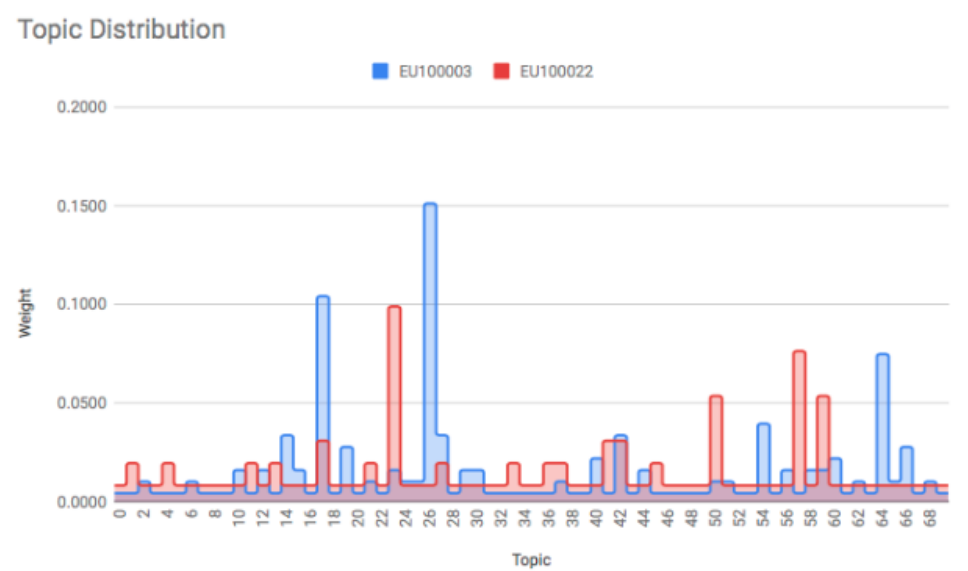

Figure 6.5: Topic Distribution of two documents with similarity score, based on JS, equals to 0.74

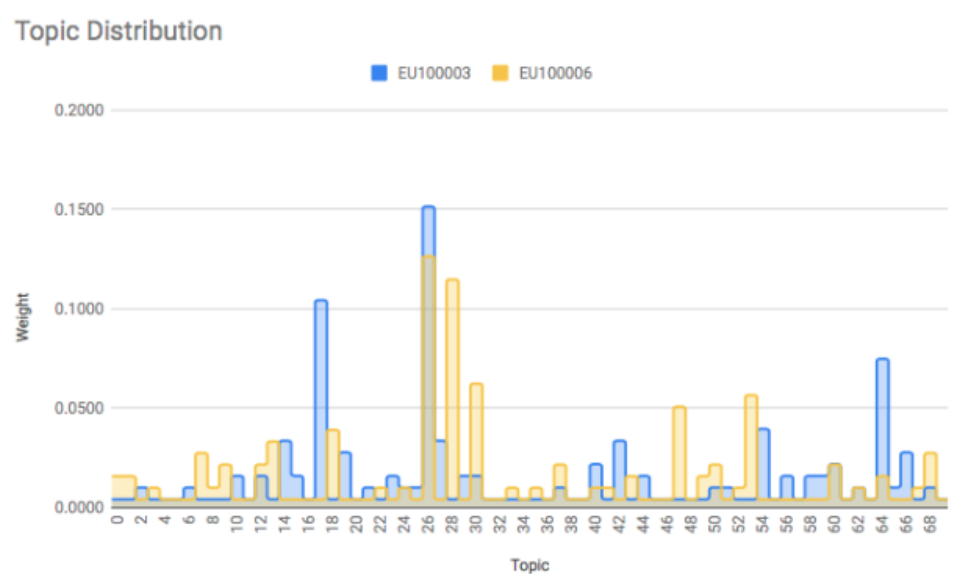

Figure 6.6: Topic Distribution of two documents with similarity score, based on JS, equals to 0.71

ical randomly selected from the Open Research Corpus (Ammar et al., 2018). The CORDIS 74 dataset contains 100k documents describing research and innovation projects funded by the European Union under a framework programme since 1990. The PATENTS dataset consists of $1 \mathrm{M}$ patents randomly selected from the USPTO 75 collection. For each dataset, documents are mapped to two latent topic spaces with different dimensions using LDA. We perform parameter estimation using collapsed Gibbs sampling for

\footnotetext{
${ }^{74}$ https://data.europa.eu/euodp/data/dataset/cordisref-data

${ }^{75}$ https://www.uspto.gov/learning-and-resources/ip-policy/economic-research/research-datasets
} 
LDA (Griffiths and Steyvers, 2004) from our librAIry framework. The number of topics varies to study their influence on the performance of the algorithm (i.e. CORDIS-70 indicates a latent space created with 70 topics).

Experiments use JS divergence as an information-theoretically motivated metric in the probabilistic space created by topic models. Since it is a smoothed and symmetric alternative to the KL divergence, which is a standard measure for comparing distributions (Cha, 2007), it has been extensively used as state-of-the-art metric over topic distributions in literature (Aletras et al., 2017; Mao et al., 2017, Towne et al., 2016). Our upper bound is created from the brute-force comparison of the reference documents with all documents in the collection to obtain the list of related documents.

In this scenario the goal is to minimize the accuracy loss introduced by hashing algorithms. Since this is a large-scale problem and an accuracy-oriented task, recall is not a good measure to be considered and precision is only relevant for sets much smaller than the total size of data (between 3-5 candidates).

All the experimental results are averaged over random training/set partitions. For each topic space, 100 documents are selected as references, and the remaining documents as search space. As noted above, only p@5 will be used to report the results of the experiments.

\subsubsection{Retrieving Related Documents}

It is challenging to create an exhaustive gold standard, given the significant amount of human labour that is required to get a comprehensive view of the subjects being covered in it. In order to overcome this problem, the list of related documents to a given one is obtained after comparing the document with all the documents of the repository and sorting the result. We have observed that different distance functions perform similarly in this scenario (Fig. 2.3), so we have decided to use only the JS divergence (eq. 2.2) in our experiments.

Only the top $\mathrm{N}$ documents obtained from this method are used as reference set to measure the performance of the algorithms proposed in this paper. The value of $\mathrm{N}$ is equals to $0.5 \%$ of the corpus size (i.e. if the corpus size is equal to 1000 elements, only the top 5 most related documents are considered relevant for a given document). This value has been considered after reviewing datasets used in similar experiments (Krstovski et al., 2013; Mao et al., 2017). In those experiments, the reference data is 


\begin{tabular}{c|cc||cc||cr}
\multicolumn{8}{c}{ OPEN-RES-100 (p@5) } \\
\hline \multirow{2}{*}{ LEVEL } & \multicolumn{2}{|c}{ THHM } & \multicolumn{2}{c}{ CHHM } & \multicolumn{2}{c}{ DHHM } \\
& mean & median & mean & median & mean & median \\
\hline \multirow{2}{*}{2} & 0.22 & 0.20 & $\mathbf{0 . 8 6}$ & 1.00 & 0.66 & 0.80 \\
3 & 0.23 & 0.20 & $\mathbf{0 . 8 7}$ & 1.00 & 0.81 & 1.00 \\
4 & 0.27 & 0.20 & $\mathbf{0 . 8 9}$ & 1.00 & 0.86 & 1.00 \\
5 & 0.27 & 0.20 & $\mathbf{0 . 9 2}$ & 1.00 & 0.89 & 1.00 \\
6 & 0.27 & 0.20 & $\mathbf{0 . 9 4}$ & 1.00 & 0.92 & 1.00 \\
\hline
\end{tabular}

Table 6.1: Precision at 5 (mean and median) of threshold-based (THHM), centroid-based (CHHM) and density-based (DHHM) hierarchical hashing methods on Open Research dataset using a model with 100 topics. LEVEL column indicates the number of hierarchies used.

OPEN-RES-500 (p@5)

\begin{tabular}{c|rr||rr||rr}
\hline \multirow{2}{*}{ LEVEL } & \multicolumn{2}{|c}{ THHM } & \multicolumn{2}{c}{ CHHM } & \multicolumn{2}{c}{ DHHM } \\
& mean & median & mean & median & mean & median \\
\hline 2 & 0.23 & 0.20 & $\mathbf{0 . 7 6}$ & 0.80 & 0.67 & 0.80 \\
3 & 0.24 & 0.20 & $\mathbf{0 . 8 0}$ & 1.00 & 0.71 & 0.80 \\
4 & 0.25 & 0.20 & $\mathbf{0 . 8 3}$ & 1.00 & 0.74 & 0.80 \\
5 & 0.25 & 0.20 & $\mathbf{0 . 8 6}$ & 1.00 & 0.81 & 1.00 \\
6 & 0.24 & 0.20 & $\mathbf{0 . 8 9}$ & 1.00 & 0.86 & 1.00 \\
\hline
\end{tabular}

Table 6.2: Precision at 5 (mean and median) of threshold-based (THHM), centroid-based (CHHM) and density-based (DHHM) hierarchical hashing methods on Open Research dataset using a model with 500 topics. LEVEL column indicates the number of hierarchies used.

obtained from existing categories, and the minimum average between corpus size and categorized documents is around $0.5 \%$.

Once the reference list of documents related to a given one is defined, the most relevant documents through the proposed methods (i.e. threshold-based hierarchical hashing method (thhm), centroid-based hierarchical hashing method (chhm) and density-based hierarchical hashing method (dhhm)) are also obtained. An inverted index has been implemented by using Apache Lucent ${ }^{76}$ as document repository. The source code of both the algorithms and tests is publicly available 77 .

Let's look at an example to better understand the procedure. We want to measure the accuracy and data size ratio used to identify the top5 related documents to a new

\footnotetext{
${ }^{76} \mathrm{http}: / /$ lucene.apache.org

${ }^{77}$ https://doi.org/10.5281/zenodo.3465855
} 
CORDIS-70 (p@5)

\begin{tabular}{c|cc||cr||rr}
\hline \multirow{2}{*}{ LEVEL } & \multicolumn{2}{|c}{ THHM } & \multicolumn{2}{c}{ CHHM } & \multicolumn{2}{c}{ DHHM } \\
& mean & median & mean & median & mean & median \\
\hline 2 & 0.18 & 0.20 & $\mathbf{0 . 9 2}$ & 1.00 & 0.66 & 0.70 \\
3 & 0.20 & 0.20 & $\mathbf{0 . 9 2}$ & 1.00 & 0.80 & 0.80 \\
4 & 0.22 & 0.20 & $\mathbf{0 . 9 4}$ & 1.00 & 0.86 & 1.00 \\
5 & 0.23 & 0.20 & $\mathbf{0 . 9 1}$ & 1.00 & 0.89 & 1.00 \\
6 & 0.19 & 0.20 & $\mathbf{0 . 9 2}$ & 1.00 & 0.91 & 1.00 \\
\hline
\end{tabular}

Table 6.3: Precision at 5 (mean and median) of threshold-based (THHM), centroid-based (CHHM) and density-based (DHHM) hierarchical hashing methods on CORDIS dataset using a model with 70 topics. LEVEL column indicates the number of hierarchies used.

\begin{tabular}{c|cc||cc||cr}
\multicolumn{8}{c}{ CORDIS-150 (p@5) } \\
\hline \multirow{2}{*}{ LEVEL } & \multicolumn{2}{|c}{ THHM } & \multicolumn{2}{c}{ CHHM } & \multicolumn{2}{c}{ DHHM } \\
& mean & median & mean & median & mean & median \\
\hline \multirow{2}{*}{2} & 0.19 & 0.20 & $\mathbf{0 . 8 8}$ & 1.00 & 0.78 & 0.80 \\
3 & 0.19 & 0.20 & $\mathbf{0 . 9 2}$ & 1.00 & 0.80 & 1.00 \\
4 & 0.25 & 0.20 & $\mathbf{0 . 9 1}$ & 1.00 & 0.82 & 1.00 \\
5 & 0.25 & 0.20 & $\mathbf{0 . 9 1}$ & 1.00 & 0.83 & 1.00 \\
6 & 0.27 & 0.20 & $\mathbf{0 . 9 1}$ & 1.00 & 0.86 & 1.00 \\
\hline
\end{tabular}

Table 6.4: Precision at 5 (mean and median) of threshold-based (THHM), centroid-based (CHHM) and density-based (DHHM) hierarchical hashing methods on CORDIS dataset using a model with 150 topics. LEVEL column indicates the number of hierarchies used. 
document $d_{1}$ from a corpus of 1000 documents. The similarity between $d 1$ and all the documents in the corpus is calculated based on JS divergence. The top50 (0.5\%) documents with the highest values will be the set of documents considered as similar to $d_{1}$. As we are going to use an ANN-based approach, we need the hash expressions of all documents to measure similarity. The data structure proposed in this chapter is a hierarchy of sets of topics, so that the most related documents are those that share most of the topics at the highest levels of the hierarchy.

The representational model for this example only considers 8 topics, that is, a document is described by a vector with 8 dimensions where each dimension corresponds to a topic (i.e $[t 0, t 1, t 2, t 3, t 4, t 5, t 6, t 7])$ and its value will be the weight of that topic in the document, for example $d_{1}=[0.18,0.15,0.2,0.05,0.14,0.11,0.09$,

0.08]. The hierarchy level $(L)$ will be equal to 2, i.e. the hash expression has two hierarchical sets of topics: $h=\left\{h_{0}, h_{1}\right\}$.

According to methods described at Section 6.1.3, there are 3 ways to create the hierarchical hash codes for documents:

1. threshold-based $(t h h m): 2$ thresholds are defined as described in section 6.1.3.1, for example 0.15 and $0.1 . h_{0}$ includes the topics with a weight greater than 0.15 , and $h_{1}$ the remaining topics with a weight greater than 0.1 . Then $h_{0}=\{t 0, t 1, t 2\}$ and $h_{1}=\{t 4, t 5\}$. Based on the hash expression $h=\{(t 0, t 1, t 2),(t 4, t 5)\}$, the documents that share more topics in those levels (i.e $h 0=(t 0$ OR $t 1$ OR $t 2)$, $h 1=(t 4$ OR $t 5))$ or in other levels but with less relevance are ordered. Since there are many topics in the expression, potentially many documents are related when sharing at least one of them. This increases the data ratio. Accuracy is also affected, as the algorithm is not able to bring under the same bucket related documents. In short, the hash expression is not representative of the document, for the given exploratory task.

2. centroid-based $(\operatorname{chhm})$ : sets of topics are created using a clustering algorithm based on centroids as described in section 6.1.3.2. The cardinalities of the hierarchical groups are generally more uniform with this method. Since $k=L+1=3$ in this example, $h_{0}=\{t 0, t 2\}$ and $h_{1}=\{t 1, t 4\}$. The number of representative topics at each level of the hierarchy is usually lower, and this causes the data ratio used to discover related documents to decrease as well. This approach increases 


\begin{tabular}{c|cc||cr||rr}
\hline \multicolumn{1}{c}{ PATENTS-250 (p@5) } \\
\hline \multirow{2}{*}{ LEVEL } & \multicolumn{2}{|c}{ THHM } & \multicolumn{2}{c}{ CHHM } & \multicolumn{2}{c}{ DHHM } \\
& mean & median & mean & median & mean & median \\
\hline \multirow{2}{*}{2} & 0.03 & 0.00 & $\mathbf{0 . 7 1}$ & 0.80 & 0.67 & 0.80 \\
3 & 0.08 & 0.00 & $\mathbf{0 . 9 1}$ & 1.00 & 0.90 & 1.00 \\
4 & 0.11 & 0.00 & $\mathbf{0 . 9 5}$ & 1.00 & $\mathbf{0 . 9 5}$ & 1.00 \\
5 & 0.12 & 0.00 & 0.95 & 1.00 & $\mathbf{0 . 9 6}$ & 1.00 \\
6 & 0.11 & 0.00 & $\mathbf{0 . 9 7}$ & 1.00 & $\mathbf{0 . 9 7}$ & 1.00 \\
\hline
\end{tabular}

Table 6.5: Precision at 5 (mean and median) of threshold-based (THHM), centroid-based (CHHM) and density-based (DHHM) hierarchical hashing methods on Patents dataset using a model with 250 topics. LEVEL column indicates the number of hierarchies used.

\begin{tabular}{c|rr||rr||rr}
\multicolumn{7}{c}{ PATENTS-750 (p@5) } \\
\hline \multirow{2}{*}{ LEVEL } & \multicolumn{2}{|c}{ THHM } & \multicolumn{2}{c}{ CHHM } & \multicolumn{2}{c}{ DHHM } \\
& mean & median & mean & median & mean & median \\
\hline \multirow{2}{*}{2} & 0.02 & 0.00 & $\mathbf{0 . 7 7}$ & 0.80 & 0.76 & 0.80 \\
3 & 0.04 & 0.00 & 0.94 & 1.00 & $\mathbf{0 . 9 5}$ & 1.00 \\
4 & 0.06 & 0.00 & $\mathbf{0 . 9 7}$ & 1.00 & $\mathbf{0 . 9 7}$ & 1.00 \\
5 & 0.08 & 0.00 & $\mathbf{0 . 9 7}$ & 1.00 & $\mathbf{0 . 9 7}$ & 1.00 \\
6 & 0.06 & 0.00 & $\mathbf{0 . 9 7}$ & 1.00 & $\mathbf{0 . 9 7}$ & 1.00 \\
\hline
\end{tabular}

Table 6.6: Precision at 5 (mean and median) of threshold-based (THHM), centroid-based (CHHM) and density-based (DHHM) hierarchical hashing methods on Patents dataset using a model with 750 topics. LEVEL column indicates the number of hierarchies used.

the precision because now the hierarchy is more selective to distinguish related documents. However, the size of region of related candidates is still high.

3. density-based $(d h h m)$ : now the clustering algorithm is based on how dense certain regions in the topic relevance dimensions are as described in section 6.1.3.3. It can group topics that have unbalanced distributions and, therefore, generates more discriminating hash expressions than with the previous algorithm. In the example, we would have a hash expression like this: $h_{0}=\{t 2\}$ and $h_{1}=\{t 0\}$. This significantly reduces the data ratio used to discover related documents and does not excessively penalize accuracy. Obviously, increasing $L$ (i.e. number of hierarchies) increases precision, but with $L>3$ that gain is not so significant.

As it can be seen in tables 6.1 to 6.6, the mean and median of precision are calculated to compare the performance of the methods. In this assessment environment, the variance is not robust-enough because score values don't follow a normal distribution. 
OPEN-RES-100 (data-ratio)

\begin{tabular}{c|rr||rr||rr}
\hline \multirow{2}{*}{ LEVEL } & \multicolumn{2}{|c}{ THHM } & \multicolumn{2}{c}{ CHHM } & \multicolumn{2}{c}{ DHHM } \\
& mean & median & mean & median & mean & median \\
\hline \multirow{2}{*}{2} & 99.8 & 99.9 & 45.2 & 45.9 & $\mathbf{4 . 9}$ & 2.5 \\
3 & 99.9 & 99.9 & 74.4 & 77.6 & $\mathbf{1 3 . 4}$ & 10.7 \\
4 & 99.9 & 99.9 & 87.4 & 90.2 & $\mathbf{2 7 . 2}$ & 22.8 \\
5 & 99.9 & 99.9 & 95.4 & 96.3 & $\mathbf{4 9 . 9}$ & 42.6 \\
6 & 99.9 & 99.9 & 97.9 & 98.7 & $\mathbf{7 2 . 2}$ & 65.8 \\
\hline
\end{tabular}

Table 6.7: Data size ratio used (mean and median) of threshold-based (THHM), centroidbased (CHHM) and density-based (DHHM) hierarchical hashing methods on Open Research dataset and 100 topics.

OPEN-RES-500 (data-ratio)

\begin{tabular}{c|rr||rr||rr}
\hline \multirow{2}{*}{ LEVEL } & \multicolumn{2}{|c}{ THHM } & \multicolumn{2}{c}{ CHHM } & \multicolumn{2}{c}{ DHHM } \\
& mean & median & mean & median & mean & median \\
\hline \multirow{2}{*}{2} & 95.9 & 96.3 & 22.2 & 22.1 & $\mathbf{1 . 4}$ & 0.3 \\
3 & 99.1 & 99.2 & 43.9 & 43.7 & $\mathbf{5 . 1}$ & 4.1 \\
4 & 99.6 & 99.6 & 57.1 & 57.3 & $\mathbf{1 1 . 7}$ & 10.3 \\
5 & 99.6 & 99.6 & 70.7 & 70.7 & $\mathbf{2 8 . 8}$ & 22.0 \\
6 & 99.9 & 99.9 & 81.5 & 80.6 & $\mathbf{5 0 . 3}$ & 40.1 \\
\hline
\end{tabular}

Table 6.8: Data size ratio used (mean and median) of threshold-based (THHM), centroidbased (CHHM) and density-based (DHHM) hierarchical hashing methods on Open Research dataset and 500 topics. 
CORDIS-70 (data-ratio)

\begin{tabular}{c|rr||rr||rr}
\hline \multirow{2}{*}{ LEVEL } & \multicolumn{2}{|c}{ THHM } & \multicolumn{2}{c}{ CHHM } & \multicolumn{2}{c}{ DHHM } \\
& mean & median & mean & median & mean & median \\
\hline \multirow{2}{*}{2} & 99.9 & 99.9 & 51.3 & 56.3 & $\mathbf{5 . 1}$ & 5.0 \\
3 & 99.9 & 99.9 & 84.8 & 89.5 & $\mathbf{1 0 . 5}$ & 10.6 \\
4 & 99.9 & 99.9 & 96.1 & 97.6 & $\mathbf{2 0 . 8}$ & 19.5 \\
5 & 99.9 & 99.9 & 98.9 & 99.4 & $\mathbf{3 5 . 0}$ & 32.7 \\
6 & 99.9 & 99.9 & 99.7 & 99.8 & $\mathbf{5 3 . 1}$ & 51.2 \\
\hline
\end{tabular}

Table 6.9: Data size ratio used (mean and median) of threshold-based (THHM), centroidbased (CHHM) and density-based (DHHM) hierarchical hashing methods on CORDIS dataset and 70 topics.

\begin{tabular}{c|rr||rr||rr}
\multicolumn{9}{c}{ CORDIS-150 (data-ratio) } \\
\hline \multirow{2}{*}{ LEVEL } & \multicolumn{2}{|c}{ THHM } & \multicolumn{2}{c}{ CHHM } & \multicolumn{2}{c}{ DHHM } \\
& mean & median & mean & median & mean & median \\
\hline \multirow{2}{*}{2} & 99.9 & 99.9 & 40.9 & 41.2 & $\mathbf{3 . 1}$ & 2.9 \\
3 & 99.9 & 99.9 & 75.3 & 76.7 & $\mathbf{6 . 2}$ & 6.1 \\
4 & 99.9 & 99.9 & 90.0 & 92.1 & $\mathbf{1 2 . 1}$ & 11.8 \\
5 & 99.9 & 99.9 & 96.4 & 96.9 & $\mathbf{2 1 . 6}$ & 20.6 \\
6 & 99.9 & 99.9 & 98.1 & 98.9 & $\mathbf{3 6 . 5}$ & 33.9 \\
\hline
\end{tabular}

Table 6.10: Data size ratio used (mean and median) of threshold-based (THHM), centroid-based (CHHM) and density-based (DHHM) hierarchical hashing methods on CORDIS dataset and 150 topics.

We consider the result obtained as significant, based on the fact that mean and median values are fairly close. The centroid-based method (chhm) and the density-based method (dhhm) show a similar behaviour to the one offered by the use of brute force by means of JS divergence.

In terms of efficiency, we consider the times to compare pairs of topic distributions constant, and we focus on the number of comparisons needed. Thus, algorithms with larger candidate spaces will be less efficient than others when the accuracy in both is the same. Tables 6.76 .12 show the percentage of the corpus used by each of the algorithms to discover related documents. Tables 6.1-6.6 show the accuracy of each algorithm for each of these scenarios. Density-based algorithm (dhhm) shows better balance between accuracy and volume of information (efficiency). It uses smaller samples (i.e lower ratio size) than others in all tests and even when it only uses a subset that is a $6.2 \%$ (Table 6.10 of the entire corpus, it obtains an accuracy of 0.808 (Table 6.4).

The precision achieved by the algorithm based on density (dhhm), which is much 


\section{PATENTS-250 (data-ratio)}

\begin{tabular}{c|rr||rr||rr}
\hline \multirow{2}{*}{ LEVEL } & \multicolumn{2}{|c}{ THHM } & \multicolumn{2}{c}{ CHHM } & \multicolumn{2}{c}{ DHHM } \\
& mean & median & mean & median & mean & median \\
\hline \multirow{2}{*}{2} & 99.9 & 99.9 & 43.2 & 32.7 & $\mathbf{3 5 . 1}$ & 23.0 \\
3 & 99.9 & 100.0 & 82.4 & 100.0 & $\mathbf{7 8 . 2}$ & 100.0 \\
4 & 99.9 & 100.0 & 96.5 & 100.0 & $\mathbf{9 5 . 1}$ & 100.0 \\
5 & 99.9 & 99.9 & 99.2 & 100.0 & $\mathbf{9 8 . 9}$ & 100.0 \\
6 & 100.0 & 100.0 & 99.8 & 100.0 & $\mathbf{9 9 . 7}$ & 100.0 \\
\hline
\end{tabular}

Table 6.11: Data size ratio used (mean and median) of threshold-based (THHM), centroid-based (CHHM) and density-based (DHHM) hierarchical hashing methods on Patents dataset and 250 topics.

PATENTS-750 (data-ratio)

\begin{tabular}{c|rr||rr||rr}
\hline \multirow{2}{*}{ LEVEL } & \multicolumn{2}{|c}{ THHM } & \multicolumn{2}{c}{ CHHM } & \multicolumn{2}{c}{ DHHM } \\
& mean & median & mean & median & mean & median \\
\hline \multirow{2}{*}{2} & 99.9 & 100.0 & 35.2 & 23.6 & $\mathbf{3 1 . 8}$ & 19.9 \\
3 & 99.9 & 99.9 & 81.4 & 99.8 & $\mathbf{7 9 . 6}$ & 98.8 \\
4 & 99.9 & 99.9 & 96.5 & 99.9 & $\mathbf{9 5 . 5}$ & 99.5 \\
5 & 97.7 & 96.6 & 99.0 & 99.9 & $\mathbf{9 8 . 6}$ & 99.7 \\
6 & 99.1 & 98.6 & 99.7 & 99.9 & $\mathbf{9 9 . 5}$ & 99.8 \\
\hline
\end{tabular}

Table 6.12: Data size ratio used (mean and median) of threshold-based (THHM), centroid-based (CHHM) and density-based (DHHM) hierarchical hashing methods on Patents dataset and 750 topics.

more restrictive than the others, suggests that few topics are required to represent a document in order to obtain related ones. In addition, the number of topics does not seem to influence the performance of the algorithms, since their precision values are similar among the datasets of the same corpus. This shows that hashing methods based on hierarchical set of topics are robust to models with different dimensions.

The behavior of the algorithms have also been analyzed when the number of topics in the model varies. Models with 100, 200, 300, 400, 500, 600, 700, 800, 900 and 1000 topics were created from the CORDIS corpus. For each model, the p@5 of the hashing methods is calculated taking into account the hierarchy levels: 2, 3, 4, 5 and 6 . Figures 6.7 to 6.9 show the results obtained for each algorithm. It can be seen how the performance, i.e precision, of each of the algorithms is not influenced by the dimensions of the model. 


\section{Precision vs Dimension}

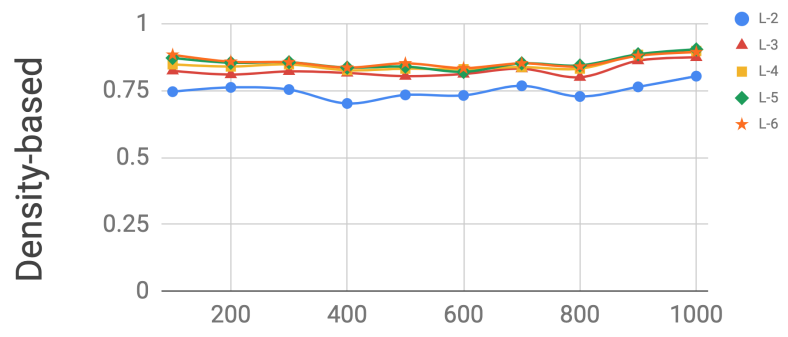

Number of Topics

Figure 6.7: Precision at 5 (mean) of threshold-based hashing method when number of topics varies in CORDIS dataset.

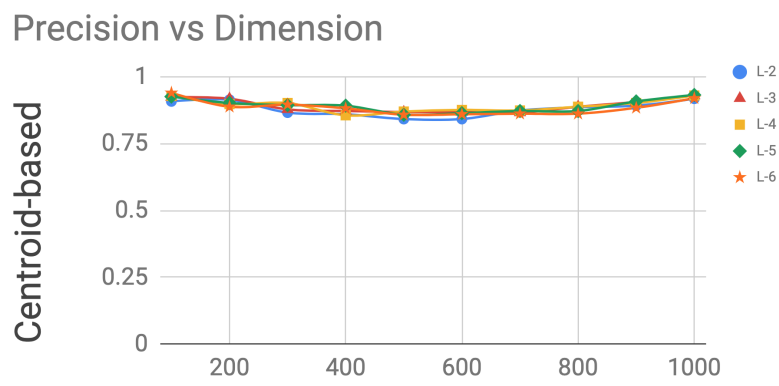

Number of Topics

Figure 6.8: Precision at 5 (mean) of centroid-based hashing method when number of topics varies in CORDIS dataset.

\subsubsection{Exploration}

In a certain domain, we may want to retrieve related documents to one given. For example, searching for articles in the Biomedical domain that are related to an article about Semantic Web. In terms of topics this kind of search requires to narrow down the initial search space to a subset with only documents that contain the topics that better describe the queried domain.

Existing hashing techniques based on a binary-code Hamming space do not allow to customize the search query beyond the reference document itself. However, the algorithms proposed in this chapter allow adding new restrictions to the initial query based on the reference document, since they use a hierarchy of set of topics as hash codes. 


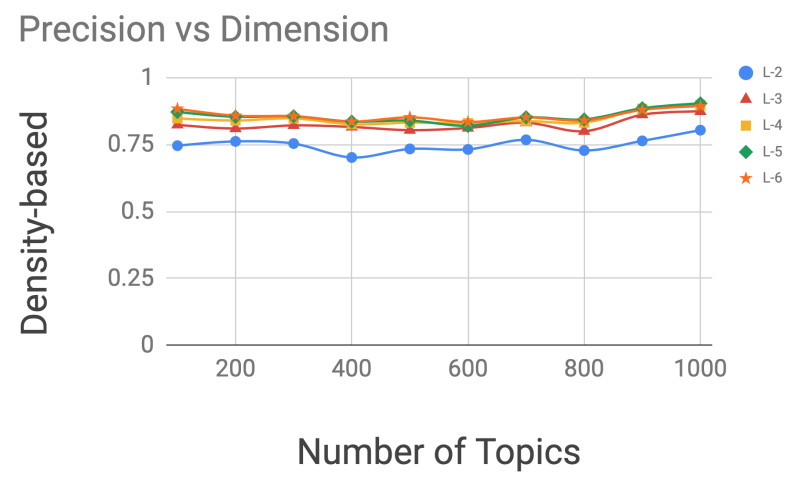

Figure 6.9: Precision at 5 (mean) of density-based hashing method when number of topics varies in CORDIS dataset.

Through the following example we describe the workflow to enable such retrieval operations. For simplicity we consider hash expressions with only two hierarchy levels. The reference document $d_{1}$ has the following hash expression: $h=\left\{h_{0}, h_{1}\right\}=$ $\{(t 10),(t 18)\}$.

The first query, $Q 1$, searches for documents related to the reference document $d_{1}$ among all documents in the corpus. One of the ways to formalise this query looks like this: $Q 1=h_{0}: t 10^{\wedge} 100$ or $h_{0}: t 18^{\wedge} 50$ or $h_{1}: t 10^{\wedge} 50$ or $h_{1}: t 18^{\wedge} 100$. It sets a maximum boost (100) when the same restrictions as the reference document ( $t 10$ in $h_{0}$ and $t 18$ in $\left.h_{1}\right)$ are fulfilled, and a lower boost (50) for the others ( $t 18$ in $h_{0}$ and $t 10$ in $\left.h_{1}\right)$. In the specific case of applying this query to the CORDIS dataset, we observed that most of the retrieved documents included topic t18 (fig 6.10).

But if we were only interested in related documents to $d_{1}$ that have topic $t 10$, we could restrict the previous query $Q 1$ to express this condition in the following way: $Q 2=\left(h_{0}: t 10^{\wedge} 100\right.$ or $\left.h_{1}: t 10^{\wedge} 50\right)$ and $\left(h_{1}: t 10^{\wedge} 50\right.$ or $\left.h_{1}: t 18^{\wedge} 100\right)$. The result obtained by $Q 2$ (fig 6.10) shows that the condition has been considered since there is a balance between topics $t 10$ and $t 18$ among the documents related to $d_{1}$.

\subsection{Summary}

The usefulness of topics created by probabilistic models when exploring document collections on large-scale has been widely studied in the literature. Each document in the corpus is described by probability distributions that measure the presence of those 


\begin{tabular}{|c||c|c||c|}
\hline \multicolumn{4}{|c|}{ OPEN-RESEARCH-100 } \\
\hline hash & q1 & q2 & ratio \\
\hline thhm & 499,755 & 160,660 & 67.8 \\
chhm & 356,111 & 1,976 & 99.44 \\
dhhm & 49,068 & 766 & 98.43 \\
\hline
\end{tabular}

Table 6.13: Number of documents related to a given one (q1) and also in a specific domain (q2) for threshold-based (thhm), centroid-based (chhm) and density-based (dhhm) hierarchical hashing methods.

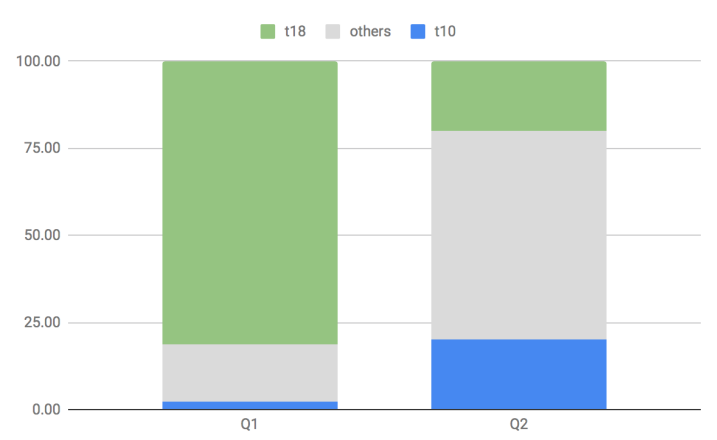

Figure 6.10: Most relevant topics in related documents from using a document as query (Q1) and setting topic t10 as mandatory (Q2).

topics in their content. These vectors can also be used to measure the similarity between documents by using metrics such as Jensen-Shannon divergence (see Section 2.2). But with large amounts of items in the collection, discovering the entire set of nearest neighbors to a given document would be infeasible. Due to the low storage cost and fast retrieval speed, hashing is one of the popular solutions for approximate nearest neighbors. However, existing hashing methods for probability distributions only focus on the efficiency of searches from a given document, without handling complex queries or offering hints about why one document is considered more or less related to another.

In this chapter we have introduced a new data structure to represent hash codes, based on topic hierarchies created from the topic distributions. This approach has proven to obtain high-precision results and can accommodate additional query restrictions based on the semantics offered by topics. In doing so, we have showed a new way to annotate documents by topic inferences made from topic models. This addresses the technical objective of this thesis T03 (integrate the annotation method based on topic hierarchies into the topic model service). 
This way of encoding documents can also help to understand why two documents are related, based on the intersection of topics at hierarchies of relevance. We have proposed a method to compare and organize huge document collections based on similar topicbased annotations, thus addressing the research objective R05 (define nearest-neighbor techniques to organize documents in regions with similar topic hierarchies).

In addition, we have implemented the technique to compare documents in our librAIry framework, achieving the T04 technical objective (create a system capable of finding related documents automatically). 


\section{Chapter 7}

\section{Cross-lingual Document Similarity}

As stated in Chapter 3 , the last of our hypotheses aims to determine whether documents in different languages can be related without having to translate them, by using language-agnostic concepts derived from their main topics (H1.4). In particular, our goal is to find abstractions that capture the content of documents, independently from the language used, in order to draw relations between them.

A way to achieve this objective is by creating multilingual topics from comparable or parallel corpora, and relating documents from their topic distributions (See Section 2.4.4 (Boyd-Graber and Blei, 2009; Boyd-Graber and Resnik, 2010, Vulić et al., 2015). A parallel corpora contains sentence-aligned documents (e.g. Europar ${ }^{78}$ corpora) (Steinberger et al., 2014), and a comparable corpora contains theme-aligned documents (e.g. Wikipedia 79 articles) (Ni et al., 2009, 2011). Other types of abstractions may be obtained using multilingual dictionaries to translate documents in a common language from which they can be related Gutierrez et al., 2016; Liu et al., 2015a; $\mathrm{Ma}$ and Nasukawa, 2017). Machine translation techniques could alleviate the requirement of multilingual training corpora by creating language-independent word representations based on distributed representations of language (Dabre et al. 2020). Unlike classical statistical approaches (Nakov and Ng, 2009), neural machine translation framework can handle translation between more than one language pair (Chen et al., 2018, Neubig

${ }^{78}$ https://ec.europa.eu/jrc/en/language-technologies/dcep

${ }^{75}$ https://www.wikipedia.org/ 
and $\mathrm{Hu}, 2018$ ). These systems tend to generalize better due to exposure to diverse languages, leading to improved translation quality compared to bilingual systems. This particular phenomenon is known as translation knowledge transfer (Pan and Yang, 2010) (i.e. "knowledge transfer" or "transfer learning").

However, the use of machine translation techniques to create topic spaces across languages reduces the ability in describing independently topics for each language through their most representative words. Similar topics in different languages do not necessarily are described by the translation of their most representative words. For example, the topic 'economic development' created from EU legal regulations using English texts is described with the following top5 word 80 , develop, strategy, growth, regional, international, reform; while when using Spanish texts its most representative words are 81 . acción, financiación, alimentario, apoyo, compromiso. As can be seen, the most relevant words for each topic are not direct translations, but they are related around the same theme. More details about these topic models are provided further in section 7.1.3.

Modern language models also make it possible to relate terms across languages based on word sequences (Kaliyar, 2020). They assume that the probability of a word appearing in a text depends only on the previous words, taking into account that the number of words considered is variable. And for the same reason previously mentioned, "knowledge transfer", that probability can be learned in one language and inferred for others. However, topic models assume bags of words and, therefore, the sequence of words is unknown. Multilingual topics need to be related in order to be described independently by each language without compromising the ability to create unique representation spaces across languages. Approaches based on aligned corpora require prior knowledge at document-level (by parallel or comparable corpora) or at word-level (by dictionaries) to create topic models that represent documents in a common, language-independent space. In this way, the pre-established language relations condition the creation of the topics (supervised method), instead of being inferred from the topics themselves as a posteriori knowledge (non-supervised method). We propose a completely unsupervised way of building cross-lingual topic models based on sets of cognitive synonyms (synsets) (Miller, 1995) to discover relations between language-specific topics once the models (for each language) have been created. It does not require parallel or comparable data

\footnotetext{
${ }^{80}$ http://librairy.linkeddata.es/jrc-en-model/topics/330/words

81 http://librairy.linkeddata.es/jrc-es-model/topics/330/words
} 
for training (Badenes-Olmedo et al., 2019a b). The cross-lingual topic models can be used for large-scale multilingual document classification and information retrieval tasks.

In Section 7.1. we propose conceptual abstractions for topic models. Topics are no longer described by words, but by language agnostic concepts. Cross-lingual topic models were created following this approach to describe documents from the most relevant concepts of their topics. The ability of these models to classify multilingual documents and to perform cross-lingual information retrieval were evaluated.

\subsection{Synset-based Representational Space}

In our approach, we propose annotating each topic with a list of synsets (Bond and Foster, 2013) retrieved from WordNet ${ }^{82}$ based on its top $n$ words (Fig 7.1). Word by word are queried in WordNet to retrieve its synsets. The final set of synsets for a topic is the union of the synsets from the individual top-words of a topics. Based on empirical evidence from different executions of the algorithm, $n=5$ is the configuration that offered the best performance in our tests, although we will avoid this parameter in the future by using some heuristic to choose the most representative terms for each topic. Let's look at an example to clarify how it works. Given the topics of Table 7.1. the EN-Topic ("communications systems") is annotated with the following synset list: radio.a.01, radio.v.01, radio.n.03, radio.n.01, radio_receiver.n.01, equipment.n.01, network.n.02, network.n.04, network.v.01, network.n.05, network.n.01, net.n.06, communication.n.02, communication.n.03, communication.n.01, regulative.s.01. The list of synsets for the ES-Topic ("sistema de comunicación") is: kit.n.02,team.n.01, equipment.n.01, net.n.02, net.n.05, network.n.05, web.n.06, network.n.01, web.n.02, communication.n.02, communication.n.01, announcement.n.02, spectrum.n.02, spectrum.n.01, creep.n.01, ghost.n.01, apparition.n.02, electromagnetic.a.01. And the list for FRTopic ("systeme de communication") is: access.n.02, approach.n.07, approach.n.02, access.n.06, access.n.03, access.n.05, assault.n.03, bout.n.02, approach.n.01, entree.n.02, entry.n.01, entrance.n.01, entry.n.03, admission.n.01, submission.n.01, introduction.n.01. The librAIry NLP service 83 was used to identify the list of synsets from a topic description based on top words. It is based on the Open Multilingual WordNet ${ }^{84}$ Bond

\begin{tabular}{|l|l|}
\hline${ }^{82} \mathrm{https} / / /$ wordnet.princeton.edu/ \\
\hline \hline${ }^{83} \mathrm{http}: / /$ librairy.linkeddata.es/nlp \\
\hline \hline${ }^{\circ 4} \mathrm{~h}$ http://compling.hss.ntu.edu.sg/omw/
\end{tabular}


and Paik, 2012).

\subsubsection{Document representation}

Documents (i.e seen as data points in the generated topic-based space) are transformed from the original feature space based on mono-lingual topic distributions into a hierarchical-code space, so that similar data points share relevant cross-lingual concepts. Since topic models create latent themes from word co-occurrence statistics in a corpus, a cross-lingual concept specifies the knowledge about the word-word relations it contains for each language. This abstraction can be extended to cover the knowledge derived from sets of topics. The topics are obtained via LDA and hierarchically divided into groups with different degrees of semantic specificity in a document (See Chapter 6). Documents represented as a weighted mixture of latent topics (per-document topic distributions) are then annotated in these feature spaces with the relation between topics inside each hierarchy level. Regardless of their language, they are then described by cross-lingual concepts (based on WordNet-synset annotations) and hash codes are calculated to summarize their content. The hash expression sets a 3-level hierarchy of cross-lingual concepts. Topics with similar presence in a document (i.e. relevance) are grouped together in the same hierarchical level (Fig 7.1) similarly to what was presented in section 6.2. Each level of the hierarchy indicates the importance of the topic according to its distribution. Level 0 describes the topics with the highest score. Level 1 describes the topics with highest score once the first ones have been eliminated, and so on. Documents are described by vectors containing set of topics (i.e. set of synsets), where each dimension means a topic relevance. Given a document $d$ with a topic distribution $q=[t 0=0.28, t 1=0.05, t 2=0.44, t 3=0.23]$, the hash expression may be $H_{d}=(t s 2),(t s 0, t s 3),(t s 1)$. It means that topic $t 2$ described by the synset $t s 2$ is the most relevant (i.e 0.44 score), then topics $t 0$ and $t 3$ described by synsets $t s 0$ and $t s 3$ (i.e 0.28 and 0.23 scores) and, finally, topic $t 1$ described by synset $t s 1$ (i.e 0.05 ).

\subsubsection{Similarity metric}

In this workspace based on hierarchical representations of topics we use the distance metric proposed in Section 6.1.2, based on the Jaccard coefficient. This metric is mainly used for set-type data (Ji et al., 2013, Li and König, 2010; Li et al., 2012, Zhao et al. 2013) and computes the similarity of sets by looking at the relative size of their 


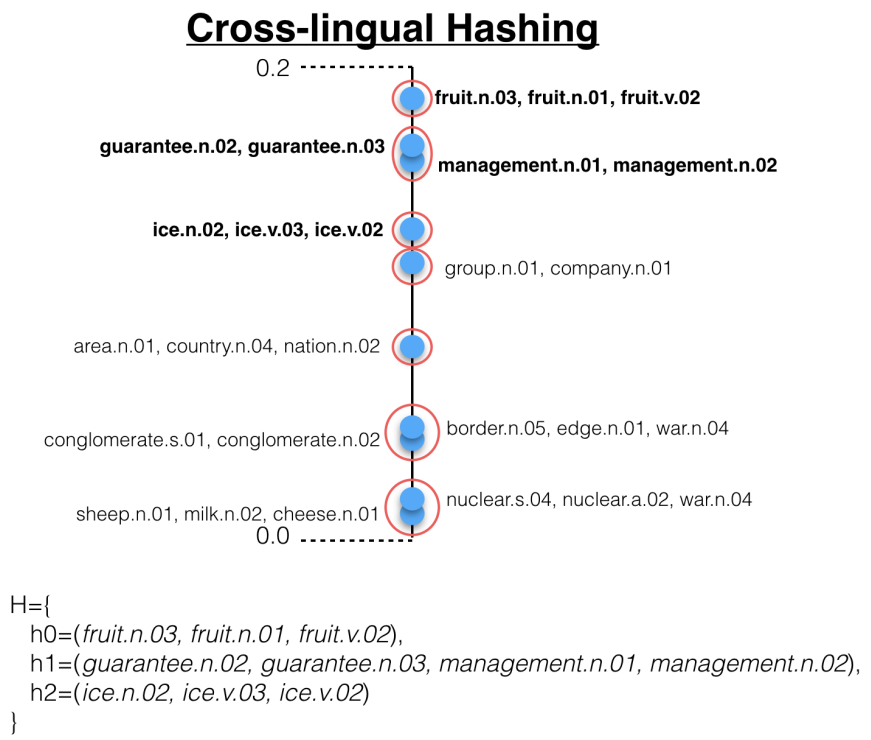

Figure 7.1: Cross-lingual hash-expression $(\mathrm{H})$ of a document based on WordNet-synset annotations created from the top words of each topic distribution. The most relevant topics are grouped according to their importance in three levels (h0, h1 and h2)

intersection (See Eq. 6.1). Thus, it allows us to measure the intersection of cross-lingual topics described by hierarchical hash-sets:

$$
d_{H}\left(H_{A}, H_{B}\right)=\sum_{l=1}^{L}\left(d_{J}\left(H_{A}\left(h_{l}\right), H_{B}\left(h_{l}\right)\right)\right)=\sum_{l=1}^{L}\left(1-\frac{H_{A}\left(h_{l}\right) \cap H_{B}\left(h_{l}\right)}{H_{A}\left(h_{l}\right) \cup H_{B}\left(h_{l}\right)}\right)
$$

where $H_{A}$ and $H_{B}$ are hash codes, $H_{A}\left(h_{l}\right)$ and $H_{B}\left(h_{l}\right)$ are the set of topics up to level $l$ for each hash code $H$, and $L$ is the maximum hierarchy level. A corner case is $L=T$, where $T$ is the number of topics in the model.

\subsubsection{Cross-lingual Models}

Our approach considers that cross-lingual models can be built from non-parallel or even non-comparable collections of multilingual documents. It first creates a probabilistic topic model for each language separately, and then annotates the topics with crosslingual labels (Fig 7.2). In the same way, the topic distribution of documents expressed through weighted vectors are first transformed into hierarchies of topics according to 

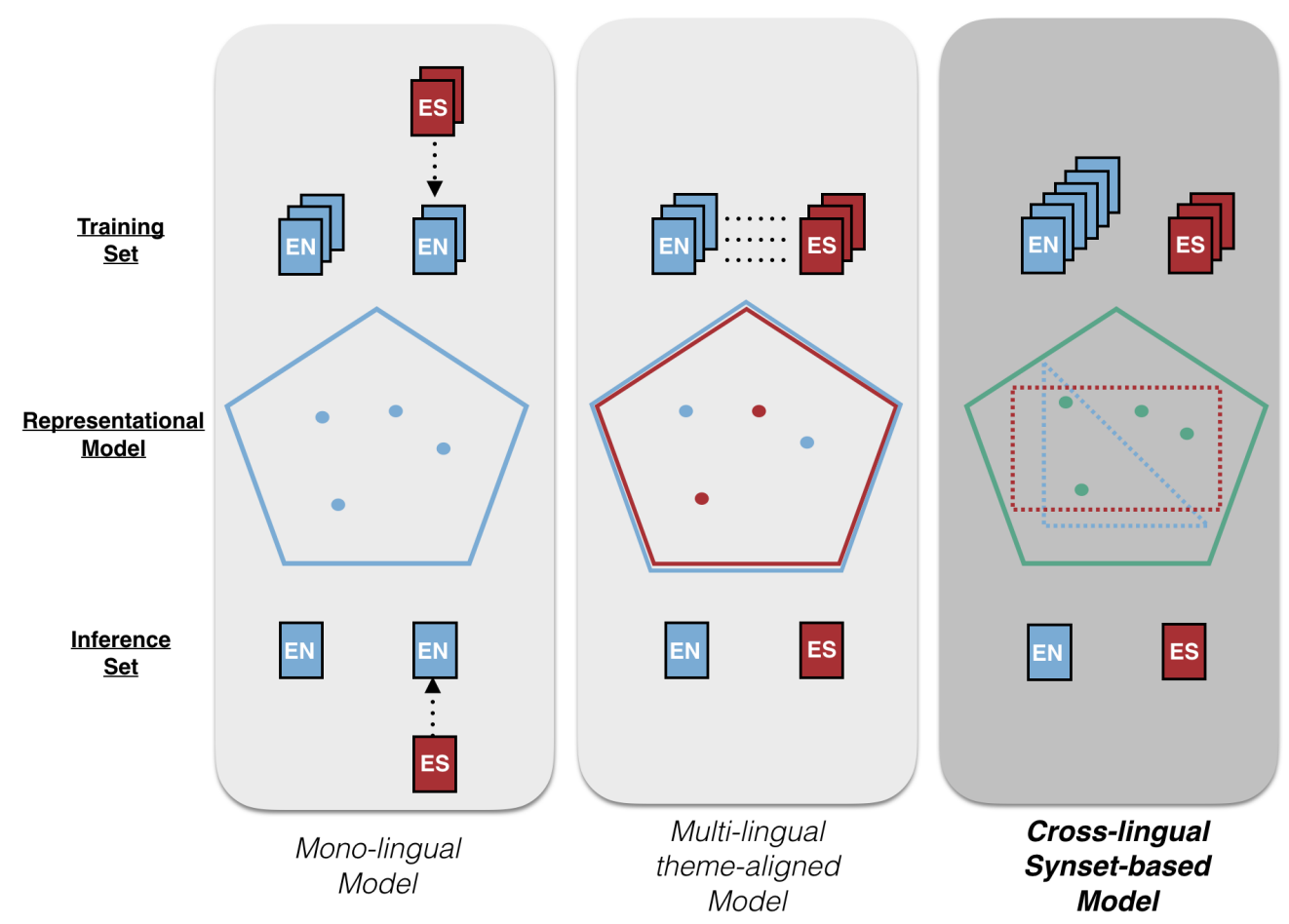

Figure 7.2: Graphical representation of the model that relies on the latent layer of crosslingual topics obtained by LDA and hash functions through hierarchies of synsets. Monolingual approaches force to translate the documents to the same language to represent them in a unique feature space. Multi-language approaches require previously aligned topics from different languages so that documents can be represented in an equivalent feature space. Cross-lingual Synset-based approach creates a new space by combining the feature spaces of each language (i.e synsets from topn topic words). Documents are then represented in this unique space.

their relevance. And then documents are described by a 3-level hierarchy of crosslingual concepts.

A pre-processing of the documents is required to clean texts and to build a suitable data set for the model. According to (Schofield et al., 2017), to obtain the benefit of a stoplist, it suffices to remove the most frequent, obvious stopwords from a corpus without developing a specific stoplist for the problem setting. We assumed terms present in more than $90 \%$ of the corpus, or in less than $0.5 \%$ of the corpus, can be considered stopwords and removed from the model. Words were normalized using lemmatized expressions of names, verbs and adjectives to create the bag-of-words, and documents with less than 100 characters were discarded since LDA has proven to have 
lower performance with these type of texts (Cheng et al., 2014).

We use the Gibbs samplers for 1000 training iterations on LDA from our librAIry framework. The Dirichlet priors $\alpha=0.1$ and $\beta=0.01$ are set following (Hu et al., 2014). Once the word distributions for each topic is available, the list of synsets related with the top5 words for each topic are identified (this number is set to offer better performance after trying several alternatives). The initial 3-level hierarchy of topics per document is replaced by a 3-level hierarchy of synsets.

\subsection{Evaluation}

In order to be able to compare the performance of our unsupervised algorithm with a semi-supervised algorithm (MuPTM-based) it is necessary to use theme-aligned corpora that map topics across languages. We used the JRC-Acquis 85 corpora (Steinberger et al. 2006). It is a collection of legislative texts written in 23 languages, although we only use English, Spanish, French, Italian and Portuguese editions for the tests. Most texts have been manually classified into subject domains according to the EUROVOC 86 thesaurus (Eurovoc, 1995), which exists in one-to-one translations into approximately twenty languages and distinguishes about 6,000 hierarchically organised descriptors (subject domains). More than 20k documents were used for each language-specific model, a total of 112,569 texts are included in the training-test package, which is publicly available ${ }^{87}$ for reuse.

The EUROVOC categories are shared among languages and will serve as support for building the topic models. They must first be moved to their base concepts and therefore disjointed categories to satisfy the topic independence assumption (Blei et al., 2003) of LDA models. The EUROVOC taxonomy has 7,193 concepts/labels from 21 domain areas such as politics, international relations, european union, law, economics, etc. There are 4,904 reciprocal hierarchical relationships (no polyhierarchy) and 6,992 reciprocal associative relationships. Using hierarchical relations, we identified the root concepts from which all other categories derive. The initial 7,193 labels were then reduced to 452 labels, which are independent (topic independence assumption from

\footnotetext{
${ }^{85} \mathrm{https}$ ///ec.europa.eu/jrc/en/language-technologies/jrc-acquis

${ }^{86}$ http://eurovoc.europa.eu/

${ }^{87}$ http://librairy.linkeddata.es/data/jrc/select?q=*:*
} 
LDA is satisfied), and can be used to train the topic models following a semi-supervised approach.

\begin{tabular}{ccccc}
\hline EN-Topic 3 & ES-Topic 3 & FR-Topic 26 & PT-Topic 10 & IT-Topic 3 \\
"communications & "sistema de & "systeme de & "meios de & "strutture di \\
systems" & comunicación" & communication" & comunicação" & comunicazione" \\
\hline radio & equipo & communications & rede & rete \\
equipment & red & reseaux & comunicação & comunicazione \\
network & comunicación & electroniques & electronico & apparecchiatura \\
communication & espectro & acces & acesso & radio \\
regulatory & electromagnético & telecommunications & utilizador & regolamentazione \\
spectrum & electrónico & service & operador & spettro \\
electronic & reglamentación & universel & regulador & elettronico \\
access & banda & reglamentaires & universal & armonizzare \\
standard & etsir & nationales & garantir & mobile \\
mobile & compatibilidad & fourniture & regulamentar & banda \\
\hline
\end{tabular}

Table 7.1: Randonly selected theme-aligned topics described by top 10 words based on EUROVOC annotations from JRC-Acquis dataset

We need to use a variant of LDA to force the correspondence between the 452 root categories identified in the EUROVOC thesaurus and the latent topics of the model. Thus, LabeledLDA (Ramage et al., 2009), a supervised version of LDA, was used to perform parameter estimation. Theme-aligned probabilistic topic models in Spanish 88 English 89 , French 90 Italian ${ }^{91}$ and Portuguese ${ }^{92}$ were created sharing the topics but not its definitions (i.e. vocabulary) (see table 7.1).

Our unsupervised approach used the same corpora created from JRC-Acquis but without using the EUROVOC categories. Topic models were created using LDA algorithm and Gibss sampling, as described in the previous section. The number of topics was set to $K=500$ (several configurations were evaluated, but this was the closest to the performance obtained with the supervised model based on categories). Probabilis-

\footnotetext{
${ }^{88} \mathrm{http}: / /$ librairy.linkeddata.es/jrc-es-model

${ }^{89} \mathrm{http}: / /$ librairy.linkeddata.es/jrc-en-model

${ }^{90}$ http://librairy.linkeddata.es/jrc-fr-model

${ }^{91}$ http://librairy.linkeddata.es/jrc-it-model

${ }^{92}$ http://librairy.linkeddata.es/jrc-pt-model
} 
tic topic models in Spanish $\sqrt{93}$ English 94 , French 95 Italian ${ }^{96}$ and Portuguese ${ }^{97}$ were created independently without previously establishing any type of alignment between their topics.

A simple way of looking at the output quality of the topic models is by simply inspecting top words associated with a particular topic learned during training. A latent topic is semantically coherent if it assigns high probability scores to words that are semantically related (Gliozzo et al., 2007; Mimno et al., 2011; Newman et al., 2010). It is much easier for humans to judge semantic coherence of cross-lingual topics and their alignment across languages when observing the actual words constituting a topic. These words provide a shallow qualitative representation of the latent topic space, and could be seen as direct and comprehensive word-based summaries of a large document collection.

Samples of cross-lingual topics are provided in Table 7.1. We may consider this visual inspection of the top words associated with each topic as an initial qualitative evaluation, suitable for human judges. Documents present similar topic distributions when projecting their content on topics according to their language as can be seen in fig 7.3 . Since the topic identifiers are not aligned, the graphs appear displaced.

A way to evaluate our cross-lingual document similarity algorithm is to test how well it performs in practice for different real-life tasks: document classification and information retrieval. Evaluation is done using the B-Cubed metrics (Bagga and Baldwin, 1998) to estimate the fit between two clusters, the one obtained from a supervised category-based topic alignment algorithm and the one obtained from our unsupervised synset-based topic alignment algorithm.

Let $C L_{i}$ be the cluster that document $t_{i}$ gets clustered in, and $G_{i}$ its correct cluster from the ground truth. The B-Cubed metric then calculates precision $=\frac{\left|C L_{i} \cap G_{i}\right|}{\left|C L_{i}\right|}$ and recall $=\frac{\left|C L_{i} \cap G_{i}\right|}{\left|G_{i}\right|}$. The total precision and recall of the clustering are taken as the average of the precision and recall scores over all documents. Results are also presented in terms of the $F_{1}$ measure to balance between precision and recall: $F_{1}=\frac{2 \cdot \text { precision } \cdot \text { recall }}{\text { precision }+ \text { recall }}$.

\footnotetext{
${ }^{93} \mathrm{http}: / /$ librairy.linkeddata.es/jrc-es-model-unsupervised

${ }^{94}$ http://librairy.linkeddata.es/jrc-en-model-unsupervised

${ }^{95} \mathrm{http}: / /$ librairy.linkeddata.es/jrc-fr-model-unsupervised

${ }^{96}$ http://librairy.linkeddata.es/jrc-it-model-unsupervised

${ }^{97}$ http://librairy.linkeddata.es/jrc-pt-model-unsupervised
} 


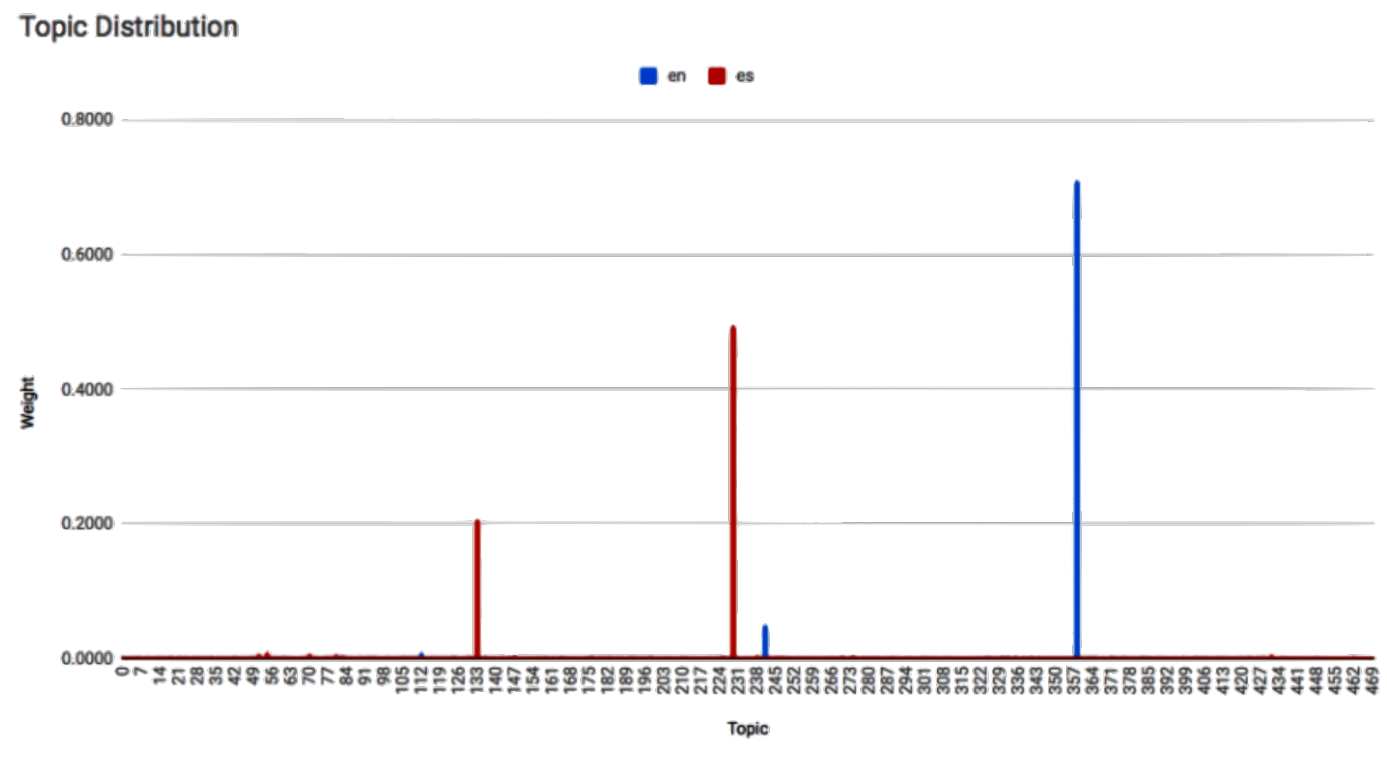

Figure 7.3: topic distributions from the same document in English $\left(h_{E N}=\right.$ $\{(t 3062),(t 335),(t 8278)\})$ and Spanish $\left(h_{E S}=\{(t 335),(t 4060),(t 5769)\}\right)$.

The aim is to measure the performance of the algorithm taking into account documents with manual category assignments.

\subsubsection{Cross-lingual Document Classification}

A random group of $1 \mathrm{k}$ documents from the JRC-Acquis corpora, which have not been used to train the models, is considered for evaluation as they are manually tagged with EUROVOC categories. For each document, the cluster to which it belongs is identified from its categories. This cluster is then compared (B-Cubed metrics) with the one obtained from the labels generated from its most representative topics (cat) and with the one obtained from the labels generated with the WordNet-Synsets of those topics (syn). Algorithm performance is evaluated in monolingual, bilingual, and multilingual document collections (tables 7.2 and 7.3 ) .

The results show a higher performance of the semi-supervised algorithm (categoriesbased topic alignment) in terms of precision, and of the unsupervised algorithm (synsetbased topic alignment) in terms of coverage. The cause lies in the set of synonyms generated by WordNet, being able to share the same synset for two different topics. From a more general point of view (fMeasure), the benefit obtained by the increase in 


\begin{tabular}{|c|c|c|c|c|c|c|c|c|c|c|c|}
\hline \multicolumn{12}{|c|}{ JRC-Acquis Corpora } \\
\hline & & \multicolumn{2}{|c|}{ en } & \multicolumn{2}{|c|}{ es } & \multicolumn{2}{|c|}{ fr } & \multicolumn{2}{|c|}{ pt } & \multicolumn{2}{|c|}{ it } \\
\hline & & cat & syn & cat & syn & cat & syn & cat & syn & cat & syn \\
\hline \multirow{4}{*}{ prec } & $\min$ & 0.01 & 0.01 & 0.01 & 0.01 & 0.01 & 0.01 & 0.01 & 0.01 & 0.01 & 0.01 \\
\hline & $\max$ & 1.00 & 0.95 & 1.00 & 0.87 & 1.00 & 0.87 & 1.00 & 0.83 & 1.00 & 0.85 \\
\hline & mean & 0.58 & 0.48 & 0.55 & 0.48 & 0.55 & 0.41 & 0.53 & 0.42 & 0.54 & 0.45 \\
\hline & $d e v$ & 0.27 & 0.23 & 0.27 & 0.22 & 0.26 & 0.20 & 0.24 & 0.21 & 0.25 & 0.22 \\
\hline \multirow{4}{*}{ rec } & $\min$ & 0.01 & 0.03 & 0.01 & 0.04 & 0.01 & 0.05 & 0.01 & 0.04 & 0.01 & 0.05 \\
\hline & $\max$ & 0.96 & 1.00 & 0.93 & 1.00 & 0.95 & 1.00 & 0.92 & 1.00 & 0.94 & 1.00 \\
\hline & mean & 0.39 & 0.52 & 0.36 & 0.49 & 0.42 & 0.51 & 0.40 & 0.47 & 0.39 & 0.48 \\
\hline & dev & 0.24 & 0.20 & 0.23 & 0.20 & 0.23 & 0.23 & 0.23 & 0.21 & 0.23 & 0.20 \\
\hline \multirow{4}{*}{ f1 } & $\min$ & 0.02 & 0.03 & 0.01 & 0.02 & 0.02 & 0.03 & 0.02 & 0.02 & 0.01 & 0.02 \\
\hline & $\max$ & 0.70 & 0.75 & 0.70 & 0.71 & 0.70 & 0.73 & 0.70 & 0.71 & 0.70 & 0.72 \\
\hline & mean & 0.35 & 0.42 & 0.32 & 0.41 & 0.37 & 0.39 & 0.35 & 0.38 & 0.31 & 0.40 \\
\hline & dev & 0.16 & 0.15 & 0.15 & 0.15 & 0.17 & 0.17 & 0.16 & 0.16 & 0.16 & 0.15 \\
\hline
\end{tabular}

Table 7.2: Document classification performance (precision-'prec', recall-'rec' and fMeasure-'f1') of the categories-based (cat) and synset-based (syn) topic alignment algorithms in monolingual document collections

coverage (recall) is greater than by the loss of accuracy (precision).

\subsubsection{Cross-lingual Information Retrieval}

Given a set of documents and a text, the task is to rank the documents according to their relevance to the query text regardless of the language used. The JRC-Acquis corpus is used because by having texts tagged with EUROVOC categories we can build a ground-truth set grouping the documents that share the same codes as those used in the query document. A collection of $1 \mathrm{k}$ randomly selected documents (monolingual, bilingual and multi-lingual) are annotated by the category-based and synset-based topic alignment algorithms. Then, we randomly take articles to search in D for documents that share the same categories than the query document (i.e the ground-truth set). Next, the query text is used to search in $\mathrm{D}$ for similar documents using categorybased annotations and synset-based annotations. We evaluate the performance of the algorithms in terms of precision@3, precision@5 and precision@10 (tables 7.4 and 7.5 )

Although the precision values are lower than those obtained by semi-supervised 


\begin{tabular}{|c|c|c|c|c|c|c|c|c|c|}
\hline \multicolumn{10}{|c|}{ JRC-Acquis Corpora } \\
\hline & & \multicolumn{2}{|c|}{ en-es } & \multicolumn{2}{|c|}{ en-es-fr } & \multicolumn{2}{|c|}{ en-es-fr-pt } & \multicolumn{2}{|c|}{ en-es-fr-pt-it } \\
\hline & & cat & syn & cat & syn & cat & syn & cat & syn \\
\hline \multirow{4}{*}{ prec } & $\min$ & 0.01 & 0.01 & 0.01 & 0.01 & 0.01 & 0.01 & 0.01 & 0.01 \\
\hline & $\max$ & 1.00 & 0.97 & 1.00 & 0.98 & 1.00 & 0.97 & 1.00 & 0.98 \\
\hline & mean & 0.62 & 0.55 & 0.59 & 0.52 & 0.56 & 0.50 & 0.57 & 0.52 \\
\hline & dev & 0.26 & 0.23 & 0.26 & 0.25 & 0.26 & 0.26 & 0.26 & 0.26 \\
\hline \multirow{4}{*}{ rec } & $\min$ & 0.01 & 0.09 & 0.01 & 0.07 & 0.01 & 0.06 & 0.01 & 0.04 \\
\hline & $\max$ & 1.00 & 1.00 & 0.86 & 0.93 & 0.83 & 0.91 & 0.80 & 0.89 \\
\hline & mean & 0.33 & 0.57 & 0.25 & 0.39 & 0.21 & 0.36 & 0.23 & 0.37 \\
\hline & dev & 0.16 & 0.23 & 0.13 & 0.15 & 0.12 & 0.13 & 0.12 & 0.15 \\
\hline \multirow{4}{*}{ f1 } & $\min$ & 0.02 & 0.02 & 0.02 & 0.05 & 0.02 & 0.06 & 0.02 & 0.08 \\
\hline & $\max$ & 0.75 & 0.81 & 0.62 & 0.66 & 0.61 & 0.64 & 0.59 & 0.62 \\
\hline & mean & 0.36 & 0.49 & 0.30 & 0.38 & 0.29 & 0.35 & 0.30 & 0.36 \\
\hline & dev & 0.16 & 0.18 & 0.11 & 0.12 & 0.11 & 0.11 & 0.11 & 0.12 \\
\hline
\end{tabular}

Table 7.3: Document classification performance (precision-'prec', recall-'rec' and fMeasure-'f1') of the categories-based (cat) and synset-based (syn) topic alignment algorithms in multi-lingual document collections

approximation, they are sufficiently promising (around 0.75) to think that introducing improvements in the lemmatization process would increase the quality of the WordNetsynset annotations derived from the most representative words of each topic (precision values close to 0.8 in the English corpus).

\subsubsection{Text Length on Cross-lingual Representations}

The ability of topics to materialize the underlying knowledge of the documents depend on the texts used to train models. We have studied the impact that the length of texts has, since they determine the space where words can co-occur, to semantically relate multilingual documents described in a probabilistic topic space and, therefore, to capture the knowledge derived from their relationships (Lozano et al., 2020).

The aim is to know how text length influences the cross-lingual topics created to semantically relate multilingual documents through state-of-the-art similarity metrics (See Chapter 2). We have designed several document retrieval tasks based on the models described in Section 7.1.3, to compare the clusters of documents created from 


\begin{tabular}{|c|c|c|c|c|c|c|c|c|c|c|c|}
\hline \multicolumn{12}{|c|}{ JRC-Acquis Corpora } \\
\hline & & \multicolumn{2}{|c|}{ en } & \multicolumn{2}{|c|}{ es } & \multicolumn{2}{|c|}{ fr } & \multicolumn{2}{|c|}{ pt } & \multicolumn{2}{|c|}{ it } \\
\hline & & cat & syn & cat & syn & cat & syn & cat & syn & cat & syn \\
\hline \multirow{2}{*}{ p@3 } & mean & 0.84 & 0.83 & 0.81 & 0.78 & 0.83 & 0.74 & 0.79 & 0.78 & 0.80 & 0.75 \\
\hline & dev & 0.26 & 0.26 & 0.27 & 0.29 & 0.26 & 0.32 & 0.27 & 0.29 & 0.27 & 0.29 \\
\hline \multirow{2}{*}{$\mathrm{p} @ 5$} & mean & 0.82 & 0.80 & 0.79 & 0.75 & 0.80 & 0.72 & 0.77 & 0.75 & 0.78 & 0.72 \\
\hline & dev & 0.25 & 0.25 & 0.25 & 0.27 & 0.25 & 0.29 & 0.25 & 0.26 & 0.26 & 0.28 \\
\hline \multirow{2}{*}{ p@10 } & mean & 0.77 & 0.76 & 0.75 & 0.73 & 0.77 & 0.68 & 0.72 & 0.71 & 0.74 & 0.68 \\
\hline & dev & 0.23 & 0.25 & 0.25 & 0.27 & 0.24 & 0.27 & 0.25 & 0.27 & 0.25 & 0.26 \\
\hline
\end{tabular}

Table 7.4: Information retrieval performance (precision@3, precision@5 and precision@10) of the categories-based (cat) and synset-based (syn) topic alignment algorithms in monolingual document collections

\begin{tabular}{cc|rr||rr||rr||rr}
\hline \multicolumn{7}{c}{ JRC-Acquis Corpora } \\
\hline & \multirow{2}{*}{} & \multicolumn{2}{c}{ en-es } & \multicolumn{2}{c}{ en-es-fr } & \multicolumn{2}{c}{ en-es-fr-pt } & \multicolumn{2}{c}{ en-es-fr-pt-it } \\
& cat & syn & cat & syn & cat & syn & cat & syn \\
\hline \multirow{2}{*}{$\mathbf{p} @ \mathbf{3}$} & mean & $\mathbf{0 . 8 4}$ & 0.79 & $\mathbf{0 . 8 5}$ & 0.75 & $\mathbf{0 . 8 1}$ & 0.69 & $\mathbf{0 . 8 2}$ & 0.71 \\
& dev & 0.25 & 0.28 & 0.24 & 0.31 & 0.23 & 0.29 & 0.25 & 0.29 \\
\hline \multirow{2}{*}{$\mathbf{p @ 5}$} & mean & $\mathbf{0 . 8 2}$ & 0.76 & $\mathbf{0 . 8 1}$ & 0.72 & $\mathbf{0 . 7 8}$ & 0.67 & $\mathbf{0 . 7 9}$ & 0.69 \\
& dev & 0.24 & 0.26 & 0.23 & 0.27 & 0.24 & 0.25 & 0.21 & 0.26 \\
\hline \multirow{2}{*}{$\mathbf{p} @ \mathbf{1 0}$} & mean & $\mathbf{0 . 7 8}$ & 0.73 & $\mathbf{0 . 7 6}$ & 0.67 & $\mathbf{0 . 7 3}$ & 0.62 & $\mathbf{0 . 7 4}$ & 0.63 \\
& dev & 0.22 & 0.24 & 0.23 & 0.26 & 0.22 & 0.24 & 0.23 & 0.24
\end{tabular}

Table 7.5: Information retrieval performance (precision@3, precision@5 and precision@10) of the categories-based (cat) and synset-based (syn) topic alignment algorithms in multi-lingual document collections

their manual annotations (i.e. EUROVOC tags) with those created automatically from their topic distributions (Fig,7.4).

The JRC-Acquis dataset used in the previous experiments (Section 7.2.1 and 7.2.2, was extended with the DGT-Acquis (Steinberger et al., 2014) collection to increase the total number of documents and the diversity of text length. It contains documents from the Official Journal of the European Union from 2004 to 2011. Given that both datasets are constructed from the same domain with no overlap for data since 2007, we decided to merge both of them into a single collection, the Acquis corpus, formed with all the JRC-Acquis dataset and the documents of DGT-Acquis from that year. 


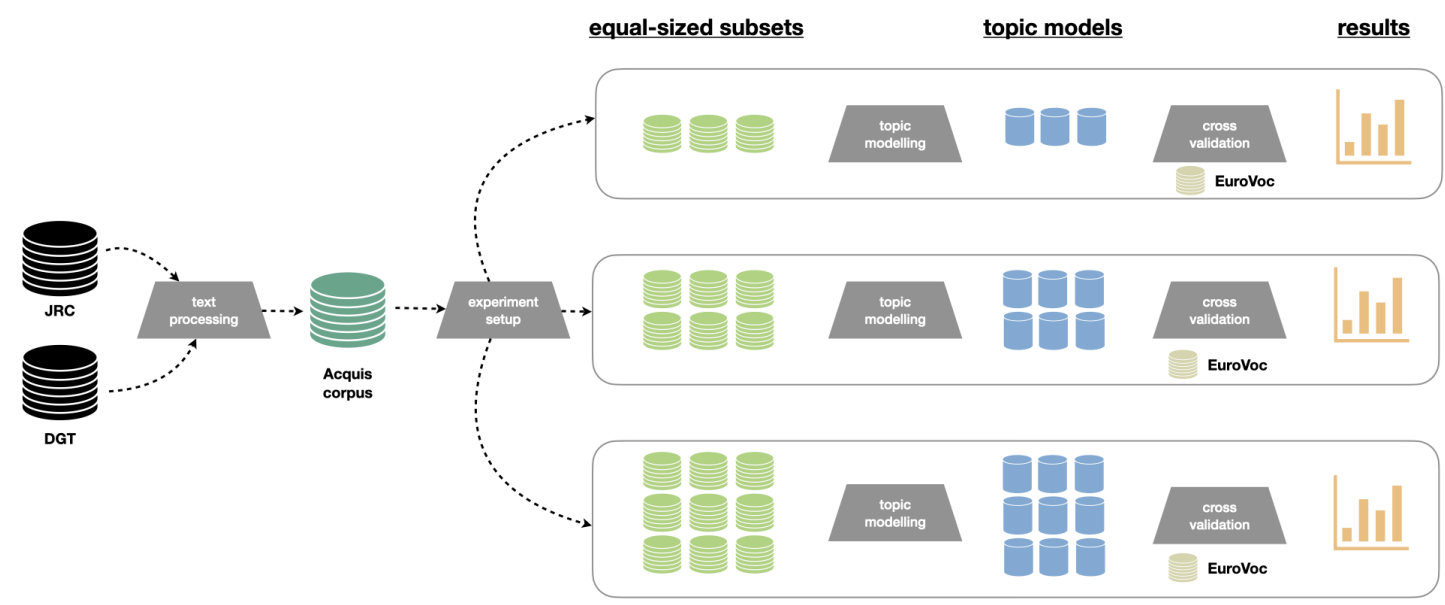

Figure 7.4: Preparation of experiments by creating topic models for each subset of the original corpus and cross-validated with EuroVoc thesaurus

English and Spanish versions of the Acquis corpus were used to validate the results across languages (see table 7.6 for a summary of the data used).

\begin{tabular}{|c|c|c|c|c|c|c|c|}
\hline & \multicolumn{3}{|c|}{ English } & \multicolumn{3}{|c|}{ Spanish } \\
\hline & & DGT & JRC & Acquis & DGT & JRC & Acquis \\
\hline Doc & ments & 51521 & 16260 & 67781 & 51585 & 16470 & 68055 \\
\hline \multirow{5}{*}{ Tokens } & Median & 135 & 197 & 152 & 129 & 204 & 150 \\
\hline & Mean & 185.8762 & 261.9931 & 204.1359 & 181.9172 & 271.2842 & 203.5449 \\
\hline & Variance & 34806.26 & 35716.91 & 36080.66 & 34624.02 & 38700.03 & 37074.97 \\
\hline & Min & 7 & 7 & 7 & 6 & 6 & 6 \\
\hline & $\operatorname{Max}$ & 1360 & 1063 & 1360 & 1411 & 1110 & 1411 \\
\hline
\end{tabular}

Table 7.6: Number of documents and tokens by dataset

Texts were pre-processed before the models were trained. We removed stopwords, including general NLP and domain-specific ones based on topic distributions. Rare terms with extremely low total document frequency were also removed. Words were lemmatized and changed to lower-case. A lower and an upper limit on the number of words (i.e. tokens) were defined to discard texts. These bounds were inferred from the interquartile range (Fig. 7.7).

We then divided the original corpora into several subsets $(3,6$ and 9$)$ of the same size with texts of similar length. The aim is to compare how similarity metrics based on topic 


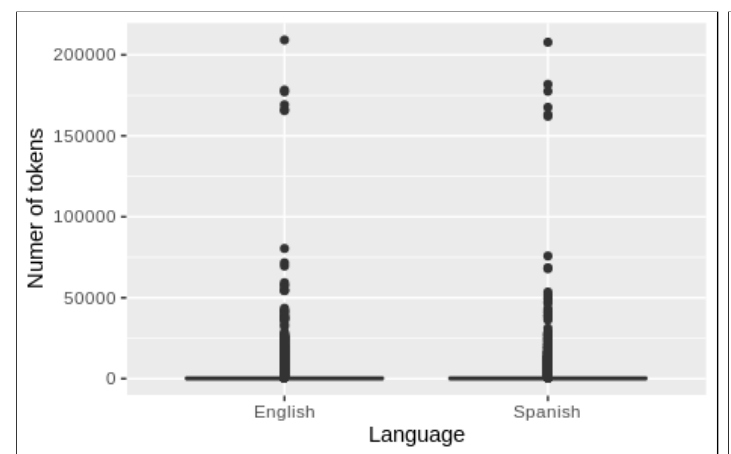

Figure 7.5: Before pre-processing

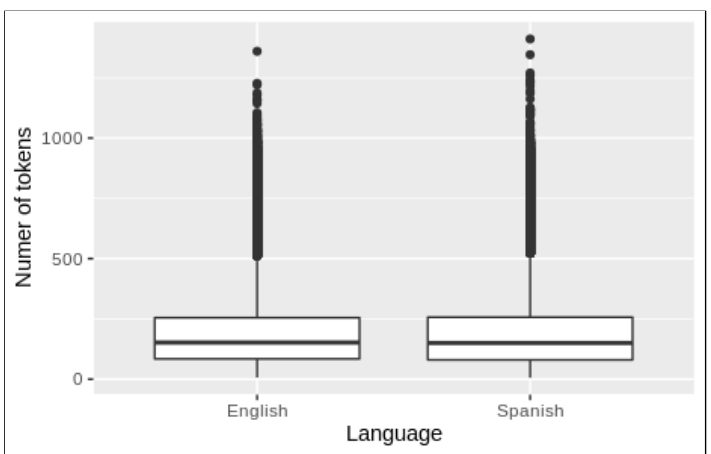

Figure 7.6: After pre-processing

Figure 7.7: Distribution of articles by number of tokens in corpora

distributions behave for each dataset in document retrieval tasks and to understand the influence of text lengths in the quality of multilingual topic models. For each data set, we reserved a sample (5\%) for testing and the rest (95\%) was used to train a topic model. The test set was projected in the topic space according to the trained model and then the similarity metrics were used to compare those documents with all documents in the corpus and obtain the most similar ones. The list with the top10 most similar documents is compared in terms of Mean Average Precision (MAP) with the one obtained when comparing them from the EuroVoc labels. This metric allows evaluating on average how good the first 10 results of a query are by taking the mean of all average precision for the first 10 results when comparing a list of retrieved documents and the ground truth.

Each document was manually annotated with one or more EuroVoc categories. Documents that share the same categories were therefore considered semantically related. For each document in the test set, its topic-based similarity to the others is calculated according to density-based metrics 2.2) such as Jensen-Shannon divergence (JSD) and Hellinger distance (HE); and our hierarchy-based metric 6.1.2 that we named Weighted Jaccard Levels (WJL) in experiments. The list of related documents from the EuroVoc categories is then compared to the list of related documents by topics expressed by density or by hierarchies. Since several topic models have been created for each dataset (with 50,100,300 and 500 topics), the precision results for each model were averaged following the mean average precision (MAP) metric. Thus, results reflect the capacity of each topic model to express the knowledge required to relate texts 
from their content without supervision. Table 7.7 shows results for each trained model and each similarity metric. A distinction is made between texts written in English and Spanish.

\begin{tabular}{c|c|ccc}
\multicolumn{5}{c}{ Acquis (MAP@10) } \\
\hline Lang & Topics & JSD & HE & WJL \\
\hline \multirow{5}{*}{ Spanish } & 50 & $\mathbf{0 . 8 0 0 6 0}$ & 0.79665 & 0.70583 \\
& 100 & $\mathbf{0 . 8 2 7 4 1}$ & 0.77930 & 0.75555 \\
& 300 & $\mathbf{0 . 8 4 2 6 1}$ & 0.58531 & 0.79036 \\
& 500 & $\mathbf{0 . 8 1 2 3 8}$ & 0.68482 & 0.79336 \\
\hline \multirow{5}{*}{ English } & 50 & $\mathbf{0 . 8 1 4 2 1}$ & 0.80150 & 0.73367 \\
& 100 & $\mathbf{0 . 8 5 5 1 0}$ & 0.74060 & 0.80315 \\
& 300 & $\mathbf{0 . 8 4 0 0 5}$ & 0.52082 & 0.83277 \\
& 500 & 0.78874 & 0.43636 & $\mathbf{0 . 8 4 5 5 5}$ \\
\hline \hline
\end{tabular}

Table 7.7: Aggregated MAP results by metric and model

The results suggest that PTM highly capture the knowledge required to relate semantically documents, since all models tested had at least one metric above 0.8 in precision. Among the metrics used to relate documents, the Jensen-Shannon divergence (JSD) offers a better performance in general terms compared to the other approaches. Although a downward trend is suggested in density-based metrics when increasing the number of topics, compared to hierarchical metrics that improve their performance when increasing the number of topics. This happens because the sum of distances of the less representative topics for JSD gets bigger as the number of topics diverge from its optimum while activated topics (i.e selected topics at one of the hierarchy levels) get more discriminative which lead to an increase of WJL. Another way to think about the number of topics is the level of detail they capture. Models with low number of topics will present general themes shared by all documents. On the contrary, with more dimensions topics are able to discriminate particular thematics only shared a subset of the document in the collection which is analogous to how EUROVOC and other theasurus works. Hierarchical metrics work best on high-dimensional topic representations because their calculations are based only on the most relevant topics. Therefore, we can conclude that automatically generated annotations from topic models offer a knowledge close to that offered by those manually assigned from the EuroVoc 
thesaurus in the Acquis legal corpus to relate texts. In the case of large and very heterogeneous collections, i.e. with a high number of different topics, it would be more appropriate to annotate documents by topic hierarchies. In view of these results, the knowledge offered by topics allows automatically discovering what is being treated in a collection of documents, and the knowledge offered by its hierarchical representation allows understanding why documents are related in a similar way as it would be done with manually assigned labels.

\begin{tabular}{|c|c|c|c|c|c|c|c|c|}
\hline & & & \multicolumn{6}{|c|}{ Acquis-3 (MAP@10) } \\
\hline & & & \multicolumn{6}{|c|}{ Training Set } \\
\hline & & & \multicolumn{2}{|c|}{1} & \multicolumn{2}{|c|}{2} & \multicolumn{2}{|c|}{3} \\
\hline & & & es & en & es & en & es & en \\
\hline \multirow{6}{*}{ 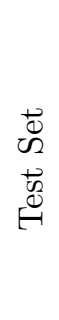 } & & $J S D$ & 0.85 & 0.83 & 0.86 & 0.85 & 0.87 & 0.87 \\
\hline & 1 & $W J L$ & 0.85 & 0.85 & 0.86 & 0.86 & 0.85 & 0.86 \\
\hline & & $J S D$ & 0.80 & 0.75 & 0.77 & 0.75 & 0.82 & 0.80 \\
\hline & 2 & $W J L$ & 0.73 & 0.77 & 0.81 & 0.83 & 0.82 & 0.83 \\
\hline & & $J S D$ & 0.72 & 0.62 & 0.68 & 0.65 & 0.69 & 0.68 \\
\hline & 3 & $W J L$ & 0.55 & 0.65 & 0.67 & 0.72 & 0.73 & 0.77 \\
\hline
\end{tabular}

Table 7.8: MAP results by dividing the corpus into three equal subsets to train and evaluate the models in English(en) and $\operatorname{Spanish}(e s)$

To better understand how the length of the texts affects the creation of probabilistic topics, we have prepared three different scenarios that divided the original corpora into subsets with similar text sizes. In the first scenario we have created three equal sets (Table 7.8), in the second scenario there were six subsets (Table 7.9p), and in the third scenario a total of nine subsets were created (Table 7.10). We have only considered the similarities calculated from JSD and WJL, as they offered the best performance for each approach.

Models created from texts (training set) with greater or equal length to the texts used in the inferences (test set) offered better performance in document retrieval tasks regardless of the language used. This is evidenced by the fact that those models performed better for almost all sets. Although for some evaluations of small documents models trained with large texts didn't yield the best performances they were not significantly different from the best models. For small documents both metrics performed similarly. 


\begin{tabular}{|c|c|c|c|c|c|c|c|c|c|c|c|c|c|}
\hline \multicolumn{14}{|c|}{ Acquis-6 (MAP@10) } \\
\hline & & \multicolumn{12}{|c|}{ Training Set } \\
\hline & & \multicolumn{2}{|c|}{1} & \multicolumn{2}{|c|}{2} & \multicolumn{2}{|c|}{3} & \multicolumn{2}{|c|}{4} & \multicolumn{2}{|c|}{5} & \multicolumn{2}{|c|}{6} \\
\hline & & es & en & es & en & es & en & es & en & es & en & es & en \\
\hline \multirow{12}{*}{$\begin{array}{l}\vec{U} \\
\tilde{D} \\
\vec{b} \\
\vec{b}\end{array}$} & jsd & 0.79 & 0.76 & 0.79 & 0.77 & 0.79 & 0.78 & 0.79 & 0.77 & 0.79 & 0.77 & 0.77 & 0.73 \\
\hline & $1 \quad w j l$ & 0.78 & 0.74 & 0.78 & 0.76 & 0.77 & 0.77 & 0.78 & 0.76 & 0.78 & 0.76 & 0.74 & 0.69 \\
\hline & , jsd & 0.82 & 0.80 & 0.81 & 0.77 & 0.80 & 0.76 & 0.81 & 0.79 & 0.85 & 0.82 & 0.84 & 0.84 \\
\hline & $2 \quad w j l$ & 0.81 & 0.82 & 0.85 & 0.86 & 0.84 & 0.86 & 0.85 & 0.85 & 0.85 & 0.85 & 0.83 & 0.84 \\
\hline & $j s d$ & 0.76 & 0.73 & 0.78 & 0.73 & 0.72 & 0.68 & 0.78 & 0.71 & 0.81 & 0.75 & 0.81 & 0.76 \\
\hline & w wjl & 0.73 & 0.70 & 0.72 & 0.78 & 0.81 & 0.79 & 0.81 & 0.80 & 0.82 & 0.80 & 0.79 & 0.80 \\
\hline & $j s d$ & 0.69 & 0.68 & 0.72 & 0.67 & 0.71 & 0.68 & 0.68 & 0.63 & 0.73 & 0.69 & 0.74 & 0.71 \\
\hline & $4 \quad w j l$ & 0.63 & 0.69 & 0.66 & 0.72 & 0.74 & 0.76 & 0.77 & 0.78 & 0.77 & 0.79 & 0.75 & 0.79 \\
\hline & $j s d$ & 0.62 & 0.57 & 0.69 & 0.61 & 0.66 & 0.62 & 0.67 & 0.59 & 0.63 & 0.59 & 0.70 & 0.65 \\
\hline & b wjl & 0.60 & 0.63 & 0.57 & 0.64 & 0.65 & 0.69 & 0.70 & 0.71 & 0.73 & 0.74 & 0.72 & 0.75 \\
\hline & $j s d$ & 0.55 & 0.52 & 0.67 & 0.56 & 0.61 & 0.56 & 0.63 & 0.56 & 0.63 & 0.56 & 0.59 & 0.55 \\
\hline & o wjl & 0.51 & 0.57 & 0.51 & 0.60 & 0.58 & 0.63 & 0.63 & 0.67 & 0.66 & 0.70 & 0.69 & 0.71 \\
\hline
\end{tabular}

Table 7.9: MAP results by dividing the corpus into six equal subsets to train and evaluate the models in English(en) and Spanish(es)

On the other hand, as the document in the test set get bigger WJL significantly outperformed JSD. As an extreme example look at the Acquis division in 9 groups. The results for the evaluation of the $9^{\text {th }}$ set (group with biggest document) with the $9^{\text {th }}$ model (trained with the biggest document set) were $13 \%$ better in the English case and $21 \%$ better, suggesting that, with enough text data, PTM models produce sparser topic proportion vectors from which WJL metric benefits.

This perception of the efficiency of hierarchy-based metrics when the topic models are large was analyzed by capturing the computational time (in seconds) that each metric used to compare the documents (Fig.7.8). For almost all number of topics, hierarchical-based metrics are much faster than probabilistic ones. However, for small number of dimensions (i.e. topics) the topic proportion vector is not sparse enough to identify any relevant topics from the uninformative ones, resulting in hierarchies containing all topics for every document. In other words, all documents share at least one topic. Increasing the number of topics alleviates this problem to the point of archiving an almost constant time for more than 35 topics. Although density-based metrics (e.g JSD and HE) increased their computational time linearly with the representation size, JSD calculation requires computing two logarithms for each dimension in the document representation, which is way more time consuming than the HE metric square-roots. 
Acquis-9 (MAP@10)

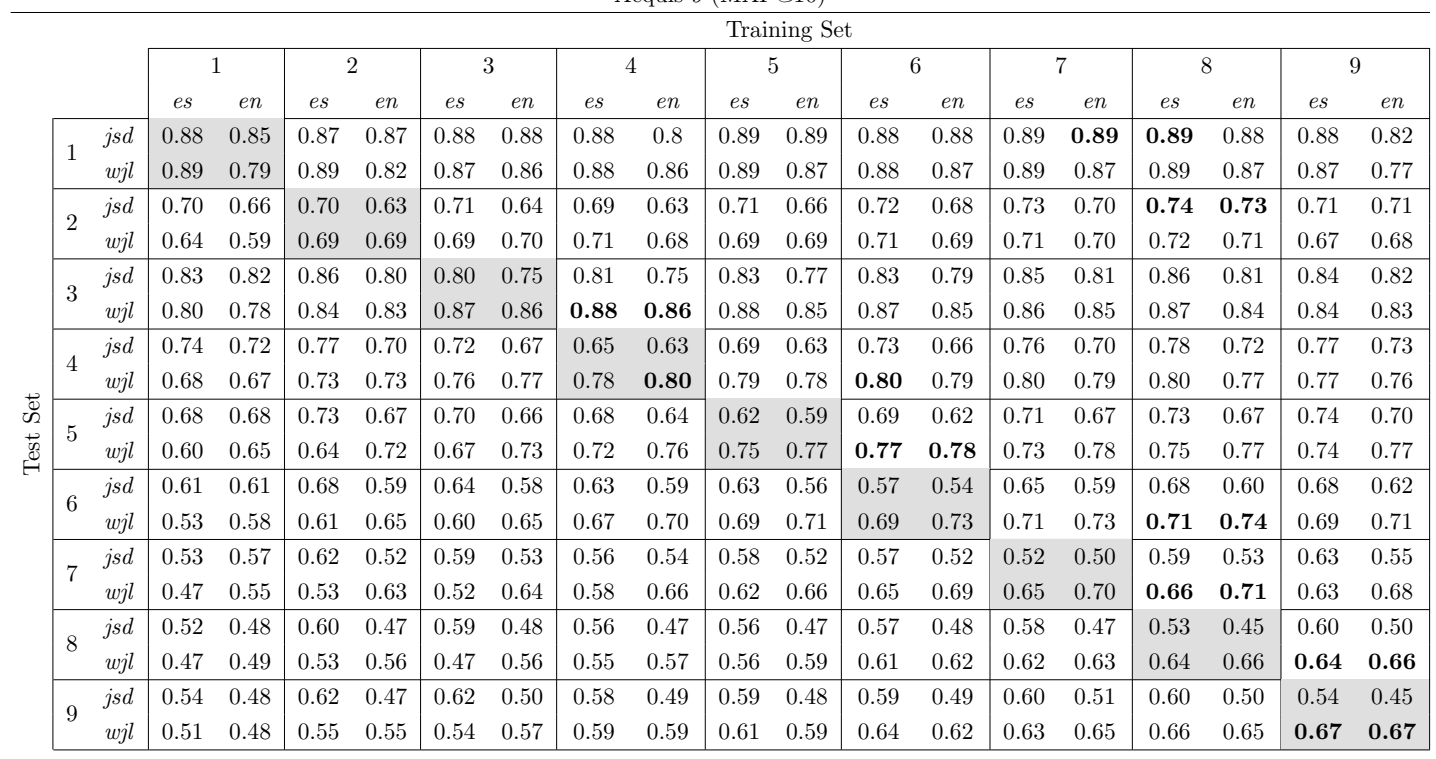

Table 7.10: MAP results by dividing the corpus into nine equal subsets to train and evaluate the models in English(en) and $\operatorname{Spanish}(e s)$

For the same reason, with a small number of dimensions, pairwise comparison is faster using the probabilistic metrics than the hierarchical metrics.

These results reinforce us in the use of probabilistic topic models to facilitate the exploration of large collections of multilingual documents. The knowledge inferred by these models to automatically group semantically related documents is highly sensitive to the texts used in their training. Their ability to generalize such knowledge only seems to make sense in one direction: with texts whose length is equal to or longer than those used during training. This allows us to conclude that, for example, the knowledge extracted from the topics inferred from a collection of tweets (texts of no more than 260 characters), cannot be extended to automatically classify, for example, blog posts (more than 300 characters). If we assume that the complexity of a text increases as its length increases, the logic used to infer topics is unable to capture more complex knowledge than was proposed during training. 


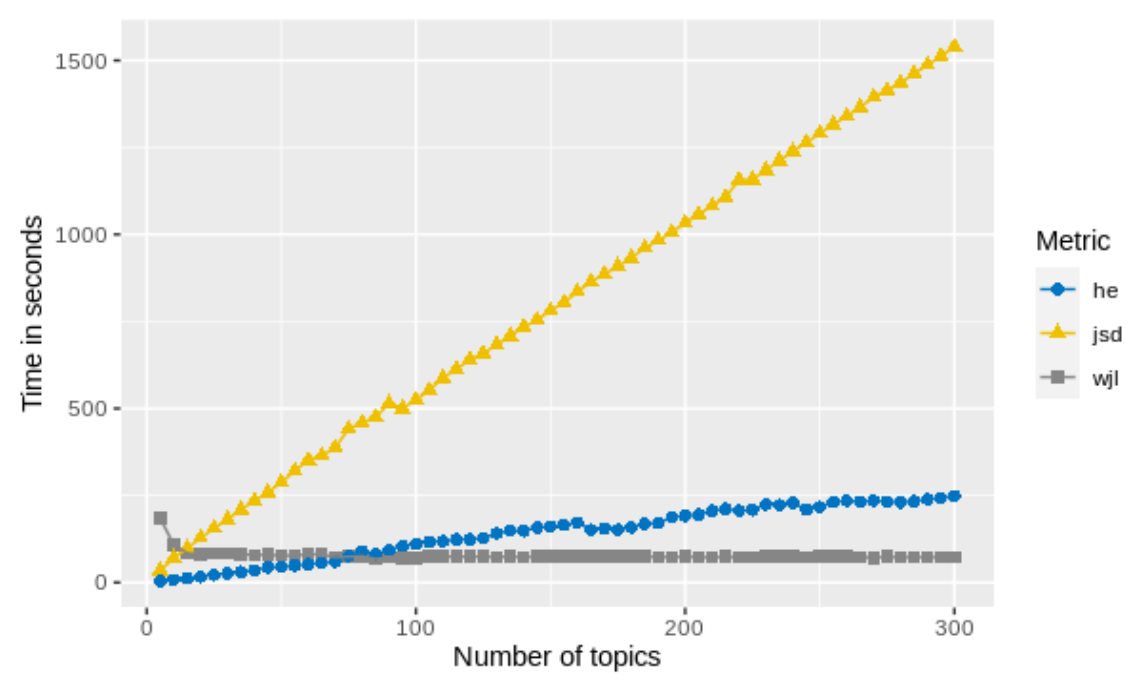

Figure 7.8: Time needed (in milliseconds) to perform the document similarity task using similarity metrics in a corpus of $10^{4}$ synthetic documents

\subsection{Summary}

In this chapter we have described documents based on topic models that create a unique space of representation between different languages. Topics are created independently for each language, and are projected on concepts instead of words. In concept-based representations, documents described by different topic models, with different vocabularies, coexist and can be related since they work on a common representation space. This approach has been evaluated on a multi-language domain and addresses the last research objective of this thesis (R06, define a transformation of the topic-based annotations to create a unique representational space out of the particularities from each language). Representations are analyzed in classification and information retrieval tasks on multilingual document collections. As expected, the performance in terms of accuracy is not as good as that of the approach based on prior knowledge (i.e. topics previously aligned by documents annotated with categories). However, in terms of coverage, the performance of the unsupervised approach is much greater than that offered by the semi-supervised approach, to the point of offering better overall performance (i.e f1) in classification tasks. In addition, the algorithm has proved to perform close to the semi-supervised algorithm in the information retrieval task, which makes us think that the process of topic annotation by set of synonyms should be improved to 
filter those elements that are not sufficiently representative. For example by defining a mechanism, similar to the one used to create "stopwords", to identify "stopconcepts" and avoid using it to describe topics.

In order to perform the evaluations, the new representation system was implemented in our libAIry framework. This extension, together with those described in chapters 5 and 6, cover the last technical objective of this thesis (T04, create a system capable of finding similar documents automatically). It is no longer necessary to translate texts to relate documents from multilingual collections, nor to have annotations beforehand to establish cross-language links or to create aligned models. Documents from different languages are automatically related using their topic distributions created by independently trained models. Each topic model is created from a mono-lingual collection and topics are described by concepts instead of words. In this single conceptual space, texts are represented by hierarchies of concepts based on their topic distributions, and are automatically related according to the concepts they share. The relevance of the shared concepts, according to their topic model, grades the relationship. 


\section{Chapter 8}

\section{Real-World Use Cases}

The hypotheses raised in this thesis have been further tested in projects where its usefulness and domain independence has been proven. The implementation of the proposed algorithms in several systems has allowed third parties to benefit from their results. Some of these projects are framed in the health field, specifically in the area of HIV treatment (Section 8.3) and, recently, in the field of COVID-19 treatment (Section 8.5). In both cases our algorithms have helped to analyze the effects of drugs to treat diseases. There are also projects aimed at measuring the impact of scientific research, at a national level through the analysis of patents and research collaborations (Section 8.2), and at international level by analyzing the originality and creativity of research work (Section 8.1). Finally, the results of this thesis have also been used to facilitate the exploration of public procurement data in Europe (Section 8.4). Tenders by public administrations across Europe were related in an automatic and language-independent way to expand their exploration and allow local administrations to know how similar processes are managed in other countries.

\subsection{Scientific Creativity and Innovation}

Scientific creativity and innovation are key concepts at a time of rapid technological change. Technologies have great potential to supplement human ingenuity in science by overcoming the limitations that people suffer in pursuing scientific discovery. DrInventor 58 proposed an original system to provide inspiration for scientific creativity by

\footnotetext{
${ }^{98}$ http: //drinventor.eu
} 
utilizing the rich presence of web-based research resources (Dong et al., 2017). It is a personal research assistant that informs researchers of a broad spectrum of relevant research concepts and approaches, by assessing the novelty of research ideas, and by offering suggestions of new concepts and workflows with unexpected features for new scientific discovery.

Our topic modeling framework, librAIry (more details in Chapter 4), and the topicbased characterization to measure the similarity between documents (described in Section 5.1.2 powered the DrInventor platform to automatically relate scientific publications from their content. We created a harvester module 99 (Section 4.1.3) that was able to ingest and index research resources from external sources based on the Open Archive Initiative Protocol for Metadata Harvesting100. The resources were processed at different levels of granularity: from the entire documents, to their individual items, parts or even individual words contained in them. On top of those resources DrInventor attached different annotations that further described the instances and gave support to different operations leveraging on them. The model (Figure 8.1) provided a standard way of representing research documents, and was flexible enough to give support to a great variety of analysis techniques bringing value to the information stored in it.

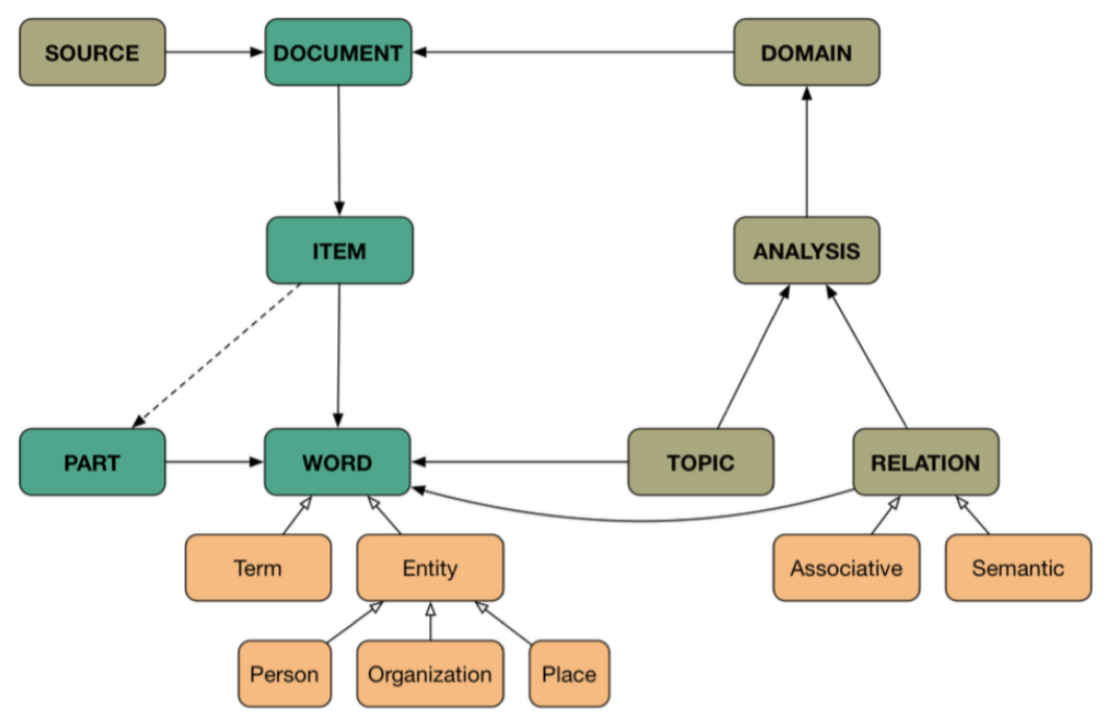

Figure 8.1: Overview of Resources in DRInventor Platform

\footnotetext{
${ }^{90}$ https://github.com/cbadenes/camel-oaipmh

100 https://www.openarchives.org/pmh/
} 
The main types of resources that were considered in DRInventor, from the most fine/grained to the most general ones, were:

- Word: a meaningful element of writing inside a document, composed by a sequence of characters.

- Part: logical division of a document, based on categories of the research discourse such as abstract, introduction, methods, results, conclusions, etc., including also the types of rhetorical sentences (Ronzano and Saggion, 2015) (i.e. approach, background, challenge, future work or outcomes).

- Item: element that make up a research object such as a paper, programmingcode, an image, a workflow, and so on.

- Document: aggregation of items that composes a research object.

- Source: repository where research objects are located. It is used from the platform to automatically retrieve resources.

- Domain: collection of resources created after ingesting the research objects from a repository specified by a source.

- Analysis: execution of an algorithm over a particular domain in the platform. It is responsible for the creation of annotations, such as topics and relations.

- Term: concepts results of the execution of different Natural Language Processing algorithms.

- Entity: named entities such as person, location, or organization.

- Topic: subject that the corpus is elaborating on, such as research areas or trending issues in scientific domain.

- Relation: associative or semantic connection between two resources in a domain.

The DrInventor model served us to refine the model proposed in this thesis (see Section 4.1.1). Some resources have not been addressed (e.g. Source, analysis, topic and relation) to minimize the representation elements to those that can be used to create probabilistic topic models and to calculate similarities between documents (e.g. 
document, domain, annotation). It has also allowed us to generalize our model by adding a snippet resource to represent any element (e.g. part, sentence, entity) that may appear in a document.

\subsection{Innovation and Research in the ICT sector}

In order to take advantage of the overwhelming volume of textual information in electronic format on information and communication technologies (ICT), the Spanish Ministry of Industry developed a plan for the promotion of language technologies. It aims to encourage the development of the natural language processing and machine translation sector in Spain, and to take advantage of these new capabilities to improve public service. In particular, the Corpus Viewer platform ${ }^{101}$ has as main objective to improve the performance of the Ministry in areas such as (1) aid to the ICT sector by identifying similar projects and comparing the different actions, (2) training and employment in the ICT sector by means of studies on the adequacy of training supply and demand in the Spanish ICT labor market, and (3) innovation and research in the ICT sector by means of comparison with other advanced countries and innovation forecasting (Figure 8.2 .
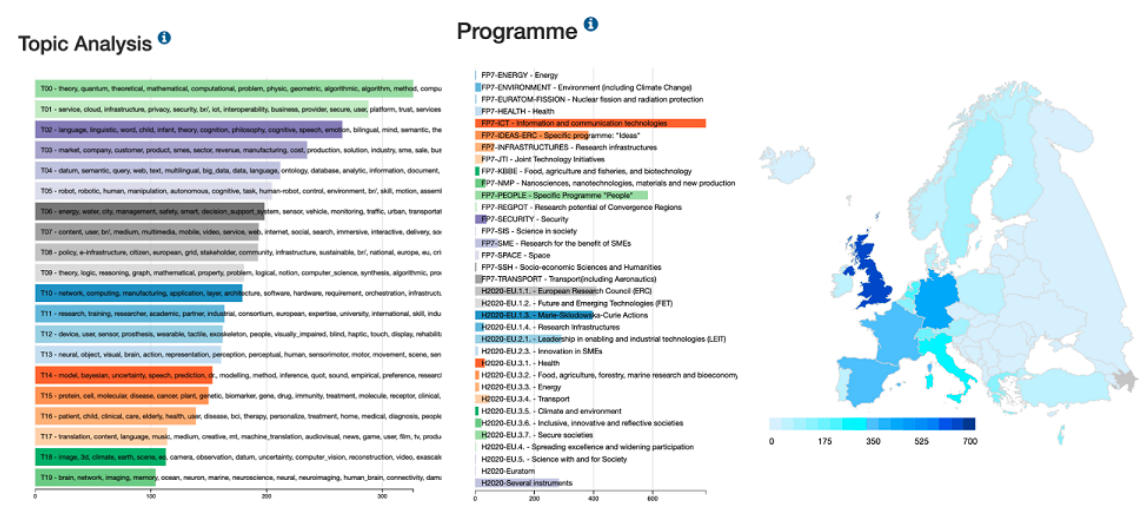

Figure 8.2: The Corpus Viewer platform provides tools for the selection of evaluators or the retrieval of relevant documents (patents, scientific publications, grants and $\mathrm{R}+\mathrm{D}$ proposals for innovation evaluation). In addition, it is used for plagiarism detection, identification of double funding cases and fraud in aid grants and proposals submitted for national funding.

\footnotetext{
${ }^{101}$ https://www.plantl.gob.es/tecnologias-lenguaje/actividades/plataformas/Documents/ corpus-viewer/manual-corpus-viewer-en.pdf
} 
Three document collections were available for the project: a small-size collection of around 70 thousand technical and administrative texts about Spanish R\&D projects; a medium-size collection with more than 500 thousand descriptions of R\&D projects funded by the European Union retrieved from the CORDIs 102 dataset; and a large-size collection created from UPSTO ${ }^{103}$ with more than 32 million American patents.

Our contribution in this project through the framework librAIry (see Chapter 4), that covers the complete life cycle of probabilistic topic models, and the algorithms that build topic hierarchies based on relevance (see Chapter 5) and compare texts efficiently from topic distributions (see Chapter 6) served to identify the main themes and to semantically relate the texts of the documentary collections (Samy et al., 2019).

Multiple topic models were created for each documentary corpus, with different configurations (i.e. number of topics, Part-of-Speech filters, lemmas). For each configuration, the texts were indexed, through an inverse index, in databases where they were also annotated with the topic hierarchies and the similarities between their texts were calculated from these topic-based representations. All this information was offered through a Restful API to a web interface where the documents were linked in a network. The source code of the algorithms implemented in the system, the models and the data are publicly available for reuse 104 .

The platform is currently used by $\mathrm{R}+\mathrm{D}$ policy makers, $\mathrm{R}+\mathrm{D}$ program managers and coordinators (policy implementation), grant and aid evaluators, and researchers and public research organizations as well as companies. The most common use cases are: (1) public policy design, (2) management of calls through classification of applications, assignment of evaluators, similarity of documents, estimation of innovation, and evaluation and selection of applications, (3) monitoring the results of the intervention and measuring its impact and (4) alarms for detection of plagiarism and fraud patterns.

\subsection{Polypharmacy and Drug-drug Interactions}

Does the number of concomitant drugs in people living with Human Immunodeficiency Virus (HIV) increase with age and is it greater than in non-HIV-infected persons? This research question (López-Centeno et al., 2019) seems to be far from the scope of this

\footnotetext{
102 https://cordis.europa.eu

103 https://wWw.uspto.gov

104 https://github.com/cbadenes/corpus-viewer-addons
} 
thesis. However, if we take into account that concomitant drugs are the drugs that a patient also uses to treat other diseases, we can draw an analogy to bring it closer to our domain. It aims to analyze patients from the medicines they receive, and our algorithm organizes documents described by topics. A patient, seen as a document, is described by the drugs he/she receives to treat HIV, which can be represented as topics, and the drugs he/she receives to treat other diseases (i.e concomitant drugs), which would be used to set the relevance of each topic from their interactions. The relationships between patients depend on the drugs they share, and can be measured by the topics they share when they are represented as documents.

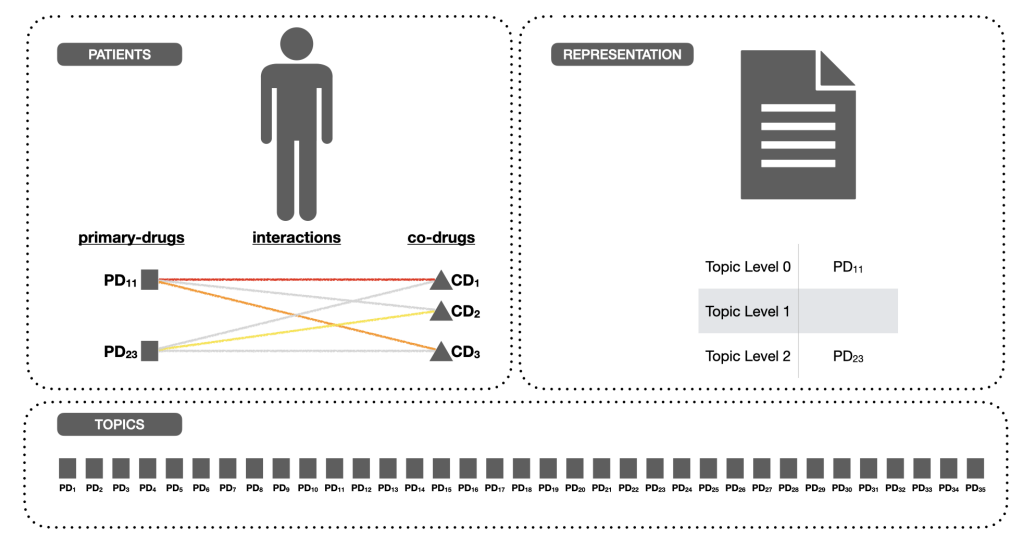

Figure 8.3: Representation of patients through topic hierarchies based on the interactions between primary-drugs and co-drugs

Specifically, in this work we were able to put into practice our approach to make comparisons (Chapter 6) when discovering drug-drug interactions (DDIs) in HIV patients. The anonymized registries from the Madrid Health Service (SERMAS) database were used to build a working database with information about all patients who picked up antiretrovirals (ARVs) or non-HIV medications during the study period. A total of 22,945 people living with HIV and 6,613,506 individuals without HIV had received medications. Medications from the SERMAS database were separated into ARVs and non-HIV drugs: 35 primary-drugs (i.e ARVs medications) and 1,058 co-drugs (i.e nonHIV medications). All drug pairs between primary- and co-drugs were interrogated using the University of Liverpool (UoL) Drug Interactions Databas ${ }^{105}$, with more than

\footnotetext{
105 https://www.hiv-druginteractions .org
} 
24,000 HIV DDIs between ARVs and non-HIV medications, to generate a comprehensive list of potential DDIs. The Liverpool flag classification categorizes the severity of DDIs as follows: a red flag indicates medications that should not be coadministered as they might lead to serious adverse events or profoundly affect antiretroviral therapy efficacy; an orange flag indicates a potential interaction that might require dosage modification or close monitoring to minimize clinical consequences; a yellow flag indicates a potential interaction of weak relevance not requiring additional monitoring or dosage adjustment; a green flag indicates no anticipated risk of inter-action; and a gray flag indicates no clear data are available to assess whether a DDI will occur.

We built a representation system where each primary-drug was represented as a topic, and each patient was described as a document containing those topics with different weights (Figure 8.3). This value depends on the DDIs between the primarydrugs and the co-drugs of the patients. Red-flag interactions correspond to the most relevant topics (i.e. level 0), orange-flag interactions correspond to the following topics (i.e. level 1), and yellow-flag interactions correspond to the least relevant topics (i.e. level 2). Primary-drugs are assigned to the most relevant level. Patients are thus described by DDIs, making it easy to identify sets of risks according to the severity of the interactions.

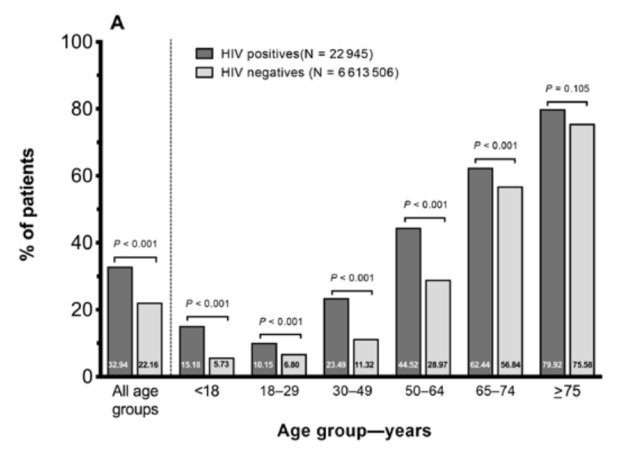

Figure 8.4: Distribution of polypharmacy among people living with and without HIV according to age (López-Centeno et al. 2019$)$.

Our contribution facilitates the analysis of patients and drugs (interactions) without having to make all the comparisons between them (Figure 8.4). Patients are grouped by interactions on the same primary medications. The source code we have developed 
to discover drug interactions is publicly available for reuse 4 , but for privacy reasons we cannot publish the code to manage patients.

\subsection{Public Procurement Data}

In order to improve public procurement in European Union (UE), TheyBuyForYou (Soylu et al. 2020) aimed to provide high quality, open, and integrated procurement data. The lack of common agreement across the EU on the data formats for exposing such data sources and on the data models for representing such data, leads to a highly heterogeneous technical landscape. In this context, a knowledge graph (KG) based platform and end-users tools have been created and publicly released for integrating and reconciling cross-border and cross-language procurement and company data. But procurement processes are not only creating structured data, but also constantly creating additional documents (tender specifications, contract clauses, etc.). These are commonly published in the official language of the corresponding public administrations. Only some of these, for instance those published in TED[107, are multilingual, but the documents in the local language are typically longer and much more detailed than their translations into other languages.

A civil servant working at a public administration on a contracting process may be interested in understanding how other public administrations in the same country or in different countries (and with different languages) have worked on similar contexts. Examples may include finding organizations related to a particular procurement process, or search for tenders related to given procurement text. Based on our results to create language-independent probabilistic topic models (Chapter 7), we worked on an cross-lingual search engine 108 that provides support to these types of users, with the possibility of finding documents that are similar to a given one independently of the language in which it is made available (Figure 8.5). We also created a Jupyter notebook with some representative examples to facilitate its use 109 .

The document search engine is based on the use of cross-lingual labels created from sets of cognitive synonyms (synsets) and unsupervised probabilistic topic models. The

\footnotetext{
${ }^{106}$ https://github.com/cbadenes/hiv-ichart-client

${ }^{107}$ https://ted.europa.eu

${ }^{108}$ https://tbfy.librairy.linkeddata.es/search-api

109 http://bit.ly/tbfy-search-demo
} 


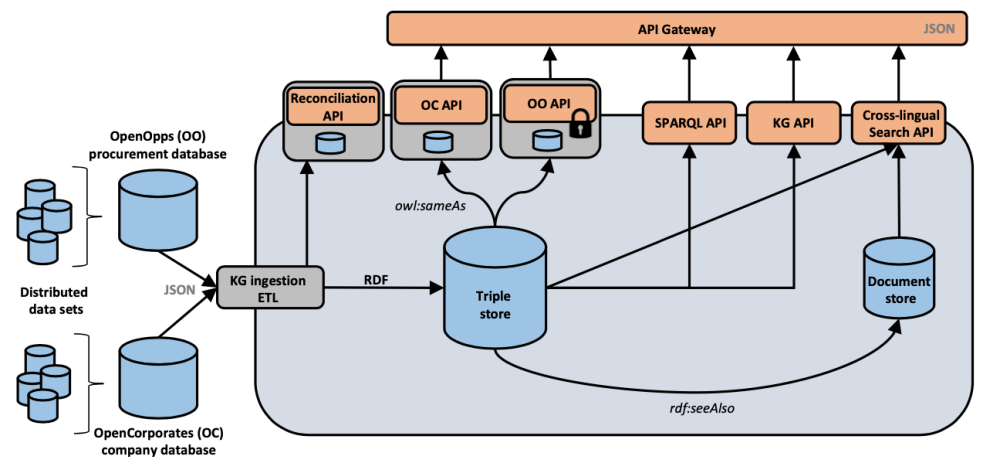

Figure 8.5: High-level architecture of the TheyBuyForYou platform (tbfy.eu)

original low-dimensional latent space created by probabilistic topic models BadenesOlmedo et al. (2019b) was extended with two new languages. In addition to the original English, French and Spanish models, we created Portuguese and Italian models to increase the common space shared by all languages (see Section 7.1.3). Topics were described by cross-lingual labels created from the list of concepts retrieved from the Open Multilingual WordNet. Each word was queried to retrieve its synsets. The final set of synsets for a topic was the union of the synsets from the individual topwords of a topic. Documents were then represented as data points and transformed from the original feature space based on mono-lingual topic distributions into a crosslingual hierarchical-based space, so that similar data points share relevant cross-lingual concepts (see Figure 8.5). Since topic models create latent themes from word cooccurrence statistics in a corpus, a cross-lingual topic specifies the knowledge about the word-word relations it contains for each language.

The data and platform components were made available openly for the community to contribute and use; a catalogue is available online ${ }^{110}$ with pointers to the code repositories, online versions of the artefacts, and relevant documentation

\subsection{COVID-19 and Coronavirus-related Research}

In the absence of sufficient medication for COVID patients due to the increased demand, disused drugs were employed or the doses of those available were modified by hospital pharmacists. Some evidences for the use of alternative drugs can be found in the

\footnotetext{
${ }^{110}$ https://tbfy.github.io/platform
} 
existing scientific literature that could assist in such decisions. However, exploiting large corpus of documents in an efficient manner is not easy, since drugs may not appear explicitly related in the texts and could be mentioned under different brand names. New experiments and results are continually being published, and people in charge of clinical protocols cannot keep up to date with all of them. This situation calls for solutions that help health care providers and researchers easily extract such knowledge from the enormous scientific corpus that is being created.

The Allen Institute for Artificial Intelligence created the COVID-19 Open Research Dataset (CORD-19) (Wang et al., 2020). It is a continuously growing corpus with all publicly available COVID-19 and coronavirus-related research (e.g. SARS, MERS, etc.) in the last fifty years. This dataset can be used as a source of information to extract knowledge related to the disease. At the time of our study, it was composed of more than 60,000 scientific articles: 23,428 open access articles from PubMed Central, 35,240 research articles from a corpus maintained by the World Health Organization (WHO), and 1,945 bioRxiv and medRxiv pre-prints.

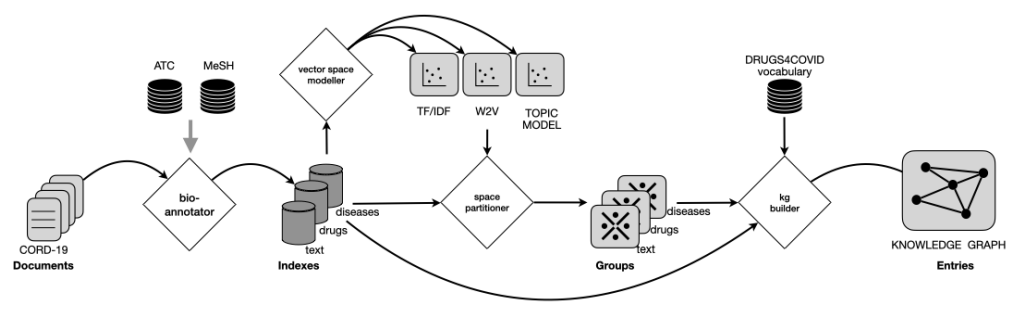

Figure 8.6: High-level workflow of a search engine and a knowledge graph through annotations created from the CORD-19 dataset

Drugs4Covid ${ }^{111}$ (Badenes-Olmedo et al. 2020a) arises to enable a drug-oriented exploration of large medical literature as available in CORD-19 dataset. It is an initiative of the Ontology Engineering Group ${ }^{112}$ (OEG) that combines natural language processing with word embedding techniques and knowledge extraction technologies to provide doctors and medical researchers with tools that make their work easier by structuring the information contained within the papers. Our contribution in this work was to use the librAIry framework (Chapter 4 ) to process and annotate the scientific publications, together with the use of our representation algorithms based on topic hierarchies

\footnotetext{
$1 1 1 \longdiv { \text { http://drugs4covid.oeg.fi.upm.es } }$

${ }^{112}$ https://oeg.fi.upm.es
} 
(Chapter 5) and the application of similarity metrics and hash functions (Chapter 6) to automatically relate texts. The final goal is to automatically extract, organize and publish the drug-oriented knowledge from the medical literature needed to answer common questions posed by domain experts such as What are the effects of using chloroquine and hydroxychloroquine to treat COVID-19 patients? In which experiments have mefloquine and azithromycin been used and related to which diseases?.

More than 60k scientific papers were analyzed from the CORD-19 dataset available on April 2020. Around 5M sentences and 2M paragraphs were annotated with the drugs and diseases mentioned in them. A total of 6,400 diseases and 2,400 drugs were characterized to enrich the searches on the corpus and the knowledge graph. We created vectorial representations of each of the articles using techniques based on word embeddings and probabilistic topic models. Then, diseases and drugs were related to each other and suggested drugs that may be considered as replacements for others. An open catalogue of drugs was created and results were publicly available through a drug browser, a keyword-guided text explorer, and a knowledge graph. The tools created from the proposed workflow help to quickly create domain-specific search engines and knowledge graphs (KG) (Fig. 8.6) over any corpus of scientific documents. Such techniques can be reused for other similar crisis in the future, and in any other situation where these tools could be valuable. The methods and services are available openly for reuse ${ }^{113}$, and will continue to be improved in 2021 and 2022 with funding from FundaciónBBVA 114 .

\footnotetext{
113 https://librairy.github.io/covid19

${ }^{114}$ https://www.fbbva.es
} 


\section{Chapter 9}

\section{Conclusions and Future Work}

This thesis addresses different challenges to facilitate the exploration of large multilingual document corpora through the use of probabilistic topic models. Four main research problems rise from the use of topic models, as we discussed in Section 3.2. The first one is the efficiency to create and infer probabilistic topics. There are some technical barriers that may limit a wider use of topic models in high-volume scenarios. The second challenge is the explainability of topic-based relations among documents. It is difficult to understand how two documents are related from the numerical distance of their vector representations. The third challenge is reducing the comparison complexity among topic distributions at large-scale. Brute-force approaches are not feasible for big documentary corpora. And finally, the fourth challenge is multilinguality when comparing topic distributions from documents written in different languages. In order to be able to work with multilingual corpora at any scale, it is necessary to avoid the need for translators or annotations between languages that may limit their feasibility.

As shown in this thesis, the main hypothesis has proven to be true, so large multilingual document collections can be automatically analyzed to discover thematic representations that enable an exploration through related texts (H1). Based on the evaluation of our results, probabilistic topic models enable an unsupervised exploration of corpora containing a huge number of texts written in different languages. Below we review the research problem mentioned above through the hypotheses evaluated in this work and the contributions we offer to address them. The rest of the section discusses the impact, current limitations and future lines of our work. 


\subsection{Contributions}

The main technical contribution of this thesis is the librAIry framework, a system that processes and analyzes large collections of text resources creating and using probabilistic topic models. librAIry encompasses several scientific and technical contributions that are aimed at addressing the four aforementioned research problems. Following the methodology described in Section 3.3 , we have evaluated our hypotheses using librAIry for large multilingual document corpora.

\subsubsection{Efficient Creation and Use of Probabilistic Topic Models}

As discussed in Chapter 4 , previous works dealing with probabilistic topic models are mostly focused on improving the learning process (i.e. accuracy, coverage) and ignore other important features related to its development in potentially heterogeneous scenarios. The creation of a topic model (Fig. 4.1) covers a first stage of document preparation, where texts are pre-processed to create bags-of-words. The next stage (training stage), builds a model based on patterns among word distributions. The model is then packaged for distribution in the (publication stage). And finally the model can be used and reused in the (exploitation stage). Our objective in this thesis is to facilitate the creation of reusable probabilistic topic models by minimizing their technical dependencies for use in both large- and small-scale contexts. In our analysis of the state-of-the-art we identified some research challenges that make it difficult to achieve that goal: reuse of topic models is limited by incompatibility problems (RCInterface1), and there is no unified or standardized format for distributing topic models (RCInterface2). Along these research challenges we formulated one hypothesis and proposed a series of contributions that we discuss below.

H1.1 Documents can be efficiently annotated at a large scale by distributing across different computation nodes both natural language processing tasks and topic models. This hypothesis is motivated by the limitations of existing works identified and discussed in Section 2.4.1 that can be summarized as follows:

Limitation 1: Summaries or sections are usually considered to describe documents using probabilistic topics due to technical difficulties in processing full-texts in large 
collections. However, mining full-text articles gives consistently better results (Westergaard et al., 2017).

Contribution 1: We have created librAIry, a topic modeling framework described in Section 4.1 to overcome this limitation. This framework has been validated in real world scenarios with large document collections. librAIry uses an event-driven architecture for text processing and topic inference that adapts its workload to the size of the corpus. Initially, data were organized into snippets (to describe pieces of texts), documents (to represent full texts), and domains (to group documents), but it evolved to a more reduced representation of texts and collections. librAIry has been tested as part of the Corpus Viewer platform (Samy et al., 2019), where it was used to analyze the current situation and trends of information and communication technologies (ICT) through the study of patent collections and grants for R\&D projects (see Section 8.2). We have also used this implementation to support complex calculations on data sets from different domains. For example, to relate patients according to the medicines they receive (López-Centeno et al. 2019) (see Section 8.3), or to relate medicines or diseases from the experiments where they are used (see Section 8.5).

Limitation 2: APIs based on topic models define their own distribution formats limiting the interoperability of the models (Lisena et al., 2020). The efforts made so far in the state-of-the-art do not propose a unified model to exchange topic models, understood as an already accepted standards-based format.

Contribution 2: Our second contribution consists of a method to make topic models openly accessible as web resources. In Section 4.2 we propose a web service template based on REST principles to homogenize the format of topic models and facilitate their usage. This allows easy accessibility and reuse of topic model related information for both humans and machines. As described in Section 4.2.1 three tasks guide the creation of a topic model as web service: reproducibility, exploration and inference. The list of available methods for using a topic model is provided in Table 4.2. Finally, an online repository has been also proposed in Section 4.2 .2 to promote the reuse of existing models as virtual services that have meta-information about the training process with which they were created and multiple versions. 


\subsubsection{Explainability of Topic-based Relations among Documents}

As discussed in Chapter 5, state-of-the-art metrics for comparing topic distributions are difficult to understand. Since topic distributions are density functions, the distance between every pair of documents is calculated by aggregating the intersections of each dimension of the vector, i.e., of each topic. However, as seen in Section 2.4.2, highly dimensional models create more specific topics than models with fewer dimensions, and the topic specificity influences the way in which topic distributions are related, and consequently how documents can be related. The distance between the same two documents may vary when using topic models with different dimensions (Figure 2.3). Our objective in this thesis is to describe texts based only on the most representative topics and compare documents taking into account these descriptions. But we have identified some research challenges that make it difficult to achieve that goal: there is no common criteria for identifying the most representative topics in a document (RCExplainable1), it is difficult to understand the distance between topic distributions (RCExplainable2) and there is no common criterion for determining whether two documents are related (RCExplainable3). Along these research challenges we have formulated one hypothesis and proposed a series of contributions that we discuss below.

H1.2 It is possible to semantically relate documents by comparing their most relevant topics. This hypothesis is motivated by the limitations of existing works identified and discussed in Section 2.4.2, which can be summarized as follows:

Limitation 3: We analyzed the behavior of topic distributions when using topic models with different dimensions, i.e. different number of topics. Our evaluations conclude that the most relevant topics cannot be identified by fixed thresholds, since the dimensions of the model vary the relative distances also vary. Nor can they be identified using clustering techniques based on centroids, since the number of groups with homogeneous weights is unknown a priori.

Contribution 3: We proposed a method to identify the most relevant topics that takes advantage of a particular behavior of Dirichlet distributions describing topic distributions. Since the highest weighted topics have a high influence on the rest, the most representative topics can be calculated by comparing the sum of their weights with respect to the rest. Our method Cumulative Ranking on Dirichlet distributionbased Clustering (CRDC) is based on the cumulative sum of the weights of the highest 
topics (Figure 5.14). The number of relevant topics is dynamically determined by a threshold, and once this threshold is reached no more topics are considered.

Limitation 4: Explore the knowledge inside document collections requires to calculate a similarity matrix with all possible comparisons between elements, so we can later select the most pertinent ones. Computing a $n \times n$ matrix takes $O\left(n^{2}\right)$ time, and obtaining all possible pairs of similarities in a large collection of documents can be unfeasible because of the quadratic cost of comparing every pair of elements.

Contribution 4: We propose a novel clustering technique based on topic model distributions that reduces the complexity to find relations between documents in a large corpus of textual documents, without compromising efficiency and providing additional information about relations. The results of our approach are competitive against both a centroid-based and a density-based clustering baselines, as described in Section 5.2.2. While $K$-Means takes $O\left(n^{k} * \log n\right)$ and $D B S C A N$ takes $O(n * \log n)$ time to classify $n$ documents in a collection, the proposed algorithms only take linear time $(O(n))$ because they do not require any other data except their own topic distribution to assign it to a cluster.

\subsubsection{Complexity Reduction in Comparisons among Topic Distribu- tions at Large-Scale}

As discussed in Chapter 6, brute-force techniques cannot be applied to compare all items in a large corpus. Document similarity comparisons are too costly to be performed in big collections of data and require more efficient approaches than having to calculate all pairwise similarities. Hashing is one of the popular solutions for approximate nearest neighbours (Zhen et al., 2016), due to the low storage cost and fast retrieval speed. However, existing hashing methods for probability distributions only focus on the efficiency of searches from a given document (Mao et al., 2017), without handling complex queries or offering hints about why one document is considered more similar than another. Our objective in this thesis is to find documents with similar topic distributions without calculating all pairwise comparisons and without discarding the notion of topics from their representation. But we have identified two research challenges that make it difficult to achieve that goal: there are no mechanisms that efficiently partition the topic-based search space without compromising the ability for thematic exploration (RCComparison1), and there are no similarity metrics that 
compare partial distributions of topics (RCComparison2). Along these research challenges we have formulated one hypothesis and proposed a series of contributions that we discuss below.

H1.3 Dividing the representational space into regions based on topics and relevance levels allows searching for related documents without having to calculate all pairwise comparisons and without discarding the notion of topics for further processing. This hypothesis is motivated by the limitations of existing works identified and discussed in Section 2.4.3 that can be summarized as follows:

Limitation 5: Our CRDC approach to identify the most relevant topics depends on the manual tuning of a hyperparameter, the threshold, that helps identify relevant documents. Moreover, this method does not measure degrees of similarity since it only establishes whether two documents are related or not.

Contribution 5: We created a new data structure to represent topic distributions as topic hierarchies that use the relevance of each topic to define hierarchy levels. This representation has also helped to understand why two documents are similar, based on the intersection of topics at hierarchies of relevance. The approach can accommodate additional query restrictions when searching for related documents (e.g. documents that mainly deal with one theme, although they also deal with another) and has proven to obtain high-precision results.

Limitation 6: State-of-the-art distance metrics among topic distributions are based on density functions, not on sets of topics according to their relevance. The representation of documents in these cases is not based on weighted vectors, but on sets and levels. Hence distance metrics based on density functions are not applicable.

Contribution 6: A new similarity metric is proposed based on the most relevant topics. The distance between two texts is proportional to the number of topics they share at the same relevance level. The performance of the metric was evaluated in unsupervised classification tasks and shows (Tables 5.3, 5.2, 5.4 and 5.5) results with high precision and recall values. The corpus used was the JRC-Acquis with annotations in EUROVOC categories.

Limitation 7: Approximate nearest neighbours methods based on probability distributions, i.e. topic models, are not able to organize documents (1) by subject areas or 
(2) by levels of similarity, nor do they offer (3) an explanation of the similarity obtained beyond the vectors used to calculate it.

Contribution 7: We developed a method to compare and organize huge document collections based on similar topic hierarchies. The hierarchy levels are compared and the distance between texts depends on the degree of intersection between pair of representations. In addition, the technique to represent and compare documents has been implemented in our librAIry framework.

\subsubsection{Multilinguality through Monolingual Topic Models}

As discussed in Chapter 7, documents in different languages must be described by multilingual topics to be thematically related without having to translate their texts. Topic models emerge as distributions over crosslingual equivalence classes of words and require document-aligned or theme-aligned corpora, or they are based on word alignments from bilingual dictionaries. Some approaches propose to translate only the words used to characterize the topics across the languages, such as anchor words (Yuan et al., 2018) or top words (Yang et al. 2019). Others are placed between word and document alignments and propose crosslingual topic models using language-independent categories from external resources (Piccardi and West, 2020$)$. However, the requirement of parallel/comparable corpora or dictionaries limits the usage of these models in many cross-lingual situations. Our objective in this thesis is to find cross-lingual representations of documents that keep the notion of topics, independently from the language used, in order to draw relations between them. But we have identified a research challenge that makes it difficult to achieve that goal: there are no approaches to abstract probabilistic topics in language-independent spaces without translating texts or aligning documents (RCCrossLingual1). Along this research challenge we have formulated one hypothesis and proposed a contribution that we discuss below.

H1.4 It is possible to relate documents in different languages without having to translate them, by using language agnostic concepts from their main topics. This hypothesis is motivated by the limitations of existing works identified and discussed in Section 2.4.4 that can be summarized as follows:

Limitation 8: Existing methods based on bag-of-words representations require prior knowledge between languages to create topic models that represent documents in a common, language-independent space. They can be dictionaries to make translations, 
shared categories across languages or reference terms to align language-independent representations.

Contribution 8: We propose an unsupervised technique for building cross-lingual topic models based on sets of cognitive synonyms (synsets) (Miller, 1995) to discover relations between language-specific topics once the models (for each language) have been created. This technique does not require parallel or comparable data for training (Badenes-Olmedo et al., 2019a b). A topic-based space is created across languages based on language-dependent topic models that have been independently created. This representational model can be used for large-scale multilingual document classification and information retrieval tasks. In addition, the algorithm proved to perform close to the semi-supervised algorithm (i.e. Labeled LDA (Ramage et al., 2009) ) in an information retrieval task, which makes us think that the process of topic annotation by sets of synonyms (i.e. concepts) can be improved to filter out those elements that are not sufficiently representative.

\subsection{Impact}

In addition to the aforementioned contributions, the technologies and techniques introduced in this thesis and their deployment in some practical use cases (as described in Chapter 8) have had a positive impact on the way users browse document collections on a large scale.

First, access to probabilistic topic models via REST APIs has increased thanks to librAIry as an open source framework modularly distributed via virtual containers. Since March 2016, taking into account statistical data gathered from the DockerHub platform 115 where the librAIry services images reside, the download traffic is as follows:

- the module 'librairy/modeler-topics-service' that builds topic models from CSV files or Solr indexes has been downloaded more than 1,200 times. One third may be self downloads/rebuilds due to new versions.

- the module 'librairy/api' that annotates documents with probabilistic topics created with the previous module has been downloaded more than 1,100 times.

${ }_{115}^{15}$ https://hub.docker.com/orgs/librairy/repositories 
- the 'librairy/nlp' module that generates bags of words, extracts lemmas and annotates Part-of-Speech from texts in English, Spanish, French, Italian and Portuguese has been downloaded 373 times.

- the 'librairy/search-api' module that relates documents from their topic distributions across multiple languages has been downloaded 212 times.

- the 'librairy/explorer' module to browse via Web among the relations found in document collections was downloaded 327 times.

More importantly, among those that publish a topic model via REST API, the modules 'librairy/openresearch-model' (created from the OpenResearch corpus) with 612 downloads and the 'librairy/dbpedia-model' (created from a subset of DBpedia entities) with 164 stand out, while the rest of the models (e.g. 'librairy/jrc-en-model', 'librairy/ods-model', or 'librairy/lynx-model', among others) are between 20 and 40 downloads or so.

\section{UNIQUE VISTTORS PER DAY - INCLUDING SPIDERS}

HIS HAVING THESAMEIP, DATEAND AGENT AREAUNIQUEVIST

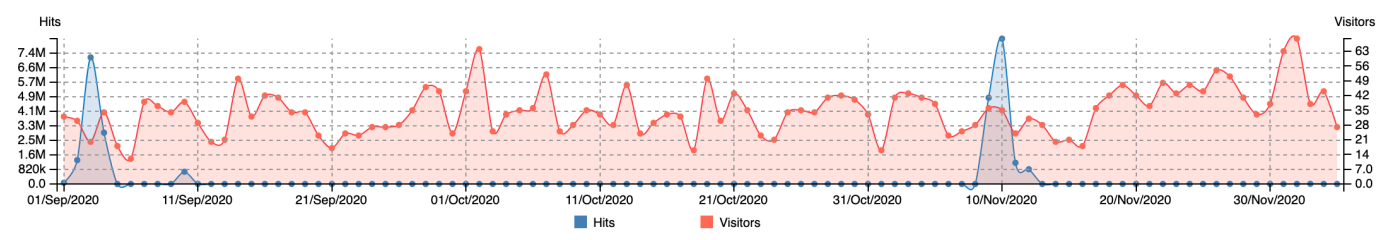

Figure 9.1: librAIry API usage statistics from September 2020 to February 2021

Along with the reuse statistics provided by librAIry, we also have usage statistics of services deployed using the technology proposed in this thesis. During the time period from September 2020 to February 2021 we logged accesses to the librAIry AP] $]^{116}$ that supports, among others, a cross-lingual search engine for exploring public procurement data in the European Union (described in Section 8.4), and a scientific publication browser for exploring drugs on COVID-19 treatment (described in Section 8.5). A total of 42,122,078 requests (Figure 9.1) from 2,734 different users, taking unique IPs into account, were supported. In addition, access from different operating systems shows that it is advisable to use Web access interfaces to exploit these resources (Figure 9.2).

\footnotetext{
${ }^{116}$ http://librairy.linkeddata.es/api
} 


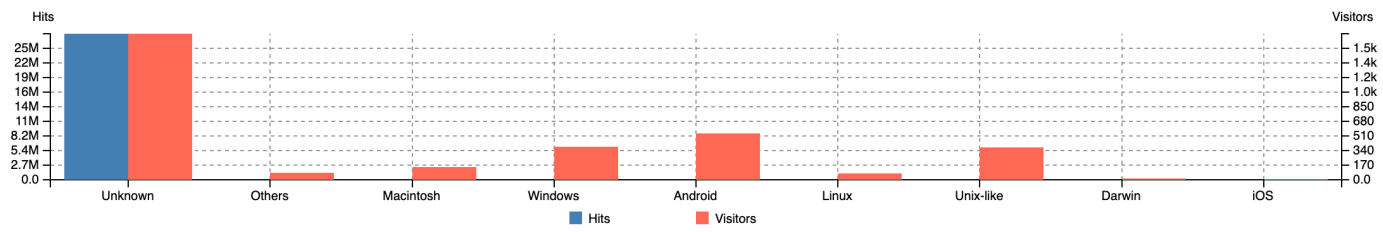

Figure 9.2: use of librAIry resources from different operating systems from September 2020 to February 2021

Second, some of the methods and techniques proposed in this thesis have been used in recent work by other researchers. The efficient topic modeling framework described in Section 4.1 was used, among others, to create, publish, distribute and reuse probabilistic topic models for studing the Language Technologies sector in Spain (Samy et al. 2019). The analysis took into account the structured and unstructured data from the ACL Anthology repository in order to portray the current panorama in terms of underlying topics and their evolution in recent years in comparison with the international community. The framework has also been used to facilitate the classification of public offering using big data techniques (Herranz and Arenas-Garcia, 2019) and to analyze texts in reading support systems (Gómez-Carpintero, 2020). Our methods to categorize documents through topic hierarchies and to make efficient large-scale comparisons described in Sections 5.2 and 6.1 have been tuned out to be used in different domains. In (López-Centeno et al., 2019), the algorithms were adapted to compare patients from representations based on the medications they were using. The approach assumes drugs, like topics, can be distributed in different proportions according to the patient. To take advantage of the hierarchical representation of the topics, a more complex approach based on drug-drug interactions was performed (see Section 8.3).

Finally, thanks to the technologies developed in this thesis, a spin-off in the field of natural language processing and knowledge management has been recently founded. librAIry S.L ${ }^{117}$ was created at the beginning of 2021 to support, among others, the following challenges:

- Corpus Exploration: By knowing how topics are present in a corpus in terms of relevance, it is possible to derive a thematic network where the nodes represent

\footnotetext{
${ }^{117}$ http://librairy.eu
} 
the different topics and the edges are the documents containing them on the same level of relevance. Thus, the higher the number of common documents the stronger the connection would be between two topics. This kind of network would help users to see how different topics are connected with each other in a corpus, facilitating the exploration between topics.

- Topic Discovery: Commonly occurring topics, when supported in many documents, may become valuable topic candidates themselves. Documentary management system may offer these potentially new topics to users to include them in their corpus description.

- Language Abstraction: Approaches like (Hao and Paul, 2018) create multilingual topics from any corpora. These approaches can benefit from our hierarchical representations to create language-independent spaces where documents are described by their most relevant topics.

- Corpus Visualization: As stated in Section 6, one of the reasons why groupings are created is for summarization and organization purposes. Hence, commonly occurring topics by hierarchies can be used to simplify the complexity of a corpus by collapsing their documents under a single group. If a corpus has overlapping clusters it would be possible to create different views according to user preferences, simplifying the overall complexity shown to the final user.

- Corpus Compression: Similarly, if several documents share the same most relevant topics, it would be possible to store the common relevant topics instead of every unique topic distribution, for efficiency. This would be particularly useful when dealing with similar or identical texts.

- Topic Suggestions: Commonly relevant topics may be used to suggest how a user may complete the writing of a document. By comparing the current topic hierarchies with the commonly relevant topics, it would be possible to recommend the next topic o topics to be considered in the document.

- Document Ranking: Once the topics are described by hierarchies of relevance, it would be possible to order the documents in a corpus by different criteria and create rankings. Possible examples of rankings are basic searches based on one or 
several highly relevant topics, or more complex searches that combine different topics and different degrees of relevance.

\subsection{Future Directions}

The work presented in this thesis provides contributions that enable large-scale browsing of multilingual document collections guided by their main topics, and deploy probabilistic topic models as web services. Moreover, our work contributes to the application of semantic technologies to organize texts without the help of domain experts and provides evidence of their broad impact through the real-world use cases presented in Chapter 8. Nevertheless, we have highlighted some limitations, open issues and new research directions throughout the thesis. In this section, we discuss and outline future directions in relation to our contributions. We consider the four research dimensions proposed in Chapter 3 to organize the discussion: i) efficiency, ii) explainability, iii) complexity, and iv) multilinguality

\subsubsection{Efficiency in Learning Models}

In Chapter 4 we have proposed the librAIry framework to create probabilistic topic models and annotate document collections at large-scale. Data is organized in texts to reflect parts or pieces of texts or to represent full texts, and domains to group documents. Processing tasks are distributed over these resources among software modules connected through REST APIs, even the topic models. In this thesis, we have demonstrated their suitability for large corpora through some real world scenarios such as DrInventor, TheyBuyForYou or Corpus Viewer. librAIry has proven to be a valid and scalable text processing framework. However, there are several open issues, challenges and interesting future lines of research that we ellaborate below.

First, we have demonstrated the applicability of using topic models and natural language processing techniques via REST APIs, but the current trend based on Python prototypes uses non-exportable (i.e from other programming languages) models from online repositories (e.g. huggingface). The ONNX (Open Neural Network eXchange) and ONNX Runtime (ORT) projects, for example, are an effort from leading industries in the AI field aiming to provide a unified community-driven format to store and efficiently execute learning processes leveraging a variety of hardware and dedicated 
optimizations. But given the high resource requirements for working with increasingly heavy models (e.g. memory, processor, etc.), the focus has been on optimizing time and resources, not on optimizing user consumption. Our work in this thesis has only been based on probabilistic topic models, but it would be convenient to address a more general problem to provide a 'sustainable' creation of learning models. In this sense, we would look for formats that reduce technological dependencies and that facilitate its use not only from a technical point of view, but also conceptually. Models should describe not only the information they provide, but also how it can be consumed, and detail the process that was followed to build it, as well as the resources that were used. In short, learning models should have standard formats, be self-contained, and offer semantically enabled interfaces.

Second, in this thesis we have not investigated the effect of model updates once a model has been learned/trained. This is a very common practice in other areas, for example fine tuning of language models, to improve an original model to solve a task. And this analysis adds a new dimension to what has been described above for distributing learning models, their path. As if they were pieces of a puzzle, models can be fitted together and as a result the path of the final model emerges. We do not refer to a processing pipeline, but to a conceptual path of data representation. If, for example, we have a fake news classifier built combining the BERT language model with a topic model trained on 20newsgroup and a Bayesian classifier, the final model would have to incorporate (not physically, but linked) the BERT model itself and the other models it uses (e.g. http://librairy.linkeddata.es/20news-model) to allow partial text representations from a single main access interface. Just as scientific articles cite works on which they are based, models should reference the models they use.

\subsubsection{Explainability of Text Similarity}

In Chapter 5, we have introduced a novel distance metric to compare texts from their topic distributions. The representativeness of topics to describe scientific articles was analyzed and results showed that abstracts were not sufficiently representative to describe, by means of topics, the content of a paper. Texts with greater vocabulary may emphasize key terms through repetition, favoring topic-based representation. Our work also highlights the difficulty in understanding the meaning of distances when there is no other element to justify their value. Figures 2.3 and 2.4, for example, showed how 
distances based on topic distributions between the same pairs of documents varies when the number of dimensions of the vector space changes (i.e. the number of topics). This highlights the difficulty of drawing conclusions from quantitative similarity measures. We elaborate on some key aspects below.

Our proposal to compare documents using only the most relevant topics, even establishing levels of importance, has proven its validity and would allow categorizing text similarity. We have not made progress in defining categories of similarity between documents, so as to facilitate their interpretation. Let us imagine that the similarity based on topics between two documents is 0.78 . Such a measure allows only relative conclusions (e.g. that they are more or less similar than other pairs of documents), but not absolute ones (e.g. that they are similar or different). The techniques presented in this thesis in Sections 5.2.1 and 6.1 can provide new mechanisms for comparing texts that facilitate their interpretation. It would be interesting to create a catalog of similarity levels and establish rules that transform current quantitative metrics into qualitative measures and evaluate it with humans.

But the categorization of similarity would not be enough to explain the relationship between documents. As we saw in Figures 6.5 and 6.6, the perception of similarity may vary with respect to the metric. Documents may share the main topics, but if they do not share any more topics in similar proportions and the representational model is sufficiently detailed (i.e. high number of topics), their similarity will be lower than if they share more less-relevant topics. It depends on the user consuming the similarity scores, for expert users the difference in these less relevant topics might be crucial, while for non experts it is just fine if we look at the salient topics. The level of detail of the model may represent the perception of a person when comparing documents. This raises an interesting discussion about the ability of probabilistic models, particularly those based on topics but applicable to others, to create contexts. And following this line, it would be necessary to analyze which characteristics of a corpus define the context it represents. A collection of scientific papers published at ISWC, K-CAP and ESWC conferences, for example, would be adequate to create a probabilistic model that contextualizes the semantic web knowledge, or on the contrary, papers from other disciplines may be needed to identify those aspects that can be differentiated. What kind of evaluations should be supported to measure the validity of these contextualization models?. 
Finally, the temporal aspect can be introduced and the evolution of topics could be also considered, either to relate texts described by means of topic distributions or to compare different versions of the same corpus. Some works measure the topic evolution from the communities that arise between the documents that mainly contain them (Gruhl et al., 2004, Kleinberg, 1999, Li et al., 2010), while others also consider the evolution of the communities themselves (Leydesdorff, 2012, Li et al., 2012, Nguyen et al. 2014, Prabhakaran et al., 2016; Zijun et al., 2018). Advancing in this line of research would enable comparisons, at corpus and document level, at a given instant in time.

\subsubsection{Complexity of Large-scale Comparisons}

The usefulness of topic distributions created by probabilistic models when exploring document collections at large-scale has been widely studied in the literature. In Chapter 6 we have introduced a new data structure to represent topic distributions based on topic hierarchies and hash codes. The approach has proven to obtain promising results and enable additional query restrictions based on the semantics offered by topics. Our comparison technique is based on the widely used nearest-neighbor approximations, which, however, has not been used as much in probabilistic topic models. The main reason is that they create binary representations based on Euclidean spaces, but the working spaces of probabilistic topic models are simplex. By creating hierarchical topic representations, we transform the space and can approximate the representation of documents with nearest-neighbor techniques.

However, this first step to represent texts by topic levels introduces new and interesting questions: what do these relevance levels mean?, how do they influence the comparison of documents?, are they model or domain independent?. In our experiments we found that the best performance was obtained with three levels of relevance, but no study has been done on the influence of of these hierarchies for solving other different tasks. We have started investigating on the influence of these levels for relating legal texts within the European Union. We also plan to extend the analysis in the academic domain to relate scientific texts. Our intention is to learn more about the meaning of these levels of relevance and to take advantage of it to organize documents at large-scale. It is also necessary to design evaluations that shed light on the inter- 
pretation that humans make of the relevance levels. The perception of similarity levels may require domain-dependent studies.

\subsubsection{Multilingual Representations}

In Chapter 7 we propose a concept-based representation of documents to create a shared representational space across different languages. The main contribution is that topics are created independently for each language, and are projected on concepts instead of words to relate them. The creation of unique representation spaces are widely adopted in the literature, although they usually rely on supervised methods. The main value of our proposal is that it does not need parallel or comparable corpus, or translations to create a single representation space. As expected, the performance in terms of accuracy is not as good as that of the supervised approach based on prior knowledge, and better in terms of coverage. And this makes us think that the word-based approach to abstracting the representation can be improved.
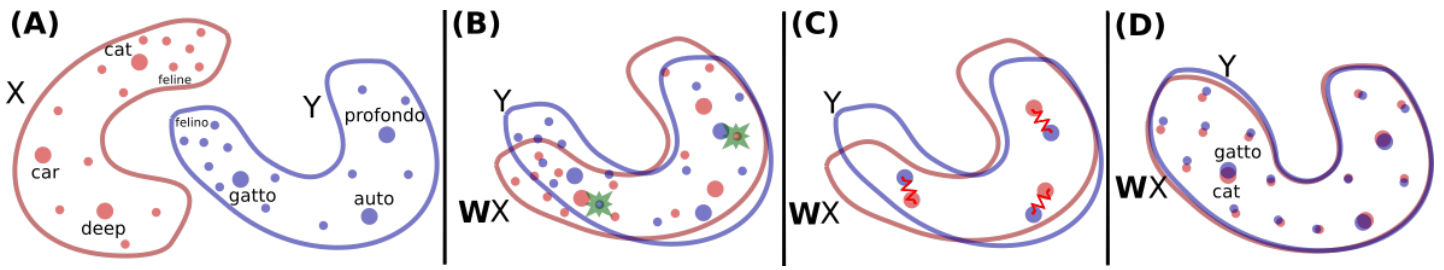

Figure 9.3: MUSE: Multilingual Unsupervised and Supervised Embeddings (Lample et al. 2018)

One of the main paradigms to relate multilingual texts is word alignment (Joulin et al., 2018; Lample et al., 2018). This approach learns word embeddings in different languages and maps them to a shared space through linear transformations (Figure 9.3). Unsupervised machine translation systems are created on these multilingual representations and mechanisms to create synthetic parallel corpora are even proposed Artetxe et al., 2019). But all these works are based on the same premise: there is a word-level correspondence between languages. However, this premise may not be true when relationships are established at the topic level. Our studies on legal texts annotated with cross-lingual EUROVOC categories revealed low performance when automatically classifying documents using probabilistic topics (Section 7.2.1). This makes us think that the premise of word correspondence, as the model is based on bags-of-words, may not 
be adequate to relate multilingual texts. The interpretation of a probabilistic topic, as a density distribution over the dictionary of words, may have to be evolved in order to move across languages. A topic is usually projected in a shared space as an aggregation of words or from an automatically generated label. With this in mind, it may be necessary to consider information units other than words, as language models are doing when working at the character level (Rami et al., 2019). 


\section{Bibliography}

Agerri, R., Bermudez, J., and Rigau, G. (2014). IXA pipeline: Efficient and Ready to Use Multilingual NLP tools. In Proceedings of the 9th Language Resources and Evaluation Conference (LREC), pages 26-31. 29

Aletras, N., Baldwin, T., Lau, J., and Stevenson, M. (2017). Evaluating topic representations for exploring document collections. Journal of the Association for Information Science and Technology, 68(1):154-167. 110

Ammar, W., Groeneveld, D., Bhagavatula, C., Beltagy, I., Crawford, M., Downey, D., Dunkelberger, J., Elgohary, A., Feldman, S., Ha, V., Kinney, R., Kohlmeier, S., Lo, K., Murray, T., Ooi, H., Peters, M., Power, J., Skjonsberg, S., Wang, L., Wilhelm, C., Yuan, Z., van Zuylen, M., and Etzioni, O. (2018). Construction of the Literature Graph in Semantic Scholar. In NAACL. 109

Andoni, A. and Indyk, P. (2006). Near-Optimal Hashing Algorithms for Approximate Nearest Neighbor in High Dimensions. In 2006 47th Annual IEEE Symposium on Foundations of Computer Science (FOCS'06), pages 459-468. IEEE. 33,102

Andoni, A., Indyk, P., Nguyen, H. L., and Razenshteyn, I. (2014). Beyond LocalitySensitive Hashing. In Proceedings of the Twenty-Fifth Annual ACM-SIAM Symposium on Discrete Algorithms, pages 1018-1028. 33, 102

Artetxe, M., Labaka, G., and Agirre, E. (2019). Bilingual lexicon induction through unsupervised machine translation. In Proceedings of the 57th Annual Meeting of the Association for Computational Linguistics, pages 5002-5007, Florence, Italy. Association for Computational Linguistics. 172 
Badenes-Olmedo, C., Chaves-Fraga, D., Poveda-Villalon, M., Iglesias-Molina, A., Calleja, P., Bernardos, S., Martin-Chozas, P., Fernandez-Izquierdo, A., AmadorDominguez, E., Espinoza-Arias, P., Pozo, L., Ruckhaus, E., Gonzalez-Guardia, E., Cedazo, R., Lopez-Centeno, B., and Corcho, O. (2020a). Drugs4covid: Drug-driven knowledge exploitation based on scientific publications. 154

Badenes-Olmedo, C., Redondo-García, J., and Corcho, O. (2017a). An Initial Analysis of Topic-based Similarity among Scientific Documents Based on their Rhetorical Discourse Parts. In Garijo, D., van Hage, W., Kauppinen, T.and Kuhn, T., and Zhao, J., editors, Proceedings of the First Workshop on Enabling Open Semantic Science co-located with 16th International Semantic Web Conference (ISWC), volume 1931 of CEUR Workshop Proceedings, pages 15-22. CEUR-WS.org. 47, 71

Badenes-Olmedo, C., Redondo-García, J., and Corcho, O. (2017b). Distributing Text Mining tasks with librAIry. In 17th ACM Symposium on Document Engineering (DocEng). 47, 51, 87

Badenes-Olmedo, C., Redondo-García, J., and Corcho, O. (2019a). Legal document retrieval across languages: topic hierarchies based on synsets. Proceedings of the 1st Workshop on Iberlegal co-located with 32nd International Conference on Legal Knowledge and Information Systems organized by the Foundation for Legal Knowledge Based Systems (JURIX). 47, 48, 50, 125, 164

Badenes-Olmedo, C., Redondo-García, J., and Corcho, O. (2019b). Scalable crosslingual document similarity through language-specific concept hierarchies. In Proceedings of the 10th International Conference on Knowledge Capture, pages 147-153. 150, 101, 125, 153, 164

Badenes-Olmedo, C., Redondo-García, J., and Corcho, O. (2020b). Large-Scale Semantic Exploration of Scientific Literature using Topic-based Hashing Algorithms. Semantic Web. xvii, 31, 32, 48

Badenes-Olmedo, C., Redondo-García, J. L., and Corcho, O. (2017c). Efficient Clustering from Distributions over Topics. In 9th International Conference on Knowledge Capture (K-CAP), page 8. 47, 71 
Bagga, A. and Baldwin, B. (1998). Algorithms for scoring coreference chains. In Proceedings of the 1st international conference on language resources and evaluation workshop on linguistics coreference, pages 563-566. 131

Bahmani, B., Moseley, B., Vattani, A., Kumar, R., and Vassilvitskii, S. (2012). Scalable K-Means ++. Proceedings of the VLDB Endowment (PVLDB), 5:622-633. 88

Basseville, M. (1989). Distance measures for signal processing and pattern recognition. Signal Process, 18(4):349-369. 25

Bizer, C., Heath, T., and Berners-Lee, T. (2009). Linked data-the story so far. International journal on Semantic Web and Information Systems, 5(3):1-22. 54

Blei, D. and Lafferty, J. D. (2007). A correlated topic model of Science. The Annals of Applied Statistics, 1(1):17-35. 25

Blei, D., Ng, A., and Jordan, M. (2003). Latent Dirichlet Allocation. Journal of Machine Learning Research, 3(4-5):993-1022. 18, 20, 74, 129

Bond, F. and Foster, R. (2013). Linking and Extending an Open Multilingual Wordnet. Proceedings of the 51st Annual Meeting of the Association for Computational Linguistics (ACL), pages 1352-1362. 125

Bond, F. and Paik, K. (2012). A survey of wordnets and their licenses. In Proceedings of the 6th Global WordNet Conference (GWC 2012), pages 64-71. 125

Boyd-Graber, J. and Blei, D. (2009). Multilingual topic models for unaligned text. In Proceedings of the Twenty-Fifth Conference on Uncertainty in Artificial Intelligence, UAI '09, page 75-82. AUAI Press. 35, 123

Boyd-Graber, J. and Resnik, P. (2010). Holistic Sentiment Analysis Across Languages: Multilingual Supervised Latent Dirichlet Allocation. Proceedings of the 2010 Conference on Empirical Methods in Natural Language Processing, pages 45-55. 25, 37, 123

Celikyilmaz, A., Hakkani-Tur, D., and Tur, G. (2010). LDA Based Similarity Modeling for Question Answering. In Proceedings of the NAACL HLT 2010 Workshop on Semantic Search, pages 1-9. 23 
Cha, S. (2007). Comprehensive Survey on Distance/Similarity Measures between Probability Density Functions. International Journal of Mathematical Models and Methods in Applied Sciences, 1(4):1-8. 110

Charikar, M. (2002). Similarity estimation techniques from rounding algorithms. In Proceedings of the thiry-fourth annual ACM symposium on Theory of computing STOC '02, page 380. ACM Press. 24

Chen, Y., Liu, Y., and Li, V. O. K. (2018). Zero-resource neural machine translation with multi-agent communication game. In Proceedings of the 32nd AAAI Conference on Artificial Intelligence, page 5086-5093. AAAI Press. 123

Cheng, X., Yan, X., Lan, Y., and Guo, J. (2014). BTM : Topic Modeling over Short Texts. IEEE Transactions on Knowledge and Data Engineering, 26(12):2928-2941. 19,129

Cohan, A. and Goharian, N. (2015). Scientific Article Summarization Using CitationContext and Article's Discourse Structure. In Conference on Empirical Methods in Natural Language Processing, pages 390-400. 73

Dabre, R., Chu, C., and Kunchukuttan, A. (2020). A survey of multilingual neural machine translation. ACM Computing Surveys (CSUR), 53:1-38. 123

Dagan, I., Lee, L., and Pereira, F. (1999). Similarity-Based Models of Word Cooccurrence Probabilities. Machine Learning, 34(1-3):43-69. 23,75

DasGupta, A. (2011). Probability for Statistics and Machine Learning. Springer Texts in Statistics. 25

Datar, M., Immorlica, N., Indyk, P., and Mirrokni, V. (2004). Locality-sensitive hashing scheme based on p-stable distributions. In Proceedings of the twentieth annual symposium on Computational geometry - SCG '04, page 253. ACM Press. 33,102

De Smet, W. and Moens, M. (2009). Cross-language linking of news stories on the web using interlingual topic modelling. In Proceedings of the 2nd ACM Workshop on Social Web Search and Mining, SWSM '09, page 57-64. Association for Computing Machinery. 35 
De Smet, W., Tang, J., and Moens, M. (2011). Knowledge Transfer across Multilingual Corpora via Latent Topics. In Advances in Knowledge Discovery and Data Mining, pages 549-560. 35

Deerwester, S., Dumais, S. T., Furnas, G. W., Landauer, T. K., and Harshman, R. (1990). Indexing by Latent Semantic Analysis. Journal of the American Society for Information Science, 41(6):391-407. 18, 20, 52

Dieng, A. B., Ruiz, F., and Blei, D. (2020). Topic modeling in embedding spaces. Transactions of the Association for Computational Linguistics, 8:439-453. 19

Divoli, A., Nakov, P., and Hearst, M. (2012). Do peers see more in a paper than its authors? Advances in Bioinformatics. 27, 72

Dong, F., O’Donoghue, D., Ersotelos, N., Wu, S., Saggion, H., Ronzano, F., Corcho, O., Hurley, D., Abgaz, Y., Zhang, J., Chaudhry, E., Yang, X., Wei, H., Deng, Z., Mahdian, B., and Careil, J. (2017). Dr.inventor, promoting scientific creativity by utilising web-based research objects. Impact, pages 40-44. 146

Dragoni, N., Giallorenzo, S., Lafuente, A., Mazzara, M., Montesi, F., Mustafin, R., and Safina, L. (2016). Microservices: yesterday, today, and tomorrow. CoRR, abs/1606.0:1-17. 60

Endres, D. and Schindelin, J. (2003). A new metric for probability distributions. IEEE Transactions on Information Theory, 49(7):1858-1860. 26

Ester, M., Kriegel, H., Sander, J., and X., X. (1996). A density-based algorithm for discovering clusters in large spatial databases with noise. In Proceedings of the Second International Conference on Knowledge Discovery and Data Mining, KDD'96, pages 226-231. AAAI Press. 89

Eurovoc (1995). Thesaurus EUROVOC - Volume 2: Subject-Oriented Version. Ed. 3/English Language. Annex to the index of the Official Journal of the EC. In Luxembourg, Office for Official Publications of the European Communities. 129

Fielding, R. and Taylor, R. (2002). Principled Design of the Modern Web Architecture. ACM Transactions on Internet Technology, 2(2):407-416. 59 
for Pharma, E. R. S. and Sciences, L. (2016). Harnessing the power of content Extracting value from scientific literature: the power of mining full-text articles for pathway analysis. Elsevier Inc. 72

Fukumasu, K., Eguchi, K., and Xing, E. P. (2012). Symmetric correspondence topic models for multilingual text analysis. In Proceedings of the 25th annual conference on advances in neural information processing systems (NIPS), pages 1295-1303. 34

Ganguly, D., Leveling, J., and Jones, G. (2012). Cross-Lingual Topical Relevance Models. In Proceedings of COLING 2012, pages 927-942. 35

Garcia-Silva, A. and Gómez-Pérez, J. (2021). Classifying scientific publications with bert - is self-attention a feature selection method? In Advances in Information Retrieval, pages 161-175, Cham. Springer International Publishing. 17

Gatti, C., Brooks, J., and Nurre, S. (2015). A Historical Analysis of the Field of OR/MS using Topic Models. CoRR, abs/1510.0. 19

Gliozzo, A. M., Pennacchiotti, M., and Pantel, P. (2007). The domain restriction hypothesis: Relating term similarity and semantic consistency. In Human Language Technologies 200\%: The Conference of the North American Chapter of the Association for Computational Linguistics; Proceedings of the Main Conference, pages 131-138. Association for Computational Linguistics. 131

Gómez-Carpintero, T. (2020). Analizador de lectura facil 4.0. Open Archive UPM http://oa.upm.es/63357/. 166

Greene, D. and Cross, J. (2016). Exploring the political agenda of the european parliament using a dynamic topic modeling approach. Political Analysis, 25(1):77-94. 19, 51

Greengrass, E. (2000). Information Retrieval: A Survey. University of Maryland. 12

Griffiths, T. and Steyvers, M. (2004). Finding scientific topics. Proceedings of the National Academy of Sciences of the United States of America, 101 Suppl:5228-35. 110 
Griffiths, T., Steyvers, M., and Tenenbaum, J. (2007). Topics in semantic representation. Psychological Review, 114(2):211-244. 3, 24

Gruhl, D., Guha, R., Liben-Nowell, D., and Tomkins, A. (2004). Information diffusion through blogspace. In Proceedings of the 13th International Conference on World Wide Web, WWW '04, page 491-501. Association for Computing Machinery. 171

Gutierrez, E., Shutova, E., Lichtenstein, P., Melo, G. d., and Gilardi, L. (2016). Detecting Cross-cultural Differences Using a Multilingual Topic Model. Transactions of the Association for Computational Linguistics, 4:47-60. 123

Halkidi, M., Batistakis, Y., and Vazirgiannis, M. (2001). On clustering validation techniques. Journal of Intelligent Information Systems, 17(2-3):107-145. 83

Hall, D., Jurafsky, D., and Manning, C. D. (2008). Studying the History of Ideas Using Topic Models. In Proceedings of the 2008 Conference on Empirical Methods in Natural Language Processing, Honolulu, October 2008, pages 363-371. 25

Hao, S., Boyd-Graber, J., and Paul, M. (2018). Lessons from the Bible on Modern Topics: Adapting Topic Model Evaluation to Multilingual and Low-Resource Settings. In Proceedings of the 2018 Conference of the North American Chapter of the Association for Computational Linguis- tics: Human Language Technologies, NAACL-HLT, pages 1090-1100. 37

Hao, S. and Paul, M. (2018). Learning multilingual topics from incomparable corpora. In Proceedings of the 27th International Conference on Computational Linguistics, pages 2595-2609. Association for Computational Linguistics. 35, 167

Harmon, D. K. (1996). Overview of the Third Text Retrieval Conference (TREC-3). DIANE Publishing Company. 16

He, J., Li, L., and Wu, X. (2017). A self-adaptive sliding window based topic model for non-uniform texts. In Proceedings - IEEE International Conference on Data Mining, ICDM, volume 2017-Novem, pages 147-156. 19,51

Hearst, M. a. and Hall, S. (1999). Untangling Text Data Mining. In the 37th Annual Meeting of the Association for Computational Linguistics, pages 1-13. 16 
Herranz, O. and Arenas-Garcia, J. (2019). Categorización de la oferta publica mediante tecnicas big data. e-archivo UC3M. 166

Hoffman, M. D., Blei, D. M., and Bach, F. (2010). Online learning for latent dirichlet allocation. In Proceedings of the 23rd International Conference on Neural Information Processing Systems - Volume 1, NIPS'10, page 856-864. Curran Associates Inc. 21

Hofmann, T. (2001). Unsupervised Learning by Probabilistic Latent Semantic Analysis. Machine Learning, 42(1-2):177-196. 18, 20

Hong, L. and Davison, B. (2010). Empirical study of topic modeling in Twitter. Proceedings of the First Workshop on Social Media Analytics - SOMA '10, pages 80-88. 76

Hsin-Min, L., Chih-Ping, W., and Fei-Yuan, C. (2016). Modeling healthcare data using multiple-channel latent Dirichlet allocation. Journal of Biomedical Informatics, 60:210-223. 19

Hu, Y., Zhai, K., Eidelman, V., and Boyd-Graber, J. (2014). Polylingual Tree-Based Topic Models for Translation Domain Adaptation. In Proceedings of the 52nd Annual Meeting of the Association for Computational Linguistics, pages 1166-1176. 129

Huang, L., Yang, Q., and Zheng, W. S. (2018). Online Hashing. IEEE Transactions on Neural Networks and Learning Systems, 29(6):2309-2322. 107

Indyk, P. and Motwani, R. (1998). Approximate nearest neighbors: Towards removing the curse of dimensionality. In Proceedings of the Thirtieth Annual ACM Symposium on Theory of Computing, STOC '98, page 604-613. Association for Computing Machinery. 33

Jagarlamudi, J. and Daumé, H. (2010). Extracting multilingual topics from unaligned comparable corpora. In Proceedings of the 32nd European Conference on Advances in Information Retrieval, ECIR'2010, page 444-456. Springer-Verlag. 35

Ji, J., Li, J., Yan, S., Tian, Q., and Zhang, B. (2013). Min-Max Hash for Jaccard Similarity. In 2013 IEEE 13th International Conference on Data Mining, pages 301-309. IEEE. 34, 102,126 
Johnson, R., Watkinson, A., and Mabe, M. (2018). The STM report: An overview of scientific and scholarly journal publishing. International Association of Scientific, Technical and Medical Publishers. 1

Joulin, A., Bojanowski, P., Mikolov, T., Jégou, H., and Grave, E. (2018). Loss in translation: Learning bilingual word mapping with a retrieval criterion. In Proceedings of the 2018 Conference on Empirical Methods in Natural Language Processing. 172

Kaliyar, R. K. (2020). A multi-layer bidirectional transformer encoder for pre-trained word embedding: A survey of bert. In 10th International Conference on Cloud Computing, Data Science Engineering (Confluence), pages 336-340. 124

Kandimalla, B., Rohatgi, S., Wu, J., and Giles, C. L. (2021). Large scale subject category classification of scholarly papers with deep attentive neural networks. Frontiers in Research Metrics and Analytics, 5:31. 17

Kenter, T. and Rijke, M. (2015). Short Text Similarity with Word Embeddings Categories and Subject Descriptors. Proceedings of 24 th ACM International Conference on Information and Knowledge Management, pages 1411-1420. 3

Kleinberg, J. M. (1999). Authoritative sources in a hyperlinked environment. J. ACM, 46(5):604-632. 171

Krstovski, K. and Smith, D. (2011). A Minimally Supervised Approach for Detecting and Ranking Document Translation Pairs. In Workshop on Statistical MT. 33

Krstovski, K., Smith, D., Wallach, H., and McGregor, A. (2013). Efficient NearestNeighbor Search in the Probability Simplex. In Proceedings of the 2013 Conference on the Theory of Information Retrieval - ICTIR '13, pages 101-108. ACM Press. 34 , 102,110

Kulis, B. and Grauman, K. (2012). Kernelized Locality-Sensitive Hashing. IEEE Transactions on Pattern Analysis and Machine Intelligence, 34(6):1092-1104. 33. 102

Kullback, S. (1968). Information theory and statistics. NewYork: Dover. 22 
Kullback, S. and Leibler, R. A. (1951). On information and sufficiency. The Annals of Mathematical Statistics, 22(1):79-86. 22

Lample, G., Conneau, A., Ranzato, M., Denoyer, L., and Jégou, H. (2018). Word translation without parallel data. In 6th International Conference on Learning Representations. OpenReview.net. xxi, 172

Le, Q. and Mikolov, T. (2014). Distributed representations of sentences and documents. In Proceedings of the 31st International Conference on Machine Learning, ICML'14, page II-1188-II-1196. JMLR.org. 17

Lee, J. H. (1995). Combining multiple evidence from different properties of weighting schemes. In Proceedings of the 18th Annual International ACM SIGIR Conference on Research and Development in Information Retrieval, SIGIR '95, page 180-188. Association for Computing Machinery. 16

Levy, O., Goldberg, Y., and Dagan, I. (2015). Improving distributional similarity with lessons learned from word embeddings. Transactions of the Association for Computational Linguistics, 3:211-225. 17

Leydesdorff, L. (2012). Statistics for the dynamic analysis of scientometric data: the evolution of the sciences in terms of trajectories and regimes. Scientometrics, 96:731741. 171

Li, D., He, B., Ding, Y., Tang, J., Sugimoto, C., Qin, Z., Yan, E., Li, J., and Dong, T. (2010). Community-based topic modeling for social tagging. In Proceedings of the 19th ACM International Conference on Information and Knowledge Management, CIKM '10, page 1565-1568. Association for Computing Machinery. 171

Li, P. and König, C. (2010). b-Bit minwise hashing. In Proceedings of the 19th international conference on World wide web - WWW'10, page 671. ACM Press. 34, 102, 126

Li, P., Owen, A., and Zhang, C. (2012). One Permutation Hashing. Advances in Neural Information Processing, 6(2):237-253. 34, 102, 126, 171

Lin, J. (1991). Divergence Measures Based on the Shannon Entropy. IEEE Transactions on Information Theory, 37(1):145-151. 23 
Lisena, P., Harrando, I., Kandakji, O., and Troncy, R. (2020). ToModAPI: A Topic Modeling API to Train, Use and Compare Topic Models. In 2nd International Workshop for Natural Language Processing Open Source Software (NLP-OSS). 29, 159

Litschko, R., Vulić, I., Ponzetto, S. P., and Glavaš, G. (2021). Evaluating multilingual text encoders for unsupervised cross-lingual retrieval. In Advances in Information Retrieval, pages 342-358, Cham. Springer International Publishing. 17

Liu, X., Duh, K., and Matsumoto, Y. (2015a). Multilingual Topic Models for Bilingual Dictionary Extraction. ACM Transactions on Asian \& Low-Resource Langanguage Information Processing, 14(3):1-22. 123

Liu, X., Zeng, J., Yang, X., Yan, J., and Yang, Q. (2015b). Scalable parallel em algorithms for latent dirichlet allocation in multi-core systems. In Proceedings of the 24th International Conference on World Wide Web, WWW'15, page 669-679. International World Wide Web Conferences Steering Committee. 52

López-Centeno, B., Badenes-Olmedo, C., Mataix-Sanjuan, A., McAllister, K., Bellón, J. M., Gibbons, S., Balsalobre, P., Pérez-Latorre, L., Benedí, J., Marzolini, C., Aranguren-Oyarzábal, A., Khoo, S., Calvo-Alcántara, M. J., and Berenguer, J. (2019). Polypharmacy and Drug-Drug Interactions in People Living With Human Immunodeficiency Virus in the Region of Madrid, Spain: A Population-Based Study. Clinical Infectious Diseases, pages 352-362. खx, 149, 151, 159, 166

Lozano, B., Badenes-Olmedo, C., and Corcho, O. (2020). The Influence of Text Length for Probabilistic Topic Modelsand their HierarchicalRepresentation. Open Archive UPM - http://oa.upm.es/63753/. 134

Ma, T. and Nasukawa, T. (2017). Inverted Bilingual Topic Models for Lexicon Extraction from Non-parallel Data. In Proceedings of the Twenty-Sixth International Joint Conference on Artificial Intelligence, pages 4075-4081. 123

Manning, C., Surdeanu, M., Bauer, J., Finkel, J., Bethard, S., and McClosky, D. (2014). The Stanford CoreNLP Natural Language Processing Toolkit. In Proceedings of 52nd Annual Meeting of the Association for Computational Linguistics: System Demonstrations. 29 
Manning, C. D., Raghavan, P., and Schutze, H. (2008). Introduction to Information Retrieval. Cambridge University Press. 11

Mao, X., Feng, B., Hao, Y., Nie, L., Huang, H., and Wen, G. (2017). S2JSD-LSH: A Locality-Sensitive Hashing Schema for Probability Distributions. In AAAI. 34, 102 , 110, 161

Mikolov, T., Sutskever, I., Chen, K., Corrado, G., and Dean, J. (2013). Distributed Representations of Words and Phrases and their Compositionality. Proceedings of the 26th International Conference on Neural Information Processing Systems, 2:31113119. 17

Miller, G. A. (1995). WordNet: A Lexical Database for English. Communications of the ACM, 38(11):39-41. 124, 164

Mimno, D., Wallach, H., Naradowsky, J., Smith, D., and McCallum, A. (2009). Polylingual topic models. In Proceedings of the 2009 Conference on Empirical Methods in Natural Language Processing, EMNLP '09, pages 880-889. Association for Computational Linguistics. 34,35

Mimno, D., Wallach, H., Talley, E., Leenders, M., and McCallum, A. (2011). Optimizing Semantic Coherence in Topic Models. In Proceedings of the 2011 Conference on Empirical Methods in Natural Language Processing, pages 262-272. Association for Computational Linguistics. 131

Moritz, M. and Büchler, M. (2017). Ambiguity in Semantically Related Word Substitutions: an Investigation in Historical Bible Translations. In Proceedings of the NoDaLiDa 2017 Workshop on Processing Historical Language, pages 18-23. 37

Nakov, P. and Ng, H. T. (2009). Improved statistical machine translation for resourcepoor languages using related resource-rich languages. In Proceedings of the 2009 Conference on Empirical Methods in Natural Language Processing, pages 1358-1367. Association for Computational Linguistics. 123

Neubig, G. and $\mathrm{Hu}$, J. (2018). Rapid adaptation of neural machine translation to new languages. In Proceedings of the 2018 Conference on Empirical Methods in Natural Language Processing, pages 875-880. Association for Computational Linguistics. 123 
Newman, D., Lau, J., Grieser, K., and Baldwin, T. (2010). Automatic Evaluation of Topic Coherence. In Human Language Technologies: The 2010 Annual Conference of the North $\{A\}$ merican Chapter of the Association for Computational Linguistics, pages 100-108. 131

Nguyen, N. P., Dinh, T., Shen, Y., and Thai, M. (2014). Dynamic social community detection and its applications. PLOS ONE, 9. 171

Ni, X., Sun, J., Hu, J., and Chen, Z. (2009). Mining multilingual topics from wikipedia. In Proceedings of the 18th International Conference on World Wide Web, WWW '09, page 1155-1156. Association for Computing Machinery. 34,123

Ni, X., Sun, J., Hu, J., and Chen, Z. (2011). Cross Lingual Text Classification by Mining Multilingual Topics from Wikipedia. In Proceedings of the Fourth ACM International Conference on Web Search and Data Mining, pages 375-384. 35, 123

Nzali, T., Donald, M., Bringay, S., Lavergne, C., Mollevi, C., and Opitz, T. (2017). What Patients Can Tell Us: Topic Analysis for Social Media on Breast Cancer. JMIR medical informatics, 5(3):e23. 19, 51

O'Neill, J., Robin, C., O'Brien, L., and Buitelaar, P. (2017). An analysis of topic modelling for legislative texts. CEUR Workshop Proceedings, 2143. 19, 51

Pan, S. J. and Yang, Q. (2010). A survey on transfer learning. IEEE Transactions on Knowledge and Data Engineering, 22(10):1345-1359. 124

Pennington, J., Socher, R., and Manning, C. (2014). GloVe: Global vectors for word representation. In Proceedings of the 2014 Conference on Empirical Methods in Natural Language Processing (EMNLP), pages 1532-1543. Association for Computational Linguistics. 17

Piccardi, T. and West, R. (2020). Crosslingual Topic Modeling with WikiPDA. arXiv e-prints. 35,163

Platt, J., Toutanova, K., and Yih, W. (2010). Translingual Document Representations from Discriminative Projections. In Proceedings of the 2010 Conference on Empirical Methods in Natural Language Processing, EMNLP '10, pages 251-261. Association for Computational Linguistics. 35 
Porter, M. F. (1997). An Algorithm for Suffix Stripping, page 313-316. Morgan Kaufmann Publishers Inc. 14

Prabhakaran, V., Hamilton, W., McFarland, D., and Jurafsky, D. (2016). Predicting the rise and fall of scientific topics from trends in their rhetorical framing. In Proceedings of the 54 th Annual Meeting of the Association for Computational Linguistics (Volume 1: Long Papers), pages 1170-1180, Berlin, Germany. Association for Computational Linguistics. 171

Ramachandran, P. and Vimala, D. (2014). Organization of a Research Paper: The IMRAD Format. Springer. 77

Ramage, D., Hall, D., Nallapati, R., and Manning, C. D. (2009). Labeled LDA: A supervised topic model for credit attribution in multi-labeled corpora. In Proceedings of the 2009 Conference on Empirical Methods in Natural Language Processing (EMNLP'09), pages 248-256. 130, 164

Rami, A., Dokook, C., Noah, C., Mandy, G., and Llion, J. (2019). Character-level language modeling with deeper self-attention. Proceedings of the AAAI Conference on Artificial Intelligence, 33(01):3159-3166. 173

Rao, C. R. (1982). Diversity: Its Measurement, Decomposition, Apportionment and Analysis. Sankhyā: The Indian Journal of Statistics, Series A, 44(1):1-22. 23

Ronzano, F. and Saggion, H. (2015). Dr. Inventor Framework: Extracting Structured Information from Scientific Publications. In Discovery Science: 18th International Conference, pages 209-220. 73, 147

Rus, V., Niraula, N., and Banjade, R. (2013). Similarity Measures Based on Latent Dirichlet Allocation. In Computational Linguistics and Intelligent Text Processing, volume 7816, pages 459-470. Springer. 23, 25, 75

Salatino, A., Osborne, F., Thanapalasingam, T., and Motta, E. (2019). The cso classifier: Ontology-driven detection of research topics in scholarly articles. In Digital Libraries for Open Knowledge, pages 296-311, Cham. Springer International Publishing. 17 
Salton, G. and McGill, M. J. (1983). Introduction to Modern Information Retrieval. McGraw-Hill, Inc. 12, 16

Samy, D., Perez-Fernandez, D., and Arenas-Garcia, J. (2019). Landscaping language technologies using topic modeling and graph analysis: Overview of the spanish contribution. Procesamiento del Lenguaje Natural, 63(0):129-136. 149, 159,166

Saracevic, T. (1995). Evaluation of evaluation in information retrieval. In Proceedings of the 18th Annual International ACM SIGIR Conference on Research and Development in Information Retrieval, SIGIR '95, page 138-146. Association for Computing Machinery. 13

Schofield, A., Magnusson, M., and Mimno, D. (2017). Pulling out the stops: Rethinking stopword removal for topic models. In Proceedings of the 15th Conference of the European Chapter of the Association for Computational Linguistics: Volume 2, Short Papers, pages 432-436, Valencia, Spain. Association for Computational Linguistics. 128

Shi, B., Lam, W., Bing, L., and Xu, Y. (2016). Detecting common discussion topics across culture from news reader comments. In Proceedings of the 54 th Annual Meeting of the Association for Computational Linguistics (Volume 1: Long Papers), pages 676-685. Association for Computational Linguistics. 35

Simone T. (2010). The Structure of Scientific Articles - Applications to Citation Indexing and Summarization. In CSLI Studies in Computational Linguistics. 72

Soylu, A., Corcho, O., Elvesæter, B., Badenes-Olmedo, C., Yedro, F., Kovacic, M., Posinkovic, M., Makgill, I., Taggart, C., Simperl, E., Lech, T., and Roman, D. (2020). Enhancing public procurement in the european union through constructing and exploiting an integrated knowledge graph. In Proceedings of the 19th International Semantic Web Conference (ISWC 2020). 152

Steinberger, R., Ebrahim, M., Poulis, A., Carrasco-Benitez, M., Schlüter, P., Przybyszewski, M., and Gilbro, S. (2014). An overview of the European Union's highly multilingual parallel corpora. Language Resources and Evaluation, 48(4):679-707. 123,135 
Steinberger, R., Pouliquen, B., Widiger, A., Ignat, C., Erjavec, T., Tufis, D., and Varga, D. (2006). The JRC-Acquis: A multilingual aligned parallel corpus with 20+ languages. In Proceedings of the 5th International Conference on Language Resources and Evaluation (LREC'2006), volume 4, pages 2142-2147. 129

Steyvers, M. and Griffiths, T. (2006). Probabilistic topic models. 87

Terasawa, K. and Tanaka, Y. (2007). Spherical LSH for Approximate Nearest Neighbor Search on Unit Hypersphere. In Algorithms and Data Structures, pages 27-38. 33, 102

Teufel, S., Siddharthan, A., and Batchelor, C. (2009). Towards discipline-independent Argumentative Zoning: Evidence from chemistry and computational linguistics. Conference on Empirical Methods in Natural Language Processing, pages 1493-1502. 73

Towne, W., Rosé, C., and Herbsleb, J. (2016). Measuring similarity similarly: Lda and human perception. ACM Transactions on Intelligent Systems and Technology, $8(1): 1-25.85,110$

Turchi, S., Ciofi, L., Paganelli, F., Pirri, F., and Giuli, D. (2012). Designing EPCIS through Linked Data and REST principles. Software, Telecommunications and Computer Networks $(\{$ SoftCOM $)\}$, 2012 20th International Conference on, pages 1-6. 54

Vijayanarasimhan, S., Jain, P., and Grauman, K. (2014). Hashing Hyperplane Queries to Near Points with Applications to Large-Scale Active Learning. IEEE Transactions on Pattern Analysis and Machine Intelligence, 36(2):276-288. 34, 102

Vulić, I., De Smet, W., Tang, J., and Moens, M. (2015). Probabilistic topic modeling in multilingual settings: An overview of its methodology and applications. Information Processing and Management, 51(1):111-147. 35, 123

Vulić, I. and Moens, M. (2012). Detecting Highly Confident Word Translations from Comparable Corpora Without Any Prior Knowledge. In Proceedings of the 13th Conference of the European Chapter of the Association for Computational Linguistics, pages 449-459. 35 
Vulić, I. and Moens, M. (2013). A Unified Framework for Monolingual and CrossLingual Relevance Modeling Based on Probabilistic Topic Models. In Advances in Information Retrieval, pages 98-109. 35

Wang, J., Liu, W., Kumar, S., and Chang, S. (2016). Learning to Hash for Indexing Big Data-A Survey. Proceedings of the IEEE, 104(1):34-57. 33, 102

Wang, L., Lo, K., Chandrasekhar, Y., Reas, R., Yang, J., Eide, D., Funk, K., Kinney, R., Liu, Z., and Merrill, W. (2020). CORD-19: The Covid-19 Open Research Dataset. arXiv preprint arXiv:2004.10706. 154

Wei, W. and Guo, C. (2019). A text semantic topic discovery method based on the conditional co-occurrence degree. Neurocomputing, 368:11-24. 17

Westergaard, D., Stærfeldt, H., Tønsberg, C., Jensen, L., and Brunak, S. (2017). Text mining of 15 million full-text scientific articles. bioRxiv. 27, 72, 159

Yang, W., Boyd-Graber, J., and Resnik, P. (2019). A multilingual topic model for learning weighted topic links across corpora with low comparability. In Proceedings of the 2019 Conference on Empirical Methods in Natural Language Processing and the 9th International Joint Conference on Natural Language Processing (EMNLPIJCNLP), pages 1243-1248. 35, 163

Yuan, M., Van Durme, B., and Ying, J. L. (2018). Multilingual anchoring: Interactive topic modeling and alignment across languages. In Advances in Neural Information Processing Systems, volume 31, pages 8653-8663. Curran Associates, Inc. 35, 163

Zhang, D., Mei, Q., and Zhai, C. (2010). Cross-lingual latent topic extraction. In Proceedings of the 48th Annual Meeting of the Association for Computational Linguistics, pages 1128-1137. Association for Computational Linguistics. 35

Zhang, T., Liu, K., and Zhao, J. (2013). Cross lingual entity linking with bilingual topic model. In Proceedings of the Twenty-Third International Joint Conference on Artificial Intelligence, IJCAI '13, page 2218-2224. AAAI Press. 34

Zhao, W., Jégou, H., and Gravier, G. (2013). Sim-min-hash. In Proceedings of the 21st ACM international conference on Multimedia - MM '13, pages 577-580. 34, 102, 126 
Zhen, Y., Gao, Y., Yeung, D., Zha, H., and Li, X. (2016). Spectral Multimodal Hashing and Its Application to Multimedia Retrieval. IEEE Transactions on Cybernetics, 46(1):27-38. 33,161

Zhu, Z., Li, M., Chen, L., and Yang, Z. (2013). Building Comparable Corpora Based on Bilingual \{LDA\} Model. In Proceedings of the 51st Annual Meeting of the Association for Computational Linguistics (Volume 2: Short Papers), pages 278-282. 35

Zijun, Y., Yifan, S., Weicong, D., Nikhil, R., and Hui, X. (2018). Dynamic word embeddings for evolving semantic discovery. In Proceedings of the Eleventh ACM International Conference on Web Search and Data Mining, WSDM '18, page 673-681, New York, NY, USA. Association for Computing Machinery. 171 